\title{
Gallai-Ramsey numbers for graphs and their generalizations
}

\section{Xihe Li}

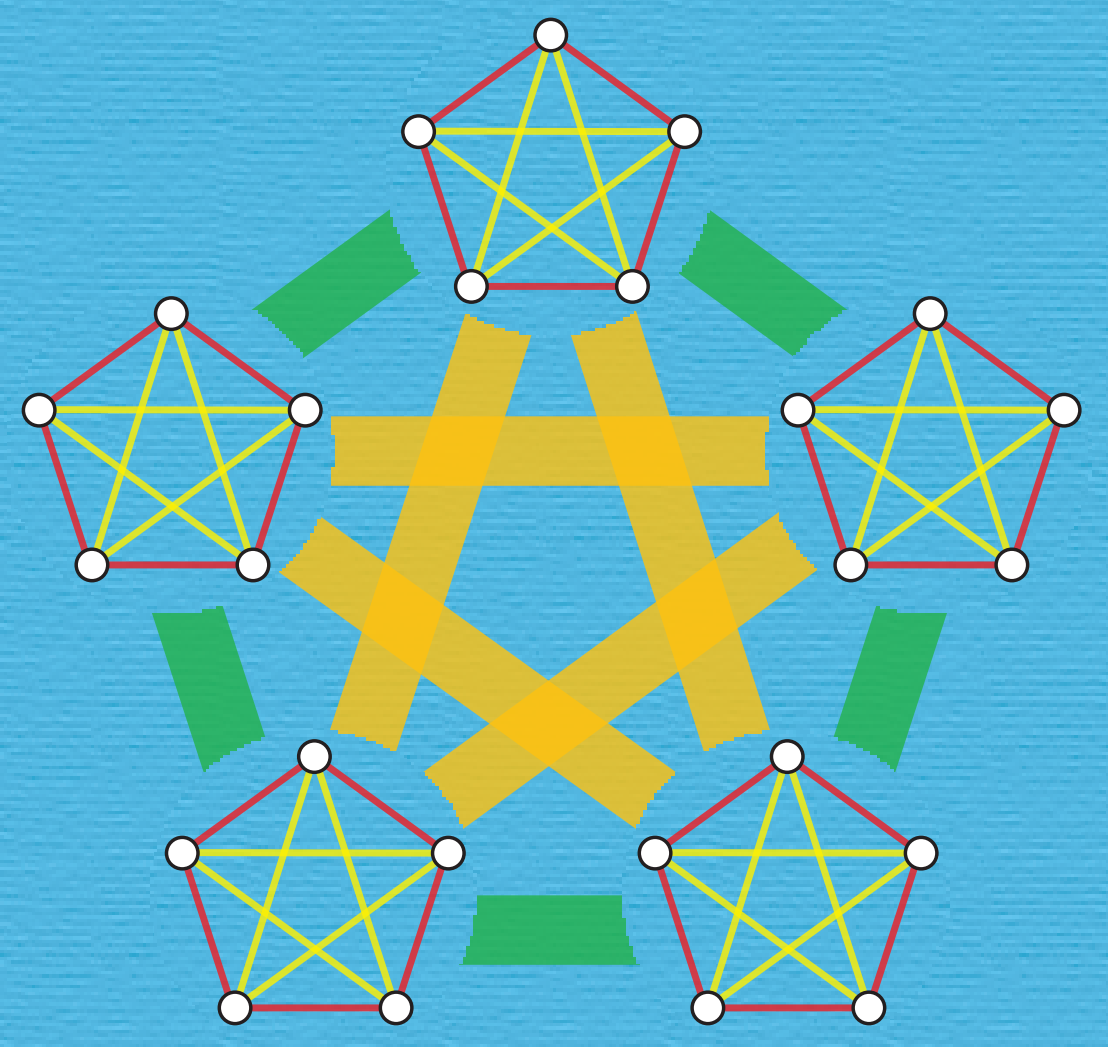

UNIVERSITY OF TWENTE. 

GALLAI-RAMSEY NUMBERS FOR GRAPHS AND THEIR GENERALIZATIONS

Xihe Li 



\section{GALLAI-RAMSEY NUMBERS FOR GRAPHS AND THEIR GENERALIZATIONS}

\section{DISSERTATION}

to obtain

the degree of doctor at the University of Twente, on the authority of the rector magnificus, prof. dr. ir. A. Veldkamp, on account of the decision of the Doctorate Board, to be publicly defended on Thursday the $21^{\text {st }}$ of October 2021 at 12.45 hours

by

\section{Xihe Li}

born on the $10^{\text {th }}$ of October 1995

in Dezhou, Shandong, China 
This dissertation has been approved by the supervisors:

prof. dr. ir. H.J. Broersma and prof. dr. L. Wang

The research reported in this thesis has been carried out within the framework of the MEMORANDUM OF AGREEMENT FOR A DOUBLE DOCTORATE DEGREE BETWEEN NORTHWESTERN POLYTECHNICAL UNIVERSITY, PEOPLE'S REPUBLIC OF CHINA AND THE UNIVERSITY OF TWENTE, THE NETHERLANDS.

DSI Ph.D. Thesis Series No. 21-010

UNIVERSITY $\mid$ DIGITAL SOCIETY Digital Society Institute

OF TWENTE. INSTITUTE P.O. Box 217, 7500 AE Enschede, The Netherlands.

ISBN: 978-90-365-5248-6

ISSN: 2589-7721 (DSI Ph.D. thesis Series No. 21-010)

DOI: $10.3990 / 1.9789036552486$

Available online at https://doi.org/10.3990/1.9789036552486

Typeset with $\mathrm{ET}_{\mathrm{E}} \mathrm{X}$

Printed by Ipskamp Printing, Enschede

Cover design by Xihe Li

(c) 2021 Xihe Li, The Netherlands. All rights reserved. No parts of this thesis may be reproduced, stored in a retrieval system or transmitted in any form or by any means without permission of the author. Alle rechten voorbehouden. Niets uit deze uitgave mag worden vermenigvuldigd, in enige vorm of op enige wijze, zonder voorafgaande schriftelijke toestemming van de auteur. 


\section{Graduation Committee}

Chairman/secretary:

Prof. dr. J.N. Kok

University of Twente

Supervisors:

Prof. dr. ir. H.J. Broersma University of Twente

Prof. dr. L. Wang Northwestern Polytechnical University

Members:

Prof. dr. J.L. Hurink University of Twente

Prof. dr. M.J. Uetz University of Twente

Prof. dr. M.A. Axenovich Karlsruher Institut für Technologie

Prof. dr. X. Li Nankai University

Prof. dr. I. Schiermeyer Technische Universität Bergakademie Freiberg 



\section{Preface}

This thesis contains research results on Gallai-Ramsey theory and its generalizations, which were obtained by the author with collaborators between September 2017 and February 2021. Apart from an introductory chapter (Chapter 1), the reader will find six closely related technical chapters (Chapters 2-7), which are mainly based on the research results that the author obtained when he was working as a PhD student at Northwestern Polytechnical University, Xi'an and the University of Twente.

Chapters 2 and 3 focus on determining the exact values of Gallai-Ramsey numbers for several graphs. The other chapters mainly focus on studying various generalizations or variants of Gallai-Ramsey theory. In Chapter 4, we consider two extremal problems related to Gallai-colorings. In Chapter 5, we study the Erdős-Gyárfás function with respect to Gallai-colorings. In Chapter 6, we present a forbidden rainbow subgraph condition for an edge-colored graph to have a highly-connected monochromatic subgraph. In Chapter 7, we deal with the rainbow Erdős-Rothschild problem with respect to 3-term arithmetic progressions.

\section{Papers underlying this thesis}

[1] Gallai-Ramsey numbers for a class of graphs with five vertices, Graphs and Combinatorics 36 (2020), 1603-1618 (with L. Wang). (Chapter 2)

[2] Extremal problems and results related to Gallai-colorings, Discrete Mathematics 344 (2021), 112567 (with H.J. Broersma and L. Wang). (Chapters 3 and 4$)$ 
[3] The Erdős-Gyárfás function with respect to Gallai-colorings, submitted (with H.J. Broersma and L. Wang).

(Chapter 5)

[4] Forbidden rainbow subgraphs that force large monochromatic or multicolored $k$-connected subgraphs, Discrete Applied Mathematics 285 (2020), 18-27 (with L. Wang).

(Chapter 6)

[5] Integer colorings with no rainbow 3-term arithmetic progression, submitted (with H.J. Broersma and L. Wang).

(Chapter 7)

\section{Other recent joint papers by the author}

[1] Complete graphs and complete bipartite graphs without rainbow path, Discrete Mathematics 342 (2019), 2116-2126 (with L. Wang and X. Liu).

[2] Monochromatic stars in rainbow $K_{3}$-free and $S_{3}^{+}$-free colorings, Discrete Mathematics 343 (2020), 112131 (with L. Wang).

[3] Gallai-Ramsey numbers for rainbow $S_{3}^{+}$and monochromatic paths, Discussiones Mathematicae Graph Theory (2019), in press (with L. Wang). 


\section{Contents}

Preface vii

1 Introduction 1

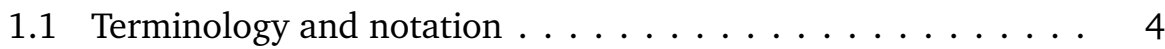

1.2 Ramsey theory . . . . . . . . . . . . . . . . 7

1.3 Generalizations of Ramsey theory . . . . . . . . . . . . . . . . . . . . 11

1.3.1 Gallai-Ramsey theory . . . . . . . . . . . . . 11

1.3.2 The Erdős-Gyárfás function $\ldots \ldots \ldots \ldots \ldots$

1.4 Outline of the main results of this thesis $\ldots \ldots \ldots \ldots 14$

2 Gallai-Ramsey numbers for a class of graphs with five vertices 17

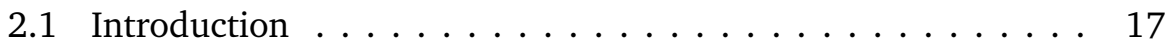

2.2 Gallai-Ramsey numbers for $F_{9}$ and $F_{10} \ldots \ldots \ldots \ldots$

2.3 (Gallai-)Ramsey numbers for $F_{2, n} \ldots \ldots \ldots \ldots \ldots$

2.4 Gallai-Ramsey numbers for $F_{12}$ and $F_{13} \ldots \ldots \ldots \ldots$

3 The Gallai-Ramsey number for $K_{4}+e \quad 35$

3.1 Introduction $\ldots \ldots \ldots \ldots \ldots \ldots \ldots \ldots \ldots \ldots \ldots$

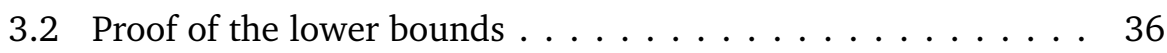

3.3 Proof of the upper bounds . . . . . . . . . . . 37

3.4 A short survey on known Gallai-Ramsey numbers . . . . . . . 43

4 Two extremal problems and results related to Gallai-colorings 51

4.1 Introduction $\ldots \ldots \ldots \ldots \ldots \ldots \ldots \ldots \ldots \ldots$

4.2 Preliminaries . . . . . . . . . . . . . . 54 
4.3 Results related to the number of edges . . . . . . . . . 55

4.4 The Gallai-Ramsey multiplicity problem . . . . . . . . . . . 61

4.5 Concluding remarks . . . . . . . . . . . . . . 64

5 The Erdős-Gyárfás function with respect to Gallai-colorings 67

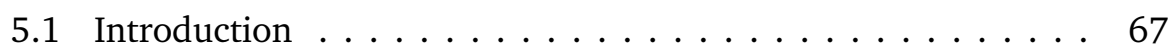

5.2 Preliminaries . . . . . . . . . . . . . . 71

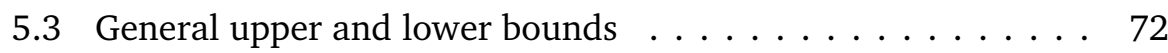

5.4 Proofs of Theorems 5.7 and $5.8 \ldots \ldots \ldots \ldots \ldots$

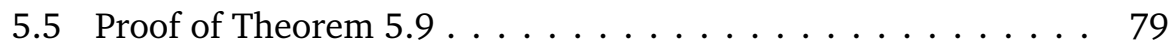

5.6 Proofs of Theorems 5.10 and $5.11 \ldots \ldots \ldots \ldots$

5.7 Proof of Theorem $5.12 \ldots \ldots \ldots \ldots$. . . . . . 87

5.8 Two results for $K_{6} \ldots \ldots \ldots \ldots \ldots \ldots \ldots \ldots$

5.9 Concluding remarks $\ldots \ldots \ldots \ldots \ldots$

6 Forbidden rainbow subgraphs that force large monochromatic or 2-colored $k$-connected subgraphs 101

6.1 Introduction . . . . . . . . . . . . . . . . . 102

6.2 A characterization of the set $\mathscr{H} \ldots \ldots \ldots \ldots$

6.3 Proof of Theorem $6.4 \ldots \ldots \ldots \ldots 112$

6.4 A characterization of the set $\mathscr{B} \ldots \ldots \ldots \ldots \ldots$

6.5 Large $k$-connected 2-colored subgraphs in every Gallai-3-coloring120

6.6 Concluding remarks . . . . . . . . . . . . . . 125

7 Integer colorings with no rainbow 3-term arithmetic progression 127

7.1 Introduction $\ldots \ldots \ldots \ldots \ldots \ldots \ldots \ldots \ldots \ldots \ldots \ldots$

7.2 Additional notation and preliminaries . . . . . . . 131

7.3 Colorings of integer sets with no rainbow 3-AP . . . . . . . 134

7.4 Colorings of $\mathbb{Z}_{n}$ with no rainbow 3-AP . . . . . . . . 140

$\begin{array}{ll}\text { Summary } & 145\end{array}$

Samenvatting (Summary in Dutch) 149

$\begin{array}{ll}\text { Bibliography } & 153\end{array}$ 
Acknowledgements

About the Author 



\section{Chapter 1}

\section{Introduction}

At the beginning of this chapter, we first present some intuitive background to the theme of this thesis, before introducing the formal terminology and notation in Section 1.1. With this part in mind, the reader will hopefully understand the content of this thesis more easily.

We begin by recalling the following "Party Problem" which was posed in The American Mathematical Monthly in 1958 [24]:

"Prove that at a gathering of any six people, some three of them are either mutual acquaintances (each of these three knows the other two people) or complete strangers to each other (each of these three knows neither of the other two people)."

In order to analyze and solve the above problem, we can model the relationships (the acquainted pairs) by means of a diagram, as follows. We represent the six people by six distinct and non-overlapping points $v_{1}, v_{2}, \ldots, v_{6}$, and we join any two points by a line segment or curve (avoiding the other four points) whenever the two corresponding people know each other. Then the above problem can be reformulated as follows. Prove that every such diagram on six points either contains three points that are all pairwise joined by a line segment or curve, or three points with no line segment or curve joining any two of them.

To prove this statement, we can start by looking at the point $v_{1}$. By the Pigeonhole Principle, there exist three points in $\left\{v_{2}, v_{3}, \ldots, v_{6}\right\}$, say $v_{2}, v_{3}$ and 
$v_{4}$ (possibly after permuting the labels), such that either all of them are joined to $v_{1}$ by a line segment or curve, or none of them is joined to $v_{1}$ by a line segment or curve. In the former case, we are clearly done if at least one of the pairs in $\left\{v_{2}, v_{3}, v_{4}\right\}$ is joined by a line segment or curve. But we are also done if none of the pairs in $\left\{v_{2}, v_{3}, v_{4}\right\}$ is joined by a line segment or curve. In the latter case, we can find three required points in a similar way, by reversing the role of the absence and presence of line segments or curves between pairs. We leave this as an exercise to the reader.

In graph theoretical language, the above diagram, and its points and line segments or curves are usually referred to as a (drawing of a) graph, and its vertices and edges, respectively. We will define these concepts properly and formally in the next section.

Graphs form a powerful tool in solving many real-world problems. In addition, graph theory itself has developed into a mature independent mathematical branch, which has wide applications in combinatorics, geometry, number theory, ergodic theory, measure theory, computer science, chemistry, physics, biology, sociology, and so on.

In this thesis, the main focus lies on extending existing theory related to the above problem. Historically, the main effort of graph theorists has been to prove similar results for larger groups of people, i.e., to determine the smallest number of people guaranteeing that there always exists a subgroup of acquaintances or non-acquaintances of a certain size. This resulted in what nowadays is referred to as Ramsey theory. This theory and its intriguing open problems will be introduced in Section 1.2.

Here we continue our layman's introduction with a second problem which generalizes the above Party Problem. This variant of the Party Problem will reveal that the existence or non-existence of acquaintanceships (or edges in the corresponding graph) is not always sufficient to model more complicated practical situations. Consider the following generalization of the Party Problem.

Suppose that we distinguish between three instead of two possible relationships between two people, say that they could be either friends, enemies or strangers. How many members should a group of people have in order to 
guarantee that there exist three members who are mutually either all friends, all enemies, or all strangers?

In this case, since there are three different relationships to consider, the above approach with the diagram and the corresponding graph is not suitable for analyzing or solving the problem. Here the known concept of an edgecolored graph comes in handy. As before, each member of the group is represented by a vertex in the graph. We choose three distinct colors to represent each of the three types of relationship. We assign one of these colors to each of the edges of the graph, representing the type of relationship between the corresponding pair of members in the group. So we consider a complete graph (i.e., in which each pair of vertices is joined by an edge) whose edges are assigned one of the three distinct colors. Now the problem can be formulated as the following graph problem. What is the smallest number of vertices in a complete graph guaranteeing that in any edge-coloring of this graph using three colors, there exists a complete subgraph on three vertices whose edges all have the same color. We remark that the answer to this question is 17, and that a proof can be found in [88]. Interestingly, it is rather easy to show that 17 is sufficient by using the solution to the Party Problem and similar arguments as above to start the reasoning, but it is more difficult to present an example showing that 16 is not always sufficient.

In the research on edge-colored graphs, one can distinguish two classes of problems. In the first class the problem is to determine the minimum number of colors needed to color the edges of a graph in such a way that the resulting edge-colored graph satisfies certain requirements. A typical example belonging to this first class was the (edge-coloring equivalent of the) Four-Color Conjecture (nowadays a well-known and celebrated theorem), the study of which has greatly promoted the development of graph theory. In the second class of problems the purpose is to study the existence of subgraphs with certain specific edge-colorings in a larger graph whose edges have already been colored. Typical examples of such problems can be found in the area of Ramsey theory, which is the main theme of this thesis. After providing some basic terminology and notation in Section 1.1, we will introduce Ramsey theory and its generalizations in Sections 1.2 and 1.3. In the final section of this chapter, we will provide an overview of our main contributions to the 
field. In the subsequent chapters, the reader will find the exact statements and proofs of our contributions, as well as more details related to the results included in this thesis.

\subsection{Terminology and notation}

In this section, we introduce some basic terminology and notation. All graphs considered in this thesis are finite undirected graphs without loops or multiple edges, unless otherwise indicated. We use [22] for terminology and notation not defined here.

Let $G=(V(G), E(G))$ be a graph, where $V(G)$ is the set of vertices of $G$ and $E(G)$ is the set of edges of $G$. The order and size of $G$ are defined to be the cardinality of $V(G)$ and $E(G)$, respectively. For a vertex $v \in V(G)$, let $N_{G}(v)$ be the set of neighbors of $v$ in $G$, i.e., the set of all vertices adjacent to $v$. The degree of a vertex $v$ in $G$, denoted by $d_{G}(v)$, is the number of edges incident with $v$ in $G$. Note that $d_{G}(v)=\left|N_{G}(v)\right|$ for a (simple) graph $G$. We use $\Delta(G)=\max \left\{d_{G}(v): v \in V(G)\right\}$ and $\delta(G)=\min \left\{d_{G}(v): v \in V(G)\right\}$ to denote the maximum degree and minimum degree of $G$, respectively. If two distinct vertices $u$ and $v$ are adjacent in the graph $G$, then we denote the edge $e$ of $G$ joining $u$ and $v$ simply as $u v$, and we say that $u$ and $v$ are the end-vertices of $e$. If an edge of $G$ has one end-vertex with degree 1 in $G$, we sometimes call it a pendant edge.

We next introduce some special families of graphs. We refer to Figure 1.1 for small examples of the special graphs that will be defined below. A complete graph of order $n$, denoted by $K_{n}$, is a graph of order $n$ in which every two vertices are adjacent. In particular, the complete graph $K_{3}$ is also called a triangle. With $S_{3}^{+}$we denote the unique graph of order 4 consisting of a triangle and one pendant edge. A path is a simple graph whose vertices can be arranged in a linear sequence in such a way that two vertices are adjacent if they are consecutive in the sequence, and are nonadjacent otherwise. A cycle on three or more vertices is a simple graph whose vertices can be arranged in a cyclic sequence in such a way that two vertices are adjacent if they are consecutive in the sequence, and are nonadjacent otherwise. The length of a 
path or a cycle is the number of its edges. A path on $n$ vertices and a cycle on $n$ vertices are denoted by $P_{n}$ and $C_{n}$, respectively. A graph $G$ is called bipartite if $V(G)$ can be partitioned into two disjoint sets $A$ and $B$ such that every edge of $G$ has one end-vertex in $A$ and one end-vertex in $B$. Note that a graph is bipartite if and only if it contains no cycle of odd length [110]. A complete bipartite graph $K_{a, b}$ is a bipartite graph with bipartition $(A, B)$ such that $|A|=a,|B|=b$, and every vertex in $A$ is adjacent to every vertex in $B$. In particular, the complete bipartite graph $K_{1, n}$ is also called a star.

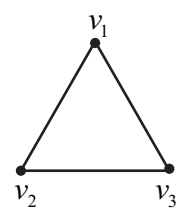

$K_{3}$

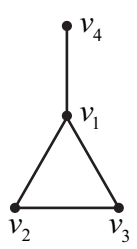

$S_{3}^{+}$

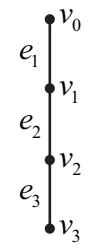

$P_{4}$

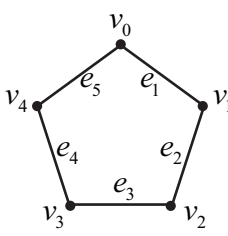

$C_{5}$

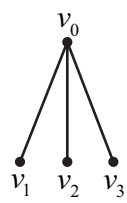

$K_{1,3}$

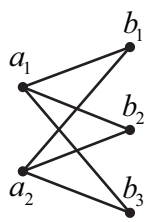

$K_{2,3}$

Figure 1.1: Examples of special graphs.

Given two graphs $G$ and $H, H$ is said to be a subgraph of $G$ if $V(H) \subseteq V(G)$ and $E(H) \subseteq E(G)$. If $H$ is a subgraph of $G$, then we say that $G$ contains $H$ or $H$ is contained in $G$, denoted by $G \supseteq H$ or $H \subseteq G$, respectively. A spanning subgraph of a graph $G$ is a subgraph with the same vertex set as $G$. For a subset $U \subseteq V(G)$, the subgraph of $G$ induced by $U$, denoted by $G[U]$, is the subgraph of $G$ whose vertex set is $U$ and whose edge set consists of all edges of $G$ which have both end-vertices in $U$. For $U \subset V(G)$, we let $G-U$ denote the subgraph of $G$ induced by $V(G) \backslash U$. In the special case when $U=\{u\}$, we simply write $G-u$ for $G-\{u\}$.

A graph is connected if, for every partition of its vertex set into two nonempty sets $A$ and $B$, there is an edge with one end-vertex in $A$ and one end-vertex in $B$; otherwise the graph is disconnected. Given two graphs $G$ and $H$, we say that $G$ and $H$ are disjoint if they have no common vertex. The disjoint union of two disjoint graphs $G$ and $H$, denoted by $G \cup H$, is the graph with vertex set $V(G) \cup V(H)$ and edge set $E(G) \cup E(H)$. Note that a disconnected graph $G$ can be viewed as the disjoint union of connected graphs, and each of these connected graphs is called a component of $G$. We use $n G$ to denote the disjoint union of $n$ copies of $G$. The join of two disjoint graphs $G$ 
and $H$, denoted by $G \vee H$, is the graph obtained from $G \cup H$ by adding edges joining every vertex of $G$ to every vertex of $H$.

A vertex cut of a connected graph $G$ is a subset $U \subset V(G)$ such that $G-U$ is disconnected. The vertex connectivity $\kappa(G)$ of a non-complete connected graph $G$ is the cardinality of a smallest vertex cut of $G$. For the complete graph $K_{n}$ (which has no vertex cut) it is defined by $\kappa\left(K_{n}\right)=n-1$, and for a disconnected graph it is zero. A graph $G$ is $k$-connected if $\kappa(G) \geq k$. Menger's Theorem [141] shows that a graph $G$ is $k$-connected if and only if for every two distinct vertices $u$ and $v$ of $G$, there are at least $k$ pairwise internally disjoint paths connecting $u$ and $v$ in $G$.

For a positive integer $n$, let $[n]=\{1,2, \ldots, n\}$. For any real number $x$, let $\lceil x\rceil$ be the smallest integer that is greater than or equal to $x$, and $\lfloor x\rfloor$ be the greatest integer that is less than or equal to $x$. In this thesis, we will make use of the standard Bachmann-Landau notation such as $O$ (also commonly known as Big O notation), $o, \Omega$ and $\Theta$ to indicate asymptotic growth rates of functions.

For an integer $k \geq 1$ and a graph $G$, we refer to a mapping $c: E(G) \rightarrow[k]$ as a $k$-edge-coloring (or just edge-coloring) of $G$. We call this ( $k$-)edge-coloring proper if any two distinct edges of $G$ that share an end-vertex are assigned different integers. If the mapping $c$ is surjective, then the $k$-edge-coloring $c$ is called an exact k-edge-coloring. For convenience, we sometimes use names of actual colors, like "red", "blue" or "green", instead of integers. A graph with an edge-coloring $c$ is denoted by $(G, c)$, and we also refer to it as an edge-colored graph. If the edge-coloring is clear, then we simply write $G$ for $(G, c)$. An edge-colored graph is called rainbow if all edges are colored differently, and monochromatic if all edges are colored the same. Given two graphs $G, H$ and an edge-coloring $c$ of $G$, we say that $(G, c)$ is rainbow $H$-free if $(G, c)$ contains no rainbow $H$. A Gallai-k-coloring is a rainbow triangle-free $k$-edge-coloring of a complete graph, i.e., in a Gallai-k-coloring of $K_{n}$ at most two distinct integers are assigned to the edges of every copy of $K_{3}$ in this edge-colored $K_{n}$.

Given an edge-colored graph $(G, c)$ and an edge $e \in E(G)$, we say that $c_{G}(e)$ (or simply $c(e)$ ) is the color used on (i.e., assigned to) the edge $e$. For $U$, $V \subseteq V(G)$ with $U \cap V=\emptyset$, we use $E(U, V)$ (respectively, $C(U, V)$ ) to denote 
the set of all edges between $U$ and $V$ (respectively, the set of colors used on these edges between $U$ and $V$ ). If all the edges in $E(U, V)$ are colored by a single color, then we use $c(U, V)$ to denote this color. In the special case when $U=\{u\}$, we simply write $E(u, V), C(u, V)$ and $c(u, V)$ for $E(\{u\}, V), C(\{u\}, V)$ and $c(\{u\}, V)$, respectively. We let $C(G[U])$ (or simply, $C(U)$ ) and $C(G-U)$ denote the set of colors used on $E(G[U])$ and $E(G-U)$, respectively. For a color $i$, the subgraph induced by color $i$ is the subgraph consisting of all the edges with color $i$ and all the vertices that are incident with at least one edge of color $i$.

Let $G$ be an edge-colored complete graph with vertex set $\left\{v_{1}, v_{2}, \ldots, v_{n}\right\}$, and $H_{1}, H_{2}, \ldots, H_{n}$ be $n$ pairwise disjoint edge-colored complete graphs. The blow-up $G\left(H_{1}, H_{2}, \ldots, H_{n}\right)$ of $G$ is an edge-colored complete graph with vertex set $\bigcup_{i=1}^{n} V\left(H_{i}\right)$ such that

- $c_{G\left(H_{1}, H_{2}, \ldots, H_{n}\right)}(x y)=c_{H_{i}}(x y)$ if $x, y \in V\left(H_{i}\right)$ for some $i \in[n]$,

- $c_{G\left(H_{1}, H_{2}, \ldots, H_{n}\right)}(x y)=c_{G}\left(v_{i} v_{j}\right)$ if $x \in V\left(H_{i}\right)$ and $y \in V\left(H_{j}\right)$ for some $1 \leq i \neq j \leq n$.

If $H_{1}=H_{2}=\cdots=H_{n}=H$, we will write $G(n \cdot H)$ for $G(H, H, \ldots, H)$. If $H_{1}=\cdots=H_{s}=H^{\prime}$ and $H_{s+1}=\cdots=H_{n}=H^{\prime \prime}$ for some $1 \leq s<n$, we will write $G\left(s \cdot H^{\prime},(n-s) \cdot H^{\prime \prime}\right)$ for $G\left(H^{\prime}, \ldots, H^{\prime}, H^{\prime \prime}, \ldots, H^{\prime \prime}\right)$. Similarly, we will use the abbreviation $G\left(s \cdot H^{\prime}, t \cdot H^{\prime \prime},(n-s-t) \cdot H^{\prime \prime \prime}\right)$.

We now have all the necessary ingredients to introduce the rich area of graph theory which is known under the common heading Ramsey theory.

\subsection{Ramsey theory}

Ramsey theory, named after the British mathematical logician Frank Plumpton Ramsey, is an important branch of extremal combinatorics. It can be considered as a generalization of the Pigeonhole Principle. Its origin reveals an important mathematical thought: complete disorder is impossible; any (large enough) structure will necessarily contain an orderly substructure [144]. The first Ramsey-type result mentioned in the literature is the Hilbert Cube Lemma [98], which was proved by David Hilbert in 1892. Since it would take 
considerable efforts and additional terminology to explain this lemma, and according to [162] it did not have much influence at the time, we refrain from stating it. We start this exposition with another two milestone Ramsey-type results that appeared before the birth of Ramsey's Theorem, namely Schur's Theorem and Van der Waerden's Theorem.

In 1916, motivated by Fermat's Last Theorem (which was a conjecture at that time), Issai Schur [161] proved that for any $n \geq 1$, the congruence $x^{n}+y^{n} \equiv z^{n}(\bmod p)$ has a nontrivial solution for all sufficiently large primes $p$. Schur's proof utilizes the following Ramsey-type theorem.

Theorem 1.1 (Schur's Theorem [161]). For any positive integer $k$, there exists a smallest positive integer $s(k)$ such that for all $n \geq s(k)$, every $k$-coloring of $[n]$ contains integers $a, b, c$ of the same color with $a+b=c$.

Here a $k$-coloring of $[n]$ is an assignment of one of $k$ distinct colors to every integer of $[n]$. In 1927, the Dutch mathematician Bartel Leendert van der Waerden [169] proved the following celebrated theorem on arithmetic progressions.

Theorem 1.2 (Van der Waerden's Theorem [169]). For any positive integers $k$ and $\ell$, there exists a smallest positive integer $w(\ell ; k)$ such that for all $n \geq$ $w(\ell ; k)$, every $k$-coloring of $[n]$ contains a monochromatic $\ell$-term arithmetic progression.

Here a monochromatic $\ell$-term arithmetic progression is a sequence in $[n]$ of the form $a, a+d, a+2 d, \ldots, a+(\ell-1) d$, where $d \in[n]$ and all entries in the sequence have the same color. We now introduce the well-known Ramsey's Theorem (finite version), which was proved by Ramsey [153] back in 1930.

Theorem 1.3 (Ramsey's Theorem [153]). For any positive integers $k$ and $m$, there exists a smallest positive integer $r_{k}(m)$ such that for all $n \geq r_{k}(m)$, every $k$-edge-coloring of $K_{n}$ contains a monochromatic copy of $K_{m}$.

The numbers $r_{k}(m)$ are called Ramsey numbers. The solution to the Party Problem in the introductory section of this chapter shows that $r_{2}(3) \leq 6$, 
and one easily shows by a suitable 2-edge-coloring of $K_{5}$ (two edge-disjoint monochromatic $C_{5} \mathrm{~s}$ ) that in fact equality holds.

Formally, given $k$ positive integers $m_{1}, m_{2}, \ldots, m_{k}$, the classical $k$-colored Ramsey number $r\left(m_{1}, m_{2}, \ldots, m_{k}\right)$ is defined to be the minimum integer $n$ such that every $k$-edge-coloring of $K_{n}$ contains a monochromatic copy of $K_{m_{i}}$ in color $i$ for some $i \in[k]$. In the special case when $m_{1}=m_{2}=\cdots=m_{k}=m$, we simply write $r_{k}(m)$ for $r(m, m, \ldots, m)$. The existence of $r\left(m_{1}, m_{2}, \ldots, m_{k}\right)$ is ensured by Ramsey's Theorem. Although the definition of Ramsey numbers is easy to understand and their existence is guaranteed, the exact values are extremely difficult to determine, even for some small integers $m_{1}, m_{2}, \ldots, m_{k}$. There are only nine exact values of nontrivial 2-colored Ramsey numbers known to date, as shown in Table 1.1. For multicolor Ramsey numbers, there are only two known nontrivial exact values: $r(3,3,3)=17$ (see [88]) and $r(3,3,4)=30$ (see [43]). The former value is the solution to the generalized Party Problem as it was given as an example in the layman's introduction of this chapter.

\begin{tabular}{|c|c|c|c|c|c|c|c|}
\hline$r(s, t) \backslash t$ & 3 & 4 & 5 & 6 & 7 & 8 & 9 \\
\hline$s$ & 6 & 9 & 14 & 18 & 23 & 28 & 36 \\
\hline 4 & & 18 & 25 & & & & \\
\hline
\end{tabular}

Table 1.1: All known nontrivial 2-colored Ramsey numbers.

In 1935, Erdős and Szekeres [68] rediscovered Ramsey's Theorem and proved an upper bound on off-diagonal Ramsey numbers: $r(s, t) \leq\left(\begin{array}{c}s+t-2 \\ s-1\end{array}\right)$. When $s$ is fixed and $t$ is large, Bohman-Keevash [20] and Li-Rousseau-Zang [118] proved the following lower and upper bounds, respectively.

$$
c_{s} t^{\frac{s+1}{2}}(\ln t)^{\frac{1}{s-2}-\frac{s+1}{2}} \leq r(s, t) \leq(1+o(1)) t^{s-1}(\ln t)^{2-s} .
$$

For the diagonal case, Erdős [56] proved that $r(t, t) \geq(1+o(1)) \frac{t}{\sqrt{2} e} 2^{t / 2}$ using the probabilistic method. Spencer [165] improved the term $\frac{1}{\sqrt{2}}$ to $\sqrt{2}$ and this is the best until now lower bound. For the upper bound, Conlon [44] proved that $r(t+1, t+1) \leq t^{-c \ln t / \ln \ln t}\left(\begin{array}{c}2 t \\ t\end{array}\right)$, which was improved to $r(t+1, t+1) \leq e^{-c^{\prime}(\ln t)^{2}}\left(\begin{array}{c}2 t \\ t\end{array}\right)$ by Sah [157] recently. For large values of $k$, 
the best known upper bound is $r_{k}(t) \leq k^{k t}$ which can be derived from the argument of Erdős and Szekeres [68]. Recently, Conlon and Ferber [46] proved a lower bound $r_{k}(t) \geq\left(2^{7 k / 24+C}\right)^{t-o(t)}$, where $C$ depends on the residue of $k$ modulo 3 . Soon afterwards, this lower bound was improved to $r_{k}(t) \geq\left(2^{3 k / 8-1 / 4}\right)^{t-o(t)}$ by Wigderson [174].

We next give the definition of graph Ramsey numbers, which was initiated in a series of papers due to Chvátal and Harary [37-40] of the 1970s. For $k$ graphs $H_{1}, H_{2}, \ldots, H_{k}$, the $k$-colored Ramsey number $r\left(H_{1}, H_{2}, \ldots, H_{k}\right)$ is defined to be the minimum integer $n$ such that every $k$-edge-coloring of $K_{n}$ contains a monochromatic copy of $H_{i}$ in color $i$ for some $i \in[k]$. In the special case when $H_{1}=H_{2}=\cdots=H_{k}=H$, we simply write $r_{k}(H)$. Note that $r\left(K_{m_{1}}, K_{m_{2}}, \ldots, K_{m_{k}}\right)=r\left(m_{1}, m_{2}, \ldots, m_{k}\right)$. For specific graphs, such as cycles, paths, stars, books, fans, wheels, trees and forests, considerable progress on determining graph Ramsey numbers has been obtained over the past few decades, including exact values, asymptotic values and bounds for these numbers. We refer the interested reader to the dynamic survey [152] and the monograph of Graham, Rothschild and Spencer [87] for more details.

Finally, we briefly introduce some applications of Ramsey theory. Ramseytype results have close connections to various fields in mathematics and other subject areas, including number theory, algebra, geometry, logic, topology, ergodic theory, functional analysis, the theory of ultrafilters, set theory, information theory and theoretical computer science [156]. Typical results in number theory include Schur's Theorem and Van der Waerden's Theorem, as well as Szemerédi's Theorem (see Theorem 7.1). The result of Erdős and Szekeres [68] has had a great influence on geometry. Several Ramsey-type results in ergodic theory can be found in the book of Furstenberg [84]. Ramsey theory has also been massively applied in information theory, especially with respect to communication channels; see the survey of Roberts [154]. In theoretical computer science, Ramsey theory has vast applications in different problem areas, such as the information retrieval problem [176], Boolean function computation [2] and order invariant decision trees [142]. For more information on applications of Ramsey theory, we refer the reader to two excellent surveys $[149,156]$ and the references therein. 


\subsection{Generalizations of Ramsey theory}

Since Ramsey published his pioneering paper [153], many variants and generalizations of Ramsey numbers have been considered, such as hypergraph Ramsey numbers [50], anti-Ramsey numbers [67], rainbow Ramsey numbers [70], ordered Ramsey numbers [8,49], the Erdős-Hajnal Conjecture [63], Gallai-Ramsey numbers and the Erdős-Gyárfás function. In this section, we will introduce Gallai-Ramsey numbers and the Erdős-Gyárfás function, as they play a key role in parts of the thesis.

\subsubsection{Gallai-Ramsey theory}

Recall that a Gallai-coloring is a rainbow triangle-free edge-coloring of a complete graph, so no $K_{3}$ in the colored complete graph is allowed to have all its edges colored differently. The term Gallai-coloring was first used by Gyárfás and Simonyi [92] in honor of Tibor Gallai's decomposition theorem for rainbow triangle-free colorings [85], but the study of Gallai-colorings has arisen in a wide range of areas, such as poset theory [85], the Erdős-Hajnal Conjecture [75], the rainbow Erdős-Rothschild problem [10,17], information theory $[111,112]$, perfect graph theory [31] and other studies of Ramsey-type problems $[91,93]$.

Gallai [85] gave the following characterization of the structure of a complete graph with a Gallai-coloring.

Theorem 1.4 (Gallai $[85,92,135])$. In any Gallai-coloring of a complete graph, the vertex set can be partitioned into nonempty sets $V_{1}, V_{2}, \ldots, V_{m}$ with $m \geq 2$ such that

(1) for any pair $i, j$ with $1 \leq i<j \leq m$, there is only one color on the edges between $V_{i}$ and $V_{j}$, and

(2) there are at most two colors on the edges in $\bigcup_{1 \leq i<j \leq m} E\left(V_{i}, V_{j}\right)$.

We call a vertex partition as given by the statement in Theorem 1.4 a Gallai partition. Given a Gallai-coloring of a complete graph and a Gallai partition $V_{1}, V_{2}, \ldots, V_{m}$, with a chosen $v_{i} \in V_{i}$ for every $i \in[m]$, the subgraph induced 
by $\left\{v_{1}, v_{2}, \ldots, v_{m}\right\}$ is called the reduced graph of this partition. Although this reduced graph is clearly a 2-edge-colored complete graph, the above result does not give us any information about the cardinality of each set in the partition or the coloring within each part, except that these colorings are rainbow triangle-free.

In 2010, Gyárfás et al. [91] introduced the following restricted Ramsey number $R G(k, H)$. This is the smallest positive integer $n$ such that in every Gallai- $k$-coloring of $K_{n}$, there is a monochromatic copy of the graph $H$. The term Gallai-Ramsey number was first used by Faudree et al. [72] in the same year. We now give the definition of the Gallai-Ramsey number. For graphs $H_{1}, H_{2}, \ldots, H_{k}$ and a graph $G$, the $k$-colored Gallai-Ramsey number $\operatorname{gr}\left(G: H_{1}, H_{2}, \ldots, H_{k}\right)$ is defined to be the minimum integer $n$ such that every rainbow $G$-free $k$-edge-coloring of $K_{n}$ contains a monochromatic copy of $H_{i}$ in color $i$ for some $i \in[k]$. In the special case when $H_{1}=H_{2}=\cdots=H_{k}=H$, we simply write $g r_{k}(G: H)$. Equivalently, $g r_{k}(G: H)$ is the minimum integer $n$ such that every $k$-edge-coloring of $K_{n}$ contains either a rainbow copy of $G$ or a monochromatic copy of $H$. It is clear that $g r_{k}(G: H) \leq r_{k}(H)$ for any two graphs $G$ and $H$.

In the past decade, there has been a great development in the study of Gallai-Ramsey numbers. Most of these works focus on the case when $G$ is a triangle $[93,123], S_{3}^{+}[80,119,120]$ or a path $[117,121]$. The following general results for $g r_{k}(G: H)$ were given by Gyárfás et al. [91] for $G=K_{3}$, and by Fujita and Magnant [80] for $G=S_{3}^{+}$.

Theorem 1.5 (Gyárfás et al. [91], Fujita and Magnant [80]). Let $G \in\left\{K_{3}, S_{3}^{+}\right\}$. If $H$ is not bipartite, then $g r_{k}(G: H)$ is exponential in $k$. If $H$ is bipartite but not a star, then $g r_{k}(G: H)$ is linear in $k$.

In [75], Fox, Grinshpun and Pach posed the following conjecture on an expression for the Gallai-Ramsey numbers of complete graphs in terms of their 2-colored Ramsey numbers. 
Conjecture 1.1 (Fox, Grinshpun and Pach [75]). For integers $k \geq 1$ and $t \geq 3$

$$
g r_{k}\left(K_{3}: K_{t}\right)= \begin{cases}\left(r_{2}\left(K_{t}\right)-1\right)^{k / 2}+1, & \text { if } k \text { is even, } \\ (t-1) \cdot\left(r_{2}\left(K_{t}\right)-1\right)^{(k-1) / 2}+1, & \text { if } k \text { is odd. }\end{cases}
$$

The cases with $t=3$ and $t=4$ of the above conjecture were verified in $[36,91]$ and [123], respectively. The cases for $t \geq 5$ are still open. There are also a lot of results on Gallai-Ramsey numbers for other monochromatic subgraphs, such as bipartite graphs $[34,122,175]$, cycles and paths $[23,26$, $34,78,93,116,164,172,177,178]$, stars [91,119], fans [138], wheels [163], double stars [106], books [181] and other specific graphs [139,173,179,180]. Gallai-Ramsey numbers have also been generalized to hypergraphs; see [27]. For more results on this topic, we refer the reader to two surveys $[82,83]$ and a book [137]. In Section 3.4, we give a short survey on known Gallai-Ramsey numbers for rainbow triangles and some open problems.

\subsubsection{The Erdős-Gyárfás function}

Let $p$ and $q$ be positive integers with $2 \leq q \leq\left(\begin{array}{l}p \\ 2\end{array}\right)$. An edge-coloring of the complete graph $K_{n}$ is said to be a $(p, q)$-coloring if every $K_{p}$ receives at least $q$ distinct colors. The function $f(n, p, q)$ is defined to be the minimum number of colors that are needed for $K_{n}$ to have a $(p, q)$-coloring. This function was first introduced by Erdős and Shelah [58,59], but Erdős and Gyárfás [62] were the first to study it in depth; it is now known as the Erdős-Gyárfás function. This function generalizes the multicolored Ramsey number, since determining $f(n, p, 2)$ is equivalent to determining the Ramsey number of $K_{p}$. Using the lower bound on $r_{k}(p)$, we have that $f(n, p, 2)$ is at most logarithmic in $n$. Moreover, if $p \geq 4$, then $f\left(n, p,\left(\begin{array}{l}p \\ 2\end{array}\right)\right)=\left(\begin{array}{l}n \\ 2\end{array}\right)$.

Erdős and Gyárfás [62] studied the behavior of $f(n, p, q)$ when $q$ varies from $\left(\begin{array}{l}p \\ 2\end{array}\right)$ to 2 . They proved that the smallest $q$ such that $f(n, p, q)$ is quadratic in $n$ is $\left(\begin{array}{c}p \\ 2\end{array}\right)-\left\lfloor\frac{p}{2}\right\rfloor+2$ for $p \geq 4$, and the smallest $q$ such that $f(n, p, q)$ is linear in $n$ is $\left(\begin{array}{l}p \\ 2\end{array}\right)-p+3$. Erdős and Gyárfás also showed that $f(n, p, p)$ is polynomial in $n$, while Conlon et al. [48] proved that $f(n, p, p-1)$ is subpolynomial in $n$. 
Thus the smallest $q$ such that $f(n, p, q)$ is polynomial in $n$ equals $p$.

The exact value of the Erdős-Gyárfás function is very difficult to determine, even for some small values of $p$ and $q$. For example, the best known lower bound for $f(n, 4,3)$ is $O(\log n)$ [77], while the best until now upper bound is $e^{O(\sqrt{\log n})}$ [145]. There is clearly a large gap between the lower and upper bound. On the other hand, some special cases of this function are closely related to other interesting problems. For example, $f(n, 9,34)$ relates to a Turán type hypergraph problem posed by Brown, Erdős and Sós [25, 62], $f(n, 5,9)$ relates to sets containing no 3-term arithmetic progression [3], and $f(n, 3,3)$ and $f(n, 5,9)$ relate to some problems on properly colored complete graphs $[62,155]$.

In 2000, Axenovich, Füredi and Mubayi [5] defined $r(G, H, q)$ to be the minimum number of colors in an edge-colored graph $G$ such that every subgraph $H$ receives at least $q$ colors, and they studied $r\left(K_{n, n}, K_{p, p}, q\right)$ systematically. In [114], Krueger investigated the asymptotic behavior of $r\left(K_{n}, P_{m}, q\right)$. Motivated by the additive energy in additive combinatorics, Pohoata and Sheffer [151] introduced the Color Energy Method. Using this method, they studied the Erdős-Gyárfás function, as well as a problem of distinct distances that is related to the Erdős distinct distances problem $[55,89]$ in discrete geometry. Recently, this method was further developed by Fish-PohoataSheffer [73] and Balogh-English-Heath-Krueger [9]. The Erdős-Gyárfás function was also studied in the setting of hypergraphs, see [47,147]. In [76], Fox, Pach and Suk studied a semi-algebraic variant of the Erdős-Gyárfás function. For more information on this topic, we refer the interested reader to $[1,6,7,30,33,54,113,146,158,159]$, Section 3.5 .1 of [51] and Section 7 of $[148]$.

\subsection{Outline of the main results of this thesis}

Apart from this introductory chapter, this thesis contains six technical chapters that are based on earlier submitted papers. In these chapters, we mainly concentrate on determining exact values of Gallai-Ramsey numbers of graphs. We also consider some related problems, such as Gallai-Ramsey multiplicity, 
the Erdős-Gyárfás function and the rainbow Erdős-Rothschild problem. The remainder of this thesis is organized as follows.

In Chapter 2, we consider the Gallai-Ramsey number for a rainbow triangle and monochromatic subgraphs $H$, where $H$ belongs to a class of connected graphs with five vertices and at most six edges. There are in total thirteen graphs in this graph class, and the Gallai-Ramsey numbers for eight of them have been studied step by step in several papers by others. We determine the exact values of the Gallai-Ramsey number for all the remaining five graphs. In addition, we also obtain Ramsey numbers and Gallai-Ramsey numbers for a class of unicyclic graphs $F_{2, n}$, where $F_{2, n}$ is the graph obtained by adding $n-2$ pendant edges to a single vertex of a $C_{4}$.

In Chapter 3, we determine the exact value of the Gallai-Ramsey number for a rainbow triangle and a monochromatic $K_{4}+e$, where $K_{4}+e$ is the graph on five vertices consisting of a $K_{4}$ with one pendant edge. For an integer $s$ with $0 \leq s \leq k$, if $H_{1}=\cdots=H_{s}=K_{4}+e$ and $H_{s+1}=\cdots=H_{k}=K_{3}$, then we will write $g r_{k}\left(K_{3}: s \cdot K_{4}+e,(k-s) \cdot K_{3}\right)$ for $g r\left(K_{3}: H_{1}, H_{2}, \ldots, H_{k}\right)$. We in fact determine the exact values of $g r_{k}\left(K_{3}: s \cdot K_{4}+e,(k-s) \cdot K_{3}\right)$ for all $0 \leq s \leq k$.

In Chapter 4, we first consider the maximum number of edges that are not contained in any rainbow triangle or monochromatic triangle in a $k$-edgecoloring of $K_{n}$. We determine a lower bound on this number using a variant of the Gallai-Ramsey number, and obtain an upper bound using Szemerédi's Regularity Lemma. Secondly, we consider the Gallai-Ramsey multiplicity problem for rainbow and monochromatic triangles. For $n \geq g r_{k}\left(K_{3}: K_{3}\right)$, we give upper and lower bounds for the minimum number of monochromatic triangles in a Gallai- $k$-coloring of $K_{n}$, yielding the exact value for $k=3$ and sufficiently large $n$.

In Chapter 5, we consider the Erdős-Gyárfás function within the framework of Gallai-colorings. We use the function $g(n, p, q)$ to denote the minimum number of colors needed for a Gallai- $(p, q)$-coloring of $K_{n}$. Using the antiRamsey number for $K_{3}$, we have that $g(n, p, q)$ is nontrivial only for $2 \leq$ $q \leq p-1$. Note that determining $g(n, p, 2)$ is equivalent to determining the Gallai-Ramsey number $g r_{k}\left(K_{3}: K_{p}\right)$. We give a general lower bound on $g(n, p, q)$ and we study how this function falls off from being equal to $n-1$ 
when $q=p-1$ and $p \geq 4$ to being $\Theta(\log n)$ when $q=2$. In particular, for appropriate $p$ and $n$, we prove that $g=n-c$ when $q=p-c$ and $c \in\{1,2\}$, $g \leq\left\lceil n^{1 / 2}\right\rceil-1$ when $q=\left\lfloor(p-1)^{1 / 2}\right\rfloor$, and $g$ is logarithmic in $n$ when $2 \leq q \leq\left\lceil\log _{2} p\right\rceil$. These results provide an upper bound on $g r_{k}\left(K_{3}: K_{p}\right)$.

In Chapter 6, we consider a Gallai-Ramsey-type problem for highly connected subgraphs. Recall that the Gallai-Ramsey number $g r_{k}(G: H)$ is the smallest integer $n$ such that every rainbow $G$-free $k$-edge-coloring contains a monochromatic copy of $H$. Instead of dealing with a fixed subgraph $H$, we consider the problem for a general $k$-connected (monochromatic) subgraph. To be more precise, we study the forbidden rainbow subgraph condition for a large $k$-connected monochromatic subgraph in an edge-colored complete graph. We also study an analogous problem in which the host graphs are complete bipartite graphs. Furthermore, we consider determining the order of the largest $k$-connected 2-edge-colored subgraph in a Gallai-3-coloring.

In Chapter 7, we study the rainbow Erdős-Rothschild problem with respect to 3 -term arithmetic progressions. Integer colorings without rainbow 3-term arithmetic progressions generalize edge-colorings without rainbow triangles. We obtain the asymptotic number of $r$-colorings of [ $n$ ] without rainbow 3term arithmetic progressions using the Hypergraph Container Method, and we show that the typical colorings with this property are 2-colorings. We also prove that $[n]$ attains the maximum number of rainbow 3-term arithmetic progression-free $r$-colorings among all subsets of $[n]$. Moreover, the exact number of rainbow 3 -term arithmetic progression-free $r$-colorings of $\mathbb{Z}_{p}$ is obtained, where $p$ is any prime and $\mathbb{Z}_{p}$ is the cyclic group of order $p$. 


\section{Chapter 2}

\section{Gallai-Ramsey numbers for a class of graphs with five vertices}

In this chapter, we consider the Gallai-Ramsey number $g r_{k}\left(K_{3}: H\right)$, where $H$ is a connected graph with five vertices and at most six edges. There are in total thirteen different choices for the graph $H$, and the Gallai-Ramsey numbers for eight of them have been determined step by step in several papers by others. In this chapter, we determine the exact value of the Gallai-Ramsey number for all the remaining five graphs. In addition, we obtain some related results for a class of unicyclic graphs.

\subsection{Introduction}

Recall that the Gallai-Ramsey number $g r_{k}(G: H)$ is the minimum integer $n$ such that every $k$-edge-coloring of $K_{n}$ contains either a rainbow copy of $G$ or a monochromatic copy of $H$. We start by remarking that determining the exact values of $g r_{k}(G: H)$ is far from trivial, even for choices of $G$ and $H$ with a small number of vertices and edges. The Gallai-Ramsey numbers $g r_{k}\left(K_{3}: H\right)$ for all different choices of a graph $H$ on four vertices can be found in several 
earlier papers. However, these numbers have not been established yet for several graphs with five vertices, and determining the exact values for these graphs is of fundamental importance in this research area. In this chapter, we mainly focus on studying $g r_{k}\left(K_{3}: H\right)$, where $H$ belongs to the class of connected graphs with five vertices and at most six edges. There are thirteen graphs in this graph class (see Figure 2.1 for an illustration of the graphs $F_{1}$ $F_{13}$ ). The Gallai-Ramsey numbers for $F_{1}, F_{2}, \ldots, F_{8}$ have been determined by Faudree et al. [72] for $F_{1}$ and $F_{2}$, Gyárfás et al. [91] for $F_{3}$, Wang et al. [173] for $F_{4}$ and $F_{5}$, Fujita and Magnant [78] for $F_{6}$, Wu et al. [175] for $F_{7}$, and Mao et al. [138] for $F_{8}$. In the subsequent sections, we will determine the Gallai-Ramsey numbers for all the remaining graphs in this class.

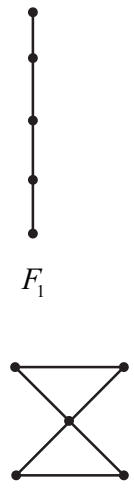

$F_{8}$

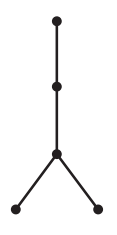

$F_{2}$

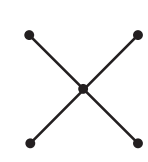

$F_{3}$

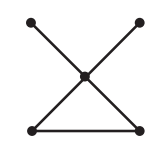

$F_{4}$

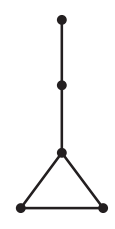

$F_{5}$

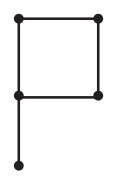

$F_{11}$

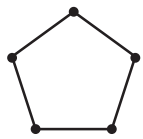

$F_{6}$

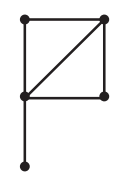

$F_{12}$

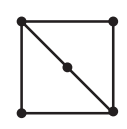

$F_{7}$

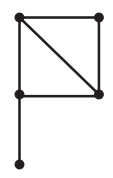

$F_{13}$

Figure 2.1: All connected graphs with five vertices and at most six edges.

For the graphs $F_{9}$ and $F_{10}$, we will prove the following result in Section 2.2.

Theorem 2.1. For any integer $k \geq 1$, we have

$$
g r_{k}\left(K_{3}: F_{9}\right)=g r_{k}\left(K_{3}: F_{10}\right)= \begin{cases}8 \cdot 5^{(k-2) / 2}+1, & \text { if } k \text { is even } \\ 4 \cdot 5^{(k-1) / 2}+1, & \text { if } k \text { is odd }\end{cases}
$$

For the graph $F_{11}$, we will prove that $g r_{k}\left(K_{3}: F_{11}\right)=k+4$ in a more general form in Section 2.3. In fact, we shall consider the Ramsey numbers and Gallai-Ramsey numbers for a class of unicyclic graphs $F_{2, n}$. For $n \geq 3$, let $F_{2, n}$ denote the graph obtained by adding $n-2$ pendant edges to a single 
vertex of a $C_{4}$. The vertex with degree $n$ is called the center of $F_{2, n}$. It is easy to see that $\left|V\left(F_{2, n}\right)\right|=\left|E\left(F_{2, n}\right)\right|=n+2$. Note that $F_{2,3}=F_{11}$. To resolve the Gallai-Ramsey numbers for this class of unicyclic graphs, we will prove the following two results in Section 2.3.

Theorem 2.2. For any integer $n \geq 3, r_{2}\left(F_{2, n}\right)=2 n-\varepsilon$, where $\varepsilon=1$ if $n$ is even, and $\varepsilon=0$ otherwise.

Theorem 2.3. Let $k$ and $n$ be two positive integers.

(1) If $k \geq 1$ and $n \in\{3,4\}$, then $g r_{k}\left(K_{3}: F_{2, n}\right)=r_{2}\left(F_{2, n}\right)+k-2$.

(2) If $k \geq 3$, then $g r_{k}\left(K_{3}: F_{2,5}\right)=k+9$.

(3) If $k \geq 3$ and $n \geq 6$, then

$$
k(n-1)+2 \geq g r_{k}\left(K_{3}: F_{2, n}\right) \geq \begin{cases}5 n / 2+k-6, & \text { if } n \text { is even, } \\ (5 n-1) / 2+k-4, & \text { if } n \text { is odd } .\end{cases}
$$

For the graphs $F_{12}$ and $F_{13}$, we will prove the following result in Section 2.4.

Theorem 2.4. For any integer $k \geq 1$, we have

$$
g r_{k}\left(K_{3}: F_{12}\right)=g r_{k}\left(K_{3}: F_{13}\right)= \begin{cases}9 \cdot 5^{(k-2) / 2}+1, & \text { if } k \text { is even, } \\ 4 \cdot 5^{(k-1) / 2}+1, & \text { if } k \text { is odd } .\end{cases}
$$

\subsection{Gallai-Ramsey numbers for $F_{9}$ and $F_{10}$}

In this section, we will prove Theorem 2.1. We begin with the following simple results which will be used later.

Proposition 2.5. If $H_{1}$ is a subgraph of $H_{2}$, then $g r_{k}\left(K_{3}: H_{1}\right) \leq g r_{k}\left(K_{3}: H_{2}\right)$. Theorem 2.6 (Hendry [97]). $r_{2}\left(F_{9}\right)=r_{2}\left(F_{10}\right)=9$.

We shall use the following known result about the Gallai-Ramsey number $g r_{k}\left(K_{3}: K_{3}\right)$, and this result will also be used in Chapters 3, 4 and 5 . 
Theorem 2.7 (Chung and Graham [36], Gyárfás et al. [91]). For any integer $k \geq 1$, we have

$$
g r_{k}\left(K_{3}: K_{3}\right)= \begin{cases}5^{k / 2}+1, & \text { if } k \text { is even, } \\ 2 \cdot 5^{(k-1) / 2}+1, & \text { if } k \text { is odd }\end{cases}
$$

Recall that the complete bipartite graph $K_{1, n}(n \geq 1)$ is also called a star. In the sequel, we shall call an isolated vertex (i.e., a vertex with degree 0 ) a trivial star.

Since $F_{9}$ is a subgraph of $F_{10}$, we have $g r_{k}\left(K_{3}: F_{9}\right) \leq g r_{k}\left(K_{3}: F_{10}\right)$ by Proposition 2.5. Thus Theorem 2.1 follows immediately from the following two lemmas, the first of which provides lower bounds for $g r_{k}\left(K_{3}: F_{9}\right)$, and the second of which provides the matching upper bounds for $g r_{k}\left(K_{3}: F_{10}\right)$.

Lemma 2.8. For any integer $k \geq 1$, we have

$$
g r_{k}\left(K_{3}: F_{9}\right)> \begin{cases}8 \cdot 5^{(k-2) / 2}, & \text { if } k \text { is even, } \\ 4 \cdot 5^{(k-1) / 2}, & \text { if } k \text { is odd. }\end{cases}
$$

Proof. As usual, the proof of the lower bounds is by construction. If $k$ is even, then let $G_{2}$ be a 2-edge-colored $K_{8}$ which contains no monochromatic $F_{9}$, using colors 1 and 2 (such $G_{2}$ exists by Theorem 2.6). Suppose that $2 i<k$ and we have constructed a (2i)-edge-coloring $G_{2 i}$ of $K_{n_{2 i}}$ containing neither a rainbow $K_{3}$ nor a monochromatic $F_{9}$, where $n_{2 i}:=8 \cdot 5^{(2 i-2) / 2}$. Let $G^{\prime}$ be a 2-edge-colored $K_{5}$ using colors $2 i+1$ and $2 i+2$ which contains no monochromatic $K_{3}$, i.e., colors $2 i+1$ and $2 i+2$ induce two monochromatic copies of $C_{5}$. We construct $G_{2 i+2}$ such that $G_{2 i+2}$ is the blow-up $G^{\prime}\left(5 \cdot G_{2 i}\right)$ of $G^{\prime}$. Finally, we obtain a $k$-edge-coloring $G_{k}$ of $K_{n}$ containing neither a rainbow $K_{3}$ nor a monochromatic $F_{9}$, where $n=8 \cdot 5^{(k-2) / 2}$.

If $k$ is odd, then let $G_{1}$ be a monochromatic $K_{4}$ using color 1 . Suppose that $2 i-1<k$ and we have constructed a $(2 i-1)$-edge-coloring $G_{2 i-1}$ of $K_{n_{2 i-1}}$ which contains neither a rainbow $K_{3}$ nor a monochromatic $F_{9}$, where $n_{2 i-1}:=4 \cdot 5^{(2 i-2) / 2}$. Let $G^{\prime \prime}$ be a 2-edge-colored $K_{5}$ using colors $2 i$ and $2 i+1$ which contains no monochromatic $K_{3}$. We construct $G_{2 i+1}$ such that $G_{2 i+1}$ is the blow-up $G^{\prime \prime}\left(5 \cdot G_{2 i-1}\right)$ of $G^{\prime \prime}$. Finally, we obtain a $k$-edge-coloring $G_{k}$ of 
$K_{n}$ with $n=4 \cdot 5^{(k-1) / 2}$ containing neither a rainbow $K_{3}$ nor a monochromatic $F_{9}$.

Lemma 2.9. For any integer $k \geq 1$, we have

$$
g r_{k}\left(K_{3}: F_{10}\right) \leq \begin{cases}8 \cdot 5^{(k-2) / 2}+1, & \text { if } k \text { is even, } \\ 4 \cdot 5^{(k-1) / 2}+1, & \text { if } k \text { is odd. }\end{cases}
$$

Proof. We prove the statement by induction on $k$. The case $k=1$ is trivial and the case $k=2$ holds by Theorem 2.6, so we may assume $k \geq 3$. Suppose the statement holds for all $k^{\prime}<k$, and we will prove it for $k$. For a contradiction, suppose that $G$ is a Gallai- $k$-coloring of $K_{n}$ containing no monochromatic $F_{10}$, where

$$
n:= \begin{cases}8 \cdot 5^{(k-2) / 2}+1, & \text { if } k \text { is even, } \\ 4 \cdot 5^{(k-1) / 2}+1, & \text { if } k \text { is odd. }\end{cases}
$$

Since $k \geq 3$, we have $n \geq 21$. By Theorem 1.4, there exists a Gallai partition $V_{1}, V_{2}, \ldots, V_{m}$ with $m \geq 2$. We choose such a partition such that $\left|V_{1}\right| \geq\left|V_{2}\right| \geq$ $\cdots \geq\left|V_{m}\right|$ and $m$ is minimal. Since $r_{2}\left(F_{10}\right)=9$, we have $2 \leq m \leq 8$. Assume that colors 1 and 2 are the two colors used between the parts (if there is only one color used between the parts, then let color 1 be this color). We first have the following simple facts since $G$ is monochromatic $F_{10}$-free.

Fact 2.1. Let $U, V$ be two nonempty subsets of $V(G)$ with $U \cap V=\emptyset$ and $c(U, V)=c_{0}$, where $c_{0} \in[k]$. Then

(1) there is no monochromatic $P_{4}$ using color $c_{0}$ within $U$ or $V$, respectively;

(2) if $|U| \geq 3$ and $|V| \geq 2$, then $c_{0} \notin C(U)$;

(3) if $|U| \geq 2$ and $|V| \geq 2$, then there is no vertex with color $c_{0}$ to both $U$ and $V$ in $V(G) \backslash(U \cup V)$.

Claim 2.1. We have $4 \leq m \leq 8$, and $C\left(V_{i}, \bigcup_{j \in[m] \backslash\{i\}} V_{j}\right)=\{1,2\}$ for every $i \in[m]$.

Proof. Suppose $2 \leq m \leq 3$. If $m=3$, then at least two of the colors $c\left(V_{1}, V_{2}\right)$, $c\left(V_{1}, V_{3}\right)$ and $c\left(V_{2}, V_{3}\right)$ are the same color, say $c\left(V_{1}, V_{2}\right)=c\left(V_{1}, V_{3}\right)$. This 
implies that $V_{1}$ and $V(G) \backslash V_{1}$ form a Gallai partition with exactly two parts, contradicting the minimality of $m$.

Thus $m=2$ and $c\left(V_{1}, V_{2}\right)=1$. If $\left|V_{2}\right| \geq 2$, then $1 \notin C\left(V_{1}\right)$ by Fact 2.1 (2) and since $n \geq 21$. Applying induction on $k$ within $V_{1}$, we have $|V(G)|=$ $\left|V_{1}\right|+\left|V_{2}\right| \leq 2\left|V_{1}\right| \leq 2\left(g r_{k-1}\left(K_{3}: F_{10}\right)-1\right)<n$, a contradiction. Thus $\left|V_{2}\right|=1$ and $\left|V_{1}\right|=n-1$. By Fact 2.1 (1), color 1 induces a subgraph $G^{(1)}$ such that each component is a $K_{3}$ or a star within $V_{1}$. In order to avoid a rainbow $K_{3}$ and a monochromatic $P_{4}$ in color 1 , there is only a single color between each pair of components. By Theorem 2.7, we have

$$
g r_{k-1}\left(K_{3}: K_{3}\right)= \begin{cases}2 \cdot 5^{(k-2) / 2}+1, & \text { if } k \text { is even, } \\ 5^{(k-1) / 2}+1, & \text { if } k \text { is odd. }\end{cases}
$$

Note that $F_{10}$ is a subgraph of $K_{1,3,3}$ and $K_{2,2,2}$. Since $\left|V_{1}\right|$ is not divisible by 3 , $G^{(1)}$ contains at most $n_{k}:=g r_{k-1}\left(K_{3}: K_{3}\right)-2$ components each of which is a $K_{3}$. For $0 \leq i \leq n_{k}$, if there are exactly $n_{k}-i$ components each of which is a $K_{3}$, then there are at most $i+1$ components each of which is a nontrivial star; otherwise there is a monochromatic $K_{2,2,2}$. Thus after removing at most $2\left(n_{k}-i\right)+i+1$ vertices, there is no edge using color 1 within $V_{1}$. Thus $|V(G)|=\left|V_{1}\right|+\left|V_{2}\right| \leq 2\left(n_{k}-i\right)+i+1+\left(g r_{k-1}\left(K_{3}: F_{10}\right)-1\right)+1<n$, a contradiction. Therefore, $4 \leq m \leq 8$.

If $\left|C\left(V_{i}, \bigcup_{j \in[m] \backslash\{i\}} V_{j}\right)\right|=1$ for some $i \in[m]$, then $V_{i}$ and $V(G) \backslash V_{i}$ form a Gallai partition with exactly two parts, contradicting the minimality of $m$.

By Claim 2.1, we have $4 \leq m \leq 8$. Let $r$ be the number of parts with at least three vertices, i.e., $\left|V_{r}\right| \geq 3$ and $\left|V_{r+1}\right| \leq 2$ (here we define $\left|V_{m+1}\right|=0$ ). Since $m \leq 8$ and $|V(G)| \geq 21$, we have $1 \leq r \leq m \leq 8$. We divide the rest of the proof into four cases based on the number $r$.

Case 1. $r=1$.

For $i \in\{1,2\}$, let $A_{i}$ be the union of parts with color $i$ to $V_{1}$. By Claim 2.1, $A_{1}$ and $A_{2}$ are two nonempty sets and at least one of $A_{1}$ and $A_{2}$ contains more than one vertex, say $\left|A_{1}\right| \geq 2$. By Fact 2.1 (2), we have $1 \notin C\left(V_{1}\right)$. Thus $\left|V_{1}\right| \leq g r_{k-1}\left(K_{3}: F_{10}\right)-1$. Moreover, since $2 \geq\left|V_{2}\right| \geq \cdots \geq\left|V_{m}\right|$ and $m \leq 8$, we have $\left|A_{1}\right|+\left|A_{2}\right| \leq 14$. If $\left|A_{2}\right| \geq 2$, then $2 \notin C\left(V_{1}\right)$, which implies 
$|V(G)|=\left|V_{1}\right|+\left|A_{1}\right|+\left|A_{2}\right| \leq g r_{k-2}\left(K_{3}: F_{10}\right)-1+14<n$, a contradiction. Thus $\left|A_{2}\right|=1$. We further have $\left|A_{1}\right| \geq 3$; otherwise $|V(G)|=\left|V_{1}\right|+\left|A_{1}\right|+\left|A_{2}\right| \leq$ $g r_{k-1}\left(K_{3}: F_{10}\right)-1+2+1<n$. By Fact $2.1(2)$, we have $1 \notin C\left(A_{1}\right)$, which implies $\left|A_{1}\right| \leq g r_{k-1}\left(K_{3}: F_{10}\right)-1$. Therefore, $|V(G)|=\left|V_{1}\right|+\left|A_{1}\right|+\left|A_{2}\right| \leq$ $g r_{k-1}\left(K_{3}: F_{10}\right)-1+\min \left\{g r_{k-1}\left(K_{3}: F_{10}\right)-1+1,14\right\}<n$, a contradiction.

Case 2. $r=2$.

In this case, we may assume that $c\left(V_{1}, V_{2}\right)=1$. Then by Fact 2.1 (2), for $i \in\{1,2\}$, we have $1 \notin C\left(V_{i}\right)$ and thus $\left|V_{i}\right| \leq g r_{k-1}\left(K_{3}: F_{10}\right)-1$. Recall that $m \leq 8$ and $2 \geq\left|V_{3}\right| \geq \cdots \geq\left|V_{m}\right|$. If $\left|V_{6}\right| \geq 2$, then there is a monochromatic $K_{2,2,2}$ by $r_{2}\left(K_{3}\right)=6$, contradicting Fact 2.1 (3). Thus $\left|V_{3} \cup \cdots \cup V_{m}\right| \leq 9$. By Fact 2.1 (3), we also have that there is no vertex with color 1 to both $V_{1}$ and $V_{2}$. Thus we can partition $V_{3} \cup \cdots \cup V_{m}$ into three parts $A, B$ and $C$ such that, $c\left(A, V_{1}\right)=c\left(C, V_{2}\right)=1$ and $c\left(A, V_{2}\right)=c\left(C, V_{1}\right)=c\left(B, V_{1} \cup V_{2}\right)=2$. If $|A \cup B| \geq 2$ and $|C \cup B| \geq 2$, then for $i \in\{1,2\}$, we have $2 \notin C\left(V_{i}\right)$ and thus $|V(G)|=\left|V_{1}\right|+\left|V_{2}\right|+\left|V_{3} \cup \cdots \cup V_{m}\right| \leq 2\left(g r_{k-2}\left(K_{3}: F_{10}\right)-1\right)+9<n$, a contradiction. Thus $|A \cup B| \leq 1$ or $|C \cup B| \leq 1$, say $|C \cup B| \leq 1$, so $|B| \leq 1$.

We first suppose $|B|=1$ and $|C|=0$. Since $m \geq 4$, we have $|A| \geq 1$, and thus $2 \notin C\left(V_{2}\right)$ by Fact 2.1 (2). Since $|A|=|V(G)|-\left|V_{1}\right|-\left|V_{2}\right|-|B| \geq$ $n-\left(g r_{k-1}\left(K_{3}: F_{10}\right)-1\right)-\left(g r_{k-2}\left(K_{3}: F_{10}\right)-1\right)-1>2$, we have $1,2 \notin C(A)$. By the induction hypothesis, we have $|V(G)|=\left|V_{1}\right|+\left|V_{2}\right|+|A|+|B|+|C| \leq$ $\left(g r_{k-1}\left(K_{3}: F_{10}\right)-1\right)+2\left(g r_{k-2}\left(K_{3}: F_{10}\right)-1\right)+1<n$, a contradiction.

Next we suppose $|B|=0$. By Claim 2.1, we have $|A| \geq 1$ and $|C|=1$. If $|A| \geq 2$, then $2 \notin C\left(V_{2}\right)$ and $\left|V_{2}\right| \leq g r_{k-2}\left(K_{3}: F_{10}\right)-1$. Since $|A|=|V(G)|-$ $\left|V_{1}\right|-\left|V_{2}\right|-|C| \geq n-\left(g r_{k-1}\left(K_{3}: F_{10}\right)-1\right)-\left(g r_{k-2}\left(K_{3}: F_{10}\right)-1\right)-1>2$, we have $1,2 \notin C(A)$. Therefore, we have $|V(G)| \leq\left(g r_{k-1}\left(K_{3}: F_{10}\right)-1\right)+$ $2\left(g r_{k-2}\left(K_{3}: F_{10}\right)-1\right)+1<n$, a contradiction. Hence, $|A|=1$. By the minimality of $m$, we have $c(A, C)=2$. By Fact $2.1(1)$, color 2 induces a subgraph $G^{(2)}$ such that each component is a $K_{3}$ or a star within $V_{1}$. In order to avoid a rainbow $K_{3}$ and a monochromatic $P_{4}$ in color 2, there is only a single color between each pair of components. Since $1 \notin C\left(V_{1}\right)$ and by Fact 2.1 (3), $G^{(2)}$ contains at most $n_{k}^{\prime}:=g r_{k-2}\left(K_{3}: K_{3}\right)-1$ components each of which is a $K_{3}$. Moreover, for $0 \leq j \leq n_{k}^{\prime}$, if there are exactly $n_{k}^{\prime}-j$ components each of which is a $K_{3}$, then there are at most $j$ components each of which is 
a nontrivial star. Thus after removing at most $2\left(n_{k}^{\prime}-j\right)+j$ vertices, there is no edge using color 2 within $V_{1}$. Thus $|V(G)|=\left|V_{1}\right|+\left|V_{2}\right|+|A|+|C| \leq$ $2\left|V_{1}\right|+2 \leq 2\left(2\left(n_{k}^{\prime}-j\right)+j+\left(g r_{k-2}\left(K_{3}: F_{10}\right)-1\right)\right)+2<n$, a contradiction.

Case 3. $r=3$.

By Fact 2.1 (3), we may assume that $c\left(V_{1}, V_{2} \cup V_{3}\right)=1$ and $c\left(V_{2}, V_{3}\right)=2$. Thus $1 \notin C\left(V_{1}\right) \cup C\left(V_{2}\right) \cup C\left(V_{3}\right)$ and $2 \notin C\left(V_{2}\right) \cup C\left(V_{3}\right)$. By the induction hypothesis, we have $\left|V_{1}\right| \leq g r_{k-1}\left(K_{3}: F_{10}\right)-1,\left|V_{2}\right| \leq g r_{k-2}\left(K_{3}: F_{10}\right)-1$ and $\left|V_{3}\right| \leq g r_{k-2}\left(K_{3}: F_{10}\right)-1$. Since $m \leq 8$ and $2 \geq\left|V_{4}\right| \geq \cdots \geq\left|V_{m}\right|$, we have $\left|V_{4}\right|+\cdots+\left|V_{m}\right| \leq 10$. If $c\left(v, V_{1}\right)=1$ for some $v \in V_{4} \cup \cdots \cup V_{m}$, then $c\left(v, V_{2}\right) \neq 1$ and $c\left(v, V_{3}\right) \neq 1$ by Fact 2.1 (3). But then $c\left(v, V_{2}\right)=c\left(v, V_{3}\right)=2$, a contradiction. Thus $c\left(V_{1}, V_{4} \cup \cdots \cup V_{m}\right)=2$. Note that $\left|V_{4} \cup \cdots \cup V_{m}\right|=$ $|V(G)|-\left|V_{1}\right|-\left|V_{2}\right|-\left|V_{3}\right| \geq 5$. By Fact 2.1 (2), we have $2 \notin C\left(V_{1}\right)$ and $2 \notin C\left(V_{4} \cup \cdots \cup V_{m}\right)$. Thus $|V(G)|=\left|V_{1}\right|+\left|V_{2}\right|+\left|V_{3}\right|+\left|V_{4} \cup \cdots \cup V_{m}\right| \leq$ $3\left(g r_{k-2}\left(K_{3}: F_{10}\right)-1\right)+\min \left\{g r_{k-1}\left(K_{3}: F_{10}\right)-1,10\right\}<n$, a contradiction.

Case 4. $r \geq 4$.

By Fact 2.1 (3), there is no monochromatic $K_{3}$ in the subgraph $H$ of the reduced graph induced on the first four parts. Thus $H$ is one of the two ${ }^{1}$ 2-edge-colorings of $K_{4}$ without a monochromatic $K_{3}$. By Fact 2.1 (2), $1,2 \notin C\left(V_{1}\right) \cup C\left(V_{2}\right) \cup C\left(V_{3}\right) \cup C\left(V_{4}\right)$. Thus for $i \in[4],\left|V_{i}\right| \leq g r_{k-2}\left(K_{3}:\right.$ $\left.F_{10}\right)-1$, so $\left|V_{5} \cup \cdots \cup V_{m}\right| \geq 5$. If $6 \leq m \leq 8$, then there is a monochromatic $K_{1,2,3}$, a contradiction. Thus $m=5$ and $\left|V_{5}\right| \geq 5$. In order to avoid a monochromatic $F_{10}, G$ is a blow-up of the unique 2-edge-coloring of $K_{5}$ without a monochromatic $K_{3}$. By Fact $2.1(2), 1,2 \notin C\left(V_{1}\right) \cup C\left(V_{2}\right) \cup \cdots \cup C\left(V_{5}\right)$. Applying induction on $k$, we have $|V(G)| \leq 5\left(g r_{k-2}\left(K_{3}: F_{10}\right)-1\right)<n$, a contradiction.

\section{3 (Gallai-)Ramsey numbers for $F_{2, n}$}

We first give our proof of the statement in Theorem 2.2, determining the 2-colored Ramsey numbers for $F_{2, n}$.

\footnotetext{
${ }^{1}$ It is well-known that there are exactly two 2-edge-colorings of $K_{4}$ without a monochromatic copy of $K_{3}$. The first one satisfies that each color induces a $P_{4}$. The second one satisfies that one color induces a $C_{4}$ and the other color induces a $2 K_{2}$.
} 
Proof of Theorem 2.2. Let $n^{\prime}:=2 n-\varepsilon$, where $\varepsilon=1$ if $n$ is even, and $\varepsilon=0$ otherwise. Since $K_{1, n}$ is a subgraph of $F_{2, n}$ and $r_{2}\left(K_{1, n}\right)=n^{\prime}$ (proven in [28]), we have $r_{2}\left(F_{2, n}\right) \geq n^{\prime}$.

For the upper bound, suppose that $G$ is a 2-edge-coloring of $K_{n^{\prime}}$ containing no monochromatic $F_{2, n}$. Since $r_{2}\left(F_{2,3}\right)=6$ (see [97]), we may assume that $n \geq 4$. Then $G$ contains a monochromatic $K_{1, n}$, say $H$ is a monochromatic $K_{1, n}$ in $G$ with $V(H)=\left\{u, v_{1}, v_{2}, \ldots, v_{n}\right\}$ and $c\left(u,\left\{v_{1}, v_{2}, \ldots, v_{n}\right\}\right)=1$. Let $V(G) \backslash V(H)=\left\{w_{1}, w_{2}, \ldots, w_{n^{\prime}-n-1}\right\}$. For each $1 \leq i \leq n^{\prime}-n-1$, there is at most one edge using color 1 in $E\left(w_{i},\left\{v_{1}, v_{2}, \ldots, v_{n}\right\}\right)$ in order to avoid a monochromatic $F_{2, n}$. Note that $n \geq 4$ and $n^{\prime}-n-1 \geq 2$. If $c\left(w_{i},\left\{v_{1}, v_{2}, \ldots\right.\right.$, $\left.\left.v_{n}\right\}\right)=2$ for some $1 \leq i \leq n^{\prime}-n-1$, then it is easy to find a monochromatic $F_{2, n}$ in color 2 , a contradiction. Thus we may consider the following two cases based on $C\left(\left\{w_{1}, w_{2}\right\},\left\{v_{1}, v_{2}, \ldots, v_{n}\right\}\right)$.

For the first case, suppose that $c\left(\left\{w_{1}, w_{2}\right\},\left\{v_{1}, v_{2}, \ldots, v_{n-1}\right\}\right)=2$ and $c\left(\left\{w_{1}, w_{2}\right\}, v_{n}\right)=1$. In order to avoid a monochromatic $F_{2, n}$ in color 2 , we have $c\left(\left\{w_{1}, w_{2}\right\}, u\right)=1$. Then $u w_{1} v_{n} w_{2} u$ is a $C_{4}$ in color 1 , which together with $\left\{v_{1}, v_{2}, \ldots, v_{n-2}\right\}$ forms a monochromatic $F_{2, n}$ centered at $u$, a contradiction. For the second case, suppose that $c\left(\left\{w_{1}, w_{2}\right\},\left\{v_{1}, v_{2}, \ldots, v_{n-2}\right\}\right)=$ $c\left(w_{1} v_{n}\right)=c\left(w_{2} v_{n-1}\right)=2$ and $c\left(w_{1} v_{n-1}\right)=c\left(w_{2} v_{n}\right)=1$. Note that we also have $c\left(\left\{w_{1}, w_{2}\right\}, u\right)=1$, similarly as above. If $c\left(v_{1} v_{n-1}\right)=1$, then $u w_{1} v_{n-1} v_{1} u$ is a $C_{4}$ in color 1 , which together with $\left\{v_{2}, v_{3}, \ldots, v_{n-2}, v_{n}\right\}$ forms a monochromatic $F_{2, n}$ centered at $u$, a contradiction. Thus $c\left(v_{1} v_{n-1}\right)=2$, and by symmetry $c\left(\left\{v_{1}, v_{2}, \ldots, v_{n-2}\right\},\left\{v_{n-1}, v_{n}\right\}\right)=2$. If $n=4$, then there is a $F_{2,4}$ in color 2 centered at $v_{1}$. If $n \geq 5$, then $n^{\prime}-n-1 \geq 4$ and we have $c\left(w_{3} v_{n-1}\right)=2$ or $c\left(w_{3} v_{n}\right)=2$, say $c\left(w_{3} v_{n-1}\right)=2$. Then $v_{n-1} v_{1} v_{n} v_{2} v_{n-1}$ is a $C_{4}$ in color 2 , which together with $\left\{v_{3}, v_{4}, \ldots, v_{n-2}, w_{2}, w_{3}\right\}$ forms a $F_{2, n}$ centered at $v_{n-1}$, a contradiction.

Next, we give our proof of the statement in Theorem 2.3, determining the exact values, lower and upper bounds for $g r_{k}\left(K_{3}: F_{2, n}\right)$.

Proof of Theorem 2.3. We first prove the lower bounds by constructing some appropriate Gallai- $k$-colorings of complete graphs without a monochromatic $F_{2, n}$. For $n \in\{3,4\}$, let $G_{2}$ be a 2-edge-coloring of the complete graph on 
$r_{2}\left(F_{2, n}\right)-1$ vertices using colors 1 and 2 without a monochromatic $F_{2, n}$. We then construct $G_{3}, \ldots, G_{k}$, where for each $3 \leq i \leq k, G_{i}$ is obtained by adding a new vertex to $G_{i-1}$ such that all the new edges get color $i$. Note that $\left|V\left(G_{k}\right)\right|=r_{2}\left(F_{2, n}\right)+k-3$, and $G_{k}$ contains neither a rainbow $K_{3}$ nor a monochromatic $F_{2, n}$.

Let $G^{\prime}$ be the unique 2-edge-coloring of $K_{5}$ using colors 2 and 3 without a monochromatic $K_{3}$. For odd $n \geq 5$, let $H_{1}$ be a monochromatic $K_{(n-1) / 2}$ using color 1 and let $G_{3}$ be the blow-up $G^{\prime}\left(5 \cdot H_{1}\right)$ of $G^{\prime}$. For even $n \geq 6$, let $H_{2}$ (resp., $H_{3}$ ) be a monochromatic $K_{n / 2}$ (resp., $K_{n / 2-1}$ ) using color 1 and let $G_{3}=G^{\prime}\left(1 \cdot H_{2}, 4 \cdot H_{3}\right)$. Let $G_{3}, \ldots, G_{k}$ be a sequence of edge-colorings satisfying that for each $3 \leq i \leq k-1, G_{i+1}$ is obtained by adding a new vertex to $G_{i}$ such that all the new edges get color $i+1$. Note that $G_{k}$ contains neither a rainbow $K_{3}$ nor a monochromatic $F_{2, n}$. For $n \geq 6$, we have

$$
\left|V\left(G_{k}\right)\right|= \begin{cases}5 n / 2+k-7, & \text { if } n \text { is even, } \\ (5 n-1) / 2+k-5, & \text { if } n \text { is odd. }\end{cases}
$$

For $n=5$, we further construct $G_{k}^{\prime}$ by adding a new vertex to $G_{k}$ such that all the new edges get color 1 . Then $G_{k}^{\prime}$ contains neither a rainbow $K_{3}$ nor a monochromatic $F_{2,5}$, and $\left|V\left(G_{k}^{\prime}\right)\right|=k+8$.

For establishing the upper bounds, let

$$
n_{k}:= \begin{cases}r_{2}\left(F_{2, n}\right)+k-2, & \text { if } n \in\{3,4\} \text { and } k \geq 1, \\ k+9, & \text { if } n=5 \text { and } k \geq 2, \\ k(n-1)+2, & \text { if } n \geq 6 \text { and } k \geq 2 .\end{cases}
$$

We will prove that every Gallai- $k$-coloring of $K_{n_{k}}$ contains a monochromatic $F_{2, n}$. The case $k=1$ and $n \in\{3,4\}$ is trivial, and the case $k=2$ and $n \geq 3$ holds by Theorem 2.2. So we may assume $k \geq 3$ in the following. For a contradiction, suppose that $G$ is a Gallai- $k$-coloring of $K_{n_{k}}$ without a monochromatic $F_{2, n}$. We choose such a $G$ where $k$ is minimal. Let $V_{1}, V_{2}, \ldots, V_{m}$ be a Gallai partition with $\left|V_{1}\right| \geq\left|V_{2}\right| \geq \cdots \geq\left|V_{m}\right|$ and $2 \leq m \leq r_{2}\left(F_{2, n}\right)-1$. We choose it such that $m$ is minimal. Assume that colors 1 and 2 are the two colors used between the parts. 
We first suppose $m \geq 4$. In this case, we have $\left|V(G) \backslash V_{1}\right| \geq 3$. In order to avoid a monochromatic $F_{2, n}$ and since $k \geq 3$, we have $2 \leq\left|V_{1}\right| \leq n-1$. For $i \in\{1,2\}$, let $A_{i}$ be the union of parts with color $i$ to $V_{1}$. If $n \notin\{4,5\}$, then $\max \left\{\left|A_{1}\right|,\left|A_{2}\right|\right\} \geq\left\lceil\left(|V(G)|-\left|V_{1}\right|\right) / 2\right\rceil \geq\left\lceil\left(n_{k}-(n-1)\right) / 2\right\rceil \geq n$, which implies that there is a monochromatic $F_{2, n}$, a contradiction. If $n=5$, then we must have $\left|V_{1}\right|=\left|A_{1}\right|=\left|A_{2}\right|=4$ and $k=3$ by a similar argument. For any edge $u v \in E\left(A_{1}, A_{2}\right)$, we have $c(u v) \in\{1,2\}$, resulting in a monochromatic $F_{2,5}$, a contradiction. If $n=4$, then $2 \leq\left|V_{1}\right| \leq 3$ and $3 \geq \max \left\{\left|A_{1}\right|,\left|A_{2}\right|\right\} \geq$ $\lceil(k+5-3) / 2\rceil \geq 3$, say $\left|A_{1}\right|=3$. If $\left|V_{1}\right|=3$, then $2 \leq\left|A_{2}\right| \leq 3$. For avoiding a monochromatic $F_{2,4}$, we have $1,2 \notin C\left(A_{1}, A_{2}\right)$, a contradiction. If $\left|V_{1}\right|=2$, then $\left|A_{2}\right|=3$ and $k=3$. Let $A_{1}=\left\{u_{1}, u_{2}, u_{3}\right\}$ and $A_{2}=\left\{v_{1}, v_{2}, v_{3}\right\}$. In order to avoid a monochromatic $F_{2,4}$, there is at most one edge using color 1 between $u_{i}$ and $A_{2}$ for each $i \in[3]$, and there is at most one edge using color 2 between $v_{j}$ and $A_{1}$ for each $j \in[3]$. Thus there are at most six edges between $A_{1}$ and $A_{2}$, a contradiction.

Therefore, we have $2 \leq m \leq 3$. In this case, we may assume that $m=2$, since every Gallai-coloring admitting a Gallai partition with exactly three parts also admits a Gallai partition with exactly two parts. Without loss of generality, let $c\left(V_{1}, V_{2}\right)=1$. Since $k \geq 3$, we have $\left|V_{1}\right| \geq\lceil|V(G)| / 2\rceil \geq n$. Thus $\left|V_{2}\right|=1$ for avoiding a monochromatic $F_{2, n}$ in color 1 . If $1 \notin C\left(V_{1}\right)$, then $|V(G)|=\left|V_{1}\right|+\left|V_{2}\right| \leq\left(n_{k-1}-1\right)+1<n_{k}$ by the minimality of $k$, a contradiction. Thus $1 \in C\left(V_{1}\right)$, say $v_{1}, v_{2} \in V_{1}$ with $c\left(v_{1} v_{2}\right)=1$. In order to avoid a monochromatic $F_{2, n}$ in color 1 , we have $1 \notin C\left(\left\{v_{1}, v_{2}\right\}, V_{1} \backslash\left\{v_{1}, v_{2}\right\}\right)$. Since $G$ is rainbow $K_{3}$-free, we have $c\left(v_{1} v\right)=c\left(v_{2} v\right)$ for any $v \in V_{1} \backslash\left\{v_{1}, v_{2}\right\}$. In order to avoid a monochromatic $F_{2, n}$, there are at most $n-1$ vertices in $V_{1} \backslash\left\{v_{1}, v_{2}\right\}$ with a single color to $\left\{v_{1}, v_{2}\right\}$. Note that $\left|V_{1} \backslash\left\{v_{1}, v_{2}\right\}\right|=n_{k}-3$ and $\left|C\left(\left\{v_{1}, v_{2}\right\}, V_{1} \backslash\left\{v_{1}, v_{2}\right\}\right)\right| \leq k-1$.

If $n \geq 6$, then there are at least $\left\lceil\left(n_{k}-3\right) /(k-1)\right\rceil \geq n$ vertices in $V_{1} \backslash\left\{v_{1}, v_{2}\right\}$ with a single color to $\left\{v_{1}, v_{2}\right\}$. This implies that there is a monochromatic $F_{2, n}$, a contradiction.

If $n=3$, then there exist four vertices $u_{1}, u_{2}, u_{3}, u_{4} \in V_{1} \backslash\left\{v_{1}, v_{2}\right\}$ such that $c\left(\left\{v_{1}, v_{2}\right\},\left\{u_{1}, u_{2}\right\}\right)=i$ and $c\left(\left\{v_{1}, v_{2}\right\},\left\{u_{3}, u_{4}\right\}\right)=j$, where $2 \leq i<j \leq k$. Since $G$ is rainbow $K_{3}$-free, we have $C\left(\left\{u_{1}, u_{2}\right\},\left\{u_{3}, u_{4}\right\}\right) \subseteq\{i, j\}$. Then there is a monochromatic $F_{2,3}$ in color $i$ or $j$, a contradiction. 
If $n=4$, then we may consider the following two cases. Firstly, suppose there exist five vertices $u_{1}, u_{2}, \ldots, u_{5} \in V_{1} \backslash\left\{v_{1}, v_{2}\right\}$ such that $c\left(\left\{v_{1}, v_{2}\right\}\right.$, $\left.\left\{u_{1}, u_{2}, u_{3}\right\}\right)=i$ and $c\left(\left\{v_{1}, v_{2}\right\},\left\{u_{4}, u_{5}\right\}\right)=j$, where $i$ and $j$ are two distinct colors in $\{2,3, \ldots, k\}$. We have $C\left(\left\{u_{1}, u_{2}, u_{3}\right\},\left\{u_{4}, u_{5}\right\}\right) \subseteq\{i, j\}$ since $G$ is rainbow $K_{3}$-free. In order to avoid a monochromatic $F_{2,4}$ in color $j$, there are at least two edges using color $i$ between $u_{4}$ (resp., $u_{5}$ ) and $\left\{u_{1}, u_{2}, u_{3}\right\}$. Thus there exists a vertex, say $u_{1}$, such that $c\left(u_{1},\left\{u_{4}, u_{5}\right\}\right)=i$, resulting in a monochromatic $F_{2,4}$, a contradiction.

Secondly, suppose there exist six vertices $u_{1}, u_{2}, \ldots, u_{6} \in V_{1} \backslash\left\{v_{1}, v_{2}\right\}$ with $c\left(\left\{v_{1}, v_{2}\right\},\left\{u_{1}, u_{2}\right\}\right)=i, c\left(\left\{v_{1}, v_{2}\right\},\left\{u_{3}, u_{4}\right\}\right)=j$ and $c\left(\left\{v_{1}, v_{2}\right\},\left\{u_{5}, u_{6}\right\}\right)=\ell$, where $2 \leq i<j<\ell \leq k$. Without loss of generality, we may assume that $c\left(u_{1} u_{3}\right)=i$. Then $c\left(u_{1} u_{4}\right)=j$ and $c\left(u_{1},\left\{u_{5}, u_{6}\right\}\right)=\ell$. Since $c\left(u_{4} u_{5}\right) \in\{j, \ell\}$, there is a monochromatic $F_{2,4}$ in color $j$ or $\ell$, a contradiction.

If $n=5$, then by the Pigeonhole Principle, we may consider the following two cases. Firstly, suppose there exist nine vertices $u_{1}, u_{2}, \ldots, u_{9} \in V_{1} \backslash\left\{v_{1}, v_{2}\right\}$ with $c\left(\left\{v_{1}, v_{2}\right\},\left\{u_{1}, u_{2}, u_{3}, u_{4}\right\}\right)=i, c\left(\left\{v_{1}, v_{2}\right\},\left\{u_{5}, u_{6}, u_{7}\right\}\right)=j$ and $c\left(\left\{v_{1}, v_{2}\right\}\right.$, $\left.\left\{u_{8}, u_{9}\right\}\right)=\ell$, where $i, j$ and $\ell$ are three distinct colors in $\{2,3, \ldots, k\}$. In order to avoid a monochromatic $F_{2,5}$ in color $j$ (resp., $\ell$ ), there are at most two edges using color $j$ (resp., $\ell$ ) between each $u \in\left\{u_{5}, u_{6}, u_{7}\right\}$ (resp., $\left\{u_{8}, u_{9}\right\}$ ) and $\left\{u_{1}, u_{2}, u_{3}, u_{4}\right\}$. Hence, there are at least ten edges using color $i$ between $\left\{u_{1}, u_{2}, u_{3}, u_{4}\right\}$ and $\left\{u_{5}, u_{6}, \ldots, u_{9}\right\}$. In particular, there exists a vertex $u \in$ $\left\{u_{1}, u_{2}, u_{3}, u_{4}\right\}$ such that there are at least three edges using color $i$ between $u$ and $\left\{u_{5}, u_{6}, \ldots, u_{9}\right\}$, resulting in a monochromatic $F_{2,5}$, a contradiction.

Secondly, suppose there exist eight vertices $u_{1}, u_{2}, \ldots, u_{8} \in V_{1} \backslash\left\{v_{1}, v_{2}\right\}$ with $c\left(\left\{v_{1}, v_{2}\right\},\left\{u_{1}, u_{2}\right\}\right)=i, c\left(\left\{v_{1}, v_{2}\right\},\left\{u_{3}, u_{4}\right\}\right)=j, c\left(\left\{v_{1}, v_{2}\right\},\left\{u_{5}, u_{6}\right\}\right)=s$ and $c\left(\left\{v_{1}, v_{2}\right\},\left\{u_{7}, u_{8}\right\}\right)=t$, where $2 \leq i<j<s<t \leq k$. Since there are at most two edges using color $i$ in $E\left(u_{1},\left\{u_{3}, u_{4}, \ldots, u_{8}\right\}\right)$, we may assume that $c\left(u_{1},\left\{u_{7}, u_{8}\right\}\right)=t$ without loss of generality. Then there is at most one edge using color $t$ in $E\left(u_{7},\left\{u_{3}, u_{4}, u_{5}, u_{6}\right\}\right)$, so we may assume that $c\left(u_{7},\left\{u_{5}, u_{6}\right\}\right)=$ $s$. For avoiding a rainbow $K_{3}$, we have $c\left(u_{1},\left\{u_{5}, u_{6}\right\}\right)=s$. For avoiding a monochromatic $F_{2,5}$ in color $s$, we have $c\left(u_{8},\left\{u_{5}, u_{6}\right\}\right)=t$, resulting in a monochromatic $F_{2,5}$ in color $t$ with center $u_{8}$, a contradiction. 


\subsection{Gallai-Ramsey numbers for $F_{12}$ and $F_{13}$}

In this section, we will prove Theorem 2.4. We start by recalling the following known Ramsey numbers which will be used in our proofs.

Theorem 2.10 (Hendry [97]). $r_{2}\left(F_{12}\right)=r_{2}\left(F_{13}\right)=10$.

Theorem 2.4 follows immediately from the following two lemmas, the first of which provides lower bounds for $g r_{k}\left(K_{3}: F_{12}\right)$ and $g r_{k}\left(K_{3}: F_{13}\right)$, and the second of which provides the matching upper bounds for $g r_{k}\left(K_{3}: F_{12}\right)$ and $g r_{k}\left(K_{3}: F_{13}\right)$.

Lemma 2.11. For any integer $k \geq 1$ and graph $H \in\left\{F_{12}, F_{13}\right\}$, we have

$$
g r_{k}\left(K_{3}: H\right)> \begin{cases}9 \cdot 5^{(k-2) / 2}, & \text { if } k \text { is even, } \\ 4 \cdot 5^{(k-1) / 2}, & \text { if } k \text { is odd }\end{cases}
$$

Proof. If $k$ is even, then let $G_{2}$ be a 2-edge-colored $K_{9}$ which contains no monochromatic $H$, using colors 1 and 2 . Suppose that $2 i<k$ and we have constructed a (2i)-edge-coloring $G_{2 i}$ of $K_{n_{2 i}}$ containing neither a rainbow $K_{3}$ nor a monochromatic $H$, where $n_{2 i}:=9 \cdot 5^{(2 i-2) / 2}$. Let $G^{\prime}$ be a 2-edge-colored $K_{5}$ using colors $2 i+1$ and $2 i+2$ which contains no monochromatic $K_{3}$, i.e., colors $2 i+1$ and $2 i+2$ induce two monochromatic copies of $C_{5}$. We construct $G_{2 i+2}$ such that $G_{2 i+2}=G^{\prime}\left(5 \cdot G_{2 i}\right)$ is a blow-up of $G^{\prime}$. Finally, we obtain a $k$ edge-coloring $G_{k}$ of $K_{n}$ containing neither a rainbow $K_{3}$ nor a monochromatic $H$, where $n=9 \cdot 5^{(k-2) / 2}$.

If $k$ is odd, then let $G_{1}$ be a monochromatic $K_{4}$ using color 1 . Suppose that $2 i-1<k$ and we have constructed a $(2 i-1)$-edge-coloring $G_{2 i-1}$ of $K_{n_{2 i-1}}$ which contains neither a rainbow $K_{3}$ nor a monochromatic $H$, where $n_{2 i-1}:=4 \cdot 5^{(2 i-2) / 2}$. Let $G^{\prime \prime}$ be a 2-edge-colored $K_{5}$ using colors $2 i$ and $2 i+1$ which contains no monochromatic $K_{3}$. We construct $G_{2 i+1}$ such that $G_{2 i+1}=G^{\prime \prime}\left(5 \cdot G_{2 i-1}\right)$ is a blow-up of $G^{\prime \prime}$. Finally, we obtain a $k$-edge-coloring $G_{k}$ of $K_{n}$ containing neither a rainbow $K_{3}$ nor a monochromatic $H$, where $n=4 \cdot 5^{(k-1) / 2}$. 
Lemma 2.12. For any integer $k \geq 1$ and graph $H \in\left\{F_{12}, F_{13}\right\}$, we have

$$
g r_{k}\left(K_{3}: H\right) \leq \begin{cases}9 \cdot 5^{(k-2) / 2}+1, & \text { if } k \text { is even }, \\ 4 \cdot 5^{(k-1) / 2}+1, & \text { if } k \text { is odd }\end{cases}
$$

Proof. We prove the statement by induction on $k$. The case $k=1$ is trivial and the case $k=2$ holds by Theorem 2.10 , so we may assume $k \geq 3$. Suppose the statement holds for all $k^{\prime}<k$, and we will prove it for $k$. For a contradiction, suppose that $G$ is a Gallai- $k$-coloring of $K_{n}$ containing no monochromatic $H$, where

$$
n:= \begin{cases}9 \cdot 5^{(k-2) / 2}+1, & \text { if } k \text { is even, } \\ 4 \cdot 5^{(k-1) / 2}+1, & \text { if } k \text { is odd. }\end{cases}
$$

Since $k \geq 3$, we have $n \geq 21$. Let $V_{1}, V_{2}, \ldots, V_{m}$ be a Gallai partition with $\left|V_{1}\right| \geq\left|V_{2}\right| \geq \cdots \geq\left|V_{m}\right|$ and $2 \leq m \leq r_{2}(H)-1=9$. We choose it such that $m$ is minimal. We may assume that colors 1 and 2 are the two colors used between the parts (if there is only one color used between the parts, then let color 1 be this color). We first have the following simple facts since $G$ is monochromatic $H$-free.

Fact 2.2. Let $U, V$ be two nonempty subsets of $V(G)$ with $U \cap V=\emptyset$ and $c(U, V)=c_{0}$, where $c_{0} \in[k]$. Then

(1) there is no monochromatic $P_{4}$ using color $c_{0}$ within $U$ or $V$, respectively;

(2) if $|U| \geq 3$ and $|V| \geq 3$, then $c_{0} \notin C(U)$ and $c_{0} \notin C(V)$, respectively;

(3) if $|U| \geq 3$ and $|V| \geq 2$, then there is no monochromatic $P_{3}$ using color $c_{0}$ within $U$;

(4) if $|U| \geq 4$, then there is no monochromatic $K_{3}$ using color $c_{0}$ within $U$;

(5) if $|U| \geq 2$ and $|V| \geq 2$, then there is no vertex with color $c_{0}$ to both $U$ and $V$ in $V(G) \backslash(U \cup V)$.

Fact 2.3. Let $U, V$ be two nonempty subsets of $V(G)$ with $U \cap V=\emptyset$ and $c(U, V)=c_{0}$, where $c_{0} \in[k]$. Then 
(1) if $H=F_{12}$ and $|U| \geq 4$, then there is no monochromatic $P_{3}$ using color $c_{0}$ within $U$;

(2) if $H=F_{13},|U| \geq 3$ and $|V| \geq 2$, then $c_{0} \notin C(U)$.

Claim 2.2. We have $4 \leq m \leq 9$, and $C\left(V_{i}, \bigcup_{j \in[m] \backslash\{i\}} V_{j}\right)=\{1,2\}$ for every $i \in[m]$.

Proof. If $m=3$, then at least two of the colors $c\left(V_{1}, V_{2}\right), c\left(V_{1}, V_{3}\right)$ and $c\left(V_{2}, V_{3}\right)$ are the same color, say $c\left(V_{1}, V_{2}\right)=c\left(V_{1}, V_{3}\right)$. This implies that $V_{1}$ and $V(G) \backslash V_{1}$ form a Gallai partition with exactly two parts, contradicting the minimality of $m$. If $m=2$, then $c\left(V_{1}, V_{2}\right)=1$. If $\left|V_{2}\right| \geq 3$, then $1 \notin C\left(V_{1}\right)$ by Fact 2.2 (2). Applying induction on $k$ within $V_{1}$, we have $|V(G)|=\left|V_{1}\right|+\left|V_{2}\right| \leq 2\left|V_{1}\right| \leq$ $2\left(g r_{k-1}\left(K_{3}: H\right)-1\right)<n$, a contradiction. If $\left|V_{2}\right|=2$, then by Fact 2.2 (3), color 1 induces a matching within $V_{1}$. If $\left|V_{2}\right|=1$, then by Fact 2.2 (1) and (4), color 1 induces a subgraph such that each component is a star within $V_{1}$. Thus when $1 \leq\left|V_{2}\right| \leq 2$, we can partition $V_{1}$ into two subsets $V_{1}^{\prime}$ and $V_{1}^{\prime \prime}$ such that $1 \notin C\left(V_{1}^{\prime}\right)$ and $1 \notin C\left(V_{1}^{\prime \prime}\right)$. By the induction hypothesis, $|V(G)|=\left|V_{1}^{\prime}\right|+\left|V_{1}^{\prime \prime}\right|+\left|V_{2}\right| \leq 2\left(g r_{k-1}\left(K_{3}: H\right)-1\right)+2<n$, a contradiction. Therefore, $4 \leq m \leq 9$.

If $\left|C\left(V_{i}, \bigcup_{j \in[m] \backslash\{i\}} V_{j}\right)\right|=1$ for some $i \in[m]$, then $V_{i}$ and $V(G) \backslash V_{i}$ form a Gallai partition with exactly two parts, contradicting the minimality of $m$.

By Claim 2.2, we have $4 \leq m \leq 9$. Let $r$ be the number of parts with at least three vertices, i.e., $\left|V_{r}\right| \geq 3$ and $\left|V_{r+1}\right| \leq 2$ (here we define $\left|V_{m+1}\right|=0$ ). Since $m \leq 9$ and $|V(G)| \geq 21$, we have $1 \leq r \leq m \leq 9$. We divide the rest of the proof into four cases based on the number $r$.

Case 1. $r \geq 4$.

By Fact 2.2 (5), there is no monochromatic $K_{3}$ in the subgraph $R$ of the reduced graph induced on the first four parts. Thus $R$ is one of the two 2-edge-colorings of $K_{4}$ without a monochromatic $K_{3}$. Thus $1,2 \notin C\left(V_{1}\right)$ by Fact 2.2 (2). If $m \leq 5$, then $|V(G)| \leq 5\left|V_{1}\right| \leq 5\left(g r_{k-2}\left(K_{3}: H\right)-1\right)<n$, a contradiction. If $6 \leq m \leq 9$, then $1 \geq\left|V_{5}\right| \geq \cdots \geq\left|V_{m}\right|$; otherwise there is a monochromatic $K_{1,2,3}$ since $r_{2}\left(K_{3}\right)=6$, a contradiction. Moreover, since $n=|V(G)|=\sum_{i=1}^{4}\left|V_{i}\right|+\sum_{j=5}^{m}\left|V_{j}\right| \leq 4\left|V_{1}\right|+5 \leq 4\left(g r_{k-2}\left(K_{3}: H\right)-1\right)+5$, 
we have $n=21, k=3,\left|V_{i}\right|=4$ for $i \in[4]$ and $\left|V_{j}\right|=1$ for $j \in\{5,6, \ldots, 9\}$. Note that there is a monochromatic $K_{3}$ in the subgraph of the reduced graph induced on $V_{1}, V_{2}, \ldots, V_{6}$, i.e., there is a monochromatic $K_{1,4,4}$ or $K_{1,1,4}$ in $G$. If the former holds, then we are done. If the latter holds, then there is a monochromatic $F_{12}$ using six edges of the $K_{1,1,4}$, and a monochromatic $F_{13}$ using five edges of the $K_{1,1,4}$ and one edge between the first four parts, a contradiction.

Case 2. $r=3$.

In order to avoid a monochromatic $K_{3,3,3}$ which contains a monochromatic $H$, we may assume that $c\left(V_{1}, V_{2} \cup V_{3}\right)=1$ and $c\left(V_{2}, V_{3}\right)=2$. Then $1 \notin C\left(V_{1}\right)$ and $1,2 \notin C\left(V_{2}\right) \cup C\left(V_{3}\right)$ by Fact $2.2(2)$. By the induction hypothesis, we have $\left|V_{1}\right| \leq g r_{k-1}\left(K_{3}: H\right)-1,\left|V_{2}\right| \leq g r_{k-2}\left(K_{3}: H\right)-1$ and $\left|V_{3}\right| \leq g r_{k-2}\left(K_{3}: H\right)-1$. So $\left|V_{4} \cup \cdots \cup V_{m}\right| \geq 4$. Recall that $m \leq 9$ and $2 \geq\left|V_{4}\right| \geq \cdots \geq\left|V_{m}\right|$. Thus $\left|V_{4} \cup \cdots \cup V_{m}\right| \leq 2+2+1+1+1+1=8$; otherwise there is a monochromatic $K_{1,2,2}$ in $G$. If $c\left(v, V_{1}\right)=1$ for some $v \in V_{4} \cup \cdots \cup V_{m}$, then $c\left(v, V_{2}\right) \neq 1$ and $c\left(v, V_{3}\right) \neq 1$ by Fact $2.2(5)$. But then $c\left(v, V_{2}\right)=c\left(v, V_{3}\right)=2$, a contradiction. Thus $c\left(V_{1}, V_{4} \cup \cdots \cup V_{m}\right)=2$. Recall that we have $\left|V_{4} \cup \cdots \cup V_{m}\right| \geq 4$. Thus $2 \notin C\left(V_{1}\right)$ and $2 \notin C\left(V_{4} \cup \cdots \cup V_{m}\right)$ by Fact $2.2(2)$. Hence, $\left|V_{1}\right| \leq g r_{k-2}\left(K_{3}\right.$ : $H)-1$ and $\left|V_{4} \cup \cdots \cup V_{m}\right| \leq \min \left\{8, g r_{k-1}\left(K_{3}: H\right)-1\right\}=8$. Then $|V(G)|=$ $\sum_{i=1}^{3}\left|V_{i}\right|+\sum_{j=4}^{m}\left|V_{j}\right| \leq 3\left(g r_{k-2}\left(K_{3}: H\right)-1\right)+8<n$, a contradiction.

Case 3. $r=1$.

For each $i \in\{1,2\}$, let $A_{i}$ be the union of parts with color $i$ to $V_{1}$. Then $\left|A_{1}\right|+\left|A_{2}\right| \leq 16$. If $\left|A_{1}\right| \geq 3$ and $\left|A_{2}\right| \geq 3$, then $1,2 \notin C\left(V_{1}\right)$ by Fact 2.2 (2). By the induction hypothesis, we have $|V(G)|=\left|V_{1}\right|+\left|A_{1}\right|+\left|A_{2}\right| \leq$ $g r_{k-2}\left(K_{3}: H\right)-1+16<n$, a contradiction. If $\left|A_{1}\right| \geq 3$ and $\left|A_{2}\right| \leq 2$, then $1 \notin C\left(V_{1}\right)$ and $1 \notin C\left(A_{1}\right)$ by Fact 2.2 (2). By the induction hypothesis, $|V(G)|=\left|V_{1}\right|+\left|A_{1}\right|+\left|A_{2}\right| \leq 2\left(g r_{k-1}\left(K_{3}: H\right)-1\right)+2<n$, a contradiction. Thus $\left|A_{1}\right| \leq 2$, and by symmetry $\left|A_{2}\right| \leq 2$. By Claim 2.2 , we have $\left|A_{1}\right| \geq 1,\left|A_{2}\right| \geq 1$ and $\max \left\{\left|A_{1}\right|,\left|A_{2}\right|\right\}=2$, say $1 \leq\left|A_{2}\right| \leq\left|A_{1}\right|=2$. Note that $\left|V_{1}\right| \geq n-4 \geq 17$.

If $H=F_{12}$, then there is no monochromatic $P_{3}$ using color 1 or color 2 within $V_{1}$ by Fact 2.3 (1). Thus there is no rainbow $P_{3}$ using colors 1 and 2; otherwise there is a monochromatic $P_{3}$ using color 1 or 2 since $G$ is rainbow $K_{3}$-free. Thus colors 1 and 2 induce a matching within $V_{1}$. Then there exists a 
subset $U \subseteq V_{1}$ with $|U| \leq\left\lfloor\left|V_{1}\right| / 2\right\rfloor$ such that $1,2 \notin C\left(V_{1} \backslash U\right)$. By the induction hypothesis, $|V(G)|=\left|V_{1}\right|+\left|A_{1}\right|+\left|A_{2}\right| \leq 2\left(g r_{k-2}\left(K_{3}: F_{12}\right)-1\right)+4<n$, a contradiction.

If $H=F_{13}$, then $1 \notin C\left(V_{1}\right)$ by Fact 2.3 (2). Now $|V(G)|=\left|V_{1}\right|+\left|A_{1}\right|+\left|A_{2}\right| \leq$ $g r_{k-1}\left(K_{3}: F_{13}\right)-1+4<n$, a contradiction.

Case 4. $r=2$.

In this case, we may assume that $c\left(V_{1}, V_{2}\right)=1$. Then by Fact 2.2 (2), we have $1 \notin C\left(V_{i}\right)$ for $i \in\{1,2\}$. Thus $\left|V_{i}\right| \leq g r_{k-1}\left(K_{3}: H\right)-1$. Recall that $m \leq 9$ and $2 \geq\left|V_{3}\right| \geq \cdots \geq\left|V_{m}\right|$. If $\left|V_{6}\right| \geq 2$, then there is a monochromatic $K_{2,2,2}$ in $G$, contradicting Fact $2.2(5)$. Hence, $\left|V_{3} \cup \cdots \cup V_{m}\right| \leq 2+2+2+(m-5) \leq 10$. By Fact 2.2 (5), there is no vertex with color 1 to both $V_{1}$ and $V_{2}$. Thus we can partition $V_{3} \cup \cdots \cup V_{m}$ into three parts $A, B$ and $C$ such that $c\left(A, V_{1}\right)=$ $c\left(C, V_{2}\right)=1$ and $c\left(A, V_{2}\right)=c\left(C, V_{1}\right)=c\left(B, V_{1} \cup V_{2}\right)=2$. Note that we have $|A \cup B| \leq 2$ or $|B \cup C| \leq 2$; otherwise $2 \notin C\left(V_{1}\right)$ and $2 \notin C\left(V_{2}\right)$ by Fact 2.2 (2), and then $|V(G)|=\left|V_{1}\right|+\left|V_{2}\right|+|A|+|B|+|C| \leq 2\left(g r_{k-2}\left(K_{3}: H\right)-1\right)+10<n$, a contradiction. Without loss of generality, we may assume $|B \cup C| \leq 2$, so $|B| \leq 2$ and $|C| \leq 2$.

If $H=F_{12}$, then we first suppose $|B|=2$ and $|C|=0$. By the minimality of $m$, we have $|A| \neq 0$, and thus $2 \notin C\left(V_{2}\right)$. If $|A| \geq 3$, then $1,2 \notin C(A)$. Thus $|V(G)| \leq\left(g r_{k-1}\left(K_{3}: F_{12}\right)-1\right)+2\left(g r_{k-2}\left(K_{3}: F_{12}\right)-1\right)+2<n$, a contradiction. Hence, $|A| \leq 2$, but then $|V(G)| \leq\left(g r_{k-1}\left(K_{3}: F_{12}\right)-1\right)+\left(g r_{k-2}\left(K_{3}: F_{12}\right)-\right.$ 1) $+4<n$, a contradiction.

Next, we suppose $|B|=1$ and $|C| \leq 1$. If $|A| \geq 3$ or $|A|=2$, then we can derive a contradiction similarly as above. Thus $|A| \leq 1$. If $|A|=0$ or $|C|=0$, then $|V(G)| \leq 2\left(g r_{k-1}\left(K_{3}: F_{12}\right)-1\right)+2<n$. Hence, $|A|=|C|=1$. By Fact 2.2 (3), color 2 induces a matching within $V_{1}$ and $V_{2}$. For each $i \in\{1,2\}$, we can delete all the edges of color 2 within $V_{i}$ after removing at most $\left\lfloor\left|V_{i}\right| / 2\right\rfloor$ vertices. Thus $|V(G)| \leq 4\left(g r_{k-2}\left(K_{3}: F_{12}\right)-1\right)+3<n$, a contradiction.

Hence, we have $|B|=0$ and $|C| \leq 2$. If we further have $|A| \geq 3$, then $2 \notin C\left(V_{2}\right)$ and $1,2 \notin C(A)$. This implies $|V(G)| \leq\left(g r_{k-1}\left(K_{3}: F_{12}\right)-1\right)+$ $2\left(g r_{k-2}\left(K_{3}: F_{12}\right)-1\right)+2<n$, a contradiction. Thus $|A| \leq 2$. Note that $\left|V_{2}\right| \geq 4$; otherwise $|V(G)| \leq\left(g r_{k-1}\left(K_{3}: F_{12}\right)-1\right)+3+4<n$. By the minimality of $m$, we have $|A| \neq 0$ and $|C| \neq 0$. By Fact 2.3 (1), color 2 induces 
a matching within $V_{1}$ and $V_{2}$. Thus $|V(G)| \leq 4\left(g r_{k-2}\left(K_{3}: F_{12}\right)-1\right)+4<n$, a contradiction.

If $H=F_{13}$, then $|A \cup B| \leq 1$ or $|B \cup C| \leq 1$; otherwise $2 \notin C\left(V_{1}\right)$ and $2 \notin C\left(V_{2}\right)$ by Fact $2.3(2)$, and then $|V(G)|=\left|V_{1}\right|+\left|V_{2}\right|+|A|+|B|+|C| \leq$ $2\left(g r_{k-2}\left(K_{3}: F_{13}\right)-1\right)+10<n$, a contradiction. Without loss of generality, we may assume $|B \cup C| \leq 1$, so $|B| \leq 1$ and $|C| \leq 1$. We first suppose $|B|=1$ and $|C|=0$. By the minimality of $m$, we have $|A| \neq 0$. Then $2 \notin C\left(V_{2}\right)$ by Fact 2.3 (2), so $|A| \geq n-\left(g r_{k-1}\left(K_{3}: F_{13}\right)-1\right)-\left(g r_{k-2}\left(K_{3}: F_{13}\right)-1\right)-1>3$. Then $1,2 \notin C(A)$, so $|V(G)|=\left|V_{1}\right|+\left|V_{2}\right|+|A|+1 \leq\left(g r_{k-1}\left(K_{3}: F_{13}\right)-1\right)+$ $2\left(g r_{k-2}\left(K_{3}: F_{13}\right)-1\right)+1<n$, a contradiction.

Therefore, $|B|=0$ and $|C| \leq 1$. By the minimality of $m$, we have $|A| \geq 1$ and $|C|=1$. If $|A| \geq 3$, then $2 \notin C\left(V_{2}\right)$ and $1,2 \notin C(A)$. Thus $|V(G)| \leq$ $\left(g r_{k-1}\left(K_{3}: F_{13}\right)-1\right)+2\left(g r_{k-2}\left(K_{3}: F_{13}\right)-1\right)+1<n$, a contradiction. If $|A|=2$, then $2 \notin C\left(V_{2}\right)$, which implies $|V(G)| \leq\left(g r_{k-1}\left(K_{3}: F_{13}\right)-1\right)+$ $\left(g r_{k-2}\left(K_{3}: F_{13}\right)-1\right)+3<n$, a contradiction. Hence $|A|=|C|=1$. Then $|V(G)| \leq 2\left(g r_{k-1}\left(K_{3}: F_{13}\right)-1\right)+2<n$, a contradiction. 


\section{Chapter 3}

\section{The Gallai-Ramsey number for $K_{4}+e$}

In this chapter, we determine the Gallai-Ramsey number $g r_{k}\left(K_{3}: K_{4}+e\right)$ for the graph $K_{4}+e$ on five vertices consisting of a $K_{4}$ with a pendant edge. At the end of the chapter, we also give a short survey on known Gallai-Ramsey numbers and some open problems.

\subsection{Introduction}

Recall that the Gallai-Ramsey number $g r_{k}\left(K_{3}: H\right)$ is the minimum integer $n$ such that every $k$-edge-coloring of $K_{n}$ contains either a rainbow copy of $K_{3}$ or a monochromatic copy of $H$. Let $K_{4}+e$ denote the graph on five vertices consisting of a $K_{4}$ with a pendant edge, i.e., $K_{4}+e$ is the graph obtained from the graph $F_{12}$ of Figure 2.1 by joining the two vertices with degree 2 by an edge. We prove the following result.

Theorem 3.1. For any integer $k \geq 1$, we have

$$
g r_{k}\left(K_{3}: K_{4}+e\right)= \begin{cases}17^{k / 2}+1, & \text { if } k \text { is even } \\ 4 \cdot 17^{(k-1) / 2}+1, & \text { if } k \text { is odd }\end{cases}
$$


In fact, we will prove a more general result. For an integer $s$ with $0 \leq$ $s \leq k$, if $H_{1}=\cdots=H_{s}=K_{4}+e$ and $H_{s+1}=\cdots=H_{k}=K_{3}$, we will write $g r_{k}\left(K_{3}: s \cdot K_{4}+e,(k-s) \cdot K_{3}\right)$ for $g r\left(K_{3}: H_{1}, H_{2}, \ldots, H_{k}\right)$. In this chapter, we will prove Theorem 3.1 in the following more general form. Theorem 3.1 immediately follows from Theorem 3.2 by choosing $s=k$.

Theorem 3.2. For integers $k \geq 1$ and $0 \leq s \leq k$, we have

$$
\begin{aligned}
& g r_{k}\left(K_{3}: s \cdot K_{4}+e,(k-s) \cdot K_{3}\right) \\
& = \begin{cases}17^{s / 2} \cdot 5^{(k-s) / 2}+1, & \text { if } s \text { is even and } k-s \text { is even, } \\
2 \cdot 17^{s / 2} \cdot 5^{(k-s-1) / 2}+1, & \text { if } s \text { is even and } k-s \text { is odd, } \\
8 \cdot 17^{(s-1) / 2} \cdot 5^{(k-s-1) / 2}+1, & \text { if } s \text { is odd and } k-s \text { is odd, } \\
4 \cdot 17^{(s-1) / 2} \cdot 5^{(k-s) / 2}+1, & \text { if } s \text { is odd and } k-s \text { is even. }\end{cases}
\end{aligned}
$$

We will prove the above result in the usual way, by establishing suitable lower bounds for the four cases in Section 3.2, and matching upper bounds in Section 3.3. We will need the following known Ramsey numbers in our proofs.

Theorem 3.3. The following Ramsey numbers have been established:

(1) (Greenwood and Gleason [88]) $r\left(K_{3}, K_{3}\right)=6, r\left(K_{4}, K_{4}\right)=18$;

(2) $($ Clancy $[41]) r\left(K_{4}+e, K_{3}\right)=9$;

(3) (Harborth and Mengersen [95]) $r\left(K_{4}+e, K_{4}+e\right)=18$.

\subsection{Proof of the lower bounds}

For convenience, let

$$
g(k, s):= \begin{cases}17^{s / 2} \cdot 5^{(k-s) / 2}, & \text { if } s \text { is even and } k-s \text { is even, } \\ 2 \cdot 17^{s / 2} \cdot 5^{(k-s-1) / 2}, & \text { if } s \text { is even and } k-s \text { is odd, } \\ 8 \cdot 17^{(s-1) / 2} \cdot 5^{(k-s-1) / 2}, & \text { if } s \text { is odd and } k-s \text { is odd, } \\ 4 \cdot 17^{(s-1) / 2} \cdot 5^{(k-s) / 2}, & \text { if } s \text { is odd and } k-s \text { is even. }\end{cases}
$$


We shall prove $g r_{k}\left(K_{3}: s \cdot K_{4}+e,(k-s) \cdot K_{3}\right)>g(k, s)$ by construction. Let $G_{0}$ be a single vertex and $G_{1}$ be a monochromatic copy of $K_{4}$ using color 1 . If $s$ is even, then we will begin with $G_{0}$ and iteratively construct Gallai-colored graphs. If $s$ is odd, then we will begin with $G_{1}$ and iteratively construct Gallai-colored graphs. Suppose we have constructed $G_{i}$ for some $i<k$. Let $G^{\prime}$ be a 2-edge-colored $K_{5}$ using colors $i+1$ and $i+2$ which contains no monochromatic copy of $K_{3}$, and $G^{\prime \prime}$ be a 2-edge-colored $K_{17}$ using colors $i+1$ and $i+2$ which contains no monochromatic copy of $K_{4}$. We construct $G_{i+2}$ or $G_{i+1}$ based on the following rules:

(1) if $i \leq s-2$, then we construct $G_{i+2}$ such that $G_{i+2}=G^{\prime \prime}\left(17 \cdot G_{i}\right)$;

(2) if $s \leq i \leq k-2$, then we construct $G_{i+2}$ such that $G_{i+2}=G^{\prime}\left(5 \cdot G_{i}\right)$;

(3) if $i=k-1$, then we construct $G_{i+1}$ by joining two copies of $G_{i}$ with edges using color $k$.

Finally, we obtain a Gallai- $k$-colored graph $G_{k}$ of order $g(k, s)$, which contains neither a monochromatic copy of $K_{4}+e$ in any of the first $s$ colors nor a monochromatic copy of $K_{3}$ in any of the last $k-s$ colors.

\subsection{Proof of the upper bounds}

In this section, we shall prove $g r_{k}\left(K_{3}: s \cdot K_{4}+e,(k-s) \cdot K_{3}\right) \leq g(k, s)+1$ by induction on $k+s$. The case $k=1$ is trivial, the case $k=2$ follows from Theorem 3.3, and the case $s=0$ follows from Theorem 2.7. So we may assume that the result holds for all $k^{\prime}+s^{\prime}<k+s$ and we will prove it for $k+s$, where $k \geq 3$ and $1 \leq s \leq k$.

Let $G$ be a Gallai- $k$-coloring of $K_{n}$, where $n:=g(k, s)+1$. For a contradiction, suppose that $G$ contains neither a monochromatic copy of $K_{4}+e$ in any of the first $s$ colors nor a monochromatic copy of $K_{3}$ in any of the last $k-s$ colors. By Theorem 1.4, let $V_{1}, V_{2}, \ldots, V_{t}(t \geq 2)$ be a Gallai partition of $V(G)$. We choose such a partition so that $t$ is minimum. We may assume that red and blue are the two colors used between these parts, where red and blue are two of the $k$ colors. Note that $n=g(k, s)+1 \geq 21$ since $k \geq 3$ and $1 \leq s \leq k$. 
Claim 3.1. $t \geq 4$.

Proof. If $t=3$, then at least two of the colors $c\left(V_{1}, V_{2}\right), c\left(V_{1}, V_{3}\right)$ and $c\left(V_{2}, V_{3}\right)$ are the same color, say $c\left(V_{1}, V_{2}\right)=c\left(V_{1}, V_{3}\right)$. This implies that $V_{1}$ and $V(G) \backslash V_{1}$ form a Gallai partition with exactly two parts, contradicting the minimality of $t$. Thus $t=2$, and we may assume that $c\left(V_{1}, V_{2}\right)$ is red without loss of generality.

If there is no red edge within both $V_{1}$ and $V_{2}$, then $G\left[V_{1}\right]$ and $G\left[V_{2}\right]$ are two Gallai- $(k-1)$-colorings. By the induction hypothesis, if red is one of the first $s$ colors, then

$$
\begin{aligned}
n & =\left|V_{1}\right|+\left|V_{2}\right| \leq 2 \cdot g(k-1, s-1) \\
& = \begin{cases}2 \cdot 17^{(s-1) / 2} \cdot 5^{(k-s) / 2}, & \text { if } s-1 \text { is even and } k-s \text { is even, } \\
2 \cdot 2 \cdot 17^{(s-1) / 2} \cdot 5^{(k-s-1) / 2}, & \text { if } s-1 \text { is even and } k-s \text { is odd, } \\
2 \cdot 8 \cdot 17^{(s-2) / 2} \cdot 5^{(k-s-1) / 2}, & \text { if } s-1 \text { is odd and } k-s \text { is odd, } \\
2 \cdot 4 \cdot 17^{(s-2) / 2} \cdot 5^{(k-s) / 2}, & \text { if } s-1 \text { is odd and } k-s \text { is even }\end{cases} \\
& \leq g(k, s),
\end{aligned}
$$

a contradiction. If red is one of the last $k-s$ colors, then

$$
\begin{aligned}
n & =\left|V_{1}\right|+\left|V_{2}\right| \leq 2 \cdot g(k-1, s) \\
& = \begin{cases}2 \cdot 17^{s / 2} \cdot 5^{(k-s-1) / 2}, & \text { if } s \text { is even and } k-s-1 \text { is even, } \\
2 \cdot 2 \cdot 17^{s / 2} \cdot 5^{(k-s-2) / 2}, & \text { if } s \text { is even and } k-s-1 \text { is odd, } \\
2 \cdot 8 \cdot 17^{(s-1) / 2} \cdot 5^{(k-s-2) / 2}, & \text { if } s \text { is odd and } k-s-1 \text { is odd, } \\
2 \cdot 4 \cdot 17^{(s-1) / 2} \cdot 5^{(k-s-1) / 2}, & \text { if } s \text { is odd and } k-s-1 \text { is even }\end{cases} \\
& \leq g(k, s),
\end{aligned}
$$

a contradiction.

If $G\left[V_{1}\right]$ or $G\left[V_{2}\right]$ contains a red edge, then red is one of the first $s$ colors. Without loss of generality, we may assume that $G\left[V_{1}\right]$ contains a red edge. In order to avoid a red copy of $K_{4}+e$, there is no red edge within $V_{2}$ and there is no red copy of $K_{3}$ within $V_{1}$ (recall that $n \geq 21$ ). By the induction hypothesis, 
we have

$$
\begin{aligned}
n & =\left|V_{1}\right|+\left|V_{2}\right| \leq g(k, s-1)+g(k-1, s-1) \\
& =\left\{\begin{array}{r}
8 \cdot 17^{(s-2) / 2} \cdot 5^{(k-s) / 2}+4 \cdot 17^{(s-2) / 2} \cdot 5^{(k-s) / 2}, \\
\text { if } s \text { is even and } k-s \text { is even, } \\
4 \cdot 17^{(s-2) / 2} \cdot 5^{(k-s+1) / 2}+8 \cdot 17^{(s-2) / 2} \cdot 5^{(k-s-1) / 2}, \\
\text { if } s \text { is even and } k-s \text { is odd, } \\
17^{(s-1) / 2} \cdot 5^{(k-s+1) / 2}+2 \cdot 17^{(s-1) / 2} \cdot 5^{(k-s-1) / 2}, \\
\text { if } s \text { is odd and } k-s \text { is odd, } \\
2 \cdot 17^{(s-1) / 2} \cdot 5^{(k-s) / 2}+17^{(s-1) / 2} \cdot 5^{(k-s) / 2,}, \\
\text { if } s \text { is odd and } k-s \text { is even }
\end{array}\right. \\
& \leq g(k, s),
\end{aligned}
$$

a contradiction. This completes the proof of Claim 3.1.

We define $R$ to be a 2-edge-coloring of $K_{t}$ with $V(R)=\left\{v_{1}, v_{2}, \ldots, v_{t}\right\}$ and $c\left(v_{i} v_{j}\right)=c\left(V_{i}, V_{j}\right)$ for any $1 \leq i<j \leq t$ (i.e., $R$ is the reduced graph of the Gallai partition). Note that if $R$ contains a 2-edge-colored subgraph $H$, then $G$ also contains a copy of $H$ (in fact, $G$ contains a blow-up of $H$ ). For each $i \in[t]$, let $N_{i}^{r}:=\left\{j \in[t] \backslash\{i\}: c\left(v_{i} v_{j}\right)\right.$ is red $\}, N_{i}^{b}:=\left\{j \in[t] \backslash\{i\}: c\left(v_{i} v_{j}\right)\right.$ is blue $\}$, $d_{i}^{r}:=\left|N_{i}^{r}\right|$ and $d_{i}^{b}:=\left|N_{i}^{b}\right|$. By Claim 3.1 and the minimality of $t$, we have $d_{i}^{r} \geq 1$ and $d_{i}^{b} \geq 1$ for every $i \in[t]$. We claim that at least one of red and blue is among the first $s$ colors. Indeed, if both red and blue are among the last $k-s$ colors, then $R$ contains no monochromatic copy of $K_{3}$. So $t \leq r\left(K_{3}, K_{3}\right)-1=5$. Moreover, for every $i \in[t]$, since $d_{i}^{r} \geq 1$ and $d_{i}^{b} \geq 1$, there is no red edge and no blue edge within $V_{i}$ in $G$. By the induction hypothesis, we have $n=\sum_{i=1}^{t}\left|V_{i}\right| \leq 5 \cdot g(k-2, s) \leq g(k, s)$, a contradiction.

Let $\mathscr{R}:=\left\{i \in[t]: G\left[V_{i}\right]\right.$ contains a red edge $\}$ and $\mathscr{B}:=\left\{i \in[t]: G\left[V_{i}\right]\right.$ contains a blue edge $\}$. Let $x_{0}:=|[t] \backslash(\mathscr{R} \cup \mathscr{B})|, x_{1}:=|\mathscr{R} \triangle \mathscr{B}|$ and $x_{2}:=$ $|\mathscr{R} \cap \mathscr{B}|$, so $t=x_{0}+x_{1}+x_{2}$. We have the following simple facts.

Fact 3.1. The following six statements hold. 
(1) For any $i \in \mathscr{R}$ (resp., $i \in \mathscr{B}$ ), we have that $v_{i}$ is not contained in any red copy of $K_{3}$ (resp., blue copy of $K_{3}$ ) in $R$.

(2) For any $i, j \in \mathscr{R}$ (resp., $i, j \in \mathscr{B})$ with $i \neq j$, we have that $c\left(V_{i}, V_{j}\right)$ is blue (resp., red).

(3) For any $i \in \mathscr{R}$ (resp., $i \in \mathscr{B})$, we have $d_{i}^{r} \leq 3$ (resp., $d_{i}^{b} \leq 3$ ).

(4) For any $i \in[t]$, we have $d_{i}^{r} \leq 8$ and $d_{i}^{b} \leq 8$.

(5) For any $i \in[t], G\left[V_{i}\right]$ contains neither a red copy of $K_{3}$ nor a blue copy of $K_{3}$.

(6) $x_{2} \leq 1$.

Proof. By the symmetry of red and blue, we will only prove the red case for (1)-(5). Note that if red is one of the last $k-s$ colors, then Fact 3.1 holds clearly. So we may assume that red is one of the first $s$ colors.

(1) If there exists an $i \in \mathscr{R}$ such that $v_{i}$ is contained in a red copy of $K_{3}$ in $R$, say $v_{i} v_{j} v_{\ell} v_{i}$, then in order to avoid a red copy of $K_{4}+e$, we have that $c\left(V_{i} \cup V_{j} \cup V_{\ell}, V(G) \backslash\left(V_{i} \cup V_{j} \cup V_{\ell}\right)\right)$ is blue. By the minimality of $t$, we have $t=2$, contradicting Claim 3.1.

(2) If there exist some $i, j \in \mathscr{R}$ with $i \neq j$ such that $c\left(V_{i}, V_{j}\right)$ is red, then for avoiding a red copy of $K_{4}+e$, we have that $c\left(V_{i} \cup V_{j}, V(G) \backslash\left(V_{i} \cup V_{j}\right)\right)$ is blue. By the minimality of $t$, we have $t=2$, contradicting Claim 3.1.

(3) If there exists an $i \in \mathscr{R}$ such that $d_{i}^{r} \geq 4$, then $\left\{v_{j}: j \in N_{i}^{r}\right\}$ forms a blue copy of $K_{d_{i}^{r}}$ by (1). In order to avoid a blue copy of $K_{4}+e$, we have $d_{i}^{r}=4$ and $c\left(\bigcup_{j \in N_{i}^{r}} V_{j}, \bigcup_{\ell \in[t] \backslash N_{i}^{r}} V_{\ell}\right)$ is red. By the minimality of $t$, we have $t=2$, contradicting Claim 3.1.

(4) Suppose $d_{i}^{r} \geq 9$ for some $i \in[t]$. In order to avoid a red copy of $K_{4}+e$, there is no red copy of $K_{3}$ in $R\left[\left\{v_{j}: j \in N_{i}^{r}\right\}\right]$. Since $r\left(K_{3}, K_{4}+e\right)=9$, there is a blue copy of $K_{4}+e$ (and thus a blue copy of $K_{3}$ ), a contradiction.

(5) Suppose that $G\left[V_{i}\right]$ contains a red copy of $K_{3}$ for some $i \in[t]$. Since $d_{i}^{r} \geq 1$, we may assume that $c\left(V_{i}, V_{j}\right)$ is red for some $j \in[t] \backslash\{i\}$. In order to avoid a red copy of $K_{4}+e$, we have that $c\left(V_{i} \cup V_{j}, V(G) \backslash\left(V_{i} \cup V_{j}\right)\right)$ is blue. By the minimality of $t$, we have $t=2$, contradicting Claim 3.1. 
(6) If $x_{2}=|\mathscr{R} \cap \mathscr{B}| \geq 2$, then we can derive a contradiction by (2).

We divide the rest of the proof into two cases according to where red and blue are in the list of colors.

Case 1. Red is among the first $s$ colors and blue is among the last $k-s$ colors.

In this case, there is no red copy of $K_{4}+e$ and no blue copy of $K_{3}$ in $G$. Since $r\left(K_{4}+e, K_{3}\right)=9$, we have $4 \leq t \leq 8$. Recall that $d_{i}^{r} \geq 1$ and $d_{i}^{b} \geq 1$ for every $i \in[t]$. So there is no blue edge within each $V_{i}$. Thus $|\mathscr{B}|=0$, $x_{1}=|\mathscr{R}|, x_{2}=0$ and $x_{0}=t-x_{1}$. We claim that $x_{1} \leq 2$, since otherwise if $|\mathscr{R}| \geq 3$, then there is a blue copy of $K_{3}$ by Fact 3.1 (2).

For each $i \in \mathscr{R}, G\left[V_{i}\right]$ contains no red copy of $K_{3}$ by Fact 3.1 (5). By the induction hypothesis, we have

$$
\begin{aligned}
\left|V_{i}\right| & \leq g(k-1, s-1) \\
& = \begin{cases}17^{(s-1) / 2} \cdot 5^{(k-s) / 2}, & \text { if } s-1 \text { is even and } k-s \text { is even, } \\
2 \cdot 17^{(s-1) / 2} \cdot 5^{(k-s-1) / 2}, & \text { if } s-1 \text { is even and } k-s \text { is odd, } \\
8 \cdot 17^{(s-2) / 2} \cdot 5^{(k-s-1) / 2}, & \text { if } s-1 \text { is odd and } k-s \text { is odd, } \\
4 \cdot 17^{(s-2) / 2} \cdot 5^{(k-s) / 2}, & \text { if } s-1 \text { is odd and } k-s \text { is even }\end{cases} \\
& \leq \frac{1}{4} g(k, s) .
\end{aligned}
$$

For each $i \in[t] \backslash(\mathscr{R} \cup \mathscr{B})$, by the induction hypothesis, we have

$$
\begin{aligned}
\left|V_{i}\right| & \leq g(k-2, s-1) \\
& = \begin{cases}17^{(s-1) / 2} \cdot 5^{(k-s-1) / 2}, & \text { if } s-1 \text { is even and } k-s-1 \text { is even, } \\
2 \cdot 17^{(s-1) / 2} \cdot 5^{(k-s-2) / 2}, & \text { if } s-1 \text { is even and } k-s-1 \text { is odd, } \\
8 \cdot 17^{(s-2) / 2} \cdot 5^{(k-s-2) / 2}, & \text { if } s-1 \text { is odd and } k-s-1 \text { is odd, } \\
4 \cdot 17^{(s-2) / 2} \cdot 5^{(k-s-1) / 2}, & \text { if } s-1 \text { is odd and } k-s-1 \text { is even }\end{cases} \\
& \leq \frac{1}{8} g(k, s) .
\end{aligned}
$$

Thus $n \leq\left(x_{1} / 4+x_{0} / 8\right) g(k, s)$. It suffices to prove that $x_{1} / 4+x_{0} / 8 \leq 1$. If $x_{1} \leq 8-t$, then $x_{1} / 4+x_{0} / 8=\left(2 x_{1}+x_{0}\right) / 8=\left(x_{1}+t\right) / 8 \leq 1$. Thus 
we may assume $x_{1} \geq 9-t$. Recall that we have $t \leq 8$ and $x_{1} \leq 2$ in this case. So $|\mathscr{R}|=x_{1} \geq 1$ and $7 \leq t \leq 8$. For any $i \in \mathscr{R}$, we have $d_{i}^{r} \leq 2$ for avoiding a blue copy of $K_{3}$ and by Fact 3.1 (1). Thus $d_{i}^{b} \geq 4$. Since there is no blue copy of $K_{3}$, we have that $\left\{v_{j}: j \in N_{i}^{b}\right\}$ forms a red copy of $K_{d_{i}^{b}}$. Then $c\left(\bigcup_{j \in N_{i}^{b}} V_{j}, \bigcup_{\ell \in[t] \backslash N_{i}^{b}} V_{\ell}\right)$ is blue. By the minimality of $t$, we have $t=2$, contradicting Claim 3.1.

Case 2. Both red and blue are among the first $s$ colors.

In this case, we have $4 \leq t \leq 17$ since $r\left(K_{4}+e, K_{4}+e\right)=18$. Moreover, we have $s \geq 2$ and thus $g(k, s) \geq 34$ (recall that $k \geq 3$ ). By the induction hypothesis, for every $i \in[t] \backslash(\mathscr{R} \cup \mathscr{B})$, we have $\left|V_{i}\right| \leq g(k-2, s-2)=\frac{1}{17} g(k, s)$. For any $i \in[t], G\left[V_{i}\right]$ contains neither a red copy of $K_{3}$ nor a blue copy of $K_{3}$ by Fact 3.1 (5). Thus for each $i \in \mathscr{R} \cap \mathscr{B}$, by the induction hypothesis, we have $\left|V_{i}\right| \leq g(k, s-2)=\frac{5}{17} g(k, s)$. And for each $i \in \mathscr{R} \triangle \mathscr{B}$, we have

$$
\begin{aligned}
\left|V_{i}\right| & \leq g(k-1, s-2) \\
& = \begin{cases}17^{(s-2) / 2} \cdot 5^{(k-s+1) / 2}, & \text { if } s-2 \text { is even and } k-s+1 \text { is even, } \\
2 \cdot 17^{(s-2) / 2} \cdot 5^{(k-s) / 2}, & \text { if } s-2 \text { is even and } k-s+1 \text { is odd, } \\
8 \cdot 17^{(s-3) / 2} \cdot 5^{(k-s) / 2}, & \text { if } s-2 \text { is odd and } k-s+1 \text { is odd, } \\
4 \cdot 17^{(s-3) / 2} \cdot 5^{(k-s+1) / 2}, & \text { if } s-2 \text { is odd and } k-s+1 \text { is even }\end{cases} \\
& \leq \frac{5}{34} g(k, s) .
\end{aligned}
$$

Thus $n \leq\left(5 x_{2} / 17+5 x_{1} / 34+x_{0} / 17\right) g(k, s)$. It suffices to prove that $10 x_{2}+5 x_{1}+2 x_{0}=2 t+8 x_{2}+3 x_{1} \leq 34$.

Claim 3.2. $x_{2}=0$.

Proof. By Fact 3.1 (6), we have $x_{2} \leq 1$. For a contradiction, suppose $\mathscr{R} \cap \mathscr{B}=$ $\{1\}$. By Fact 3.1 (3), we have $d_{1}^{r} \leq 3$ and $d_{1}^{b} \leq 3$, so $t \leq 7$. If $t \leq 5$, then $2 t+8 x_{2}+3 x_{1} \leq 10+8+12 \leq 34$. If $6 \leq t \leq 7$, then we may assume that $d_{1}^{r}=3$ without loss of generality, say $N_{1}^{r}=\{2,3,4\}$. By Fact 3.1 (1), we have that $c\left(v_{2} v_{3}\right)=c\left(v_{3} v_{4}\right)=c\left(v_{2} v_{4}\right)$ is blue. By Fact 3.1 (1) and (2), we have $2,3,4 \notin \mathscr{R} \cup \mathscr{B}$. Thus $x_{1} \leq t-4$, so $2 t+8 x_{2}+3 x_{1} \leq 8+5 t-12 \leq 34$. 
Claim 3.3. $|\mathscr{R}| \leq 3$ and $|\mathscr{B}| \leq 3$. If $|\mathscr{R}|=3$ (resp., $|\mathscr{B}|=3$ ), then $|\mathscr{B}| \leq 1$ (resp., $|\mathscr{R}| \leq 1$ ).

Proof. If $|\mathscr{R}| \geq 4$ (resp., $|\mathscr{B}| \geq 4$ ), then $G$ contains a blue (resp., red) $K_{2,2,2,2}$ by Fact 3.1 (2). This implies a monochromatic copy of $K_{4}+e$ in $G$. Thus $|\mathscr{R}| \leq 3$ and $|\mathscr{B}| \leq 3$.

If $|\mathscr{R}|=3$ and $2 \leq|\mathscr{B}| \leq 3$, then $R\left[\left\{v_{i}: i \in \mathscr{R}\right\}\right]$ and $R\left[\left\{v_{i}: i \in \mathscr{B}\right\}\right]$ form a blue clique and a red clique (by Fact 3.1 (2)), respectively. By Fact 3.1 (1), for any $i \in \mathscr{R}$ (resp., $i \in \mathscr{B}$ ), there is at most one red (resp., blue) edge between $v_{i}$ and $\left\{v_{j}: j \in \mathscr{B}\right\}$ (resp., $\left\{v_{j}: j \in \mathscr{R}\right\}$ ). Thus there are at most $|\mathscr{R}|+|\mathscr{B}|<|\mathscr{R}||\mathscr{B}|$ edges between $\left\{v_{i}: i \in \mathscr{R}\right\}$ and $\left\{v_{i}: i \in \mathscr{B}\right\}$, a contradiction. Therefore, if $|\mathscr{R}|=3$, then $|\mathscr{B}| \leq 1$, and similarly, if $|\mathscr{B}|=3$, then $|\mathscr{R}| \leq 1$.

By Claims 3.2 and 3.3, we have $x_{2}=0$ and $x_{1}=|\mathscr{R}|+|\mathscr{B}| \leq 4$. If $t \leq 11$, then $2 t+8 x_{2}+3 x_{1} \leq 22+0+12=34$. If $13 \leq t \leq 17$, then $|\mathscr{R}|=|\mathscr{B}|=0$ by Fact 3.1 (3) and (4), so $2 t+8 x_{2}+3 x_{1} \leq 34+0+0=34$. Thus $t=12$. We have $x_{1}=|\mathscr{R}|+|\mathscr{B}|=4$; otherwise $2 t+8 x_{2}+3 x_{1} \leq 24+0+9 \leq 34$. Then we further have $|\mathscr{R}| \geq 1$ and $|\mathscr{B}| \geq 1$ by Claim 3.3. Without loss of generality, let $1 \in \mathscr{R}, 2 \in \mathscr{B}$ and let $c\left(V_{1}, V_{2}\right)$ be blue. Moreover, by Fact 3.1 (3) and (4), we have $d_{1}^{r}=3, d_{1}^{b}=8, d_{2}^{b}=3$ and $d_{2}^{r}=8$. We may further assume that $c\left(V_{1}, V_{3} \cup V_{4} \cup \cdots \cup V_{9}\right)$ is blue. By Fact 3.1 (1), we have $c\left(V_{2}, V_{3} \cup V_{4} \cup \cdots \cup V_{9}\right)$ is red. Since $r\left(K_{3}, K_{3}\right)=6$, there is either a red copy of $K_{3}$ or a blue copy of $K_{3}$ in $R\left[\left\{v_{3}, v_{4}, \ldots, v_{9}\right\}\right]$. Then there is either a red copy of $K_{4}+e$ or a blue copy of $K_{4}+e$ in $G$. This contradiction completes the proof of Theorem 3.2.

\subsection{A short survey on known Gallai-Ramsey numbers}

In this section, we present a summary, to the best of our knowledge, of all the known exact values of Gallai-Ramsey numbers for rainbow triangles. In addition, we give an analysis of the known results and provide some open problems.

Known exact values. It was shown by Gyárfás et al. [91] that $g r_{k}\left(K_{3}: H\right)$ is linear in $k$ for bipartite graphs $H$ (except if $H$ is a star), and $g r_{k}\left(K_{3}: H\right)$ is 
exponential in $k$ for non-bipartite graphs $H$. This is similar to the multicolor Ramsey numbers for graphs. It is well-known that $r_{k}(H)$ is polynomial in $k$ for bipartite graphs $H$ and at least exponential in $k$ for non-bipartite graphs $H$. We summarize all the known exact values of Gallai-Ramsey numbers $g r_{k}\left(K_{3}: H\right)$ in Tables 3.1 and 3.2.

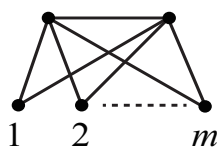

$B_{m}$

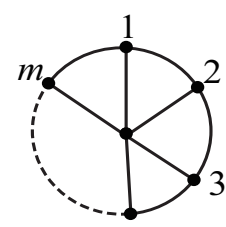

$W_{m}$

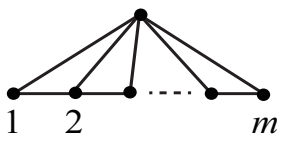

$\hat{K}_{m}$

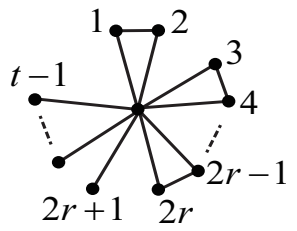

$S_{t}^{r}$

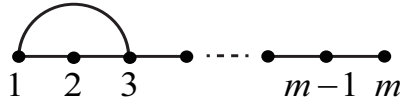

$P_{m}^{*}$

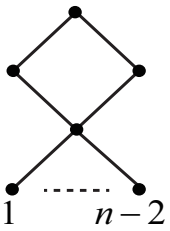

$F_{2, n}$

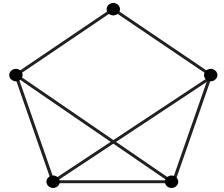

$H_{0}$
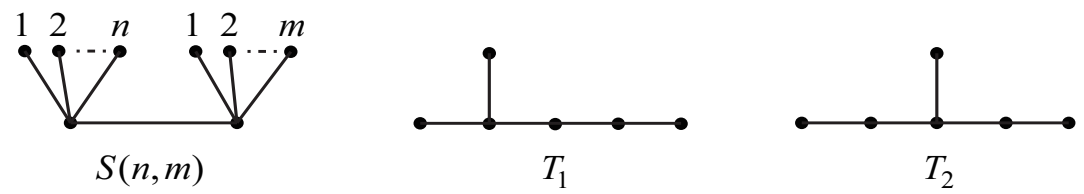

Figure 3.1: An illustration of some special graphs.

For the sake of notation, we introduce some additional families of graphs (see Figure 3.1). For $m \geq 1$, the book graph $B_{m}$ is defined by $B_{m}=K_{2} \vee \overline{K_{m}}$. For $m \geq 2$, the kipas $\hat{K}_{m}$ is defined by $\hat{K}_{m}=K_{1} \vee P_{m}$. For $m \geq 3$, let $P_{m}^{*}$ denote the graph consisting of the path $P_{m}$ with an extra edge joining one end and the vertex at distance 2 along the path from that end. Note that the graph $P_{5}^{*}$ was denoted by $F_{5}$ in Figure 2.1. For $m \geq 3$, the wheel $W_{m}$ is defined by $W_{m}=K_{1} \vee C_{m}$. For $r \geq 1$ and $t \geq 2 r+1$, the graph $S_{t}^{r}$ is obtained from a star of order $t$ by adding an extra $r$ independent edges between the leaves of the star. Note that the graphs $S_{5}^{1}$ and $S_{5}^{2}$ were denoted by $F_{4}$ and $F_{8}$ in Figure 2.1, respectively. For $n \geq m \geq 0$, the double star $S(n, m)$ is the graph consisting of the disjoint union of stars $K_{1, n}$ and $K_{1, m}$ with an extra edge joining their centers. Note that the graph $S(2,1)$ was denoted by $F_{2}$ in Figure 2.1. The 
graphs $F_{9}, F_{10}, F_{12}, F_{13}$ and $F_{2, n}$ were defined in Chapter 2. The graph $H_{0}$ is a 5 -vertex graph obtained by removing the edges of a copy of $K_{2} \cup P_{3}$ from $K_{5}$. The graph $T_{1}$ is a 6 -vertex tree obtained from a path of length 4 by attaching a pendant edge at the second vertex along the path. The graph $T_{2}$ is a 6 -vertex tree obtained from a path of length 4 by attaching a pendant edge at the third vertex along the path.

\begin{tabular}{|l|l|l|}
\hline$H$ & $g r_{k}\left(K_{3}: H\right)(k \geq 3)$ & Cite \\
\hline$K_{1, t}(t \geq 3)$ & $\begin{array}{l}(5 t-3) / 2, \quad \text { if } t \text { is odd, } \\
5 t / 2-3, \quad \text { if } t \text { is even }\end{array}$ & {$[91]$} \\
\hline$P_{n}$ & $\left.\frac{n-2}{2}\right] k+\left[\frac{n}{2}\right]+1$ & {$[178]$} \\
\hline$C_{4}(n+4$ & {$[72]$} \\
\hline$C_{2 n}(n \geq 3)$ & $(n-1) k+n+1$ & {$[178]$} \\
\hline $\begin{array}{l}K_{2, n}(2 \leq n \leq 17 \text { or } \\
n \text { is large enough) }\end{array}$ & $k-2+r_{2}\left(K_{2, n}\right)$ & {$[122,175]$} \\
\hline$K_{3,3}$ & $2 k+14$ & {$[175]$} \\
\hline $\begin{array}{l}K_{3, n}(4 \leq n \leq 5 \text { or } \\
n \text { is large enough) }\end{array}$ & $2(k-2)+r_{2}\left(K_{3, n}\right)$ & {$[122]$} \\
\hline$n K_{2}$ & $(n-1) k+n+1$ & {$[72,175]$} \\
\hline $\begin{array}{l}S(n, m)(m \geq 1 \text { and } \\
\text { even } n \geq 6 m+7)\end{array}$ & $\frac{5 n}{2}+m(k-3)+1$ & {$[106]$} \\
\hline $\begin{array}{l}S(n, m)(m \geq 1 \text { and } \\
\text { odd } n \geq 6 m+7)\end{array}$ & $\frac{5(n-1)}{2}+m(k-3)+2$ & {$[106]$} \\
\hline$F_{2,3}$ & $k+4$ & Theorem 2.3 \\
\hline$F_{2,4}$ & $k+5$ & Theorem 2.3 \\
\hline$F_{2,5}$ & $k+9$ & Theorem 2.3 \\
\hline$S(2,1)$ & $k+4$ & {$[72]$} \\
\hline$T_{1}, S(3,1)$ & $k+5$ & {$[72]$} \\
\hline$T_{2}, S(2,2)$ & $2 k+4$ & {$[72]$} \\
\hline & & \\
\hline
\end{tabular}

Table 3.1: Gallai-Ramsey numbers for bipartite graphs.

Two driving conjectures. Given a connected bipartite graph $H$, let $s(H)$ be the order of the smallest part and let $b(H)$ be the order of the largest part of the bipartition of $H$. For general connected bipartite graphs, the following conjecture was posed in [175]. 


\begin{tabular}{|c|c|c|}
\hline$H$ & $g r_{k}\left(K_{3}: H\right)(k \geq 3)$ & Cite \\
\hline$K_{3}$ & $\begin{cases}5^{k / 2}+1, & \text { if } k \text { is even, } \\
2 \cdot 5^{(k-1) / 2}+1, & \text { if } k \text { is odd }\end{cases}$ & {$[36,91]$} \\
\hline$K_{4}$ & $\begin{cases}17^{k / 2}+1, & \text { if } k \text { is even, } \\
3 \cdot 17^{(k-1) / 2}+1, & \text { if } k \text { is odd }\end{cases}$ & {$[123]$} \\
\hline$K_{4}+e$ & $\begin{cases}17^{k / 2}+1, & \text { if } k \text { is even, } \\
4 \cdot 17^{(k-1) / 2}+1, & \text { if } k \text { is odd }\end{cases}$ & Theorem 3.1 \\
\hline$C_{2 n+1}(n \geq 2)$ & $n \cdot 2^{k}+1$ & {$[172,178]$} \\
\hline$F_{8}$ & $\begin{cases}\frac{83}{2} \cdot 5^{(k-4) / 2}+\frac{1}{2}, & \text { if } k \text { is even, } \\
4 \cdot 5^{(k-1) / 2}+1, & \text { if } k \text { is odd }\end{cases}$ & {$[138]$} \\
\hline$F_{9}, F_{10}$ & $\begin{cases}8 \cdot 5^{(k-2) / 2}+1, & \text { if } k \text { is even } \\
4 \cdot 5^{(k-1) / 2}+1, & \text { if } k \text { is odd }\end{cases}$ & Theorem 2.1 \\
\hline$F_{12}, F_{13}$ & $\begin{array}{ll}9 \cdot 5^{(k-2) / 2}+1, & \text { if } k \text { is even, } \\
4 \cdot 5^{(k-1) / 2}+1, & \text { if } k \text { is odd }\end{array}$ & Theorem 2.4 \\
\hline $\begin{array}{l}B_{m} \\
(2 \leq m \leq 5)\end{array}$ & $\begin{array}{ll}\left(r_{2}\left(B_{m}\right)-1\right) \cdot 5^{\frac{k-2}{2}}+1, & \text { if } k \text { is even, } \\
2 \cdot\left(r_{2}\left(B_{m}\right)-1\right) \cdot 5^{\frac{k-3}{2}}+1, & \text { if } k \text { is odd }\end{array}$ & {$[181]$} \\
\hline$W_{4}$ & $\begin{array}{ll}14 \cdot 5^{(k-2) / 2}+1, & \text { if } k \text { is even, } \\
28 \cdot 5^{(k-3) / 2}+1, & \text { if } k \text { is odd }\end{array}$ & {$[163]$} \\
\hline$\hat{K}_{4}$ & $\begin{array}{ll}2 \cdot 5^{k / 2}, & \text { if } k \text { is even, } \\
4 \cdot 5^{(k-1) / 2}+1, & \text { if } k \text { is odd }\end{array}$ & {$[180]$} \\
\hline$P_{m}^{*}(m \geq 4)$ & $\begin{array}{ll}2(m-1) \cdot 5^{(k-2) / 2}+1, & \text { if } k \text { is even, } \\
(m-1) \cdot 5^{(k-1) / 2}+1, & \text { if } k \text { is odd }\end{array}$ & {$[173]$} \\
\hline$S_{t}^{1}(t \geq 4)$ & $\begin{array}{ll}2(t-1) \cdot 5^{(k-2) / 2}+1, & \text { if } k \text { is even, } \\
(t-1) \cdot 5^{(k-1) / 2}+1, & \text { if } k \text { is odd }\end{array}$ & {$[173]$} \\
\hline$S_{6}^{2}$ & $\begin{array}{ll}2 \cdot 5^{k / 2}+\frac{1}{4} \cdot 5^{(k-2) / 2}+\frac{3}{4}, & \text { if } k \text { is even, } \\
\left\lceil\frac{51}{10} \cdot 5^{(k-1) / 2}+\frac{1}{2}\right\rceil, & \text { if } k \text { is odd }\end{array}$ & {$[139]$} \\
\hline$S_{8}^{2}$ & $\begin{array}{ll}14 \cdot 5^{\frac{k-2}{2}}+\frac{1}{2} \cdot 5^{\frac{k-4}{2}}+\frac{1}{2}, & \text { if } k \text { is even, } \\
7 \cdot 5^{\frac{k-1}{2}}+\frac{1}{4} \cdot 5^{\frac{k-3}{2}}+\frac{3}{4}, & \text { if } k \text { is odd }\end{array}$ & {$[139]$} \\
\hline$H_{0}$ & $\begin{array}{l}9 \cdot 5^{(k-2) / 2}+1, \quad \text { if } k \text { is even, } \\
4 \cdot 5^{(k-1) / 2}+1, \quad \text { if } k \text { is odd }\end{array}$ & {$[180]$} \\
\hline
\end{tabular}

Table 3.2: Gallai-Ramsey numbers for non-bipartite graphs. 
Conjecture 3.1 (Wu et al. [175]). For any integer $k \geq 2$ and connected bipartite graph $H$, if $r_{2}(H) \geq 3 b(H)-2$, then

$$
g r_{k}\left(K_{3}: H\right)=(s(H)-1)(k-2)+r_{2}(H) .
$$

The assumption $r_{2}(H) \geq 3 b(H)-2$ in Conjecture 3.1 is necessary (consider the cases $H=K_{1, t}$ with $t \geq 5, H=S(n, m)$ with $m \geq 1$ and $n \geq 6 m+7$, and $H=F_{2, n}$ with even $n \geq 8$ or odd $n \geq 5$ ). This conjecture is true for all connected bipartite graphs $H$ shown in Table 3.1 with $r_{2}(H) \geq 3 b(H)-2$. In particular, this conjecture holds for paths of even order, even cycles, $K_{2, n}$ ( $2 \leq n \leq 17$ or $n$ large enough), $K_{3, n}$ ( $3 \leq n \leq 5$ or $n$ large enough), $T_{2}$ and $S(2,2)$. Furthermore, there exist graphs $H$ which violate $r_{2}(H) \geq 3 b(H)-2$ but satisfy $g r_{k}\left(K_{3}: H\right)=(s(H)-1)(k-2)+r_{2}(H)$. For example, paths of odd order, $F_{2,3}, F_{2,4}, S(2,1), T_{1}$ and $S(3,1)$. The Ramsey numbers of all the graphs shown in Table 3.1 can be found in the survey [152] and the references therein.

A driving conjecture for non-bipartite graphs is Conjecture 1.1 which concerns complete graphs. This conjecture was only confirmed for the complete graphs $K_{3}$ and $K_{4}$. The conjectured exact values (which are also lower bounds) for $g r_{k}\left(K_{3}: K_{t}\right)$ are expressions in terms of the 2-colored Ramsey numbers. If one can prove an upper bound on $g r_{k}\left(K_{3}: K_{t}\right)$, then it will yield an upper bound on $r_{2}\left(K_{t}\right)$. Moreover, Conjecture 1.1 has a close relationship with the multicolor Erdős-Hajnal conjecture [75].

The influence of the parity of the number of colors. As shown in Tables 3.1 and 3.2, an interesting observation is that for all known exact values of $g r_{k}\left(K_{3}: H\right)$, if $H$ is bipartite, then $g r_{k}\left(K_{3}: H\right)$ does not depend on the parity of $k$; if $H$ is non-bipartite but not a cycle, then $g r_{k}\left(K_{3}: H\right)$ depends on the parity of $k$. We do not know why this phenomenon happens. One possible reason might be the extremal structures, that is, the Gallai- $k$-colorings of $K_{n}$ without monochromatic copies of $H$, where $n=g r_{k}\left(K_{3}: H\right)-1$. Although we do not know whether the extremal structure for each graph $H$ is unique or not, we can give an analysis based on the extremal structures that were given in the references shown in Tables 3.1 and 3.2.

For most bipartite graphs $H$, the extremal structures were constructed as 
follows (see also [175]). Let $G_{2}$ be a 2-edge-colored complete graph of order $r_{2}(H)-1$ without monochromatic copies of $H$. We then construct $G_{3}, \ldots, G_{k}$, where for each $3 \leq i \leq k, G_{i}$ is obtained by adding $s(H)-1$ new vertices to $G_{i-1}$ such that all the new edges get color $i$. The resulting $k$-edge-colored complete graph $G_{k}$ has order $(s(H)-1)(k-2)+r_{2}(H)-1$ and contains no monochromatic copies of $H$. Note that such constructions do not depend on the parity of $k$.

Next, we consider non-bipartite graphs. Before providing the constructions, we first introduce some additional notation and results. Given two graphs $H$ and $F$, we say that $F$ is a homomorphic copy of $H$ if there is a map $\varphi: V(H) \rightarrow V(F)$ such that $\varphi(u) \varphi(v) \in E(F)$ for each edge $u v \in E(H)$. Let $m(H)$ be the smallest integer $n$ such that every 2-edge-coloring of $K_{n}$ contains a monochromatic homomorphic copy of $H$. We claim that if $K_{3} \subseteq H$ and $K_{3}$ is a homomorphic copy of $H$, then $m(H)=6$. Indeed, since $K_{3}$ is a homomorphic copy of $H$, then $m(H) \leq r_{2}\left(K_{3}\right)=6$. Since $K_{3} \subseteq H$, we have that $C_{5}$ is not a homomorphic copy of $H$. The 2-edge-coloring of $K_{5}$ in which each color induces a $C_{5}$ implies that $m(H)>5$. Thus $m(H)=6$. Note that for $H \in\left\{K_{3}, F_{8}, F_{9}, F_{10}, F_{12}, F_{13}, B_{m}, W_{4}, \hat{K}_{m}, P_{m}^{*}, S_{t}^{r}, H_{0}\right\}$, we have $m(H)=6$. For any non-bipartite graph $H$, we claim that if both $K_{3}$ and $C_{5}$ are homomorphic copies of $H$, then $m(H)=5$. Indeed, since every 2-edge-coloring of $K_{5}$ contains a monochromatic copy of $F$ for some $F \in\left\{K_{3}, C_{5}\right\}$, we have $m(H) \leq 5$. Since $H$ is non-bipartite, we have that $P_{4}$ is not a homomorphic copy of $H$. The 2-edge-coloring of $K_{4}$ in which each color induces a $P_{4}$ implies that $m(H)>4$. Thus $m(H)=5$. Note that for $n \geq 2$, we have $m\left(C_{2 n+1}\right)=5$.

For most of the non-bipartite graphs $H$, the extremal structures depend on the parity of $k$. We first consider the case that $k$ is even (the constructions can also be found in [136]). Let $G_{2}$ be a 2-edge-colored complete graph of order $r_{2}(H)-1$ without monochromatic copies of $H$. We construct $G_{4}, \ldots, G_{k}$ as follows. For each $4 \leq 2 i \leq k$, let $G^{\prime}$ be a 2-edge-colored complete graph of order $m(H)-1$ using colors $2 i-1$ and $2 i$ which contains no monochromatic homomorphic copies of $H$. We construct $G_{2 i}$ such that $G_{2 i}$ is the blow-up $G^{\prime}\left((m(H)-1) \cdot G_{2 i-2}\right)$ of $G^{\prime}$. Finally, we obtain a $k$-edge-coloring $G_{k}$ of $K_{n}$ containing neither a rainbow $K_{3}$ nor a monochromatic $H$, where $n=$ $\left(r_{2}(H)-1\right) \cdot(m(H)-1)^{(k-2) / 2}$. Note that for any graph $H$ in Table 3.2 except 
for $F_{8}, \hat{K}_{4}, S_{6}^{2}$ and $S_{8}^{2}$, this construction is sharp for even $k \geq 4$.

We next consider the case that $k$ is odd. There are two constructions in this case. We now introduce the first construction (see also [136]). Similar to the case that $k$ is even, we obtain a Gallai- $(k-1)$-coloring $G_{k-1}$ of $K_{n_{k-1}}$ without monochromatic $H$, where $n_{k-1}=\left(r_{2}(H)-1\right) \cdot(m(H)-1)^{(k-3) / 2}$. Let $G^{\prime \prime}$ be a monochromatic complete graph of order $\chi(H)-1$ with color $k$, where $\chi(H)$ is the chromatic number of $H$. We construct $G_{k}$ such that $G_{k}$ is the blow-up $G^{\prime \prime}\left((\chi(H)-1) \cdot G_{k-1}\right)$ of $G^{\prime \prime}$. Then $G_{k}$ is a $k$-edge-coloring of $K_{n}$ containing neither a rainbow $K_{3}$ nor a monochromatic $H$, where $n=(\chi(H)-$ $1) \cdot\left(r_{2}(H)-1\right) \cdot(m(H)-1)^{(k-3) / 2}$. We next introduce the second construction. Let $G_{1}$ be a monochromatic complete graph of order $|V(H)|-1$ with color 1. We construct $G_{3}, \ldots, G_{k}$ as follows. For each $3 \leq 2 i+1 \leq k$, let $G^{\prime \prime \prime}$ be a 2-edge-colored complete graph of order $m(H)-1$ using colors $2 i$ and $2 i+1$ which contains no monochromatic homomorphic copies of $H$. We construct $G_{2 i+1}$ such that $G_{2 i+1}$ is the blow-up $G^{\prime \prime \prime}\left((m(H)-1) \cdot G_{2 i-1}\right)$ of $G^{\prime \prime \prime}$. Finally, we obtain a $k$-edge-coloring $G_{k}$ of $K_{n}$ with $n=(|V(H)|-1) \cdot(m(H)-1)^{(k-1) / 2}$ containing neither a rainbow $K_{3}$ nor a monochromatic $H$. Note that for $H \in\left\{K_{3}, K_{4}, C_{2 n+1}\right\}$, both of the two constructions above are sharp for odd $k \geq 3$. For $H \in\left\{B_{m}(2 \leq m \leq 5), W_{4}\right\}$, the first construction is sharp for odd $k \geq 3$. For $H \in\left\{K_{4}+e, F_{8}, F_{9}, F_{10}, F_{12}, F_{13}, \hat{K}_{4}, P_{m}^{*}, S_{t}^{1}, H_{0}\right\}$, the second construction is sharp for odd $k \geq 3$.

The influence of the constraint on rainbow triangles. In view of the additional constraint on rainbow triangles, we have $g r_{k}\left(K_{3}: H\right) \leq r_{k}(H)$ for every $H$ and $k$. For most of the graphs shown in Tables 3.1 and 3.2, the inequality is strict whenever $k \geq 3$. However, there exist graphs $H$ such that equality holds for $k \geq 3$ (the constraint on rainbow triangles has no influence). This phenomenon happens for both bipartite and non-bipartite graphs. An example for bipartite graphs is the matching $n K_{2}$. For any $n \geq 1$ and $k \geq 1$, we have $g r_{k}\left(K_{3}: n K_{2}\right)=r_{k}\left(n K_{2}\right)=(n-1) k+n+1[42,72,175]$. An example for non-bipartite graphs is the odd cycle $C_{2 n+1}$. For any fixed $k$ and sufficiently large $n$, we have $g r_{k}\left(K_{3}: C_{2 n+1}\right)=r_{k}\left(C_{2 n+1}\right)=n \cdot 2^{k}+1[102,172,178]$. Let $\mathscr{A}$ be the set of all pairs $(H, k)$ such that $g r_{k}\left(K_{3}: H\right)=r_{k}(H)$. A natural problem is to determine the set $\mathscr{A}$. An equivalent problem is for what graphs $H$ and integers $k \geq 3$, there exists an extremal structure for $r_{k}(H)$ 
which is rainbow triangle-free. When we study the Gallai-Ramsey numbers $g r_{k}\left(K_{3}: H\right)$, we in fact only investigate the Gallai- $k$-colorings. However, it was shown in [17] that for $n$ large enough and every $k$ with $3 \leq k \leq n-1$, the number of Gallai- $k$-colorings of $K_{n}$ is much smaller than the number of $k$-edge-colorings of $K_{n}$. This property may force $g r_{k}\left(K_{3}: H\right)<r_{k}(H)$ to hold for certain graphs $H$ of sufficiently large order and integers $k \geq 3$. 


\section{Chapter 4}

\section{Two extremal problems and results related to Gallai-colorings}

In this chapter, we consider two natural extremal problems related to Gallaicolorings. In the first part we determine upper and lower bounds for the maximum number of edges that are not contained in any rainbow triangle or monochromatic triangle in a $k$-edge-coloring of $K_{n}$. In the second part, for $n \geq g r_{k}\left(K_{3}: K_{3}\right)$, we determine upper and lower bounds for the minimum number of monochromatic triangles in a Gallai-k-coloring of $K_{n}$.

\subsection{Introduction}

Recall that the Gallai-Ramsey number $g r_{k}\left(K_{3}: H\right)$ is the minimum integer $n$ such that every $k$-edge-coloring of $K_{n}$ contains either a rainbow copy of $K_{3}$ or a monochromatic copy of $H$. As we have seen in the previous two chapters, it is far from trivial to determine exact values of Gallai-Ramsey numbers, even for a small graph $H$. There the lower bounds were obtained by showing specific $k$-edge-colorings of complete graphs that do not admit a rainbow copy of $K_{3}$ or a monochromatic copy of $H$. Instead of disallowing a rainbow copy of $K_{3}$ 
and a monochromatic copy of $H$, it is natural to consider the related problem of determining the maximum number of edges that are not contained in any rainbow copy of $K_{3}$ or monochromatic copy of $H$. The analogous problem for classical Ramsey numbers was considered in $[107,128,133]$; in these papers the authors studied the maximum number of edges not contained in any monochromatic copy of $H$ over all $k$-edge-colorings of $K_{n}$. For $k \geq 2$, let $f_{k}(n, H)$ denote the maximum number of edges not contained in any rainbow triangle or monochromatic copy of $H$, over all $k$-edge-colorings of $K_{n}$. The first part of this chapter is devoted to obtaining upper and lower bounds on $f_{k}\left(n, K_{3}\right)$.

Let $e x(n, H)$ be the maximum number of edges of an $H$-free graph of order $n$, i.e., the Turán number of $H$. By Turán's Theorem, the unique $K_{r+1}$-free graph on $n$ vertices with ex $\left(n, K_{r+1}\right)$ edges is the Turán graph $T_{r}(n)$, i.e., the complete $r$-partite graph on $n$ vertices with class sizes as equal as possible. Let $t(n, r)$ be the number of edges of $T_{r}(n)$. Note that we have the trivial upper bound $f_{k}(n, H) \leq t\left(n, g r_{k}\left(K_{3}: H\right)-1\right)$. For the case $H=K_{3}$, we will prove the following theorem.

Theorem 4.1. For any real number $\delta>0$, there exists an $n_{0}$ such that for all integers $n \geq n_{0}$, we have $t\left(n, g r_{k-1}\left(K_{3}: K_{3}\right)-1\right) \leq f_{k}\left(n, K_{3}\right)<t\left(n, g r_{k-1}\left(K_{3}\right.\right.$ : $\left.\left.K_{3}\right)-1\right)+\delta n^{2}$.

We conjecture that the lower bound on $f_{k}\left(n, K_{3}\right)$ in Theorem 4.1 is in fact the exact value of $f_{k}\left(n, K_{3}\right)$. Moreover, we can generalize this result to a general graph $H$ (see Theorem 4.10).

The second part of this chapter is devoted to the Gallai-Ramsey multiplicity problem. By the definition of the Gallai-Ramsey number, if $n \geq g r_{k}\left(K_{3}: H\right)$, then any Gallai-k-coloring of $K_{n}$ contains a monochromatic copy of $H$. In fact, there could be more than one monochromatic copy of $H$. In light of this, it is natural to consider the minimum number of monochromatic copies of $H$ (as an unlabeled graph) in a Gallai-k-coloring of $K_{n}$. Let $g_{k}(H, n)$ denote the minimum number of monochromatic copies of $H$ taken over all Gallai- $k$-colorings of $K_{n}$. The analogous problem for classical Ramsey numbers is known as the Ramsey multiplicity problem, that is, to consider the minimum number $M_{k}(H, n)$ of monochromatic copies of $H$ taken over all 
$k$-edge-colorings of $K_{n}$ (see $[45,52,74,96]$ for some recent results). With the additional restriction imposed on Gallai-colorings, it is obvious that $g_{k}(H, n) \geq$ $M_{k}(H, n)$. In 1959, Goodman [86] proved the following classical result concerning $M_{2}\left(K_{3}, n\right)$.

Theorem 4.2 (Goodman [86]). For any positive integer $n$, we have

$$
M_{2}\left(K_{3}, n\right)= \begin{cases}n(n-2)(n-4) / 24, & \text { if } n \text { is even, } \\ n(n-1)(n-5) / 24, & \text { if } n \equiv 1 \bmod 4, \\ (n+1)(n-3)(n-4) / 24, & \text { if } n \equiv 3 \bmod 4 .\end{cases}
$$

For the case of 3-edge-colorings, Cummings et al. [52] proved the following result, using flag algebras and a probabilistic argument.

Theorem 4.3 (Cummings et al. [52]). There exists an integer $n_{0}$ such that for $n \geq n_{0}$, if we write $n=5 m+r$ for nonnegative integers $m$ and $r$ with $0 \leq r \leq 4$, then

$$
M_{3}\left(K_{3}, n\right)=r\left(\begin{array}{c}
m+1 \\
3
\end{array}\right)+(5-r)\left(\begin{array}{c}
m \\
3
\end{array}\right) .
$$

Our next result shows that $g_{3}\left(K_{3}, n\right)=M_{3}\left(K_{3}, n\right)$ for $n$ sufficiently large, and gives upper and lower bounds for $g_{k}\left(K_{3}, n\right)$ for other values of $k$.

Theorem 4.4. For any integer $n \geq g r_{k}\left(K_{3}: K_{3}\right)$, we write $n=5^{\lfloor(k-1) / 2\rfloor} m+r$, where $m$ and $r$ are nonnegative integers with $0 \leq r \leq 5^{\lfloor(k-1) / 2\rfloor}-1$. Then

$$
g_{k}\left(K_{3}, n\right) \leq \begin{cases}r\left(\begin{array}{c}
m+1 \\
3
\end{array}\right)+\left(5^{(k-1) / 2}-r\right)\left(\begin{array}{c}
m \\
3
\end{array}\right), & \text { if } k \text { is odd }, \\
r M_{2}\left(K_{3}, m+1\right)+\left(5^{(k-2) / 2}-r\right) M_{2}\left(K_{3}, m\right), & \text { if } k \text { is even. }\end{cases}
$$

Moreover, let $s_{0}=1$ if $k$ is odd, and $s_{0}=2$ if $k$ is even. Then

$$
g_{k}\left(K_{3}, n\right) \geq \frac{s_{0} n(n-1)(n-2)}{g r_{k}\left(K_{3}: K_{3}\right)\left(g r_{k}\left(K_{3}: K_{3}\right)-1\right)\left(g r_{k}\left(K_{3}: K_{3}\right)-2\right)} .
$$

In general, we conjecture that the above upper bound on $g_{k}\left(K_{3}, n\right)$ in Theorem 4.4 is in fact the exact value of $g_{k}\left(K_{3}, n\right)$, but we can only verify this for the following cases: (1) $k=3$ and $n$ sufficiently large, (2) $k \geq 3$ and $n=$ $g r_{k}\left(K_{3}: K_{3}\right)$, (3) $k$ is odd and $g r_{k}\left(K_{3}: K_{3}\right) \leq n \leq g r_{k}\left(K_{3}: K_{3}\right)+5^{(k-1) / 2}-1$. 


\subsection{Preliminaries}

In the following, we will introduce the Regularity Lemma, Embedding Lemma and Slicing Lemma that will be used in our proof of Theorem 4.1. Given a graph $F$ and two disjoint nonempty sets $X, Y \subseteq V(F)$, the density of $(X, Y)$ is defined to be

$$
d(X, Y):=\frac{|E(X, Y)|}{|X||Y|} .
$$

We say that $(X, Y)$ is $\varepsilon$-regular if for any $X^{\prime} \subseteq X$ and $Y^{\prime} \subseteq Y$ with $\left|X^{\prime}\right| \geq \varepsilon|X|$ and $\left|Y^{\prime}\right| \geq \varepsilon|Y|$, we have $\left|d\left(X^{\prime}, Y^{\prime}\right)-d(X, Y)\right| \leq \varepsilon$. For a positive real number $d$, we say that an $\varepsilon$-regular pair $(X, Y)$ is $(\varepsilon, d)$-regular if $d(X, Y) \geq d$.

Lemma 4.5 (Multicolor Regularity Lemma, see e.g. [108, 128, 168]). For any real $\varepsilon>0$ and positive integers $k$ and $m$, there exist $n^{\prime}$ and $M$, such that every $k$-edge-colored graph $F$ with $n \geq n^{\prime}$ vertices admits a partition $V_{1}, V_{2}, \ldots, V_{t}$ of $V(F)$ satisfying

(1) $m \leq t \leq M$;

(2) for all $1 \leq i<j \leq t$, we have ||$V_{i}|-| V_{j}|| \leq 1$; and

(3) for all but at most $\varepsilon\left(\begin{array}{l}t \\ 2\end{array}\right)$ pairs $(i, j)$, the pair $\left(V_{i}, V_{j}\right)$ is $\varepsilon$-regular for each color.

We call the partition as given in Lemma 4.5 a multicolored $\varepsilon$-regular partition. Given $\varepsilon, d>0$, a $k$-edge-colored graph $F$ and a partition $V_{1}, V_{2}, \ldots, V_{t}$ of $V(F)$, we define the reduced graph $R=R(d)$ as follows: $V(R)=\{1,2, \ldots, t\}$ and $i$ and $j$ are adjacent in $R$ if $\left(V_{i}, V_{j}\right)$ is $\varepsilon$-regular for each color and there exists a color with density at least $d$ in $E\left(V_{i}, V_{j}\right)$. Moreover, we define the multicolored reduced graph $R^{c}=R^{c}(d)$ as follows: $V\left(R^{c}\right)=V(R), E\left(R^{c}\right)=E(R)$, and for each edge $i j \in E\left(R^{c}\right), i j$ is assigned an arbitrary color $c_{0}$ such that $\left(V_{i}, V_{j}\right)$ has density at least $d$ with respect to the subgraph of $F$ induced by the edges of color $c_{0}$. Note that the reduced graph for an $\varepsilon$-regular partition defined above is different from the reduced graph for a Gallai partition defined in Section 1.3.1.

Given two graphs $G$ and $H$, we say that $G$ is a homomorphic copy of $H$ if there is a map $\varphi: V(H) \rightarrow V(G)$ such that $\varphi(u) \varphi(v) \in E(G)$ for each 
edge $u v \in E(H)$. Note that $K_{s}$ is a homomorphic copy of $H$ if and only if $s \geq \chi(H)$, where $\chi(H)$ is the chromatic number of $H$. We will use the following consequence of the Embedding Lemma. Lemma 4.6 below is in fact a corollary of Lemma 2.4 in [100].

Lemma 4.6 (Multicolor Embedding Lemma, see e.g. [100, 101, 108]). For every $d>0$, any positive integer $k$ and any graph $G$, there exist $\varepsilon=\varepsilon(k, d, G)>$ 0 and a positive integer $n_{0}=n_{0}(k, d, G)$ with the following property. Suppose that $F$ is a $k$-edge-colored graph on $n \geq n_{0}$ vertices with a multicolored $\varepsilon$-regular partition $V_{1}, V_{2}, \ldots, V_{t}$ which defines the multicolored reduced graph $R^{c}=R^{c}(d)$. If $R^{c}$ contains a monochromatic homomorphic copy of $G$, then $F$ contains a monochromatic copy of $G$. If $R^{c}$ contains a rainbow copy of $G$, then $F$ contains a rainbow copy of $G$.

Lemma 4.7 (Slicing Lemma, see e.g. $[108,128]$ ). Let $0<\varepsilon, \alpha, d<1$ with $\varepsilon \leq \min \{d, \alpha, 1 / 2\}$. If a pair $(X, Y)$ is $(\varepsilon, d)$-regular, then for any $X^{\prime} \subseteq X$ and $Y^{\prime} \subseteq Y$ with $\left|X^{\prime}\right| \geq \alpha|X|$ and $\left|Y^{\prime}\right| \geq \alpha|Y|$, we have that $\left(X^{\prime}, Y^{\prime}\right)$ is an $\left(\varepsilon^{\prime}, d-\varepsilon\right)$-regular pair, where $\varepsilon^{\prime}:=\max \{2 \varepsilon, \varepsilon / \alpha\}$.

Finally, we consider the Turán number. It is well-known that ex $\left(n, K_{r+1}\right)=$ $t(n, r)=(1-1 / r)\left(\begin{array}{l}n \\ 2\end{array}\right)+o\left(n^{2}\right)$. In fact, if $n \equiv p(\bmod r)$ where $0 \leq p \leq r-1$, then $t(n, r)=(1-1 / r) n^{2} / 2+(p-r) p /(2 r)$. Thus $(1-1 / r) n^{2} / 2-r / 8 \leq$ $t(n, r) \leq(1-1 / r) n^{2} / 2$. We will use this more precise bound in our proofs.

\subsection{Results related to the number of edges}

For the proof of Theorem 4.1, we first define the following variant of the Gallai-Ramsey number. Given a set $V$ and an integer $k \leq|V|$, let $\left(\begin{array}{c}V \\ \leq k\end{array}\right)$ (resp., $\left(\begin{array}{l}V \\ k\end{array}\right)$ ) be the set of all nonempty subsets of $V$ of size at most $k$ (resp., size $k$ ).

Definition 4.1. For a graph $H$ and an integer $k \geq 2$, let $G R_{k}^{*}(H)$ be the minimum integer $n^{*}$ such that for every coloring $c:\left(\begin{array}{c}{\left[n^{*}\right]} \\ \leq 2\end{array}\right) \rightarrow[k]$, at least one of the following statements holds:

$\left(1^{*}\right)$ the restriction of $c$ to $\left(\begin{array}{c}{\left[n^{*}\right]} \\ 2\end{array}\right)$ contains either a rainbow triangle or a monochromatic homomorphic copy of $H$; 
$\left(2^{*}\right)$ for some $1 \leq i<j \leq n^{*}$, we have $c(\{i, j\})=c(\{i\})$ or $c(\{i, j\})=c(\{j\})$.

In other words, $G R_{k}^{*}(H)-1$ is the maximum integer $n^{* *}$ such that for the complete graph $K_{n^{* *}}$ with vertex set $\left[n^{* *}\right]$, there exists a coloring $c:\left(\begin{array}{c}{\left[n^{* *}\right]} \\ \leq 2\end{array}\right) \rightarrow$ $[k]$ satisfying

$\left(1^{* *}\right)$ the restriction of $c$ to $\left(\begin{array}{c}{\left[n^{* *}\right]} \\ 2\end{array}\right)$ is a Gallai-k-coloring without a monochromatic homomorphic copy of $H$; and

$\left(2^{* *}\right)$ for any $1 \leq i<j \leq n^{* *}$, we have $c(\{i, j\}) \neq c(\{i\})$ and $c(\{i, j\}) \neq c(\{j\})$.

For a set $\mathscr{H}$ of graphs, let $G R_{k}(\mathscr{H})$ denote the minimum integer $n$ such that every Gallai- $k$-coloring of $K_{n}$ contains a monochromatic copy of $H$ for some $H \in \mathscr{H}$.

Lemma 4.8. For a graph $H$, let $\mathscr{H}$ be the set of all homomorphic copies of $H$. Then

(1) $G R_{k}^{*}(H) \geq G R_{k-1}(\mathscr{H})$,

(2) $f_{k}(n, H) \geq t\left(n, G R_{k-1}(\mathscr{H})-1\right)$,

(3) if there exists a coloring c satisfying conditions $\left(1^{* *}\right)$ and $\left(2^{* *}\right)$ such that all elements of $\left(\begin{array}{c}{\left[G R_{k}^{*}(H)-1\right]} \\ 1\end{array}\right)$ use a single color, then $f_{k}(n, H) \geq t(n$, $\left.G R_{k}^{*}(H)-1\right)$.

Proof. Let $n_{k}^{*}:=G R_{k-1}(\mathscr{H})$. We first prove (1). Let $F$ be a Gallai- $(k-1)$ coloring of $K_{n_{k}^{*}-1}$ without a monochromatic copy of $H^{\prime}$ for any $H^{\prime} \in \mathscr{H}$. We color the vertices of $F$ with the $k$ th color and then we obtain a $k$-coloring of $\left(\begin{array}{c}{\left[n_{k}^{*}-1\right]} \\ \leq 2\end{array}\right)$ satisfying conditions $\left(1^{* *}\right)$ and $\left(2^{* *}\right)$. Thus $G R_{k}^{*}(H) \geq n_{k}^{*}$.

Next, we prove (2). Let $G$ be a Gallai- $(k-1)$-coloring of $K_{n_{k}^{*}-1}$ without a monochromatic copy of $H^{\prime}$ for any $H^{\prime} \in \mathscr{H}$. Let $V(G)=\left\{1,2, \ldots, n_{k}^{*}-1\right\}$ and let $G^{\prime}$ be the Turán graph $T_{n_{k}^{*}-1}(n)$ with parts $V_{1}, V_{2}, \ldots, V_{n_{k}^{*}-1}$. We color the edges of $G^{\prime}$ such that for any $1 \leq i<j \leq n_{k}^{*}-1$, we have $c_{G^{\prime}}\left(V_{i}, V_{j}\right)=c_{G}(i j)$. Let $G^{\prime \prime}$ be a $k$-edge-coloring of $K_{n}$ obtained by coloring the edges within each part using color $k$ from the above $(k-1)$-edge-coloring of $G^{\prime}$. We claim that all the edges between the $n_{k}^{*}-1$ parts are neither contained in a rainbow copy of $K_{3}$ nor in a monochromatic copy of $H$ in $G^{\prime \prime}$. Indeed, note that there is no 
rainbow copy of $K_{3}$ using color $k$. Thus if $G^{\prime \prime}$ contains a rainbow copy of $K_{3}$, then $G$ is not a Gallai-coloring, a contradiction. If there is an edge $e$ between these $n_{k}^{*}-1$ parts such that $e$ is contained in a monochromatic copy of $H$, then $G$ contains a monochromatic homomorphic copy of $H$, a contradiction. Thus $f_{k}(n, H) \geq\left|E\left(G^{\prime}\right)\right|=t\left(n, n_{k}^{*}-1\right)$.

Finally, we prove (3). Let $n_{k}:=G R_{k}^{*}(H)-1$. Let $c$ be a coloring as in the statement of the lemma, and we may assume that all elements of $\left(\begin{array}{c}{\left[n_{k}\right]} \\ 1\end{array}\right)$ are colored by color 1 . Note that the restriction of $c$ to $\left(\begin{array}{c}{\left[n_{k}\right]} \\ 2\end{array}\right)$ is a Gallai- $(k-1)$ coloring without a monochromatic homomorphic copy of $H$. Let $W$ be the Turán graph $T_{n_{k}}(n)$ with parts $V_{1}, V_{2}, \ldots, V_{n_{k}}$. We color the edges of $W$ such that $c_{W}\left(V_{i}, V_{j}\right)=c(\{i, j\})$ for any $1 \leq i<j \leq n_{k}$. Let $W^{\prime}$ be a $k$-edge-coloring of $K_{n}$ obtained by coloring the edges within each part using color 1 from the above $(k-1)$-edge-coloring of $W$. It is easy to check that all the edges between the $n_{k}$ parts are neither contained in a rainbow copy of $K_{3}$ nor in a monochromatic copy of $H$ in $W^{\prime}$. Thus $f_{k}(n, H) \geq|E(W)|=t\left(n, n_{k}\right)$.

Note that we have $G R_{k}^{*}(H)=G R_{k-1}(\mathscr{H})=2$ whenever $H$ is a bipartite graph, where $\mathscr{H}$ is the set of all homomorphic copies of $H$. A natural question is for which non-bipartite graph $H$ it holds that $G R_{k}^{*}(H)=G R_{k-1}(\mathscr{H})$ ? We can verify that $K_{3}$ is such a graph.

Lemma 4.9. Let $\mathscr{H}\left(K_{3}\right)$ be the set of all homomorphic copies of $K_{3}$. For any integer $k \geq 2$, we have $G R_{k}^{*}\left(K_{3}\right)=G R_{k-1}\left(\mathscr{H}\left(K_{3}\right)\right)=g r_{k-1}\left(K_{3}: K_{3}\right)$.

Proof. For every graph $H^{\prime} \in \mathscr{H}\left(K_{3}\right)$, we have that $H^{\prime}$ contains $K_{3}$ as a subgraph by the definition. Thus $G R_{k-1}\left(\mathscr{H}\left(K_{3}\right)\right) \geq g r_{k-1}\left(K_{3}: K_{3}\right)$. Moreover, by Lemma 4.8 (1), we have $G R_{k}^{*}\left(K_{3}\right) \geq G R_{k-1}\left(\mathscr{H}\left(K_{3}\right)\right) \geq g r_{k-1}\left(K_{3}: K_{3}\right)$.

For $k \geq 2$, let $n_{k}^{*}:=g r_{k-1}\left(K_{3}: K_{3}\right)$, and we will prove $G R_{k}^{*}\left(K_{3}\right) \leq n_{k}^{*}$ by induction on $k$. When $k=2$, we have $G R_{2}^{*}\left(K_{3}\right)=3=n_{2}^{*}$ clearly. Suppose that for all $2 \leq k^{\prime} \leq k-1$, we have $G R_{k^{\prime}}^{*}\left(K_{3}\right) \leq n_{k^{\prime}}^{*}$. We will prove it for $k^{\prime}=k$. Let $n$ be the maximum integer such that there is a coloring $c:\left(\begin{array}{c}{[n]} \\ \leq 2\end{array}\right) \rightarrow[k]$ satisfying conditions $\left(1^{* *}\right)$ and $\left(2^{* *}\right)$. It suffices to show that $n \leq n_{k}^{*}-1$. By Theorem 1.4, there is a Gallai partition $V_{1}, V_{2}, \ldots, V_{m}(m \geq 2)$ of $[n]$. Note that $K_{3} \in \mathscr{H}\left(K_{3}\right)$. For avoiding a monochromatic copy of $K_{3}$, we have $m \leq 5$. We choose such a partition so that $m$ is minimum. Let $R$ be an edge-coloring 
of a complete graph with $V(R)=\left\{v_{1}, v_{2}, \ldots, v_{m}\right\}$ and $c\left(v_{i} v_{j}\right)=c\left(V_{i}, V_{j}\right)$ for any $i \neq j$.

If $m=5$ (resp., $m=4$ ), then $R$ is the unique 2-edge-coloring of $K_{5}$ without a monochromatic copy of $K_{3}$ (resp., $R$ is one of the two 2-edge-colorings of $K_{4}$ without a monochromatic copy of $K_{3}$ ). Then there is no edge using color 1 or 2 within each part $V_{i}$ for avoiding a monochromatic copy of $K_{3}$, and there is no vertex using color 1 or 2 within each part $V_{i}$ by condition $\left(2^{* *}\right)$. Thus if $k=3$, then $n \leq 5=n_{3}^{*}-1$, and if $k \geq 4$, then $n \leq 5\left(G R_{k-2}^{*}\left(K_{3}\right)-1\right) \leq$ $5\left(g r_{k-3}\left(K_{3}: K_{3}\right)-1\right) \leq n_{k}^{*}-1$ by the induction hypothesis and Theorem 2.7. If $m=3$, then at least two of the colors $c\left(V_{1}, V_{2}\right), c\left(V_{1}, V_{3}\right)$ and $c\left(V_{2}, V_{3}\right)$ are the same color, say $c\left(V_{1}, V_{2}\right)=c\left(V_{1}, V_{3}\right)$. This implies that $V_{1}$ and $V_{2} \cup V_{3}$ form a Gallai partition with exactly two parts, contradicting the minimality of $m$. If $m=2$, then we may assume $c\left(V_{1}, V_{2}\right)=1$. Then color 1 cannot be used on $\left(\begin{array}{c}V_{1} \\ \leq 2\end{array}\right)$ and $\left(\begin{array}{c}V_{2} \\ \leq 2\end{array}\right)$. Thus $n \leq 2\left(G R_{k-1}^{*}\left(K_{3}\right)-1\right) \leq 2\left(g r_{k-2}\left(K_{3}: K_{3}\right)-1\right) \leq n_{k}^{*}-1$ by the induction hypothesis and Theorem 2.7 .

By Lemma 4.9, we have $G R_{k}^{*}\left(K_{3}\right)=G R_{k-1}\left(\mathscr{H}\left(K_{3}\right)\right)$. As in the proof of Lemma 4.8 (1), we can construct an extremal coloring $\left(\begin{array}{c}{\left[G R_{k}^{*}\left(K_{3}\right)-1\right]} \\ \leq 2\end{array}\right) \rightarrow[k]$ satisfying conditions $\left(1^{* *}\right)$ and $\left(2^{* *}\right)$ in which we assign a single color to all elements of $\left(\begin{array}{c}{\left[G R_{k}^{*}\left(K_{3}\right)-1\right]} \\ 1\end{array}\right)$. It is worth noticing that not all the extremal colorings assign a single color to all singletons. For example, Figure 4.1 gives an extremal coloring of $G R_{4}^{*}\left(K_{3}\right)$ with two colors on singletons.

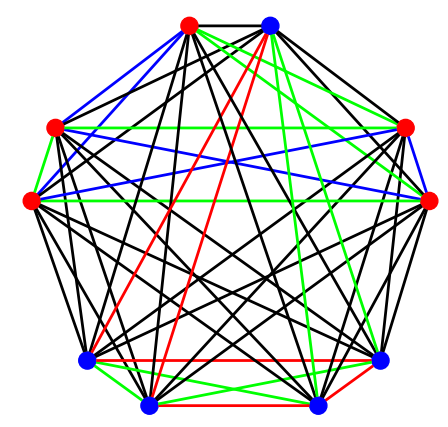

Figure 4.1: An extremal coloring of $G R_{4}^{*}\left(K_{3}\right)$ with two colors on singletons.

Now we have all the ingredients for our proof of Theorem 4.1. 
Proof of Theorem 4.1. The lower bound follows from Lemmas 4.8 (2) and 4.9. In the following, we will prove $f_{k}\left(n, K_{3}\right)<t\left(n, g r_{k-1}\left(K_{3}: K_{3}\right)-1\right)+\delta n^{2}$. Let $\operatorname{nim}_{k}\left(n, K_{3}\right)$ be the maximum number of edges not contained in any monochromatic copy of $K_{3}$ over all $k$-edge-colorings of $K_{n}$. Note that $f_{k}\left(n, K_{3}\right)$ $\leq \operatorname{nim}_{k}\left(n, K_{3}\right)$. For sufficiently large $n$, since $\operatorname{nim}_{2}\left(n, K_{3}\right)=t(n, 2)$ (proven in [107]) and $\operatorname{nim}_{3}\left(n, K_{3}\right)=t(n, 5)$ (proven in [128]), we have $f_{k}\left(n, K_{3}\right)=$ $t\left(n, g r_{k-1}\left(K_{3}: K_{3}\right)-1\right)$ for $k \in\{2,3\}$. In the following, we may assume $k \geq 4$.

Let $N_{k}:=g r_{k-1}\left(K_{3}: K_{3}\right)$. We choose $d$ such that $d \leq \delta / k$. Moreover, let $\varepsilon_{1}=\varepsilon_{1}\left(k, d / 2, K_{3}\right)$ and $n_{1}=n_{1}\left(k, d / 2, K_{3}\right)$ (resp., $\varepsilon_{2}=\varepsilon_{2}\left(k, d, K_{3}\right)$ and $\left.n_{2}=n_{2}\left(k, d, K_{3}\right)\right)$ be the values obtained by applying Lemma 4.6. Let $n_{1}^{\prime}$ and $M_{1}$ be the values obtained by applying Lemma 4.5 with $\varepsilon_{1}$ and $1 / \varepsilon_{1}$. Then we choose $\varepsilon$ such that $\varepsilon \leq \min \left\{\delta / 4, \varepsilon_{1} / M_{1}, \varepsilon_{2}, d / 2\right\}$. Let $n^{\prime}$ and $M$ be the values obtained by applying Lemma 4.5 with $\varepsilon$ and $1 / \varepsilon$. Furthermore, let $n_{0}=\max \left\{n^{\prime}, n_{1}^{\prime} M, \sqrt{\left(N_{k}-1\right) /(2 \delta)}, M M_{1} n_{1} / 3, n_{2}\right\}$ and $n \geq n_{0}$.

Let $F$ be a $k$-edge-coloring of $K_{n}$, and $F^{\prime}$ be the spanning subgraph of $F$ with $E\left(F^{\prime}\right)=\{e \in E(F): e$ is not contained in any rainbow or monochromatic copy of $\left.K_{3}\right\}$. For a contradiction, suppose $\left|E\left(F^{\prime}\right)\right| \geq t\left(n, N_{k}-1\right)+\delta n^{2}$. Let $V_{1}, V_{2}, \ldots, V_{t}$ be a partition of $V\left(F^{\prime}\right)$ obtained by applying Lemma 4.5 to $F^{\prime}$ with $\varepsilon$ and $1 / \varepsilon$, where $1 / \varepsilon \leq t \leq M$. Let $R=R(d)$ be the reduced graph. Since there are at most $\left(\begin{array}{c}n / t \\ 2\end{array}\right)$ edges within a part, at most $(n / t)^{2}$ edges between any two parts, and less than $k d(n / t)^{2}$ edges between a pair of parts with density less than $d$ for each color, we have

$$
\begin{aligned}
|E(R)| & >\frac{t\left(n, N_{k}-1\right)+\delta n^{2}-t\left(\begin{array}{c}
\frac{n}{t} \\
2
\end{array}\right)-\varepsilon\left(\begin{array}{c}
t \\
2
\end{array}\right)\left(\frac{n}{t}\right)^{2}-k d\left(\frac{n}{t}\right)^{2}\left(\begin{array}{c}
t \\
2
\end{array}\right)}{\left(\frac{n}{t}\right)^{2}} \\
& >\frac{t^{2}\left(\left(1-\frac{1}{N_{k}-1}\right) \frac{n^{2}}{2}-\frac{N_{k}-1}{8}+\delta n^{2}-\left(\frac{1}{t}+\varepsilon+k d\right) \frac{n^{2}}{2}\right)}{n^{2}} \\
& =\left(1-\frac{1}{N_{k}-1}+2 \delta-\frac{N_{k}-1}{4 n^{2}}-\frac{1}{t}-\varepsilon-k d\right) \frac{t^{2}}{2} \\
& \geq\left(1-\frac{1}{N_{k}-1}\right) \frac{t^{2}}{2},
\end{aligned}
$$

where the last inequality is by the choices of $n, d$ and $\varepsilon$. Therefore, we have 
$|E(R)| \geq t\left(t, N_{k}-1\right)+1$. So $R$ contains a copy $R^{\prime}$ of $K_{N_{k}}$. Without loss of generality, let $V\left(R^{\prime}\right)=\left\{1,2, \ldots, N_{k}\right\}$. Then for any $1 \leq i<j \leq N_{k}$, we have that $\left(V_{i}, V_{j}\right)$ is $\varepsilon$-regular for each color, and there exists a color $c_{i j}$ with density at least $d$ in $E\left(V_{i}, V_{j}\right)$.

For each $i \in\left[N_{k}\right]$, we have $\left|V_{i}\right|=n / t \geq\left(n_{1}^{\prime} M\right) / M=n_{1}^{\prime}$. Thus we can apply Lemma 4.5 with $\varepsilon_{1}$ and $1 / \varepsilon_{1}$ to $F\left[V_{i}\right]$ (note that here we consider $F\left[V_{i}\right]$, not only $\left.F^{\prime}\left[V_{i}\right]\right)$. Then there exist two subsets $V_{i, 1}, V_{i, 2} \subseteq V_{i}$ with $\left|V_{i, 1}\right|=\left|V_{i, 2}\right| \geq\left|V_{i}\right| / M_{1}$ such that $\left(V_{i, 1}, V_{i, 2}\right)$ is an $\left(\varepsilon_{1}, 1 / k\right)$-regular pair for some color $c_{i} \in[k]$. From the choice of $d$, we have $1 / k \geq d / 2$, so $\left(V_{i, 1}, V_{i, 2}\right)$ is an $\left(\varepsilon_{1}, d / 2\right)$-regular pair for color $c_{i}$. We define a coloring $\varphi:\left(\begin{array}{c}V\left(R^{\prime}\right) \\ \leq 2\end{array}\right) \rightarrow[k]$ such that $\varphi(\{i\})=c_{i}$ and $\varphi(\{i, j\})=c_{i j}$. Note that there might be more than one choice for $\varphi(\{i\})$ and $\varphi(\{i, j\})$, and we may choose an arbitrary one from these choices. By Lemma 4.9, we have $\left|V\left(R^{\prime}\right)\right|=N_{k}=g r_{k-1}\left(K_{3}: K_{3}\right)=$ $G R_{k}^{*}\left(K_{3}\right)$. Thus at least one of the following statements holds:

(1) $R^{\prime}$ contains a rainbow copy of $K_{3}$;

(2) $R^{\prime}$ contains a monochromatic homomorphic copy of $K_{3}$;

(3) $\varphi(\{i, j\})=\varphi(\{i\})$ for some $1 \leq i \neq j \leq N_{k}$.

If (1) or (2) holds, then there is a rainbow or monochromatic copy of $K_{3}$ in $F^{\prime}$ by Lemma 4.6, a contradiction. If (3) holds, then by applying Lemma 4.7 with $\alpha=1 / M_{1}$, we have that $\left(V_{j}, V_{i, 1}\right)$ and $\left(V_{j}, V_{i, 2}\right)$ are two $\left(\varepsilon M_{1}, d-\varepsilon\right)$-regular (and thus $\left(\varepsilon_{1}, d / 2\right)$-regular) pairs for color $c_{i}$. Thus $\left(V_{i, 1}, V_{i, 2}\right),\left(V_{j}, V_{i, 1}\right)$ and $\left(V_{j}, V_{i, 2}\right)$ are three $\left(\varepsilon_{1}, d / 2\right)$-regular pairs for color $c_{i}$. By Lemma 4.6, there is a monochromatic copy of $K_{3}$ which contains two edges of $F^{\prime}$, a contradiction.

By similar arguments as in the proof of Theorem 4.1, we can prove the following result for a general graph $H$. The main difference is that we need to find a copy of $K_{g}$ with $g=r_{k}\left(K_{\chi(H)-1}\right)$ in the reduced graph $R_{i}$ of $F\left(V_{i}\right)$. This is guaranteed by choosing appropriate parameters so that $\left|E\left(R_{i}\right)\right|>\operatorname{ex}\left(\left|V\left(R_{i}\right)\right|, K_{g}\right)$. We omit the details. 
Theorem 4.10. For any $\delta>0$, there exists an $n_{0}$ such that for all integers $n \geq$ $n_{0}$ and any graph $H$, we have $t\left(n, G R_{k-1}(\mathscr{H})-1\right) \leq f_{k}(n, H)<t\left(n, G R_{k}^{*}(H)-\right.$ $1)+\delta n^{2}$, where $\mathscr{H}$ is the set of all homomorphic copies of $H$.

\subsection{The Gallai-Ramsey multiplicity problem}

We first prove the upper bound in Theorem 4.4, by construction. Let $G_{2}$ be a 2edge-colored $K_{5}$ using colors 1 and 2 which contains no monochromatic copy of $K_{3}$, i.e., colors 1 and 2 induce two monochromatic copies of $C_{5}$. Suppose that $2 i<k-2$ and we have constructed a Gallai-(2i)-coloring $G_{2 i}$ of $K_{n_{2 i}}$ without a monochromatic copy of $K_{3}$, where $n_{2 i}:=5^{i}$. Let $G^{\prime}$ be a 2-edgecolored $K_{5}$ using colors $2 i+1$ and $2 i+2$ which contains no monochromatic copy of $K_{3}$. Let $G_{2 i+2}=G^{\prime}\left(5 \cdot G_{2 i}\right)$, i.e., $G_{2 i+2}$ is a blow-up of $G^{\prime}$. This way, when $k$ is odd (resp., $k$ is even), we obtain a Gallai- $(k-1)$-coloring $G_{k-1}$ of $K_{n_{k-1}}$ (resp., Gallai- $(k-2)$-coloring $G_{k-2}$ of $K_{n_{k-2}}$ ) without a monochromatic copy of $K_{3}$, where $n_{k-1}=5^{(k-1) / 2}$ (resp., $n_{k-2}=5^{(k-2) / 2}$ ). In the following, we will construct a Gallai- $k$-coloring $G_{k}$ from $G_{k-1}$ or $G_{k-2}$.

If $k$ is odd, then let $A$ (resp., $B$ ) be a monochromatic copy of $K_{m}$ (resp., $\left.K_{m+1}\right)$ using color $k$. Let $G_{k}=G_{k-1}\left(r \cdot B,\left(5^{(k-1) / 2}-r\right) \cdot A\right)$. Then $G_{k}$ is a Gallai- $k$-coloring of $K_{n}$ with $r\left(\begin{array}{c}m+1 \\ 3\end{array}\right)+\left(5^{(k-1) / 2}-r\right)\left(\begin{array}{c}m \\ 3\end{array}\right)$ monochromatic copies of $K_{3}$ (here we define $\left(\begin{array}{l}1 \\ 3\end{array}\right)=\left(\begin{array}{l}2 \\ 3\end{array}\right)=0$ for the sake of notation). If $k$ is even, then let $C$ be a 2-edge-coloring (using colors $k-1$ and $k$ ) of $K_{m}$ with $M_{2}\left(K_{3}, m\right)$ monochromatic copies of $K_{3}$, and let $D$ be a 2-edge-coloring (using colors $k-1$ and $k$ ) of $K_{m+1}$ with $M_{2}\left(K_{3}, m+1\right)$ monochromatic copies of $K_{3}$. Let $G_{k}=G_{k-2}\left(r \cdot D,\left(5^{(k-2) / 2}-r\right) \cdot C\right)$. Then $G_{k}$ is a Gallai-k-coloring of $K_{n}$ with $r M_{2}\left(K_{3}, m+1\right)+\left(5^{(k-2) / 2}-r\right) M_{2}\left(K_{3}, m\right)$ monochromatic copies of $K_{3}$. This completes the proof for the upper bound in Theorem 4.4.

It is worth noting that no matter whether $k$ is odd or even, the above extremal coloring is a blow-up of a complete graph of order $5^{\lfloor(k-1) / 2\rfloor}$ with a special edge-coloring. Recall that we have $g_{3}\left(K_{3}, n\right)=r\left(\begin{array}{c}m+1 \\ 3\end{array}\right)+(5-r)\left(\begin{array}{c}m \\ 3\end{array}\right)$ for sufficiently large $n$. An interesting fact is that the above sharpness example for $k=3$ is the unique Gallai-3-coloring of $K_{n}$ achieving the minimum number of monochromatic copies of $K_{3}$, which can be derived from a result of [52]. But 
when $k$ is an even number, the extremal colorings achieving the upper bound are not unique. For example, let $F$ be a 2-edge-coloring (using colors $k-1$ and $k$ ) of $K_{m+2}$ with $M_{2}\left(K_{3}, m+2\right)$ monochromatic copies of $K_{3}$. Since $M_{2}\left(K_{3}, m\right)+$ $M_{2}\left(K_{3}, m+2\right)=2 M_{2}\left(K_{3}, m+1\right)$ for any odd number $m$ by Theorem 4.2, we can also construct $G_{k}$ such that $G_{k}=G_{k-2}\left(1 \cdot F,(r-2) \cdot D,\left(5^{(k-2) / 2}-r+1\right) \cdot C\right)$. However, it is still a blow-up of a complete graph of order $5^{\lfloor(k-1) / 2\rfloor}$ with a special edge-coloring.

Before presenting our proof for the lower bound in Theorem 4.4, we first provide the exact value of $g_{k}\left(K_{3}, g r_{k}\left(K_{3}: K_{3}\right)\right)$.

Theorem 4.11. If $k$ is odd, then $g_{k}\left(K_{3}, g r_{k}\left(K_{3}: K_{3}\right)\right)=1$, and if $k$ is even, then $g_{k}\left(K_{3}, g r_{k}\left(K_{3}: K_{3}\right)\right)=2$.

Proof. By the definition of the Gallai-Ramsey number $g r_{k}\left(K_{3}: K_{3}\right)$, we have $g_{k}\left(K_{3}, g r_{k}\left(K_{3}: K_{3}\right)\right) \geq 1$. Moreover, it follows from the above extremal coloring that $g_{k}\left(K_{3}, g r_{k}\left(K_{3}: K_{3}\right)\right) \leq 1$ if $k$ is odd, and $g_{k}\left(K_{3}, g r_{k}\left(K_{3}: K_{3}\right)\right) \leq 2$ if $k$ is even. Thus it suffices to prove that $g_{k}\left(K_{3}, g r_{k}\left(K_{3}: K_{3}\right)\right) \geq 2$ when $k$ is even. We will prove this by induction on $k$. For $k=2$, the statement is trivial since $M_{2}\left(K_{3}, 6\right)=2$. We may assume that the statement holds for all even $k^{\prime} \leq k-2$ and we will prove it for $k(k \geq 4)$.

Let $F$ be a Gallai- $k$-coloring of $K_{g r_{k}\left(K_{3}: K_{3}\right)}$ and suppose (for a contradiction) that $F$ contains only one monochromatic copy of $K_{3}$. Using Theorem 1.4, let $V_{1}, V_{2}, \ldots, V_{t}(t \geq 2)$ be a Gallai partition of $V(F)$. We choose such a partition so that $t$ is minimum. We may assume that colors 1 and 2 are the two colors used between these parts. Let $R$ be a 2-edge-coloring of $K_{t}$ with $V(R)=\left\{v_{1}, v_{2}, \ldots, v_{t}\right\}$ and $c\left(v_{i} v_{j}\right)=c\left(V_{i}, V_{j}\right)$ for any $1 \leq i<j \leq t$. Since $M_{2}\left(K_{3}, 6\right)=2$, we have $t \leq 5$; otherwise $F$ contains at least two monochromatic copies of $K_{3}$.

If $2 \leq t \leq 3$, then we may assume that $t=2$ by the minimality of $t$ (since every edge-colored graph admitting a Gallai partition with three parts also admits a Gallai partition with two parts). Without loss of generality, let $c\left(V_{1}, V_{2}\right)=1$ and $\left|V_{1}\right| \geq\left|V_{2}\right|$. Firstly, assume $1 \notin C\left(V_{1}\right)$. Then $F\left[V_{1}\right]$ is a Gallai$(k-1)$-coloring. Note that $\left|V_{1}\right| \geq|V(F)| / 2 \geq\left(5^{k / 2}+1\right) / 2>2 \cdot 5^{(k-2) / 2}+2$. Since $k$ is even, we have $g r_{k-1}\left(K_{3}: K_{3}\right)=2 \cdot 5^{(k-2) / 2}+1$. Thus there is a monochromatic copy of $K_{3}$ in $F\left[V_{1}\right]$. Let $v$ be a vertex of this $K_{3}$. Since 
$\left|V_{1} \backslash\{v\}\right| \geq 2 \cdot 5^{(k-2) / 2}+1$, there is a monochromatic copy of $K_{3}$ in $F\left[V_{1} \backslash\{v\}\right]$. So there exist two monochromatic copies of $K_{3}$ in $F\left[V_{1}\right]$, a contradiction. We conclude that $1 \in C\left(V_{1}\right)$. In order to avoid two monochromatic copies of $K_{3}$, we have $\left|V_{2}\right|=1$ and there is at most one edge with color 1 in $F\left[V_{1}\right]$. Thus there is a Gallai- $(k-1)$-coloring of $K_{\left|V_{1}\right|-1}$. Since $\left|V_{1}\right|-1 \geq g r_{k-1}\left(K_{3}\right.$ : $K_{3}$ ), there is a monochromatic copy of $K_{3}$ in $F\left[V_{1}\right]$. Then there exist two monochromatic copies of $K_{3}$ in $F$, another contradiction. This solves the case $2 \leq t \leq 3$.

If $t=4$, then we first suppose that $R$ contains a monochromatic copy of $K_{3}$, say $c\left(v_{1} v_{2}\right)=c\left(v_{2} v_{3}\right)=c\left(v_{3} v_{1}\right)=1$. Let $V^{\prime}=V_{1} \cup V_{2} \cup V_{3}$. If $c\left(V_{4}, V^{\prime}\right)=2$, then $V_{4}$ and $V^{\prime}$ form a Gallai partition with exactly two parts, contradicting the minimality of $t$. Thus $c\left(V_{4}, V_{i}\right)=1$ for some $i \in\{1,2,3\}$. But then $c\left(V_{i}, V(G) \backslash V_{i}\right)=1$, contradicting the minimality of $t$. Therefore, $R$ is one of the two 2-edge-colorings of $K_{4}$ without a monochromatic copy of $K_{3}$. In both cases we can derive that there is at most one edge with color 1 or 2 in $\bigcup_{j=1}^{4} F\left[V_{j}\right]$. Thus $|V(F)| \leq 4\left(g r_{k-2}\left(K_{3}: K_{3}\right)-1\right)+1<g r_{k}\left(K_{3}: K_{3}\right)$ by the induction hypothesis, a contradiction.

The remaining case is $t=5$. In this case, there is no edge with color 1 or 2 in $\bigcup_{j=1}^{5} F\left[V_{j}\right]$; otherwise $F$ contains a 2-edge-coloring of $K_{6}$ which contains at least two monochromatic copies of $K_{3}$. By the induction hypothesis, we have $|V(F)| \leq 5\left(g r_{k-2}\left(K_{3}: K_{3}\right)-1\right)<g r_{k}\left(K_{3}: K_{3}\right)$, a contradiction. This completes the proof of Theorem 4.11.

Now we have all the ingredients to present our proof for the lower bound in Theorem 4.4. Let $s_{0}=1$ if $k$ is odd, and $s_{0}=2$ if $k$ is even. By Theorem 4.11, we have $g_{k}\left(K_{3}, g r_{k}\left(K_{3}: K_{3}\right)\right)=s_{0}$. This implies that if $v_{1}, v_{2}, \ldots, v_{g r_{k}}\left(K_{3}: K_{3}\right)$ are any $g r_{k}\left(K_{3}: K_{3}\right)$ vertices of $K_{n}$, then $K_{n}\left[\left\{v_{1}, v_{2}, \ldots, v_{g r_{k}\left(K_{3}: K_{3}\right)}\right\}\right]$ contains at least $s_{0}$ monochromatic copies of $K_{3}$. Since each monochromatic copy of $K_{3}$ is contained in $\left(\begin{array}{c}n-3 \\ g r_{k}\left(K_{3}: K_{3}\right)-3\end{array}\right)$ distinct copies of $K_{g r_{k}\left(K_{3}: K_{3}\right)}$, there are at least

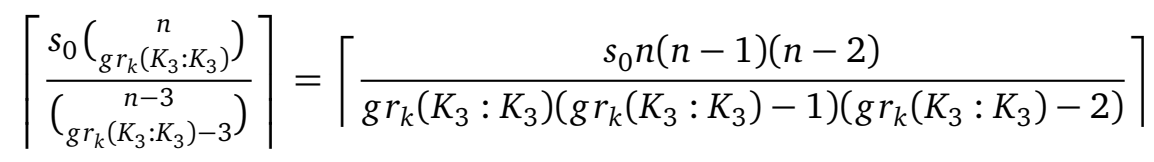


monochromatic copies of $K_{3}$ in any Gallai-k-coloring of $K_{n}$. This completes the proof of Theorem 4.4.

We obtain the following corollary.

Corollary 4.12. Let $k$ be an odd integer. If $0 \leq t \leq 5^{(k-1) / 2}-1$, then $g_{k}\left(K_{3}, g r_{k}\left(K_{3}: K_{3}\right)+t\right)=t+1$.

Proof. The upper bound follows from Theorem 4.4. For the proof of the lower bound, we will use induction on $t$. The case $t=0$ follows from Theorem 4.11. We may assume that $g_{k}\left(K_{3}, g r_{k}\left(K_{3}: K_{3}\right)+(t-1)\right)=(t-1)+1=t$ holds and we will prove it for $t\left(1 \leq t \leq 5^{(k-1) / 2}-1\right)$. Let $n=g r_{k}\left(K_{3}: K_{3}\right)+t$. Note that each monochromatic copy of $K_{3}$ is contained in $\left(\begin{array}{c}n-3 \\ n-1-3\end{array}\right)=n-3$ distinct copies of $K_{n-1}$, and there are $\left(\begin{array}{c}n \\ n-1\end{array}\right)=n$ distinct copies of $K_{n-1}$ in $K_{n}$. By the induction hypothesis, there are at least $\lceil t n /(n-3)\rceil=t+1$ monochromatic copies of $K_{3}$ in any Gallai- $k$-coloring of $K_{n}$.

\subsection{Concluding remarks}

In Section 4.3, we studied the maximum number (denoted by $f_{k}(n, H)$ ) of edges that are not contained in any rainbow triangle or monochromatic copy of $H$. There we showed that $f_{k}(n, H) \geq t\left(n, G R_{k-1}(\mathscr{H})-1\right)$, where $\mathscr{H}$ is the set of homomorphic copies of $H$. Let $f_{k}^{\prime}(n, H)$ be the maximum number of edges not contained in any monochromatic copy of $H$ over all Gallai- $k$-colorings of $K_{n}$. Then we clearly have $f_{k}^{\prime}(n, H) \leq f_{k}(n, H)$. Using the sharpness example constructed in the proof of Lemma 4.8 (2), we can also show that $f_{k}^{\prime}(n, H) \geq t\left(n, G R_{k-1}(\mathscr{H})-1\right)$. Thus we have $t\left(n, G R_{k-1}(\mathscr{H})-\right.$ $1) \leq f_{k}^{\prime}(n, H) \leq f_{k}(n, H)$. An interesting and natural question is for which graphs $H$ the equality $f_{k}^{\prime}(n, H)=f_{k}(n, H)$ holds.

Another problem related to Section 4.3 is to determine the maximum number $\operatorname{nim}_{k}(n, H)$ of edges not contained in any monochromatic copy of $H$ over all $k$-edge-colorings of $K_{n}$. As remarked in [128], if the ErdősSós Conjecture holds for a tree $T$ (i.e., $\operatorname{ex}(n, T) \leq(|V(T)|-2) n / 2)$, then $\operatorname{nim}_{k}(n, T) \geq(k-1) \operatorname{ex}(n, T)$ for $n \geq k^{2}(|V(T)|-1)^{2}$ with $(|V(T)|-1) \mid n$. 
In fact, when $T$ is a star, we can prove the above statement for all integers $n \geq k^{2}(|V(T)|-1)^{2}$.

Let $H$ be an $n$-vertex $K_{1, h}$-free graph with $\operatorname{ex}\left(n, K_{1, h}\right)$ edges. Note that the maximum degree of $H$ is at most $h-1$. For every $i \in[k-1]$, let $f_{i}: V(H) \rightarrow[n]$ be an arbitrary bijection and let $H_{i}$ be the graph obtained by mapping $H$ on $[n]$ via $f_{i}$. Let $H^{*}$ be the graph with vertex set $[n]$ and edge set $\bigcup_{i \in[k-1]} E\left(H_{i}\right)$. Note that $\Delta\left(H^{*}\right) \leq(k-1)(h-1)$. For any vertex $u$, there is a vertex $v$ that is at distance at least three from $u$ in $H^{*}$ since $n>\Delta\left(H^{*}\right)^{2}+1$. If there is an edge $e$ incident with $u$ or $v$ such that $e \in E\left(H_{i}\right) \cap E\left(H_{j}\right)$ for some $1 \leq i \neq j \leq k-1$, then after switching $u$ and $v$ in $f_{i}$, we claim that there is no edge $e^{\prime}$ incident with $u$ or $v$ satisfying $e^{\prime} \in E\left(H_{i}\right) \cap E\left(H_{\ell}\right)$ for any $\ell \in[k-1] \backslash\{i\}$. Otherwise, suppose that there is an edge $v w \in E\left(H_{i}\right) \cap E\left(H_{\ell}\right)$ after switching $u$ and $v$ in $f_{i}$. This implies that before switching $u$ and $v$ in $f_{i}$, we have $v w \in E\left(H_{\ell}\right)$ and $u w \in E\left(H_{i}\right)$. Thus $u w v$ is a path of length two in $H^{*}$, contradicting the fact that $v$ is at distance at least three from $u$. Thus we can repeat this process to obtain a graph with no edge $e$ such that $e \in E\left(H_{i}\right) \cap E\left(H_{j}\right)$ for some $1 \leq i \neq j \leq k-1$. Hence, we can color $K_{n}$ with $c(e)=i$ if $e \in E\left(H_{i}\right)$ for each $i \in[k-1]$ and $c(e)=k$ otherwise. Thus $\operatorname{nim}_{k}\left(n, K_{1, h}\right) \geq \sum_{i \in[k-1]}\left|E\left(H_{i}\right)\right|=(k-1) \operatorname{ex}\left(n, K_{1, h}\right)$.

Moreover, let $G$ be a $k$-edge-coloring of $K_{n}$ with $\operatorname{nim}_{k}\left(n, K_{1, h}\right)$ edges not contained in any monochromatic copy of $K_{1, h}$. For $i \in[k]$, let $G_{i}$ (resp., $G_{i}^{\text {nim }}$ ) denote the spanning subgraph of $G$ with edge set $E\left(G_{i}\right)=\{e \in E(G): c(e)=i\}$ (resp., $E\left(G_{i}^{\text {nim }}\right)=\{e \in E(G): e$ is not contained in any monochromatic copy of $\left.\left.K_{1, h}, c(e)=i\right\}\right)$ and let $V_{i}=\left\{v \in V(G): d_{G_{i}}(v) \geq h\right\}$. If $n>k(h-1)+1$, then $\bigcup_{i \in[k]} V_{i}=V(G)$, and every vertex of $V_{i}$ is an isolated vertex in $G_{i}^{\text {nim }}$ for every $i \in[k]$. Since $\operatorname{ex}\left(n, K_{1, h}\right)=\lfloor(h-1) n / 2\rfloor$, we have $\operatorname{nim}_{k}\left(n, K_{1, h}\right)=$ $\sum_{i \in[k]} e\left(G_{i}^{n i m}\right) \leq \sum_{i \in[k]} e x\left(n-\left|V_{i}\right|, K_{1, h}\right) \leq \operatorname{ex}\left(\sum_{i \in[k]}\left(n-\left|V_{i}\right|\right), K_{1, h}\right) \leq$ $\operatorname{ex}\left((k-1) n, K_{1, h}\right)$. Note that $\operatorname{ex}\left((k-1) n, K_{1, h}\right)=(k-1) \operatorname{ex}\left(n, K_{1, h}\right)+\eta$, where $\eta=\lfloor(k-1) / 2\rfloor$ if $h$ is even and $n$ is odd, and $\eta=0$ otherwise. Therefore, for $n \geq k^{2} h^{2}$, if $h$ is even and $n$ is odd, then $(k-1) \operatorname{ex}\left(n, K_{1, h}\right) \leq$ $\operatorname{nim}_{k}\left(n K_{1, h}\right) \leq(k-1) \operatorname{ex}\left(n, K_{1, h}\right)+\lfloor(k-1) / 2\rfloor$, and otherwise, we have $\operatorname{nim}_{k}\left(n, K_{1, h}\right)=(k-1) \operatorname{ex}\left(n, K_{1, h}\right)$. In particular, we have the following result in the case $k=2$, which partly answers a problem of Keevash and Sudakov [107] 
in the special case when $H$ is a star.

Proposition 4.13. For $n$ sufficiently large, we have $\operatorname{nim}_{2}\left(n, K_{1, h}\right)=\operatorname{ex}\left(n, K_{1, h}\right)$.

In Section 4.4, we studied the minimum number of copies of $K_{3}$ over all Gallai-k-colorings of $K_{n}$. Given an arbitrary $k$-edge-coloring $G$ of $K_{n}$, let $r_{k}\left(K_{3}, n\right)$ and $m_{k}(H, n)$ be the number of rainbow triangles and monochromatic copies of $H$ in $G$, respectively. It is interesting to consider the behavior of $r_{k}\left(K_{3}, n\right)+m_{k}(H, n)$. Clearly if $k \leq 2$, then $r_{k}\left(K_{3}, n\right)+m_{k}(H, n)=m_{k}(H, n)$, and if $G$ is rainbow, then $r_{k}\left(K_{3}, n\right)+m_{k}(H, n)=\left(\begin{array}{l}n \\ 3\end{array}\right)$. However, the general behavior of $r_{k}\left(K_{3}, n\right)+m_{k}(H, n)$ seems difficult to determine.

Finally, we pose two conjectures. Note that we have shown that Conjecture 4.1 below holds for the following cases: (1) $k=3$ and $n$ sufficiently large, (2) $k \geq 3$ and $n=g r_{k}\left(K_{3}: K_{3}\right)$, (3) $k$ is odd and $g r_{k}\left(K_{3}: K_{3}\right) \leq n \leq g r_{k}\left(K_{3}\right.$ : $\left.K_{3}\right)+5^{(k-1) / 2}-1$.

Conjecture 4.1. For $n \geq g r_{k}\left(K_{3}: K_{3}\right)$, we write $n=5^{\lfloor(k-1) / 2\rfloor} m+r$, where $m$ and $r$ are nonnegative integers with $0 \leq r \leq 5^{\lfloor(k-1) / 2\rfloor}-1$. Then

$$
g_{k}\left(K_{3}, n\right)= \begin{cases}r\left(\begin{array}{c}
m+1 \\
3
\end{array}\right)+\left(5^{(k-1) / 2}-r\right)\left(\begin{array}{c}
m \\
3
\end{array}\right), & \text { if } k \text { is odd } \\
r M_{2}\left(K_{3}, m+1\right)+\left(5^{(k-2) / 2}-r\right) M_{2}\left(K_{3}, m\right), & \text { if } k \text { is even. }\end{cases}
$$

Conjecture 4.2. If $k \geq 2$, then $f_{k}\left(n, K_{3}\right)=t\left(n, g r_{k-1}\left(K_{3}: K_{3}\right)-1\right)$. 


\section{Chapter 5}

\section{The Erdős-Gyárfás function with respect to Gallai-colorings}

In this chapter, we consider the function $g(n, p, q)$ that is the minimum number of colors needed for a Gallai- $(p, q)$-coloring of $K_{n}$. Using the anti-Ramsey number for $K_{3}$, we show that $g(n, p, q)$ is nontrivial only for $2 \leq q \leq p-1$. We give a general lower bound for this function and we study how this function falls off from being equal to $n-1$ when $q=p-1$ and $p \geq 4$ to being $\Theta(\log n)$ when $q=2$. In particular, for appropriate $p$ and $n$, we prove that $g=n-c$ when $q=p-c$ and $c \in\{1,2\}, g \leq\left\lceil n^{1 / 2}\right\rceil-1$ when $q=\left\lfloor(p-1)^{1 / 2}\right\rfloor$, and $g$ is logarithmic in $n$ when $2 \leq q \leq\left\lceil\log _{2} p\right\rceil$.

\subsection{Introduction}

Recall that the Erdős-Gyárfás function $f(n, p, q)$ is the minimum number of colors in an edge-coloring of $K_{n}$ such that every $K_{p}$ receives at least $q$ colors. In this chapter, we investigate the Erdős-Gyárfás function within the framework of Gallai-colorings. A Gallai-coloring of the complete graph $K_{n}$ is said to be a Gallai- $(p, q)$-coloring if every $K_{p}$ receives at least $q$ distinct colors. We define $g(n, p, q)$ to be the minimum number of colors that are needed for $K_{n}$ to have a Gallai- $(p, q)$-coloring. Clearly, we have $f(n, p, q) \leq g(n, p, q)$ if both 
functions are defined for these values of $n, p$ and $q$.

For studying $g(n, p, q)$ it is convenient to introduce the following function. For $1 \leq q \leq\left(\begin{array}{c}p \\ 2\end{array}\right)$, let $g_{q}^{k}(p)$ be the smallest positive integer $n$ such that every Gallai- $k$-coloring of $K_{n}$ contains a copy of $K_{p}$ receiving at most $q$ distinct colors. Restated, $g_{q}^{k}(p)-1$ is the largest positive integer $n^{\prime}$ such that there is a Gallai- $k$-coloring of $K_{n^{\prime}}$ in which every $K_{p}$ receives at least $q+1$ distinct colors, i.e., such that $g\left(n^{\prime}, p, q+1\right) \leq k$. Moreover, the following relationship holds:

$$
\left.g_{q-1}^{k}(p) \leq n \Leftrightarrow g(n, p, q)>k \text { (i.e., } g_{q-1}^{k}(p)>n \Leftrightarrow g(n, p, q) \leq k\right) .
$$

Throughout the remainder of the chapter, we concentrate on the function $g_{q}^{k}(p)$ and derive upper and lower bounds and some exact values for this function. It is worth noting that Erdös introduced an analogue of the function $g_{q}^{k}(p)$ when he posed the problem on $f(n, p, q)$ in his original paper [58]. Notice that $g_{1}^{k}(p)=g r_{k}\left(K_{3}: K_{p}\right)$.

We first point out that $g_{q}^{k}(p)$ is nontrivial only for $1 \leq q \leq p-2$ (equivalently, $g(n, p, q)$ is nontrivial only for $2 \leq q \leq p-1)$. When $q \geq p-1$, we can deduce $g_{q}^{k}(p)$ using the following anti-Ramsey result.

Theorem 5.1 (Erdős, Simonovits and Sós [67], Gyárfás and Simonyi [92]). At most $p-1$ colors can be used in any Gallai-coloring of $K_{p}$.

Corollary 5.2. For integers $k \geq 1, p \geq 3$ and $q \geq p-1$, there is no Gallai- $k$ coloring of $K_{n}$ in which every $K_{p}$ receives at least $q+1$ distinct colors. Thus $g_{q}^{k}(p)=p$ for $q \geq p-1$.

Moreover, if $k<q$, then it is obvious that $g_{q}^{k}(p)=p$. In the sequel, we will always assume that $k \geq q$ and $1 \leq q \leq p-2$ when we consider $g_{q}^{k}(p)$. It is easy to verify the following inequalities:

$$
g_{q}^{k}(p) \leq g_{q}^{k+1}(p), \quad g_{q+1}^{k}(p) \leq g_{q}^{k}(p) \text { and } g_{q+1}^{k+1}(p) \leq g_{q}^{k}(p) .
$$

In [75], Fox, Grinshpun and Pach proved the following asymptotic result. Note that for $k=3$ and $q=2$, this result is a special case of the multicolor generalization of the well-known Erdős-Hajnal Conjecture. 
Theorem 5.3 (Fox, Grinshpun and Pach [75]). Let $k$ and $q$ be fixed positive integers with $q \leq k$. Every Gallai-k-coloring of $K_{n}$ contains a set of order $\Omega\left(n\left(\begin{array}{l}q \\ 2\end{array}\right) /\left(\begin{array}{l}k \\ 2\end{array}\right) \log _{2}^{c_{k, q}} n\right)$ which uses at most $q$ colors, where $c_{k, q}$ only depends on $k$ and $q$. Moreover, this bound is tight apart from the constant factor.

It is worth noticing that the problem studied by Fox, Grinshpun and Pach is to find the largest subgraph $K_{p}$ using at most $q$ colors in every Gallai- $k$ coloring of $K_{n}$, for fixed $k$ and $q$, when $n$ is sufficiently large. But in this chapter, we mainly focus on the problem to determine the smallest $n$ such that there is a $K_{p}$ using at most $q$ colors in every Gallai-k-coloring of $K_{n}$, for fixed $p$ and $q$, when $k \in[1,+\infty$ ) (or $k \rightarrow \infty$ ). Therefore, the above theorem cannot give us much support, since it requires that $n$ is sufficiently large, in fact,

$$
n \geq n_{0}=2^{2^{2^{2^{8 k^{2}}}}} .
$$

But we can prove an upper bound of $2^{2 k(p-2) / q+1}$ on $g_{q}^{k}(p)$ (see Theorem 5.4 below). If $2^{2 k(p-2) / q+1} \geq n_{0}$, then $k=o(p)$, which implies that for fixed $p$ and $q$, only $o(p) g_{q}^{k}(p)$ 's can be bounded using the above theorem.

Theorem 5.4. For integers $p, q, k$ with $p \geq 3,1 \leq q \leq p-2$ and $k \geq q$, we have $g_{q}^{k}(p) \leq 2^{2 k(p-2) / q+1}$.

We postpone all proofs of our results to later sections. Note that Theorem 5.4 implies that $g(n, p, q)>\frac{q-1}{2(p-2)}\left(\log _{2} n-1\right)$, where $p \geq 3,2 \leq q \leq p-1$ and $n \geq 2^{2 p-3}$. In [62], Erdős and Gyárfás obtained an upper bound for $f(n, p, q)$ using the Lovász Local Lemma. However, it seems difficult to determine a nontrivial general upper bound for $g(n, p, q)$ (or, equivalently, lower bound for $g_{q}^{k}(p)$ ). Although we can prove some nontrivial results (see, for example Proposition 5.5 below) using the Local Lemma, it cannot help us much in determining an upper bound for $g(n, p, q)$. A graph with an edge-coloring is called $q$-colored if its edges are colored with at most $q$ distinct colors.

Proposition 5.5. For fixed integers $s, q, k$, and appropriately large integer $p$ with $s \geq 4$ and $k \geq \max \left\{\left(\begin{array}{c}s \\ 2\end{array}\right), 2 q+1\right\}$, there exists a k-edge-coloring of $K_{n}$ 
containing neither a rainbow $K_{s}$ nor a q-colored $K_{p}$ with

$$
n=\left(\frac{(s-2) p L^{1 /\left(1-\left(\begin{array}{c}
s \\
2
\end{array}\right)\right)}}{(c+o(1))\left(\left(\begin{array}{l}
s \\
2
\end{array}\right)-2.1\right) \ln \left(p L^{1 /\left(1-\left(\begin{array}{c}
s \\
2
\end{array}\right)\right)}\right)}\right)^{\left(\left(\begin{array}{l}
s \\
2
\end{array}\right)-2.1\right) /(s-2)},
$$

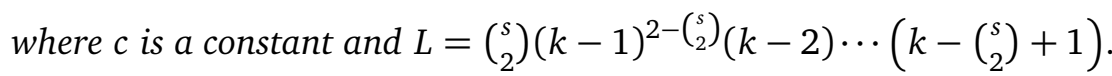

We can slightly improve Theorem 5.4 for $q=1$ by proving the following upper bound on $g_{1}^{k}(p)$.

Theorem 5.6. For integers $k \geq 3$ and $p \geq 5$, we have $g_{1}^{k}(p)=g r_{k}\left(K_{3}: K_{p}\right)<$ $2^{2 k(p-2)-3}$.

When $q=p-2$, we can prove the following result, thereby improving some results obtained in [18].

Theorem 5.7. For integers $p \geq 4$ and $k \geq p-2$, we have $g_{p-2}^{k}(p)=k+2$.

The above result is equivalent to $g(n, p, p-1)=n-1$, where $n \geq p \geq 4$. Using Theorem 5.7, we can show that $g_{q}^{k}(p)$ is at least quadratic in $k$ for $q=\left\lfloor(p-1)^{1 / 2}\right\rfloor-1$.

Theorem 5.8. For integers $p \geq 17$ and $k \geq q=\left\lfloor(p-1)^{1 / 2}\right\rfloor-1$, we have $g_{q}^{k}(p) \geq k^{2}+2 k+2$.

Note that Theorem 5.8 implies that $g\left(n, p,\left\lfloor(p-1)^{1 / 2}\right\rfloor\right) \leq\left\lceil n^{1 / 2}\right\rceil-1$ for $p \geq 17$ and $n$ large enough. When $q=p-3$, we can prove the following result, which is equivalent to $g(n, p, p-2)=n-2$ for $n \geq p \geq 8$.

Theorem 5.9. For integers $p \geq 8$ and $k \geq p-3$, we have $g_{p-3}^{k}(p)=k+3$.

Furthermore, we can determine the exact value of $g_{2}^{k}(5)$. Using this result, we can show that $g_{q}^{k}(p)$ is exponential in $k$ for all $1 \leq q \leq\left\lfloor\log _{2}(p-1)\right\rfloor$.

Theorem 5.10. For integers $k \geq 2$, we have $g_{2}^{k}(5)=2^{k}+1$.

Theorem 5.11. For integers $p \geq 5$ and $k \geq q=\left\lfloor\log _{2}(p-1)\right\rfloor$, we have $g_{q}^{k}(p) \geq 2^{k}+1$. 
Note that Theorem 5.10 implies that $g(n, 5,3)=\left\lceil\log _{2} n\right\rceil$, where $n \geq 5$. Theorem 5.11 implies that $g\left(n, p,\left\lceil\log _{2} p\right\rceil\right) \leq\left\lceil\log _{2} n\right\rceil$ for $p \geq 5$ and $n$ large enough.

Erdős, Hajnal and Rado [64] studied the minimum integer $n$ such that for any $k$-edge-coloring of $K_{n}$ there is a $(k-1)$ )-colored $K_{m}$ (see Section 18 of [64]). Motivated by this problem, we study $g_{k-1}^{k}(p)$ for $k \leq p-1$. If $p$ is sufficiently larger than $k$, then $g_{k-1}^{k}(p)=O\left(\left(p / \log _{2}^{c} p\right)^{k /(k-2)}\right)$ by Theorem 5.3. So we will focus on the case $k / p \rightarrow 1$. By Theorems 5.7 and 5.9, we have $g_{k-1}^{k}(p)=p+1$ for $k \in\{p-1, p-2\}$ and large enough $p$. A natural question is whether $g_{k-1}^{k}(p)=p+1$ for $k=p-c$, where $c$ is a constant and $p$ is large enough. The following theorem answers this question.

Theorem 5.12. For integers $c$, $p$ and $k$ with $c \geq 1, p \geq 2(8+c)^{c+1}-1$ and $k=p-c$, we have $g_{k-1}^{k}(p)=p+1$.

\subsection{Preliminaries}

Since every 2-edge-coloring of $K_{n}$ contains a connected monochromatic spanning subgraph, we have the following corollary by Theorem 1.4.

Corollary 5.13. In any Gallai-coloring of a complete graph, there is a connected monochromatic spanning subgraph.

We shall also use the following simple result in our proofs.

Lemma 5.14. Let $G$ be a Gallai-coloring of a complete graph, $V \subset V(G)$ and $v \in V(G) \backslash V$. Then there is at most one color on the edges between $v$ and $V$ that is not used on any edge within $V$ (that is, $|C(v, V) \backslash C(V)| \leq 1)$.

Proof. Suppose that $c(v u)=1, c(v w)=2$ and $1,2 \notin C(V)$, where $u, w \in V$. Then we may further assume that $c(u w)=3$. Now $\{u, v, w\}$ forms a rainbow triangle, a contradiction.

A covering of a graph $G$ is a subset $V \subseteq V(G)$ such that every edge of $G$ has at least one end-vertex in $V$. 
Theorem 5.15 (The König-Egerváry Theorem, König [109], Egerváry [53]). In any bipartite graph, the number of vertices in a minimum covering is the same as the number of edges in a maximum matching.

Finally, we introduce the Lovász Local Lemma. Let $(\Omega, \mathscr{F}, \operatorname{Pr})$ be a probability space and let $A_{1}, A_{2}, \ldots, A_{n}$ be events. A graph $D$ with $V(D)=\left\{v_{1}, v_{2}\right.$, $\left.\ldots, v_{n}\right\}$ is called a dependency graph for events $A_{1}, A_{2}, \ldots, A_{n}$ if for every $i$, the event $A_{i}$ is mutually independent of all $A_{j}$ with $v_{i} v_{j} \notin E(D)$ and $i \neq j$, i.e., $A_{i}$ is independent of any Boolean function of the events in $\left\{A_{j}: v_{i} v_{j} \notin E(D), i \neq j\right\}$. We shall use the following form of the Local Lemma due to Spencer.

Lemma 5.16 (The Lovász Local Lemma, Erdős and Lovász [66], Spencer [166]). Let $A_{1}, A_{2}, \ldots, A_{n}$ be events in a probability space $(\Omega, \mathscr{F}, \operatorname{Pr})$ with dependency graph $D$. If there exist positive real numbers $y_{1}, y_{2}, \ldots, y_{n}$ such that for each $i, y_{i} \operatorname{Pr}\left(A_{i}\right)<1$ and $\ln y_{i}>\sum_{v_{i} v_{j} \in E(D)} y_{j} \operatorname{Pr}\left(A_{j}\right)$, then $\operatorname{Pr}\left(\wedge_{i=1}^{n} \bar{A}_{i}\right)>0$.

\subsection{General upper and lower bounds}

Before proving Theorem 5.4, we first prove two lemmas. The proof ideas of Lemmas 5.17 and 5.18 below are from [77]. For an edge-colored $K_{n}$, a vertex $v \in V\left(K_{n}\right)$ and a color $i$, let $d_{i}(v)$ be the number of edges in color $i$ incident with $v$.

Lemma 5.17. If an edge-coloring of $K_{n}$ with $n \geq 4$ satisfies $d_{i}(v) \leq \frac{n}{4}$ for each $v \in V\left(K_{n}\right)$ and each color $i$, then there exists a rainbow copy of $K_{3}$.

Proof. It suffices to show that the number of non-rainbow $K_{3}$ 's is less than $\left(\begin{array}{l}n \\ 3\end{array}\right)$. Note that for any vertex $v$ and any color $i$, there are at most $\left(\begin{array}{c}d_{i}(v) \\ 2\end{array}\right)$ non-rainbow $K_{3}$ 's with two edges in color $i$ incident with vertex $v$. Thus the number of non-rainbow $K_{3}$ 's is at most

$$
\sum_{v \in V\left(K_{n}\right)} \sum_{i} \frac{d_{i}(v)\left(d_{i}(v)-1\right)}{2} \leq 4 n \frac{(n / 4)(n / 4-1)}{2}<\left(\begin{array}{l}
n \\
3
\end{array}\right),
$$


where the first inequality holds since $\sum_{i} \frac{d_{i}(v)\left(d_{i}(v)-1\right)}{2} \leq 4 \frac{(n / 4)(n / 4-1)}{2}$ (using $0 \leq d_{i}(v) \leq \frac{n}{4}, \sum_{i} d_{i}(v)=n-1$, and noting that the function $f(x)=\frac{x(x-1)}{2}$ is convex with $f(x) \geq f(1)=0$ for any $x \geq 1)$.

Let $[k]$ be a set of colors and $t_{q}=\sum_{i=1}^{q}\left(\begin{array}{l}k \\ i\end{array}\right)$. Let $\mathscr{I}=\{I \subseteq[k]: 1 \leq|I| \leq$ $q\}=\left\{I_{1}, I_{2}, \ldots, I_{t_{q}}\right\}$. Then we define $g_{q}^{k}\left(p_{1}, p_{2}, \ldots, p_{t_{q}}\right)$ to be the smallest positive integer $n$ such that every Gallai- $k$-coloring of $K_{n}$ contains a copy of $K_{p_{i}}$ all edges of which have colors from one set $I_{i}$ for some $i \in\left[t_{q}\right]$.

Lemma 5.18. We have

$$
g_{q}^{k}\left(p_{1}, p_{2}, \ldots, p_{t_{q}}\right) \leq 4 \cdot \max _{1 \leq i \leq k} g_{q}^{k}\left(p_{1}^{(i)}, p_{2}^{(i)}, \ldots, p_{t_{q}}^{(i)}\right),
$$

where $p_{j}^{(i)}=p_{j}-1$ if $i \in I_{j}$, and $p_{j}^{(i)}=p_{j}$ otherwise.

Proof. Let $n \geq 4 \cdot \max _{1 \leq i \leq k} g_{q}^{k}\left(p_{1}^{(i)}, p_{2}^{(i)}, \ldots, p_{t_{q}}^{(i)}\right)$. By Lemma 5.17, for every Gallai-coloring of $K_{n}$, there exists a vertex $v$ and a color $\ell$ with $d_{\ell}(v)>\frac{n}{4}$. Let $N_{\ell}(v)=\{u: c(u v)=\ell\}$. Then $\left|N_{\ell}(v)\right|>g_{q}^{k}\left(p_{1}^{(\ell)}, p_{2}^{(\ell)}, \ldots, p_{t_{q}}^{(\ell)}\right)$. In this case there is a copy of $K_{p_{i}}$ all edges of which have colors from one set $I_{i}$ for some $i$. This proves the statement of the lemma.

Now we have all ingredients to present our proofs of Theorems 5.4 and 5.6 .

Proof of Theorem 5.4. Note that $g_{q}^{k}(p)=g_{q}^{k}(p, p, \ldots, p)$. We can repeatedly apply Lemma 5.18 until in some step we get $g_{q}^{k}\left(p_{1}, p_{2}, \ldots, p_{t_{q}}\right) \leq 2$. In each step, we have $g_{q}^{k}\left(p_{1}, p_{2}, \ldots, p_{t_{q}}\right) \leq 4 \cdot g_{q}^{k}\left(p_{1}^{(i)}, p_{2}^{(i)}, \ldots, p_{t_{q}}^{(i)}\right)$ for some $i$. For each $i \in[k]$, let $\alpha(i)$ be the number of steps in which we apply Lemma 5.18 for color $i$. By the definition of $g_{q}^{k}\left(p_{1}, p_{2}, \ldots, p_{t_{q}}\right)$, we have $g_{q}^{k}\left(p_{1}, p_{2}, \ldots, p_{t_{q}}\right)=$ $1<2$ if $p_{j}=1$ for some $j \in\left[t_{q}\right]$. We also have $g_{q}^{k}\left(p_{1}, p_{2}, \ldots, p_{t_{q}}\right)=2$ if $p_{j}=2$ for all $j \in\left[t_{q}\right]$ with $\left|I_{j}\right|=q$. Thus we have $\sum_{I \in \mathscr{I},|I|=q} \sum_{i \in I} \alpha(i) \leq(p-$ 2) $\left(\begin{array}{l}k \\ q\end{array}\right)$. So $\sum_{i=1}^{k} \alpha(i)=\frac{1}{\left(\begin{array}{c}k-1 \\ q-1\end{array}\right)} \sum_{I \in \mathscr{I},|I|=q} \sum_{i \in I} \alpha(i) \leq \frac{1}{\left(\begin{array}{c}k-1 \\ q-1\end{array}\right)}\left(\begin{array}{l}k \\ q\end{array}\right)(p-2)=\frac{k(p-2)}{q}$. We conclude that $g_{q}^{k}(p) \leq 4^{\frac{k(p-2)}{q}} \cdot 2=2^{\frac{2 k(p-2)}{q}+1}$, completing the proof of Theorem 5.4. 
Proof of Theorem 5.6. The proof is similar to the proof of Theorem 5.4. The only difference is that we repeatedly apply Lemma 5.18 until in some step we get $g_{1}^{k}\left(p_{1}, p_{2}, \ldots, p_{t_{q}}\right)<32$. Note that $g_{1}^{k}(2)=2 \leq 32, g_{1}^{k}(2, \ldots, 2$, 6) $=6<32, g_{1}^{k}(2, \ldots, 2,3,5)=r(3,5)=14<32$ (see [88]), $g_{1}^{k}(2, \ldots, 2,4$, $4)=r_{2}\left(K_{4}\right)=18<32$ (see [88]), $g_{1}^{k}(2, \ldots, 2,3,3,4)=g_{1}^{3}(3,3,4)=17<32$ (see [123]) and $g_{1}^{k}(2, \ldots, 2,3,3,3,3)=g_{1}^{4}(3)=26<32$ (see Theorem 2.7). Thus we have $\sum_{i=1}^{k} \alpha(i) \leq k(p-2)-4$ in this case, so $g_{1}^{k}(p)<4^{k(p-2)-4} \cdot 32=$ $2^{2 k(p-2)-3}$.

In the rest of this section, we prove Proposition 5.5, using a similar method to that used in [171].

Proof of Proposition 5.5. Consider a $k$-edge-coloring $G$ of $K_{n}$, where each edge receives color $i(1 \leq i \leq k-1)$ with probability $\frac{r}{k-1}$ and color $k$ with probability $1-r$ (for small $r$, to be determined shortly), and these probabilities are mutually independent. For each set $S$ of $s$ vertices, let $A_{S}$ be the event that $G[S]$ is a rainbow $K_{s}$. For each set $T$ of $p$ vertices, let $B_{T}$ be the event that $G[T]$ is a $q$-colored $K_{p}$. We shall show that $\operatorname{Pr}\left(\left(\wedge_{S} \bar{A}_{S}\right) \wedge\left(\wedge_{T} \bar{B}_{T}\right)\right)>0$.

Define a graph $D$ with a vertex set corresponding to all possible $A_{S}$ and $B_{T}$ such that (the vertex corresponding to) $A_{S}$ is adjacent to (the vertex corresponding to) $B_{T}$ if and only if $|S \cap T| \geq 2$, and $A_{S}$ (resp., $B_{T}$ ) is adjacent to $A_{S^{\prime}}$ (resp., $B_{T^{\prime}}$ ) if and only if $\left|S \cap S^{\prime}\right| \geq 2$ (resp., $\left|T \cap T^{\prime}\right| \geq 2$ ). Then $D$ is a dependency graph. We define $N_{A A}, N_{A B}, N_{B A}$ and $N_{B B}$ such that $N_{X Y}$ is the number of vertices in $D$ of type $Y$ (so corresponding either to a number of $A_{S}$ vertices or a number of $B_{T}$ vertices) adjacent to a fixed vertex of type $X$ (so either one $A_{S}$ vertex or one $B_{T}$ vertex). In order to be able to apply Lemma 5.16, for each $S$, let the positive real number $y_{i}=y$ correspond to event $A_{S}$, and for each $T$, let $y_{i}=z$ correspond to event $B_{T}$. By Lemma 5.16, to show that $\operatorname{Pr}\left(\left(\wedge_{S} \bar{A}_{S}\right) \wedge\left(\wedge_{T} \bar{B}_{T}\right)\right)>0$, it suffices to show that there exist positive real numbers $r, y, z$ such that

$$
\begin{aligned}
& r<1, y \operatorname{Pr}\left(A_{s}\right)<1, z \operatorname{Pr}\left(B_{T}\right)<1, \\
& \ln y>y \operatorname{Pr}\left(A_{s}\right) N_{A A}+z \operatorname{Pr}\left(B_{T}\right) N_{A B},
\end{aligned}
$$


and

$$
\ln z>y \operatorname{Pr}\left(A_{s}\right) N_{B A}+z \operatorname{Pr}\left(B_{T}\right) N_{B B} .
$$

Note that for $r$ small, we have

$$
\begin{aligned}
\operatorname{Pr}\left(B_{T}\right) & \leq\left(\begin{array}{c}
k-1 \\
q
\end{array}\right)\left(\frac{q r}{k-1}\right)^{\left(\begin{array}{l}
p \\
2
\end{array}\right)}+\left(\begin{array}{c}
k-1 \\
q-1
\end{array}\right)\left(1-r+\frac{(q-1) r}{k-1}\right)^{\left(\begin{array}{l}
p \\
2
\end{array}\right)} \\
& \leq\left(\begin{array}{c}
k-1 \\
q
\end{array}\right)\left(\frac{r}{2}\right)^{\left(\begin{array}{l}
p \\
2
\end{array}\right)}+\left(\begin{array}{c}
k-1 \\
q-1
\end{array}\right)\left(1-\frac{r}{2}\right)^{\left(\begin{array}{l}
p \\
2
\end{array}\right)} \\
& \leq\left(\left(\begin{array}{c}
k-1 \\
q
\end{array}\right)+\left(\begin{array}{c}
k-1 \\
q-1
\end{array}\right)\right)\left(1-\frac{r}{2}\right)^{\left(\begin{array}{c}
p \\
2
\end{array}\right)} \\
& \leq\left(\begin{array}{c}
k \\
q
\end{array}\right) \exp \left(-\frac{r}{2}\left(\begin{array}{l}
p \\
2
\end{array}\right)\right)=\exp \left(-\frac{r p^{2}}{4}+\frac{r p}{4}+\ln \left(\begin{array}{l}
k \\
q
\end{array}\right)\right)
\end{aligned}
$$

and

$$
\begin{aligned}
\operatorname{Pr}\left(A_{S}\right) \leq & \left(\begin{array}{c}
k-1 \\
\left(\begin{array}{l}
s \\
2
\end{array}\right)
\end{array}\right)\left(\begin{array}{l}
s \\
2
\end{array}\right) !\left(\frac{r}{k-1}\right)^{\left(\begin{array}{l}
s \\
2
\end{array}\right)} \\
& +\left(\begin{array}{c}
k-1 \\
\left(\begin{array}{l}
s \\
2
\end{array}\right)-1
\end{array}\right)\left(\left(\begin{array}{l}
s \\
2
\end{array}\right)-1\right) !\left(\begin{array}{l}
s \\
2
\end{array}\right)(1-r)\left(\frac{r}{k-1}\right)^{\left(\begin{array}{l}
s \\
2
\end{array}\right)-1} \\
= & \left(\begin{array}{l}
s \\
2
\end{array}\right)(k-1)(k-2) \cdots\left(k-\left(\begin{array}{l}
s \\
2
\end{array}\right)+1\right)\left(\frac{r}{k-1}\right)^{\left(\begin{array}{l}
s \\
2
\end{array}\right)-1} \\
& \cdot\left(\frac{k-\left(\begin{array}{l}
s \\
2
\end{array}\right)}{\left(\begin{array}{l}
s \\
2
\end{array}\right)} \cdot \frac{r}{k-1}+1-r\right) \\
\leq & L r^{\left(\begin{array}{l}
s \\
2
\end{array}\right)-1} .
\end{aligned}
$$

We bound $N_{A A}, N_{A B}, N_{B A}$ and $N_{B B}$ as follows:

$$
\begin{gathered}
N_{A A} \leq\left(\begin{array}{l}
s \\
2
\end{array}\right)\left(\begin{array}{l}
n-2 \\
s-2
\end{array}\right) \leq s^{2} n^{s-2}, N_{A B} \leq\left(\begin{array}{l}
s \\
2
\end{array}\right)\left(\begin{array}{l}
n-2 \\
p-2
\end{array}\right) \leq s^{2} n^{p-2}, \\
N_{B A} \leq\left(\begin{array}{l}
p \\
2
\end{array}\right)\left(\begin{array}{l}
n-2 \\
s-2
\end{array}\right) \leq p^{2} n^{s-2}, N_{B B} \leq\left(\begin{array}{l}
p \\
2
\end{array}\right)\left(\begin{array}{l}
n-2 \\
p-2
\end{array}\right) \leq p^{2} n^{p-2} .
\end{gathered}
$$


Let $\alpha=(s-2) /\left(\left(\begin{array}{l}s \\ 2\end{array}\right)-2.1\right)$ and $\beta=1 /\left(\left(\begin{array}{l}s \\ 2\end{array}\right)-1\right)$. We set

$$
r=c_{1} n^{-\alpha} L^{-\beta}, p=c_{2} n^{\alpha}(\ln n) L^{\beta}, y=1+\epsilon, z=\exp \left(c_{3} n^{\alpha}(\ln n)^{2} L^{\beta}\right),
$$

where $c_{1}, c_{2}, c_{3}$ are appropriately chosen, $\epsilon \ll 1$ and $n$ tends to infinity. Then we have

$$
\begin{aligned}
& y \operatorname{Pr}\left(A_{S}\right) N_{A A} \leq(1+\epsilon) L r^{\left(\begin{array}{l}
s \\
2
\end{array}\right)-1} s^{2} n^{s-2}=(1+\epsilon) s^{2} c_{1}^{\left(\begin{array}{l}
s \\
2
\end{array}\right)-1} n^{\frac{-1.1(s-2)}{\left(\begin{array}{l}
s \\
2
\end{array}\right)-2.1}}, \\
& y \operatorname{Pr}\left(A_{S}\right) N_{B A} \leq(1+\epsilon) L r^{\left(\begin{array}{c}
s \\
2
\end{array}\right)-1} p^{2} n^{s-2}=(1+\epsilon) c_{1}^{\left(\begin{array}{c}
s \\
2
\end{array}\right)-1} c_{2}^{2} L^{2 \beta} n^{\alpha-\frac{0.1(s-2)}{\left(\begin{array}{c}
s \\
2
\end{array}\right)-2.1}}(\ln n)^{2}, \\
& z \operatorname{Pr}\left(B_{T}\right) N_{A B} \leq \exp \left(c_{3} n^{\alpha}(\ln n)^{2} L^{\beta}-\frac{r p^{2}}{4}+\frac{r p}{4}\right. \\
& \left.+\ln \left(\begin{array}{l}
k \\
q
\end{array}\right)+2 \ln s+(p-2) \ln n\right) \\
& \leq \exp \left(c_{3} n^{\alpha}(\ln n)^{2} L^{\beta}-\frac{c_{1} c_{2}^{2}}{4} n^{\alpha}(\ln n)^{2} L^{\beta}\right. \\
& \left.+o\left(n^{\alpha}(\ln n)^{2}\right)+c_{2} n^{\alpha}(\ln n)^{2} L^{\beta}\right) \\
& \leq \exp \left(\left(c_{3}-\frac{c_{1} c_{2}^{2}}{4}+c_{2}+o(1)\right) n^{\alpha}(\ln n)^{2} L^{\beta}\right)
\end{aligned}
$$

and

$$
\begin{aligned}
z \operatorname{Pr}\left(B_{T}\right) N_{B B} \leq & \exp \left(c_{3} n^{\alpha}(\ln n)^{2} L^{\beta}-\frac{r p^{2}}{4}+\frac{r p}{4}\right. \\
& \left.+\ln \left(\begin{array}{l}
k \\
q
\end{array}\right)+2 \ln p+(p-2) \ln n\right) \\
\leq & \exp \left(\left(c_{3}-\frac{c_{1} c_{2}^{2}}{4}+c_{2}+o(1)\right) n^{\alpha}(\ln n)^{2} L^{\beta}\right) .
\end{aligned}
$$

If we choose $c_{1}, c_{2}, c_{3}$ such that $c_{3}-\frac{c_{1} c_{2}^{2}}{4}+c_{2}+o(1)<0$, then inequalities (5.1)-(5.3) hold. Setting $c=c_{2}$ in the above expression for $p$, and expressing 
$n$ in terms of $p$, we have

$$
n \geq\left(\frac{(s-2) p L^{1 /\left(1-\left(\begin{array}{c}
s \\
2
\end{array}\right)\right)}}{(c+o(1))\left(\left(\begin{array}{l}
s \\
2
\end{array}\right)-2.1\right) \ln \left(p L^{1 /\left(1-\left(\begin{array}{c}
s \\
2
\end{array}\right)\right)}\right)}\right)^{\left.\left(\begin{array}{l}
s \\
2
\end{array}\right)-2 \cdot 1\right) /(s-2)} .
$$

\subsection{Proofs of Theorems 5.7 and 5.8}

We first present our proof of Theorem 5.7.

Proof of Theorem 5.7. We first show that there is a Gallai- $k$-coloring of $K_{k+1}$, in which there is no $K_{p}$ receiving at most $p-2$ distinct colors. The case $k=p-2$ is trivial since $K_{p-1}$ contains no $K_{p}$. For $k \geq p-1$, let $V\left(K_{k+1}\right)=$ $\left\{v_{1}, v_{2}, \ldots, v_{k+1}\right\}$. For every $1 \leq i<j \leq k+1$, we color the edge $v_{i} v_{j}$ using color $i$. Note that for any three vertices $v_{i}, v_{j}, v_{k}$ with $i<j<k$, we have $c\left(v_{i} v_{j}\right)=c\left(v_{i} v_{k}\right)$, so there is no rainbow triangle. For any $p$ vertices $v_{i_{1}}, v_{i_{2}}, \ldots, v_{i_{p}}$ with $i_{1}<i_{2}<\cdots<i_{p}$, we have $C\left(\left\{v_{i_{1}}, v_{i_{2}}, \ldots, v_{i_{p}}\right\}\right)=$ $\left\{i_{1}, i_{2}, \ldots, i_{p-1}\right\}$, so every $K_{p}$ receives $p-1$ distinct colors.

Next, we show that $g_{p-2}^{k}(p) \leq k+2$ by induction on $k$. For the base case, if $k=p-2$, then it is trivial that $g_{p-2}^{k}(p)=p$. Now assume that it holds for every $p-2 \leq k^{\prime} \leq k-1$, and we will prove it for $k$.

For a contradiction, suppose that $G$ is a Gallai-k-coloring of $K_{k+2}$ without a $(p-2)$-colored $K_{p}$. Using Theorem 1.4, let $V_{1}, V_{2}, \ldots, V_{m}(m \geq 2)$ be a Gallai partition of $V(G)$. Note that $m \leq p-1$ since $p-2 \geq 2$. If $m \geq 4$, then we can choose nonempty subsets $V_{i}^{\prime} \subseteq V_{i}(1 \leq i \leq m)$ such that $\sum_{i=1}^{m}\left|V_{i}^{\prime}\right|=p$. Since $G$ is a Gallai-coloring, we have $\left|C\left(V_{i}^{\prime}\right)\right| \leq\left|V_{i}^{\prime}\right|-1(1 \leq i \leq m)$ by Theorem 5.1. Then $\left|C\left(\bigcup_{i=1}^{m} V_{i}^{\prime}\right)\right| \leq 2+\sum_{i=1}^{m}\left(\left|V_{i}^{\prime}\right|-1\right)=2+p-m \leq p-2$. Thus there is a $(p-2)$-colored $K_{p}$ in $G$, a contradiction. Hence, we have $m \leq 3$. Note that if $G$ contains a Gallai partition with exactly three parts, then $G$ also contains a Gallai partition with exactly two parts. Thus we may assume that $m=2$ and $c\left(V_{1}, V_{2}\right)=1$.

Claim 5.1. $1 \notin C\left(V_{1}\right)$ and $1 \notin C\left(V_{2}\right)$. 
Proof. By symmetry, we only prove $1 \notin C\left(V_{1}\right)$. If $1 \in C\left(V_{1}\right)$, then we may choose $V_{1}^{\prime} \subseteq V_{1}$ and $V_{2}^{\prime} \subseteq V_{2}$ such that $1 \in C\left(V_{1}^{\prime}\right)$ and $\left|V_{1}^{\prime}\right|+\left|V_{2}^{\prime}\right|=p$. Thus $\left|C\left(V_{1}^{\prime} \cup V_{2}^{\prime}\right)\right| \leq\left|C\left(V_{1}^{\prime}\right)\right|+\left|C\left(V_{2}^{\prime}\right)\right| \leq\left|V_{1}^{\prime}\right|-1+\left|V_{2}^{\prime}\right|-1=p-2$, a contradiction.

Claim 5.2. $\left|V_{1}\right|=\left|C\left(V_{1}\right)\right|+1$ and $\left|V_{2}\right|=\left|C\left(V_{2}\right)\right|+1$.

Proof. By symmetry, we only prove it for $V_{1}$. By Theorem 5.1, we have $\left|V_{1}\right| \geq\left|C\left(V_{1}\right)\right|+1$, so it suffices to prove $\left|V_{1}\right| \leq\left|C\left(V_{1}\right)\right|+1$. Suppose for a contradiction that $\left|V_{1}\right| \geq\left|C\left(V_{1}\right)\right|+2$. If $\left|C\left(V_{1}\right)\right| \leq p-3$, then $\left|V_{1}\right| \leq p-1$ in order to avoid a $(p-2)$-colored $K_{p}$. Thus we can choose $V_{2}^{\prime} \subseteq V_{2}$ with $\left|V_{1}\right|+\left|V_{2}^{\prime}\right|=p$. Since $\left|C\left(V_{2}^{\prime}\right)\right| \leq\left|V_{2}^{\prime}\right|-1$, we have $\left|C\left(V_{1} \cup V_{2}^{\prime}\right)\right| \leq 1+\left|C\left(V_{1}\right)\right|+$ $\left|V_{2}^{\prime}\right|-1 \leq\left|C\left(V_{1}\right)\right|+p-\left|V_{1}\right| \leq\left|C\left(V_{1}\right)\right|+p-\left(\left|C\left(V_{1}\right)\right|+2\right)=p-2$, a contradiction. Thus $\left|C\left(V_{1}\right)\right| \geq p-2$, and then we have $\left|V_{1}\right| \leq\left|C\left(V_{1}\right)\right|+1$ by Claim 5.1 and the induction hypothesis.

We now show that $C\left(V_{1}\right) \cap C\left(V_{2}\right)=\emptyset$. Otherwise, suppose $2 \in C\left(V_{1}\right) \cap$ $C\left(V_{2}\right)$. We choose $V_{1}^{\prime} \subseteq V_{1}$ and $V_{2}^{\prime} \subseteq V_{2}$ such that $2 \in C\left(V_{1}^{\prime}\right), 2 \in C\left(V_{2}^{\prime}\right)$ and $\left|V_{1}^{\prime}\right|+\left|V_{2}^{\prime}\right|=p$. Then $\left|C\left(V_{1}^{\prime} \cup V_{2}^{\prime}\right)\right| \leq 1+\left|C\left(V_{1}^{\prime}\right)\right|+\left|C\left(V_{2}^{\prime}\right)\right|-1 \leq\left|V_{1}^{\prime}\right|-1+$ $\left|V_{2}^{\prime}\right|-1=p-2$, a contradiction. Finally, by Claims 5.1 and 5.2 , we have $k+2=|V(G)|=\left|V_{1}\right|+\left|V_{2}\right|=\left|C\left(V_{1}\right)\right|+1+\left|C\left(V_{2}\right)\right|+1 \leq k-1+2=k+1$, a contradiction.

In the following, instead of proving Theorem 5.8, we will prove the following more general result.

Theorem 5.19. For integers $p \gg m \geq 2$ and $k \geq q=\left\lfloor(p-1)^{1 / m}\right\rfloor-1$, we have $g_{q}^{k}(p) \geq(k+1)^{m}+1$.

Proof. By Theorem 5.7, we have $g_{q}^{k}(q+2)>k+1$. Let $G_{0}$ be a Gallai- $k$ coloring of $K_{k+1}$ in which the largest $q$-colored complete subgraph has order at most $q+1$, and let $G_{1}=G_{0}$. Suppose for some $1 \leq i<m$ we have constructed a $k$-edge-coloring $G_{i}$ of $K_{(k+1)}$. Then we construct $G_{i+1}$ such that $G_{i+1}=G_{0}\left((k+1) \cdot G_{i}\right)$. Finally, we obtain a $k$-edge-coloring $G_{m}$ of $K_{(k+1)^{m}}$. It is easy to check that $G_{m}$ is a Gallai-coloring and that the largest $q$-colored complete subgraph has order at most $(q+1)^{m} \leq p-1$. Thus we have $g_{q}^{k}(p) \geq(k+1)^{m}+1$. 


\subsection{Proof of Theorem 5.9}

In this section, we present our proof of Theorem 5.9. For the lower bound, we will construct a Gallai- $k$-coloring of $K_{k+2}$ without a $(p-3)$-colored $K_{p}$. The case $k=p-3$ is trivial since $K_{p-1}$ contains no $K_{p}$. For $k \geq p-2$, let $V\left(K_{k+2}\right)=\left\{v_{1}, v_{2}, \ldots, v_{k+2}\right\}$. For every $1 \leq i \leq k$ and $i<j \leq k+2$, we color the edge $v_{i} v_{j}$ using color $i$, and we color the edge $v_{k+1} v_{k+2}$ with color $k$. Then we obtain a desired edge-coloring.

For the upper bound, we will use induction on $k$. For the base case, if $k=p-3$, then it is trivial that $g_{p-3}^{k}(p) \leq k+3$. Now assume that it holds for every $p-3 \leq k^{\prime} \leq k-1$, and we will prove it for $k$. For a contradiction, suppose that $G$ is a Gallai- $k$-coloring of $K_{k+3}$ without a $(p-3)$-colored $K_{p}$. By the induction hypothesis, we may assume that all the $k$ colors appear in $G$ (that is, $C(G)=[k])$. Using Theorem 1.4, let $V_{1}, V_{2}, \ldots, V_{m}(m \geq 2)$ be a Gallai partition of $V(G)$. We choose it such that $m$ is minimum.

Case 1. $m \geq 4$.

In this case, by the minimality of $m$, there are exactly two colors used between the parts, say colors 1 and 2 . If $m \geq 5$, then we can choose one vertex $v_{i}$ from each $V_{i}(1 \leq i \leq 5)$ to form a 2-colored $K_{5}$. Then we choose another $p-5$ vertices $v_{6}, v_{7}, \ldots, v_{p}$ one by one arbitrarily. Note that for each $6 \leq i \leq p$, when we add vertex $v_{i}$ to $G_{i-1}=G\left[\left\{v_{1}, v_{2}, \ldots, v_{i-1}\right\}\right]$, we add at most one new color that is not used in $G_{i-1}$, by Lemma 5.14. Thus we obtain a $(p-3)$-colored $K_{p}$, a contradiction. Hence, we have $m=4$.

Claim 5.3. For any $i \in[4]$, we have $1,2 \notin C\left(V_{i}\right)$. For any $1 \leq i<j \leq 4$, we have $C\left(V_{i}\right) \cap C\left(V_{j}\right)=\emptyset$.

Proof. If $C\left(V_{i}\right) \cap\{1,2\} \neq \emptyset$ for some $i \in[4]$, then we can choose nonempty subsets $V_{\ell}^{\prime} \subseteq V_{\ell}(1 \leq \ell \leq 4)$ such that $\sum_{\ell=1}^{4}\left|V_{\ell}^{\prime}\right|=p$ and $C\left(V_{i}^{\prime}\right) \cap\{1,2\} \neq \emptyset$. Since $G$ is a Gallai-coloring, we have $\left|C\left(V_{\ell}^{\prime}\right)\right| \leq\left|V_{\ell}^{\prime}\right|-1(1 \leq \ell \leq 4)$ by Theorem 5.1. Then $\left|C\left(\bigcup_{\ell=1}^{4} V_{\ell}^{\prime}\right)\right| \leq 2+\left(\sum_{\ell=1}^{4}\left|C\left(V_{\ell}^{\prime}\right)\right|\right)-1 \leq 2+\left(\sum_{\ell=1}^{4}\left(\left|V_{\ell}^{\prime}\right|-1\right)\right)-1=$ $2+p-4-1=p-3$. Thus there is a $(p-3)$-colored $K_{p}$ in $G$, a contradiction. If $C\left(V_{i}\right) \cap C\left(V_{j}\right) \neq \emptyset$ for some $1 \leq i<j \leq 4$, say $c_{0} \in C\left(V_{i}\right) \cap C\left(V_{j}\right)$, then we can choose nonempty subsets $V_{\ell}^{\prime} \subseteq V_{\ell}(1 \leq \ell \leq 4)$ such that $\sum_{\ell=1}^{4}\left|V_{\ell}^{\prime}\right|=p$, 
$c_{0} \in C\left(V_{i}^{\prime}\right)$ and $c_{0} \in C\left(V_{j}^{\prime}\right)$. Then $\left|C\left(\bigcup_{\ell=1}^{4} V_{\ell}^{\prime}\right)\right| \leq 2+\left(\sum_{\ell=1}^{4}\left|C\left(V_{\ell}^{\prime}\right)\right|\right)-1 \leq$ $2+\left(\sum_{\ell=1}^{4}\left(\left|V_{\ell}^{\prime}\right|-1\right)\right)-1=p-3$. Thus there is a $(p-3)$-colored $K_{p}$ in $G$, a contradiction.

Claim 5.4. For any $i \in[4]$, we have $\left|V_{i}\right| \leq\left|C\left(V_{i}\right)\right|+1$.

Proof. Suppose for a contradiction that $\left|V_{i}\right| \geq\left|C\left(V_{i}\right)\right|+2$ for some $i \in[4]$, say $i=1$. If $\left|C\left(V_{1}\right)\right| \leq p-5$, then $\left|V_{1}\right| \leq p-4$ in order to avoid a $(p-3)$-colored $K_{p}$. Thus we can choose nonempty subsets $V_{j}^{\prime} \subseteq V_{j}(2 \leq j \leq 4)$ such that $\left|V_{1}\right|+\sum_{j=2}^{4}\left|V_{j}^{\prime}\right|=p$. Then $\left|C\left(V_{1} \cup\left(\bigcup_{j=2}^{4} V_{j}^{\prime}\right)\right)\right| \leq 2+\left|C\left(V_{1}\right)\right|+\sum_{j=2}^{4}\left(\left|V_{j}^{\prime}\right|-1\right) \leq$ $2+\left|C\left(V_{1}\right)\right|+\left(p-\left|V_{1}\right|\right)-3 \leq 2+\left|C\left(V_{1}\right)\right|+p-\left(\left|C\left(V_{1}\right)\right|+2\right)-3=p-3$, a contradiction.

If $\left|C\left(V_{1}\right)\right| \geq p-3$, then $\left|V_{1}\right| \leq\left|C\left(V_{1}\right)\right|+2$ by Claim 5.3 and the induction hypothesis. If $\left|C\left(V_{1}\right)\right|=p-4$, then $\left|V_{1}\right| \leq p-2=\left|C\left(V_{1}\right)\right|+2$ in order to avoid a $(p-3)$-colored $K_{p}$. Thus $\left|V_{1}\right|=\left|C\left(V_{1}\right)\right|+2$ whenever $\left|C\left(V_{1}\right)\right| \geq p-4$. By Theorem 5.7, there is a $\left(p_{1}-2\right)$-colored $K_{p_{1}}$ in $G\left[V_{1}\right]$ for every $4 \leq p_{1} \leq\left|V_{1}\right|$. Let $H$ be a copy of a $(p-5)$-colored $K_{p-3}$ in $G\left[V_{1}\right]$. Then we can choose one vertex from each $V_{j}(2 \leq j \leq 4)$ such that these vertices together with $H$ form a $(p-3)$-colored $K_{p}$, a contradiction.

By Claims 5.3 and 5.4, we have $|V(G)|=\sum_{i=1}^{4}\left|V_{i}\right| \leq \sum_{i=1}^{4}\left(\left|C\left(V_{i}\right)\right|+1\right) \leq$ $k-2+4=k+2<k+3$, a contradiction.

Case 2. $2 \leq m \leq 3$.

By the minimality of $m$, we may assume that $m=2$ and $c\left(V_{1}, V_{2}\right)=1$.

Claim 5.5. At most one of $V_{1}$ and $V_{2}$ contains an edge with color 1 .

Proof. If $1 \in C\left(V_{1}\right)$ and $1 \in C\left(V_{2}\right)$, then we can choose $V_{1}^{\prime} \subseteq V_{1}$ and $V_{2}^{\prime} \subseteq V_{2}$ such that $\left|V_{1}^{\prime}\right|+\left|V_{2}^{\prime}\right|=p, 1 \in C\left(V_{1}^{\prime}\right)$ and $1 \in C\left(V_{2}^{\prime}\right)$. Then $\left|C\left(V_{1}^{\prime} \cup V_{2}^{\prime}\right)\right| \leq$ $\left|C\left(V_{1}^{\prime}\right)\right|+\left|C\left(V_{2}^{\prime}\right)\right|-1 \leq\left|V_{1}^{\prime}\right|-1+\left|V_{2}^{\prime}\right|-1-1=p-3$, a contradiction.

Claim 5.6. We have $\left|V_{i}\right|=\left|C\left(V_{i}\right)\right|+1$ and $\left|V_{3-i}\right|=\left|C\left(V_{3-i}\right)\right|+2$ for some $i \in[2]$.

Proof. Recall that $\left|V_{i}\right| \geq\left|C\left(V_{i}\right)\right|+1$ for each $i \in$ [2] by Theorem 5.1. First suppose that $\left|V_{i}\right| \geq\left|C\left(V_{i}\right)\right|+2$ for all $i \in$ [2]. Note that for each $i \in[2]$, 
since $\left|V_{i}\right| \geq 2$, we have $\left|C\left(V_{i}\right)\right| \geq 1$ and thus $\left|V_{i}\right| \geq 3$. Moreover, if $\left|C\left(V_{1}\right)\right|=1$ (resp., $\left|C\left(V_{2}\right)\right|=1$ ), then $G\left[V_{1}\right]$ (resp., $G\left[V_{2}\right]$ ) is a monochromatic complete subgraph of order at least 3 , and if $\left|C\left(V_{1}\right)\right| \geq 2$ (resp., $\left|C\left(V_{2}\right)\right| \geq 2$ ), then $G\left[V_{1}\right]$ (resp., $G\left[V_{2}\right]$ ) contains a $\left(p^{\prime}-2\right)$-colored $K_{p^{\prime}}$ for every $4 \leq p^{\prime} \leq\left|V_{1}\right|$ (resp., $4 \leq p^{\prime} \leq\left|V_{2}\right|$ ) by Theorem 5.7. Thus we can choose a $\left(p_{i}-2\right)$-colored $K_{p_{i}}$ in $G\left[V_{i}\right]$ for each $i \in[2]$ such that $3 \leq p_{i} \leq\left|V_{i}\right|$ and $p_{1}+p_{2}=p$. So there is a $(p-3)$-colored $K_{p}$ in $G$, a contradiction. Hence, we may assume that $\left|V_{1}\right|=\left|C\left(V_{1}\right)\right|+1$ without loss of generality.

If $\left|V_{2}\right|=\left|C\left(V_{2}\right)\right|+1$, then $k+3=\left|V_{1}\right|+\left|V_{2}\right|=\left|C\left(V_{1}\right)\right|+\left|C\left(V_{2}\right)\right|+2$, so $\left|C\left(V_{1}\right)\right|+\left|C\left(V_{2}\right)\right|=k+1$. Then $C\left(V_{1}\right) \cap C\left(V_{2}\right) \neq \emptyset$. Let $C^{\prime}=C\left(V_{1}\right) \cap C\left(V_{2}\right)$, and we have $1 \notin C^{\prime}$ by Claim 5.5. If $1 \notin C\left(V_{1}\right)$ and $1 \notin C\left(V_{2}\right)$, then $\left|C^{\prime}\right| \geq 2$ (otherwise we have $\left|C\left(V_{1}\right)\right|+\left|C\left(V_{2}\right)\right| \leq k$ ). Then we can choose $V_{1}^{\prime} \subseteq V_{1}$ and $V_{2}^{\prime} \subseteq V_{2}$ with $\left|V_{1}^{\prime} \cup V_{2}^{\prime}\right|=p$ such that $\left|C\left(V_{1}^{\prime}\right) \cap C\left(V_{2}^{\prime}\right)\right| \geq 2$. Then $\left|C\left(V_{1}^{\prime} \cup V_{2}^{\prime}\right)\right| \leq$ $1+\left|C\left(V_{1}^{\prime}\right)\right|+\left|C\left(V_{2}^{\prime}\right)\right|-2 \leq 1+\left|V_{1}^{\prime}\right|-1+\left|V_{2}^{\prime}\right|-1-2=p-3$, a contradiction. Hence, without loss of generality, we may assume that $1 \in C\left(V_{1}\right), c_{0} \in C^{\prime}$ and $c_{0} \neq 1$. Then we can choose $V_{1}^{\prime} \subseteq V_{1}$ and $V_{2}^{\prime} \subseteq V_{2}$ with $\left|V_{1}^{\prime} \cup V_{2}^{\prime}\right|=p$ such that $\left\{1, c_{0}\right\} \subseteq C\left(V_{1}^{\prime}\right)$ and $c_{0} \in C\left(V_{2}^{\prime}\right)$. Then $\left|C\left(V_{1}^{\prime} \cup V_{2}^{\prime}\right)\right| \leq\left|C\left(V_{1}^{\prime}\right)\right|+\left|C\left(V_{2}^{\prime}\right)\right|-$ $1 \leq\left|V_{1}^{\prime}\right|-1+\left|V_{2}^{\prime}\right|-1-1=p-3$, a contradiction. Therefore, we have $\left|V_{2}\right| \geq\left|C\left(V_{2}\right)\right|+2$.

If $1 \leq\left|C\left(V_{2}\right)\right| \leq p-4$, then $\left|V_{2}\right| \leq p-2$ in order to avoid a $(p-3)$-colored $K_{p}$. Let $V_{1}^{\prime} \subseteq V_{1}$ such that $\left|V_{1}^{\prime} \cup V_{2}\right|=p$. Then $\left|V_{2}\right|=p-\left|V_{1}^{\prime}\right| \leq p-\left(\left|C\left(V_{1}^{\prime}\right)\right|+\right.$ $1) \leq p-\left(\left|C\left(V_{1}^{\prime} \cup V_{2}\right)\right|-\left|C\left(V_{2}\right)\right|\right) \leq p-\left(p-2-\left|C\left(V_{2}\right)\right|\right)=\left|C\left(V_{2}\right)\right|+2$, where the second inequality is by $\left|C\left(V_{1}^{\prime} \cup V_{2}\right)\right| \leq 1+\left|C\left(V_{1}^{\prime}\right)\right|+\left|C\left(V_{2}\right)\right|$, and the last inequality follows from the assumption that $G$ contains no $(p-3)$-colored $K_{p}$. If $p-3 \leq\left|C\left(V_{2}\right)\right| \leq k-1$, then by the induction hypothesis we have $\left|V_{2}\right| \leq\left|C\left(V_{2}\right)\right|+2$. If $\left|C\left(V_{2}\right)\right|=k$, then $\left|V_{2}\right|=k+3-\left|V_{1}\right| \leq k+2=\left|C\left(V_{2}\right)\right|+2$. Therefore, we have $\left|V_{2}\right|=\left|C\left(V_{2}\right)\right|+2$.

By Claim 5.6, we may assume that $\left|V_{1}\right|=\left|C\left(V_{1}\right)\right|+1$ and $\left|V_{2}\right|=\left|C\left(V_{2}\right)\right|+2$ without loss of generality.

Claim 5.7. $C\left(V_{1}\right) \cap C\left(V_{2}\right)=\emptyset$. 
Proof. For a contradiction, suppose that $C^{\prime}=C\left(V_{1}\right) \cap C\left(V_{2}\right) \neq \emptyset$. Similar to the second paragraph in the proof of Claim 5.6, we have $1 \notin C\left(V_{1}\right), 1 \notin C\left(V_{2}\right)$ and $\left|C^{\prime}\right|=1$, say $C^{\prime}=\left\{c_{0}\right\}$.

If $\left|V_{2}\right| \leq p-2$, then we can choose $V_{1}^{\prime} \subseteq V_{1}$ such that $\left|V_{1}^{\prime} \cup V_{2}\right|=p$ and $c_{0} \in C\left(V_{1}^{\prime}\right)$. Now we have $\left|C\left(V_{1}^{\prime} \cup V_{2}\right)\right| \leq 1+\left|C\left(V_{1}^{\prime}\right)\right|+\left|C\left(V_{2}\right)\right|-1 \leq$ $1+\left(\left|V_{1}^{\prime}\right|-1\right)+\left(\left|V_{2}\right|-2\right)-1=p-3$, a contradiction. Thus $\left|V_{2}\right| \geq p-1$ and $\left|C\left(V_{2}\right)\right|=\left|V_{2}\right|-2 \geq p-3$. Let $u v$ be an edge within $V_{1}$ with $c(u v)=c_{0}$. Then $G\left[V_{2} \cup\{u, v\}\right]$ is a $\left(\left|C\left(V_{2}\right)\right|+1\right)$-colored $K_{\left|V_{2}\right|+2}$. If $\left|C\left(V_{1}\right)\right| \geq 2$, then $\left|C\left(V_{2} \cup\{u, v\}\right)\right| \leq k-1$, and thus we can derive a contradiction by the induction hypothesis. Thus we have $C\left(V_{1}\right)=\left\{c_{0}\right\}$ and $\left|V_{1}\right|=2$.

By Theorem 5.7, we may assume that $H$ is a copy of a $(p-5)$-colored $K_{p-3}$ in $G\left[V_{2}\right]$. If $c_{0} \in C(H)$, then $G\left[V(H) \cup V_{1}\right]$ is a $(p-4)$-colored $K_{p-1}$. For any vertex $w \in V_{2} \backslash V(H)$, we have $\left|C\left(w, V(H) \cup V_{1}\right) \backslash C\left(V(H) \cup V_{1}\right)\right| \leq 1$ by Lemma 5.14, which implies a $(p-3)$-colored $K_{p}$, a contradiction. If $c_{0} \notin C(H)$ and there is an edge $x y$ with color $c_{0}$ such that $x \in V(H)$ and $y \in V_{2} \backslash V(H)$, then $C(y, V(H)) \backslash C(H)=\left\{c_{0}\right\}$ by Lemma 5.14. Then $G\left[V(H) \cup V_{1} \cup\{y\}\right]$ is a $(p-3)$-colored $K_{p}$, a contradiction. Hence, $G\left[V_{2}\right]$ contains no edge in color $c_{0}$ which has an end-vertex in $V(H)$. Thus we may assume that $x y$ is an edge with color $c_{0}$ such that $x, y \in V_{2} \backslash V(H)$. By Theorem 5.7, we may further assume that $H^{\prime}$ is a copy of a $(p-6)$-colored $K_{p-4}$ in $H$. By Lemma 5.14, we have $\left|C\left(x, V\left(H^{\prime}\right)\right) \backslash C\left(H^{\prime}\right)\right| \leq 1$ and $C\left(y, V\left(H^{\prime}\right) \cup\{x\}\right) \backslash C\left(V\left(H^{\prime}\right) \cup\{x\}\right)=\left\{c_{0}\right\}$. Then $G\left[V\left(H^{\prime}\right) \cup V_{1} \cup\{x, y\}\right]$ is a $(p-3)$-colored $K_{p}$, a contradiction.

By Claim 5.6, we have $\left|C\left(V_{1}\right)\right|+\left|C\left(V_{2}\right)\right|=\left|V_{1}\right|+\left|V_{2}\right|-3=k$. Then we have either $1 \in C\left(V_{1}\right)$ or $1 \in C\left(V_{2}\right)$ by Claims 5.5 and 5.7. We first consider the case $1 \in C\left(V_{1}\right)$ and $1 \notin C\left(V_{2}\right)$. We define a subset $V_{2}^{\prime} \subseteq V_{2}$ as follows. If $\left|V_{2}\right| \leq p-3$, then $V_{2}^{\prime}=V_{2}$. If $\left|V_{2}\right| \geq p-2$, then we choose $V_{2}^{\prime}$ such that $G\left[V_{2}^{\prime}\right]$ is a ( $\left.p-4\right)$-colored $K_{p-2}$ (using Theorem 5.7). Then let $V_{1}^{\prime} \subset V_{1}$ such that $\left|V_{1}^{\prime}\right|=p-\left|V_{2}^{\prime}\right|$ and $1 \in C\left(V_{1}^{\prime}\right)$. Since $\left|C\left(V_{1}^{\prime} \cup V_{2}^{\prime}\right)\right| \leq\left|C\left(V_{1}^{\prime}\right)\right|+\left|C\left(V_{2}^{\prime}\right)\right| \leq$ $\left|V_{1}^{\prime}\right|-1+\left|V_{2}^{\prime}\right|-2=p-3$, we derive a contradiction. Next, we consider the case $1 \notin C\left(V_{1}\right)$ and $1 \in C\left(V_{2}\right)$. In this case, we have $\left|V_{2}\right| \geq p$, since otherwise if $\left|V_{2}\right| \leq p-1$, then we can choose $V_{1}^{\prime} \subset V_{1}$ with $\left|V_{1}^{\prime} \cup V_{2}\right|=p$ such that $\left|C\left(V_{1}^{\prime} \cup V_{2}\right)\right| \leq\left|C\left(V_{1}^{\prime}\right)\right|+\left|C\left(V_{2}\right)\right| \leq\left|V_{1}^{\prime}\right|-1+\left|V_{2}\right|-2=p-3$, a contradiction. 
By Theorem 5.7, we may assume that $H$ is a copy of a $(p-4)$-colored $K_{p-2}$ in $G\left[V_{2}\right]$. Let $u$ be any vertex in $V_{1}$. If $1 \in C(H)$, then $G[V(H) \cup\{u\}]$ is a $(p-4)$-colored $K_{p-1}$. For any vertex $v \in V_{2} \backslash V(H)$, we have $\mid C(v, V(H) \cup$ $\{u\}) \backslash C(V(H) \cup\{u\}) \mid \leq 1$ by Lemma 5.14, which implies a $(p-3)$-colored $K_{p}$, a contradiction. If $1 \notin C(H)$ and there is an edge $x y$ with color 1 such that $x \in V(H)$ and $y \in V_{2} \backslash V(H)$, then $C(y, V(H)) \backslash C(H)=\{1\}$ by Lemma 5.14. Then $G[V(H) \cup\{u, y\}]$ is a $(p-3)$-colored $K_{p}$, a contradiction. If $1 \notin C(H)$ and $G\left[V_{2}\right]$ contains no edge with color 1 incident with a vertex of $H$, then we may assume that $x y$ is an edge with color 1 such that $x, y \in V_{2} \backslash V(H)$. By Theorem 5.7, we may further assume that $H^{\prime}$ is a copy of a $(p-5)$ colored $K_{p-3}$ in $H$. By Lemma 5.14, we have $\left|C\left(x, V\left(H^{\prime}\right)\right) \backslash C\left(H^{\prime}\right)\right| \leq 1$ and $C\left(y, V\left(H^{\prime}\right) \cup\{x\}\right) \backslash C\left(V\left(H^{\prime}\right) \cup\{x\}\right)=\{1\}$. Then $G\left[V\left(H^{\prime}\right) \cup\{x, y, u\}\right]$ is a $(p-3)$-colored $K_{p}$. This contradiction completes the proof of Theorem 5.9.

Remark 5.1. The bound $p \geq 8$ in Theorem 5.9 is best possible. Indeed, if $p=7$, then we can show that $g_{4}^{5}(7)>8$ by the following example. Let $G_{1}$ (resp., $G_{2}$ ) be a $K_{4}$ using colors 1 and 2 (resp., colors 3 and 4 ) such that colors 1 and 2 (resp., colors 3 and 4) induce two monochromatic copies of a $P_{4}$. Let $G$ be a 5-colored $K_{8}$ obtained by joining $G_{1}$ and $G_{2}$ using edges that all get color 5. It is easy to check that $G$ contains neither a rainbow $K_{3}$ nor a 4-colored $K_{7}$. When $p=6$, we can prove that $g_{3}^{4}(6)=8$ and $g_{3}^{5}(6)=10$ (see Section 5.8). When $p=5$, the function $g_{2}^{k}(5)$ is exponential in $k$ by Theorem 5.10.

\subsection{Proofs of Theorems 5.10 and 5.11}

We first present our proof of Theorem 5.10.

Proof of Theorem 5.10. We first show that there is a Gallai-k-coloring of $K_{2^{k}}$, in which there is no $K_{5}$ receiving at most two distinct colors. For $k=2$, let $G_{2}$ be an edge-coloring of $K_{4}$ with colors 1 and 2 such that color 1 induces a perfect matching and color 2 induces a $C_{4}$. It is easy to check that there is neither a rainbow $K_{3}$ nor a monochromatic $K_{3}$ in $G_{2}$, and $G_{2}$ contains no 2-colored $K_{5}$ clearly. Suppose for some $2 \leq i \leq k-1$ we have constructed a 
Gallai-i-coloring $G_{i}$ of $K_{2^{i}}$ in which there is neither a monochromatic $K_{3}$ nor a 2-colored $K_{5}$. Then we construct an $(i+1)$-edge-coloring $G_{i+1}$ of $K_{2^{i+1}}$ by joining two copies of $G_{i}$ with edges that all get color $i+1$. Since $G_{i}$ contains no rainbow $K_{3}$, there is no rainbow $K_{3}$ in $G_{i+1}$. Since $G_{i}$ contains neither a monochromatic $K_{3}$ nor a 2-colored $K_{5}$, there is no 2-colored $K_{5}$ in $G_{i+1}$. By repeating this process, we finally obtain a Gallai-k-coloring $G_{k}$ of $K_{2^{k}}$ without a 2-colored $K_{5}$.

We now prove that $g_{2}^{k}(5) \leq 2^{k}+1$ by induction on $k$. For the base case, it is trivial that $g_{2}^{2}(5)=5$. Now assume that it holds for every $2 \leq k^{\prime} \leq k-1$, and we will prove it for $k \geq 3$.

For a contradiction, suppose that $G$ is a Gallai-k-coloring of $K_{2^{k}+1}$ without a 2-colored $K_{5}$. Using Theorem 1.4 , let $V_{1}, V_{2}, \ldots, V_{m}(m \geq 2)$ be a Gallai partition of $V(G)$. We choose it such that $m$ is minimum. Since there is no 2-colored $K_{5}$, we have $m \leq 4$. If $m=4$, then by the minimality of $m$, there are exactly two colors used between the parts, say colors 1 and 2 . In order to avoid a 2-colored $K_{5}$, there is no edge with color 1 or 2 within each part. If $k=3$, then there is only color 3 within these parts. Note that $\max _{1 \leq i<j \leq 4}\left|V_{i} \cup V_{j}\right| \geq 5$, so there is a 2-colored $K_{5}$. Thus $k \geq 4$. By the induction hypothesis, we have $|V(G)|=\left|V_{1}\right|+\left|V_{2}\right|+\left|V_{3}\right|+\left|V_{4}\right| \leq 4 \cdot 2^{k-2}=2^{k}$, a contradiction.

Thus $2 \leq m \leq 3$, and by the minimality of $m$ we may assume $m=2$ and $c\left(V_{1}, V_{2}\right)=1$. If $1 \notin C\left(V_{1}\right)$ and $1 \notin C\left(V_{2}\right)$, then by the induction hypothesis, we have $|V(G)|=\left|V_{1}\right|+\left|V_{2}\right| \leq 2^{k-1}+2^{k-1}=2^{k}$, a contradiction. If $1 \in C\left(V_{1}\right)$ and $1 \in C\left(V_{2}\right)$, then $G$ contains a monochromatic $K_{4}$. By Lemma 5.14, $G$ contains a 2-colored $K_{5}$, a contradiction. Thus we may assume that $1 \in C\left(V_{1}\right)$ and $1 \notin C\left(V_{2}\right)$ without loss of generality.

Claim 5.8. Color 1 induces a bipartite graph within $V_{1}$.

Proof. We first show that $G\left[V_{1}\right]$ contains no monochromatic $K_{3}$ with color 1 . Otherwise, suppose $\{u, v, w\}$ forms a monochromatic $K_{3}$ with color 1 within $V_{1}$. Then for any vertex $x \in V_{2}$, we have that $\{u, v, w, x\}$ forms a monochromatic $K_{4}$. By Lemma 5.14, there is a 2-colored $K_{5}$ in $G$, a contradiction.

We next show that $G\left[V_{1}\right]$ contains no $C_{4}$ with exactly three edges in color

1. Otherwise, if $G\left[V_{1}\right]$ contains such $C_{4}$, say $c(u v)=c(v w)=c(w z)=1$ 
and $c(z u)=2$. In order to avoid a rainbow $K_{3}$, we have $c(u w) \in\{1,2\}$ and $c(v z) \in\{1,2\}$. Then for any vertex $x \in V_{2}$, we have that $\{u, v, w, z, x\}$ forms a 2-colored $K_{5}$, a contradiction.

Finally, we show that $G\left[V_{1}\right]$ contains no monochromatic odd cycle in color 1 (thus color 1 induces a bipartite graph within $V_{1}$ ). Suppose that $C_{2 t+1}=$ $a_{1} a_{2} \cdots a_{2 t+1} a_{1}(t \geq 2)$ is a monochromatic cycle using color 1 in $G\left[V_{1}\right]$. Since there is no $C_{4}$ with exactly three edges in color 1 , we have $c\left(a_{1} a_{4}\right)=1$, so $c\left(a_{1} a_{6}\right)=1, c\left(a_{1} a_{8}\right)=1, \cdots, c\left(a_{1} a_{2 t}\right)=1$. Then $\left\{a_{1}, a_{2 t}, a_{2 t+1}\right\}$ forms a monochromatic $K_{3}$ in color 1 , a contradiction.

Let $E_{1}$ be the set of edges with color 1 in $G\left[V_{1}\right]$, and let $V_{1}^{\prime} \subseteq V_{1}$ be the set of vertices incident with some edge of $E_{1}$. By Claim 5.8, we may partition $V_{1}^{\prime}$ into two parts $A$ and $B$ such that $1 \notin C(A)$ and $1 \notin C(B)$. Since $1 \in C\left(V_{1}\right)$, we have $A \neq \emptyset$ and $B \neq \emptyset$. Let $V_{1}^{\prime \prime}=V_{1} \backslash V_{1}^{\prime}$ (it is possible that $V_{1}^{\prime \prime}=\emptyset$ ).

Claim 5.9. The following statements hold:

(1) for any color $i \in C\left(V_{1}^{\prime}\right)$, we have $i \notin C\left(V_{2}\right)$;

(2) $\left|V_{2}\right| \leq 2^{\left|C\left(V_{2}\right)\right|}$;

(3) $|A| \leq 2^{|C(A)|}$ and $|B| \leq 2^{|C(B)|}$.

Proof. (1) If $i=1$, then it holds clearly. If $i \neq 1$, then we may assume that $c(u v)=i$ for some $u, v \in V_{1}^{\prime}$. Since $u \in V_{1}^{\prime}$, there exists a vertex $w \in V_{1}^{\prime} \backslash\{u, v\}$ with $c(u w)=1$. In order to avoid a rainbow $K_{3}$, we have $c(v w) \in\{1, i\}$. If $i \in$ $C\left(V_{2}\right)$, then there is a 2-colored $K_{5}$ using colors 1 and $i$ in $G$, a contradiction.

(2) Let $\left|C\left(V_{2}\right)\right|=j(0 \leq j \leq k-1)$. If $j=0$, then $\left|V_{2}\right|=1=2^{0}$. If $j=1$, then $G\left[V_{2}\right]$ is a monochromatic complete subgraph. If $\left|V_{2}\right| \geq 3$, then $G$ contains a 2-colored $K_{5}$ since $1 \in C\left(V_{1}\right)$, a contradiction. Thus $\left|V_{2}\right|=2=2^{1}$. If $2 \leq j \leq k-1$, then $\left|V_{2}\right| \leq 2^{j}$ by the induction hypothesis.

(3) By symmetry, we only prove it for $A$. If $|C(A)| \neq 1$, then $|A| \leq 2^{|C(A)|}$ by the same argument as in (2). If $|C(A)|=1$, then $G[A]$ is a monochromatic complete subgraph. Suppose $|A| \geq 3$, say $u, v, w \in A$. Recall that $1 \notin C(A)$. By the definition of $A$ and $B$, there exists a vertex $x \in B$ such that $c(u x)=1$. In order to avoid a rainbow $K_{3}$, we have $C(x,\{v, w\}) \subseteq C(A) \cup\{1\}$. Note that for 
any vertex $y \in V_{2}$, we have $c(y,\{u, v, w, x\})=1$. Thus $\{u, v, w, x, y\}$ forms a 2-colored $K_{5}$, a contradiction.

Claim 5.10. $|A| \geq 2$ and $|B| \geq 2$.

Proof. By symmetry, we only prove $|A| \geq 2$. If $|A|=1$, say $A=\{u\}$, then we have $c(u, B)=1$ by the definition of $B$. By Claim 5.9 (1), we have $C(B) \cap C\left(V_{2}\right)=\emptyset$, so $|C(B)|+\left|C\left(V_{2}\right)\right| \leq k-1$. If $V_{1}^{\prime \prime}=\emptyset$, then by Claim 5.9 (2) and (3) we have $|V(G)|=|A|+|B|+\left|V_{2}\right| \leq 1+2^{|C(B)|}+2^{\left|C\left(V_{2}\right)\right|} \leq 1+$ $\left(2^{|C(B)|+\left|C\left(V_{2}\right)\right|}+1\right) \leq 1+2^{k-1}+1<2^{k}+1$, a contradiction. Thus $V_{1}^{\prime \prime} \neq \emptyset$, say $v \in V_{1}^{\prime \prime}$. Note that $v$ is not incident with any edge in color 1 . Thus we may further assume that $c(u v)=2$. In order to avoid a rainbow $K_{3}$, we have $c(v, B)=2$. Then $2 \notin C(B)$ and $2 \notin C\left(V_{2}\right)$ in order to avoid a 2-colored $K_{5}$, so $|C(B)|+\left|C\left(V_{2}\right)\right| \leq k-2$. Then $|V(G)|=|A|+|B|+\left|V_{2}\right|+\left|V_{1}^{\prime \prime}\right| \leq 1+2^{|C(B)|}+$ $2^{\left|C\left(V_{2}\right)\right|}+2^{k-1} \leq 1+\left(2^{|C(B)|+\left|C\left(V_{2}\right)\right|}+1\right)+2^{k-1} \leq 1+2^{k-2}+1+2^{k-1}<2^{k}+1$, a contradiction.

Claim 5.11. $C(B) \backslash C(A) \neq \emptyset$ and $C(A) \backslash C(B) \neq \emptyset$.

Proof. By symmetry, we only prove $C(B) \backslash C(A) \neq \emptyset$. Recall that $1 \notin C(A)$ and $1 \notin C(B)$. By Claim 5.10, we have $|C(B)| \geq 1$. Since $G[B]$ is a Gallai-coloring, there exists a color, say color 2 , inducing a connected spanning subgraph of $G[B]$ by Corollary 5.13. We will show that $2 \notin C(A)$. For a contradiction, suppose that there are two vertices $u, v \in A$ with $c(u v)=2$. We may further assume that $c(u w)=1$ and $c(w x)=2$, where $w, x \in B$. Then $c(v w) \in\{1,2\}$ and $c(u x) \in\{1,2\}$. Thus $c(v x) \notin\{1,2\}$, since otherwise $\{u, v, w, x\}$ together with a vertex in $V_{2}$ forms a 2-colored $K_{5}$. Then $c(v w)=c(u x)=2$ in order to avoid a rainbow $K_{3}$. Since $v$ is incident with some edge in color 1, we may assume that $c(v y)=1$ for some $y \in B \backslash\{w, x\}$. In order to avoid a rainbow $K_{3}$, we have $C(y,\{u, w\}) \subseteq\{1,2\}$. Then $\{u, v, w, y\}$ together with a vertex in $V_{2}$ forms a 2-colored $K_{5}$, a contradiction.

By Claim 5.9 (1), we have $C(A) \cap C\left(V_{2}\right)=\emptyset$ and $C(B) \cap C\left(V_{2}\right)=\emptyset$. Recall that $1 \notin C(A)$ and $1 \notin C\left(B \cup V_{1}^{\prime \prime}\right)$. By Claim 5.11, we further have $|C(A)| \leq k-|\{1\}|-|C(B) \backslash C(A)|-\left|C\left(V_{2}\right)\right| \leq k-2-\left|C\left(V_{2}\right)\right|$. Thus $|V(G)|=$ 
$|A|+\left|V_{2}\right|+\left|B \cup V_{1}^{\prime \prime}\right| \leq 2^{k-2-\left|C\left(V_{2}\right)\right|}+2^{\left|C\left(V_{2}\right)\right|}+2^{k-1} \leq 2^{k-2}+1+2^{k-1}<2^{k}+1$, a contradiction. This completes the proof of Theorem 5.10.

Next, we present our proof of Theorem 5.11.

Proof of Theorem 5.11. By Theorem 5.10, we have $g_{2}^{q}(5)=2^{q}+1 \leq p$. Thus every Gallai- $q$-colored $K_{p}$ contains a 2-colored $K_{5}$. Let $g=g_{q}^{k}(p)$. Then every Gallai- $k$-coloring of $K_{g}$ contains a Gallai- $q$-colored $K_{p}$, and thus a 2-colored $K_{5}$. Hence, $g_{q}^{k}(p) \geq g_{2}^{k}(5)=2^{k}+1$.

In fact, we can generalize Theorem 5.11 as follows.

Theorem 5.20. For integers $p, q, k$ with $q \leq \log _{2}(p-1)$ and $k \geq q$, we have $g_{q}^{k}(p) \geq\left\lfloor(p-1)^{1 / q}\right\rfloor^{k}+1$.

Proof. Let $a:=\left\lfloor(p-1)^{1 / q}\right\rfloor$. We show that there is a Gallai- $k$-coloring of $K_{a^{k}}$, in which there is no $K_{p}$ receiving at most $q$ distinct colors. Let $G_{1}$ be a monochromatic copy of $K_{a}$ with color 1 . Suppose for some $1 \leq i \leq k-1$ we have constructed a Gallai-i-coloring $G_{i}$ of $K_{a^{i}}$. Then we construct an $(i+1)$ edge-coloring $G_{i+1}$ of $K_{a^{i+1}}$ by joining a copies of $G_{i}$ using edges that all get color $i+1$. Finally, we obtain a Gallai- $k$-coloring $G_{k}$ of $K_{a^{k}}$. For any $q$ distinct colors $c_{1}, c_{2}, \ldots, c_{q}$, the largest complete subgraph in $G_{k}$ using only these $q$ colors has order at most $a^{q} \leq p-1$. Thus $g_{q}^{k}(p) \geq a^{k}+1$.

\subsection{Proof of Theorem $\mathbf{5 . 1 2}$}

We first introduce an additional definition and prove some useful lemmas. An exact Gallai-k-coloring is a Gallai- $k$-coloring in which all the $k$ colors are used.

Lemma 5.21. For any $n \geq 2$, there are at least $\lceil n / 2\rceil$ colors each inducing $a$ star in every exact Gallai- $(n-1)$-coloring of $K_{n}$.

Proof. We prove the statement by induction on $n$. For the base case, if $2 \leq n \leq 3$, the statement clearly holds. Now assume that it holds for every $2 \leq n^{\prime} \leq n-1$, and we will prove it for $n$. Let $G$ be an exact Gallai- $(n-1)$ coloring of $K_{n}$. Let $V_{1}, V_{2}, \ldots, V_{m}$ be a Gallai partition of $V(G)$ such that $m$ is 
minimum. If $m \geq 4$, then by Theorem 5.1 we have $|C(G)| \leq 2+\sum_{i=1}^{m}\left|C\left(V_{i}\right)\right| \leq$ $2+\sum_{i=1}^{m}\left(\left|V_{i}\right|-1\right) \leq 2+n-m \leq n-2$, a contradiction. Thus $2 \leq m \leq 3$, so $m=2$ by the minimality of $m$. Without loss of generality, we may assume that $c\left(V_{1}, V_{2}\right)=1$ and $\left|V_{1}\right| \geq\left|V_{2}\right|$. We claim that $1 \notin C\left(V_{1}\right) \cup C\left(V_{2}\right)$ and $C\left(V_{1}\right) \cap C\left(V_{2}\right)=\emptyset$, since otherwise $|C(G)| \leq 1+\left|C\left(V_{1}\right)\right|+\left|C\left(V_{2}\right)\right|-1 \leq$ $\left|V_{1}\right|-1+\left|V_{2}\right|-1 \leq n-2$. If $\left|V_{2}\right|=1$, then $G\left[V_{1}\right]$ is an exact Gallai- $(n-2)$ coloring of $K_{n-1}$. By the induction hypothesis, the number of colors each inducing a star is at least $1+\lceil(n-1) / 2\rceil \geq\lceil n / 2\rceil$. If $\left|V_{1}\right| \geq\left|V_{2}\right| \geq 2$, then by the induction hypothesis, the number of colors each inducing a star is at least $\left\lceil\left|V_{1}\right| / 2\right\rceil+\left\lceil\left|V_{2}\right| / 2\right\rceil \geq\lceil n / 2\rceil$.

Lemma 5.22. For integers $c, n, N$ with $c \geq 2, n \geq 2(7+c)^{c}$ and $N \geq n-\frac{3 n}{(7+c)^{c}}$, we have $\frac{N}{(2+c)(6+c)^{c-1}}-2 \geq \frac{n}{(7+c)^{c}}$.

Proof. Let $a=6+c$. Then $a \geq 8$. Since

$$
\begin{aligned}
& (2+c)(6+c)^{c-1}\left(\frac{N}{(2+c)(6+c)^{c-1}}-2-\frac{n}{(7+c)^{c}}\right) \\
\geq & \left(n-\frac{3 n}{(7+c)^{c}}\right)-2(2+c)(6+c)^{c-1}-\frac{(2+c)(6+c)^{c-1} n}{(7+c)^{c}} \\
= & \left(1-\frac{3}{(7+c)^{c}}-\frac{(2+c)(6+c)^{c-1}}{(7+c)^{c}}\right) n-2(2+c)(6+c)^{c-1} \\
\geq & \left(1-\frac{3}{(7+c)^{c}}-\frac{(2+c)(6+c)^{c-1}}{(7+c)^{c}}\right) 2(7+c)^{c}-2(2+c)(6+c)^{c-1} \\
= & 2(7+c)^{c}-4(2+c)(6+c)^{c-1}-6 \\
= & 2\left((a+1)^{a-6}-2(a-4) a^{a-7}\right)-6 \\
= & 2\left(\sum_{i=0}^{a-6}\left(\begin{array}{c}
a-6 \\
i
\end{array}\right) a^{i}-2 a^{a-6}+8 a^{a-7}\right)-6 \\
= & 2\left(\sum_{i=0}^{a-8}\left(\begin{array}{c}
a-6 \\
i
\end{array}\right) a^{i}+(a-6) a^{a-7}+a^{a-6}-2 a^{a-6}+8 a^{a-7}\right)-6 \\
= & 2 \sum_{i=0}^{a-8}\left(\begin{array}{c}
a-6 \\
i
\end{array}\right) a^{i}+4 a^{a-7}-6 \geq 0,
\end{aligned}
$$


we have $\frac{N}{(2+c)(6+c)^{c-1}}-2 \geq \frac{n}{(7+c)^{c}}$.

Lemma 5.23. For any $c \geq 1$ and $n \geq 2(7+c)^{c}$, there are at least $\left\lceil n /(7+c)^{c}\right\rceil$ colors each inducing a star in every exact Gallai- $(n-c)$-coloring of $K_{n}$.

Proof. We prove the statement by induction on $c$. For the base case, if $c=1$, the statement holds by Lemma 5.21. Now assume that it holds for every $1 \leq c^{\prime} \leq c-1$, and we will prove it for $c$ with $c \geq 2$. Let $G$ be an exact Gallai- $(n-c)$-coloring of $K_{n}$ using colors $1,2, \ldots, n-c$. For a contradiction, suppose that the number of colors each inducing a star in $G$ is less than $\frac{n}{(7+c)^{c}}$.

Claim 5.12. Let $N$ be an integer satisfying $N \leq n$ and $\frac{N}{(2+c)(6+c)^{c-1}}-2 \geq \frac{n}{(7+c)^{c}}$. For any $V^{\prime} \subseteq V(G)$ with $\left|V^{\prime}\right|=N, C\left(V^{\prime}\right) \cap C\left(V^{\prime}, V(G) \backslash V^{\prime}\right)=\emptyset$ and $C\left(V^{\prime}\right) \cap$ $C\left(V(G) \backslash V^{\prime}\right)=\emptyset$, let $G^{\prime}=G\left[V^{\prime}\right]$. If $G^{\prime}$ is an exact Gallai- $(N-c)$-coloring of $K_{N}$, then $V\left(G^{\prime}\right)$ has a Gallai partition consisting of exactly two parts $V_{1}^{\prime}$ and $V_{2}^{\prime}$, such that $\left|C\left(V_{1}^{\prime}\right)\right|=\left|V_{1}^{\prime}\right|-c,\left|C\left(V_{2}^{\prime}\right)\right|=\left|V_{2}^{\prime}\right|-1, c\left(V_{1}^{\prime}, V_{2}^{\prime}\right) \notin C\left(V_{1}^{\prime}\right) \cup C\left(V_{2}^{\prime}\right)$ and $C\left(V_{1}^{\prime}\right) \cap C\left(V_{2}^{\prime}\right)=\emptyset$.

Proof. Note that the integer $N$ and subset $V^{\prime}$ satisfying the above conditions exist since we can choose $N=n, V^{\prime}=V(G)$ and $G^{\prime}=G$. Without loss of generality, let $C\left(G^{\prime}\right)=[N-c]$. Firstly, we assume that there exists some color $\ell \in[N-c]$ such that the subgraph of $G^{\prime}$ induced by color $\ell$ has at least two nontrivial components. Then we recolor all the edges of color $\ell$ in one of its nontrivial components with color $N-c+1$. Let $G^{\prime \prime}$ be the resulting coloring of $K_{N}$. It is easy to check that $G^{\prime \prime}$ is an exact Gallai- $(N-(c-1))$-coloring of $K_{N}$. Since $\frac{N}{(2+c)(6+c)^{c-1}}-2 \geq \frac{n}{(7+c)^{c}}$, we have $N \geq 2(7+(c-1))^{c-1}$. By the induction hypothesis, there are at least $\frac{N}{(7+(c-1))^{c-1}}$ colors each inducing a star in $G^{\prime \prime}$. Recall that $C\left(V^{\prime}\right) \cap C\left(V^{\prime}, V(G) \backslash V^{\prime}\right)=\emptyset$ and $C\left(V^{\prime}\right) \cap C\left(V(G) \backslash V^{\prime}\right)=\emptyset$. There are at least $\frac{N}{(7+(c-1))^{c-1}}-2 \geq \frac{n}{(7+c)^{c}}$ colors each inducing a star in $G$, a contradiction.

Next, we may assume that every color induces a subgraph with exactly one nontrivial component in $G^{\prime}$. Let $V_{1}^{\prime}, V_{2}^{\prime}, \ldots, V_{m}^{\prime}$ be a Gallai partition of $V\left(G^{\prime}\right)$ such that $m$ is minimum and $\left|V_{1}^{\prime}\right|=\max _{1 \leq i \leq m}\left\{\left|V_{i}^{\prime}\right|\right\}$, and let $S$ be the set of colors used between these parts. Then $1 \leq|S| \leq 2$ and for every $1 \leq i<j \leq m$, we have $\left(C\left(V_{i}^{\prime}\right) \cap C\left(V_{j}^{\prime}\right)\right) \backslash S=\emptyset$. 
If $m \geq 4$, then $N-c=\left|C\left(G^{\prime}\right)\right| \leq|S|+\sum_{i=1}^{m}\left|C\left(V_{i}^{\prime}\right)\right| \leq 2+\sum_{i=1}^{m}\left(\left|V_{i}^{\prime}\right|-1\right) \leq$ $N+2-m$, so $m \leq 2+c$. Thus $\left|V_{1}^{\prime}\right| \geq N /(2+c) \geq 2(6+c)^{c-1}=2(7+(c-1))^{c-1}$. Moreover, $\left|C\left(V_{1}^{\prime}\right)\right| \geq N-c-|S|-\sum_{i=2}^{m}\left|C\left(V_{i}^{\prime}\right)\right| \geq N-c-2-\sum_{i=2}^{m}\left(\left|V_{i}^{\prime}\right|-1\right)=$ $\left|V_{1}^{\prime}\right|-c+m-3 \geq\left|V_{1}^{\prime}\right|-c+1$. Let $C\left(V_{1}^{\prime}\right)=\left\{c_{i}: i=1,2, \ldots,\left|C\left(V_{1}^{\prime}\right)\right|\right\}$. For all $\left|V_{1}^{\prime}\right|-c+2 \leq j \leq\left|C\left(V_{1}^{\prime}\right)\right|$ (if $\left|C\left(V_{1}^{\prime}\right)\right|>\left|V_{1}^{\prime}\right|-c+1$ ), we recolor all the edges of color $c_{j}$ with color $c_{1}$ in $G^{\prime}\left[V_{1}^{\prime}\right]$, so we obtain an exact Gallai- $\left(\left|V_{1}^{\prime}\right|-(c-1)\right)$ coloring of $K_{\left|V_{1}^{\prime}\right|}$. By the induction hypothesis, there are at least $\frac{\left|V_{1}^{\prime}\right|}{(7+(c-1))^{c-1}}$ colors each inducing a star in $G^{\prime}\left[V_{1}^{\prime}\right]$. Thus the number of colors each inducing a star in $G$ is at least $\frac{\left|V_{1}^{\prime}\right|}{(7+(c-1))^{c-1}}-|S| \geq \frac{N}{(2+c)(7+(c-1))^{c-1}}-2 \geq \frac{n}{(7+c)^{c}}$, a contradiction.

Thus $2 \leq m \leq 3$, so $m=2$ by the minimality of $m$. Then $\left|V_{1}^{\prime}\right| \geq N / 2 \geq$ $2(7+(c-1))^{c-1}$. Note that $\left|C\left(V_{1}^{\prime}\right)\right| \geq N-c-\left|C\left(V_{2}^{\prime}\right)\right|-|S| \geq N-c-\left|V_{2}^{\prime}\right|+1-1=$ $\left|V_{1}^{\prime}\right|-c$. If $\left|C\left(V_{1}^{\prime}\right)\right| \geq\left|V_{1}^{\prime}\right|-c+1$, then we can derive a contradiction by a similar argument as above. Thus we have $\left|C\left(V_{1}^{\prime}\right)\right|=\left|V_{1}^{\prime}\right|-c$, so $\left|C\left(V_{2}^{\prime}\right)\right|=\left|V_{2}^{\prime}\right|-1$, $S \cap\left(C\left(V_{1}^{\prime}\right) \cup C\left(V_{2}^{\prime}\right)\right)=\emptyset$ and $C\left(V_{1}^{\prime}\right) \cap C\left(V_{2}^{\prime}\right)=\emptyset$.

We will use an algorithm to find $\left\lceil n /(7+c)^{c}\right\rceil$ colors each inducing a star in $G$. Let $V_{1}^{(0)}:=V(G), V_{2}^{(0)}:=\emptyset, G^{(0)}:=G, t:=1, A:=\emptyset$ and $B:=\emptyset$. The algorithm at time $i(i \geq 1)$ consists of two steps.

Step 1. By applying Claim 5.12 to $N=\left|V_{1}^{(i-1)}\right|, V^{\prime}=V_{1}^{(i-1)}$ and $G^{\prime}=$ $G\left[V_{1}^{(i-1)}\right]$, we obtain a Gallai partition $V_{1}^{(i)}, V_{2}^{(i)}$ of $V\left(G^{\prime}\right)$ such that $\left|C\left(V_{1}^{(i)}\right)\right|$ $=\left|V_{1}^{(i)}\right|-c,\left|C\left(V_{2}^{(i)}\right)\right|=\left|V_{2}^{(i)}\right|-1, c\left(V_{1}^{(i)}, V_{2}^{(i)}\right) \notin C\left(V_{1}^{(i)}\right) \cup C\left(V_{2}^{(i)}\right)$ and $C\left(V_{1}^{(i)}\right) \cap C\left(V_{2}^{(i)}\right)=\emptyset$.

Step 2. If $\left|V_{2}^{(i)}\right|=1$, then let $c_{t}=c\left(V_{1}^{(i)}, V_{2}^{(i)}\right), t:=t+1$ and $A:=A \cup V_{2}^{(i)}$; otherwise if $\left|V_{2}^{(i)}\right| \geq 2$, then let $B:=B \cup V_{2}^{(i)}$.

We repeat the above steps until $t \geq \frac{n}{(7+c)^{c}}+1$. Finally, we obtain $t-1$ distinct colors $c_{1}, c_{2}, \ldots, c_{t-1}$ each inducing a star in $G$. It remains to show that the above algorithm is valid. Since for any $j \leq i-1$ with $V_{2}^{(j)} \subseteq B$ we have $\left|V_{2}^{(j)}\right| \geq 2$ and $\left|C\left(V_{2}^{(j)}\right)\right|=\left|V_{2}^{(j)}\right|-1$, the number of colors each inducing a star in $G\left[V_{2}^{(j)}\right]$ is at least $\left[\left|V_{2}^{(j)}\right| / 2\right]$ by Lemma 5.21. Recall that $c\left(V_{1}^{(j)}, V_{2}^{(j)}\right) \notin C\left(V_{1}^{(j)}\right) \cup C\left(V_{2}^{(j)}\right)$ and $C\left(V_{1}^{(j)}\right) \cap C\left(V_{2}^{(j)}\right)=\emptyset$ for every $j \leq i-1$. Thus $|B|<\frac{2 n}{(7+c)^{c}}$; otherwise the number of colors each inducing a star in $G$ is at least $\frac{n}{(7+c)^{c}}$, a contradiction. Thus $\left|V_{1}^{(i-1)}\right|=n-|B|-|A|>n-\frac{3 n}{(7+c)^{c}}$. By 
Lemma 5.22, $\left|V_{1}^{(i-1)}\right|$ satisfies the condition of $N$ in Claim 5.12. Moreover, $V_{1}^{(i-1)}$ (resp., $G^{(i-1)}$ ) satisfies the condition of $V^{\prime}$ (resp., $G^{\prime}$ ) in Claim 5.12. Thus we can apply Claim 5.12 in Step 1, so the algorithm is valid.

Now we have all ingredients to present our proof of Theorem 5.12.

Proof of Theorem 5.12. The cases $c \in\{1,2\}$ follow from Theorems 5.7 and 5.9 , so we may assume that $c \geq 3$. The lower bound $g_{k-1}^{k}(p)>p$ is trivial. For the upper bound, let $G$ be a Gallai- $k$-coloring of $K_{p+1}$. We may assume that $G$ is an exact Gallai- $k$-coloring, where $k=p-c=p+1-(c+1)$. By Lemma 5.23, the number of colors each inducing a star in $G$ is at least $\frac{p+1}{(7+(c+1))^{c+1}} \geq 2$. Let $i$ be a color that induces a star in $G$, and let $v$ be a vertex with maximum degree in this star. Then $G-v$ is a copy of $K_{p}$ using at most $k-1$ colors. The result follows.

\subsection{Two results for $K_{6}$}

In this section, we shall prove $g_{3}^{4}(6)=8$ and $g_{3}^{5}(6)=10$.

Theorem 5.24. $g_{3}^{4}(6)=8$.

Proof. We first show that $g_{3}^{4}(6)>7$ by construction. Taking a copy of $K_{7}$ with vertex set $U \cup\{x, y, z\}$, where $U=\{u, v, w, s\}$, we color the edges such that $c(u v)=c(v w)=c(w s)=1, c(v s)=c(s u)=c(u w)=2, c(x, U)=c(z, U)=$ $c(z y)=3$ and $c(y, U)=c(y x)=c(x z)=4$. It is easy to check that the resulting coloring is a Gallai-4-coloring of $K_{7}$ without a 3-colored $K_{6}$.

Next we prove that $g_{3}^{4}(6) \leq 8$. For a contradiction, suppose that $G$ is a Gallai-4-colored $K_{8}$ containing no 3-colored $K_{6}$ and $V(G)=\left\{u_{1}, u_{2}, \ldots, u_{8}\right\}$.

Claim 5.13. There is no vertex $u \in V(G)$ such that $|C(u, V(G-u))|=1$.

Proof. Suppose there exists a vertex $u \in V(G)$ with $|C(u, V(G-u))|=1$, say $c\left(u_{8}, V\left(G-u_{8}\right)\right)=1$. Let $F$ be the spanning subgraph of $G-u_{8}$ consisting of all the edges in color 1 . Let $S \subseteq V\left(G-u_{8}\right)$ be a minimum covering of $F$ and $T=V(F) \backslash S=V(G) \backslash\left(S \cup\left\{u_{8}\right\}\right)$. In order to avoid a 3-colored $K_{6}$, we have $|S| \geq 2$. 
We next show that $F$ is a bipartite graph. Firstly, $F$ contains no $C_{3}$; otherwise $G$ contains a monochromatic $K_{4}$ in color 1 , so there is a 3-colored $K_{6}$ by Lemma 5.14. Secondly, suppose that $F$ contains a copy $W$ of $C_{5}$. It is easy to check that $|C(V(W)) \cap\{2,3,4\}| \leq 2$ in order to avoid a rainbow triangle, so $W \cup\left\{u_{8}\right\}$ forms a 3-colored $K_{6}$, a contradiction. Thus $F$ contains no $C_{5}$. Finally, suppose that $F$ contains a copy of $C_{7}$, say $u_{1} u_{2} \cdots u_{7} u_{1}$. Since $F$ contains no $C_{3}$, we may assume that $c\left(u_{1} u_{3}\right)=2$ without loss of generality. In order to avoid a rainbow triangle in $G$ or a $C_{5}$ in $F$, we have $c\left(u_{1} u_{4}\right)=2$. Then $c\left(u_{2} u_{4}\right)=2$ in order to avoid a rainbow triangle in $G$ or a $C_{3}$ in $F$. Now $\left\{u_{8}, u_{1}, u_{2}, u_{3}, u_{4}\right\}$ forms a 2-colored $K_{5}$. By Lemma 5.14, there is a 3-colored $K_{6}$ in $G$. This contradiction implies that $F$ contains no odd cycle and thus $F$ is a bipartite graph.

Since $F$ is bipartite, we have $|S| \leq\left\lfloor\left|V\left(G-u_{8}\right)\right| / 2\right\rfloor=3$. If $|S|=3$, then $|T|=4$, say $S=\left\{u_{1}, u_{2}, u_{3}\right\}$ and $T=\left\{u_{4}, u_{5}, u_{6}, u_{7}\right\}$. By Theorem 5.15, there is a matching of size 3 in $F$, i.e., $G-u_{8}$ contains three pairwise nonadjacent edges in color 1 . It is easy to check that these three edges must appear between $S$ and $T$. Without loss of generality, we may assume that $c\left(u_{1} u_{4}\right)=$ $c\left(u_{2} u_{5}\right)=c\left(u_{3} u_{6}\right)=1$. Note that $1 \notin C(T)$, so we may further assume that $C\left(\left\{u_{4}, u_{5}, u_{6}\right\}\right) \subseteq\{2,3\}$. Then $C\left(S,\left\{u_{4}, u_{5}, u_{6}\right\}\right) \subseteq\{1,2,3\}$ in order to avoid a rainbow triangle. Now $S$ forms a monochromatic $K_{3}$ in color 4; otherwise there is a 3-colored $K_{6}$ within $S \cup\left\{u_{8}, u_{4}, u_{5}, u_{6}\right\}$. Since $G$ is a Gallai-coloring, it is easy to see that $c\left(S,\left\{u_{4}, u_{5}, u_{6}\right\}\right)=1$. Then $S \cup\left\{u_{8}, u_{4}, u_{5}\right\}$ forms a 3-colored $K_{6}$, a contradiction. Therefore, we have $|S|=2$ and $|T|=5$, say $S=\left\{u_{1}, u_{2}\right\}$ and $T=\left\{u_{3}, u_{4}, \ldots, u_{7}\right\}$. Note that $C(T) \subseteq\{2,3,4\}$, so there is a 2-colored $K_{4}$ within $T$ by Theorem 5.7. Without loss of generality, we may assume that $C(X) \subseteq\{2,3\}$, where $X=\left\{u_{3}, u_{4}, u_{5}, u_{6}\right\}$. Moreover, we may assume that $c\left(u_{1} u_{3}\right)=1$ by Theorem 5.15. Then $C\left(X \cup\left\{u_{1}\right\}\right)=\{1,2,3\}$ by Lemma 5.14, which implies that $X \cup\left\{u_{8}, u_{1}\right\}$ forms a 3-colored $K_{6}$, a contradiction.

Let $V_{1}, V_{2}, \ldots, V_{m}$ be a Gallai partition of $V(G)$ such that $m$ is minimum. If there is only one color between these parts, then we assume that color 1 is this color, and if there are two colors between these parts, then we assume that colors 1 and 2 are these two colors.

Claim 5.14. $m \leq 3$. 
Proof. Suppose $m \geq 4$. In order to avoid a 3-colored $K_{6}$, the following statements hold: (1) $m=4$; (2) $1,2 \notin \bigcup_{i=1}^{m} C\left(V_{i}\right)$; (3) color 3 (resp., color 4) is used in exactly one of these parts; (4) there is neither a 2-colored $K_{5}$ nor a monochromatic $K_{3}$ within each part. Thus we have $\sum_{i=1}^{m}\left|V_{i}\right| \leq$ $\max \{4+1+1+1,2+2+1+1\}=7<|V(G)|$, a contradiction.

By Claim 5.14 and the minimality of $m$, we have $m=2$. Note that at most one of $1 \in C\left(V_{1}\right)$ and $1 \in C\left(V_{2}\right)$ holds; otherwise $G$ contains a monochromatic $K_{4}$ and thus $G$ contains a 3-colored $K_{6}$ by Lemma 5.14. Firstly, we consider the case that $1 \notin C\left(V_{1}\right)$ and $1 \notin C\left(V_{2}\right)$, i.e., $C\left(V_{i}\right) \subseteq\{2,3,4\}$ for $i \in[2]$. In this case, we have $\left|V_{1}\right| \leq 5$ and $\left|V_{2}\right| \leq 5$, so $\left|V_{1}\right| \geq 3$ and $\left|V_{2}\right| \geq 3$. We claim that there is no 2-colored $K_{4}$ within each $V_{i}$ for $i \in$ [2]. Indeed, if there is a 2-colored copy $K$ of $K_{4}$ in some $V_{i}$, say $i=1$ and $C(K) \subseteq\{2,3\}$, then $C\left(V_{2}\right)=\{4\}$ in order to avoid a 3-colored $K_{6}$. If $\left|V_{2}\right|=4$, then it is easy to find a 3-colored $K_{6}$ in $G$. If $\left|V_{2}\right|=3$, then $\left|V_{1}\right|=5$. Now $G$ also contains a 3 -colored $K_{6}$ no matter whether $4 \in C\left(V_{1}\right)$ or $4 \notin C\left(V_{1}\right)$. Hence, there is no 2-colored $K_{4}$ within each $V_{i}$ for $i \in[2]$. By Theorem 5.7, we have $g_{2}^{3}(4)=5$, so $\left|V_{i}\right| \leq 4$ for $i \in[2]$, which implies that $\left|V_{1}\right|=\left|V_{2}\right|=4$ and moreover, $C\left(V_{1}\right)=C\left(V_{2}\right)=\{2,3,4\}$. For any two distinct colors $c_{1}, c_{2} \in\{2,3,4\}$, we say that $c_{1}$ and $c_{2}$ are adjacent if there are two adjacent edges $e$ and $f$ such that $c(e)=c_{1}$ and $c(f)=c_{2}$. Note that if $c_{1}$ and $c_{2}$ are adjacent, then there is a 2-colored $K_{3}$ with colors $c_{1}$ and $c_{2}$. Without loss of generality, we may assume that colors 2 and 3 are adjacent in $G\left[V_{1}\right]$. Then colors 2 and 3 are nonadjacent in $G\left[V_{2}\right]$, and thus color 4 is adjacent to both color 2 and color 3 in $G\left[V_{2}\right]$. But then color 4 is adjacent to neither color 2 nor color 3 in $G\left[V_{1}\right]$, a contradiction.

Finally, we consider the case that exactly one of $1 \in C\left(V_{1}\right)$ and $1 \in C\left(V_{2}\right)$ holds, say $1 \in C\left(V_{1}\right)$ and $1 \notin C\left(V_{2}\right)$.

Claim 5.15. The following statements hold:

(1) there is no monochromatic $K_{3}$ in color 1 within $V_{1}$;

(2) $C\left(V_{1}\right) \cap C\left(V_{2}\right)=\emptyset$. 
Proof. If (1) does not hold, then $G$ contains a monochromatic $K_{4}$. Thus there is a 3-colored $K_{6}$ by Lemma 5.14. This contradiction proves (1). We next prove (2). Suppose that $C\left(V_{1}\right) \cap C\left(V_{2}\right) \neq \emptyset$, say $2 \in C\left(V_{1}\right) \cap C\left(V_{2}\right)$. If colors 1 and 2 are adjacent in $G\left[V_{1}\right]$, then $G$ contains a 2-colored $K_{5}$. By Lemma 5.14, there is a 3-colored $K_{6}$, a contradiction. Thus colors 1 and 2 are nonadjacent in $G\left[V_{1}\right]$, and we may assume that $u_{1}, u_{2}, u_{3}, u_{4} \in V_{1}$ such that $c\left(u_{1} u_{2}\right)=1$, $c\left(u_{3} u_{4}\right)=2$ and $1,2 \notin C\left(\left\{u_{1}, u_{2}\right\},\left\{u_{3}, u_{4}\right\}\right)$. Since $G$ is a Gallai-coloring, we have $\left|C\left(\left\{u_{1}, u_{2}\right\},\left\{u_{3}, u_{4}\right\}\right)\right|=1$. Assume that $u_{5} u_{6}$ is an edge with color 2 in $G\left[V_{2}\right]$. Then $\left\{u_{1}, u_{2}, \ldots, u_{6}\right\}$ forms a 3 -colored $K_{6}$, a contradiction. This completes the proof of (2).

In order to avoid a 3-colored $K_{6}$, we have $\left|V_{2}\right| \leq 5$, so $\left|V_{1}\right| \geq 3$. Then $\left|C\left(V_{1}\right)\right| \geq 2$ by Claim 5.15 (1). If $\left|C\left(V_{1}\right)\right|=2$, then $\left|C\left(V_{2}\right)\right|=2$ by Claim 5.15 (2). In this case, we have $\left|V_{1}\right| \leq 3$ (resp., $\left|V_{2}\right| \leq 4$ ) in order to avoid a 3colored $K_{6}$. Then $\left|V_{1}\right|+\left|V_{2}\right| \leq 3+4<|V(G)|$, a contradiction. If $\left|C\left(V_{1}\right)\right|=3$, then $\left|C\left(V_{2}\right)\right|=1$ by Claim 5.15 (2). In this case, we have $\left|V_{1}\right| \leq 4$ (resp., $\left.\left|V_{2}\right| \leq 3\right)$ in order to avoid a 3-colored $K_{6}$. Then $\left|V_{1}\right|+\left|V_{2}\right| \leq 4+3<|V(G)|$, a contradiction. If $\left|C\left(V_{1}\right)\right|=4$, then $\left|C\left(V_{2}\right)\right|=0$ by Claim 5.15 (2). In this case, we have $\left|V_{2}\right|=1$, contradicting to Claim 5.13. This contradiction completes the proof.

Theorem 5.25. $g_{3}^{5}(6)=10$.

Proof. We first show that $g_{3}^{5}(6)>9$ by construction. Taking a copy of $K_{9}$ with vertex set $U \cup V \cup\{x, y\}$, where $U=\{r, s, t\}$ and $V=\{u, v, w, z\}$, we color the edges such that $c(\{x, y\}, U \cup V)=1, c(x y)=c(\{u, z\},\{v, w\})=2$, $c(r s)=c(s t)=c(v w)=3, c(r t)=c(u z)=4$ and $c(U, V)=5$. Let $G^{\prime}$ be the resulting coloring. It is easy to check that $G^{\prime}$ is a Gallai-coloring. Moreover, for any two distinct colors $i, j \in[5]$, we need to delete at least four vertices such that there is neither color $i$ nor color $j$ on edges of the remaining graph. Thus $G^{\prime}$ is a Gallai-5-coloring of $K_{9}$ without a 3-colored $K_{6}$.

Next we prove that $g_{3}^{5}(6) \leq 10$. For a contradiction, suppose that $G$ is a Gallai-5-colored $K_{10}$ containing no 3-colored $K_{6}$ and $V(G)=\left\{u_{1}, u_{2}, \ldots, u_{10}\right\}$. Let $V_{1}, V_{2}, \ldots, V_{m}$ be a Gallai partition of $V(G)$ such that $m$ is minimum. If there is only one color between these parts, then we assume that color 1 is 
this color, and if there are two colors between these parts, then we assume that colors 1 and 2 are these two colors.

If $m \geq 4$, then in order to avoid a 3 -colored $K_{6}$, the following statements hold: (1) $m=4$; (2) $1,2 \notin \bigcup_{i=1}^{m} C\left(V_{i}\right)$; (3) for each $i \in\{3,4,5\}$, color $i$ is used in exactly one of these parts; (4) there is neither a 2-colored $K_{5}$ nor a monochromatic $K_{3}$ within each part. Thus $\sum_{i=1}^{m}\left|V_{i}\right| \leq \max \{5+3 \cdot 1,4+2+$ $2 \cdot 1,2+2+2+1\}=8<|V(G)|$, a contradiction. Hence, $2 \leq m \leq 3$. By the minimality of $m$, we have $m=2$. Note that at most one of $1 \in C\left(V_{1}\right)$ and $1 \in C\left(V_{2}\right)$ holds; otherwise $G$ contains a monochromatic $K_{4}$ and thus $G$ contains a 3-colored $K_{6}$ by Lemma 5.14 . We divide the rest of the proof into two cases.

Case 1. $1 \notin C\left(V_{1}\right)$ and $1 \notin C\left(V_{2}\right)$.

In this case, we have $C\left(V_{i}\right) \subseteq\{2,3,4,5\}$ for $i \in[2]$. Since $g_{3}^{4}(6)=8$, we have $\left|V_{i}\right| \leq 7$ and thus $\left|V_{3-i}\right| \geq 3$ for each $i \in[2]$. We claim that there is no 2-colored $K_{4}$ within each $V_{i}$ for $i \in$ [2]. Indeed, if there is a 2-colored copy $K$ of $K_{4}$ in some $V_{i}$, say $i=1$ and $C(K) \subseteq\{2,3\}$, then $C\left(V_{2}\right) \subseteq\{4,5\}$ in order to avoid a 3-colored $K_{6}$. Thus $\left|V_{2}\right| \leq 4$ for avoiding a 3-colored $K_{6}$. If $\left|V_{2}\right|=4$, then $4,5 \notin C\left(V_{1}\right)$, which implies that $G\left[V_{1}\right]$ is a 2-colored $K_{6}$, a contradiction. If $\left|V_{2}\right|=3$, then $\left|V_{1}\right|=7$. In order to avoid a 3-colored $K_{6}$, we have $C\left(V_{1}\right)=\{2,3,4,5\}$, colors 4 and 5 are nonadjacent in $G\left[V_{1}\right]$, and $G\left[V_{1}\right]$ contains no monochromatic $K_{3}$ in color 4 or 5 . If $G\left[V_{1}\right]$ contains a monochromatic $2 K_{2}$ in color 4 and a monochromatic $2 K_{2}$ in color 5 , then $\left|V_{1}\right| \geq 8$, a contradiction. Thus we may assume that $G\left[V_{1}\right]$ contains no monochromatic $2 K_{2}$ in color 4 . Then there is a vertex $u \in V_{1}$ such that $G\left[V_{1} \backslash\{u\}\right]$ contains no edge in color 4, which implies that $G\left[V_{1} \backslash\{u\}\right]$ is a 3 -colored $K_{6}$, a contradiction. Hence, there is no 2-colored $K_{4}$ within each $V_{i}$ for $i \in[2]$.

By Theorem 5.7, we have $g_{2}^{4}(4)=6$, so $\left|V_{i}\right| \leq 5$ for $i \in[2]$, which implies that $\left|V_{1}\right|=\left|V_{2}\right|=5$ and moreover, $C\left(V_{1}\right)=C\left(V_{2}\right)=\{2,3,4,5\}$ since $g_{2}^{3}(4)=5$. Without loss of generality, we may assume that colors 2 and 3 are adjacent in $G\left[V_{1}\right]$. Recall that if two distinct colors $c_{1}$ and $c_{2}$ are adjacent, then there is a 2-colored $K_{3}$ with colors $c_{1}$ and $c_{2}$. Then colors 2 and 3 are nonadjacent in $G\left[V_{2}\right]$. We may assume that $u_{1}, u_{2}, u_{3}, u_{4} \in V_{2}$ such that $c\left(u_{1} u_{2}\right)=2$ and 
$c\left(u_{3} u_{4}\right)=3$. Then $C\left(\left\{u_{1}, u_{2}\right\},\left\{u_{3}, u_{4}\right\}\right) \subseteq\{4,5\}$. In order to avoid a rainbow triangle, there is exactly one color on the edges between $\left\{u_{1}, u_{2}\right\}$ and $\left\{u_{3}, u_{4}\right\}$, say color 4 . Then color 4 is adjacent to neither color 2 nor color 3 in $G\left[V_{1}\right]$, so color 4 is adjacent to color 5 in $G\left[V_{1}\right]$. Since $\left|V_{2}\right|=5$ and $G\left[V_{2}\right]$ contains a monochromatic $K_{2,2}$ in color 4 , we have that colors 4 and 5 are adjacent in $G\left[V_{2}\right]$. Then $G$ contains a 3-colored $K_{6}$ with colors 1,4 and 5, a contradiction.

Case 2. Exactly one of $1 \in C\left(V_{1}\right)$ and $1 \in C\left(V_{2}\right)$ holds.

Without loss of generality, we may assume $1 \in C\left(V_{1}\right)$ and $1 \notin C\left(V_{2}\right)$. We have $C\left(V_{1}\right) \cap C\left(V_{2}\right)=\emptyset$ by the same argument as in the proof of Claim 5.15. Thus $\left|C\left(V_{1}\right)\right|+\left|C\left(V_{2}\right)\right|=5$. For avoiding a 3-colored $K_{6}$ and since $g_{3}^{4}(6)=8$, we have the following inequality:

$$
\left|V_{1}\right|+\left|V_{2}\right| \leq \begin{cases}2+7, & \text { if }\left|C\left(V_{1}\right)\right|=1 \text { and }\left|C\left(V_{2}\right)\right|=4 \\ 3+5, & \text { if }\left|C\left(V_{1}\right)\right|=2 \text { and }\left|C\left(V_{2}\right)\right|=3 \\ 4+4, & \text { if }\left|C\left(V_{1}\right)\right|=3 \text { and }\left|C\left(V_{2}\right)\right|=2 \\ 6+2, & \text { if }\left|C\left(V_{1}\right)\right|=4 \text { and }\left|C\left(V_{2}\right)\right|=1\end{cases}
$$

contradicting the fact that $|V(G)|=10$. Thus it suffices to consider the case $\left|C\left(V_{1}\right)\right|=5$ and $\left|C\left(V_{2}\right)\right|=0$. In this case, we have $\left|V_{2}\right|=1$, say $V_{2}=\left\{u_{10}\right\}$. Let $F$ be the spanning subgraph of $G\left[V_{1}\right]$ consisting of all the edges in color 1. Let $S \subseteq V_{1}$ be a minimum covering of $F$ and $T=V_{1} \backslash S$. Note that $C(T) \subseteq\{2,3,4,5\}$. Since $g_{3}^{4}(6)=8$, we have $|T| \leq 7$ and thus $|S| \geq 2$.

We next show that $F$ is a bipartite graph. By similar arguments as in the proof of Claim 5.13, there is no $C_{\ell}$ in $F$ for $\ell \in\{3,5,7\}$. We now show that $F$ contains no $C_{9}$. Suppose for a contradiction that $u_{1} u_{2} \cdots u_{9} u_{1}$ is a cycle in $F$. Since $F$ contains no $C_{3}$, we may assume that $c\left(u_{1} u_{3}\right)=2$ without loss of generality. In order to avoid a rainbow triangle in $G$ or a $C_{7}$ in $F$, we have $c\left(u_{1} u_{4}\right)=2$. Then $c\left(u_{2} u_{4}\right)=2$ in order to avoid a rainbow triangle in $G$ or a $C_{3}$ in $F$. Now $\left\{u_{10}, u_{1}, u_{2}, u_{3}, u_{4}\right\}$ forms a 2-colored $K_{5}$. By Lemma 5.14, there is a 3-colored $K_{6}$ in $G$. This contradiction implies that $F$ contains no odd cycle and thus $F$ is a bipartite graph.

Since $F$ is bipartite, we have $|S| \leq\left\lfloor\left|V_{1}\right| / 2\right\rfloor=4$. 
Claim 5.16. $|S|=2$ and $|T|=7$.

Proof. If $|S|=3$ and $|T|=6$ (resp., $|S|=4$ and $|T|=5$ ), then $F$ contains a matching of size 3 (resp., 4) by Theorem 5.15. It is easy to check that this matching must appear between $S$ and $T$. Without loss of generality, let $u_{1}, u_{2}, u_{3} \in S, u_{4}, u_{5}, \ldots, u_{8} \in T$, and $c\left(u_{1} u_{4}\right)=c\left(u_{2} u_{5}\right)=c\left(u_{3} u_{6}\right)=$ 1. Recall that $1 \notin C(T)$, so we may further assume that $C\left(\left\{u_{4}, u_{5}, u_{6}\right\}\right) \subseteq$ $\{2,3\}$. Then $C\left(\left\{u_{1}, u_{2}, u_{3}\right\},\left\{u_{4}, u_{5}, u_{6}\right\}\right) \subseteq\{1,2,3\}$ in order to avoid a rainbow triangle. Now $1,2,3 \notin C\left(\left\{u_{1}, u_{2}, u_{3}\right\}\right)$; otherwise there is a 3-colored $K_{6}$ in $G\left[\left\{u_{1}, u_{2}, \ldots, u_{6}, u_{10}\right\}\right]$. Thus $C\left(\left\{u_{1}, u_{2}, u_{3}\right\}\right) \subseteq\{4,5\}$. Then $c\left(\left\{u_{1}, u_{2}, u_{3}\right\}\right.$, $\left.\left\{u_{4}, u_{5}, u_{6}\right\}\right)=1$ in order to avoid a rainbow triangle. Note that $1 \notin C(T)$. If $C\left(u_{7},\left\{u_{4}, u_{5}, u_{6}\right\}\right) \cap\{4,5\} \neq \emptyset$, say $c\left(u_{7} u_{4}\right)=4$, then $C\left(u_{7},\left\{u_{1}, u_{2}, u_{3}\right\}\right) \subseteq$ $\{1,4\}$, which implies that $\left\{u_{1}, u_{2}, u_{3}, u_{4}, u_{7}, u_{10}\right\}$ forms a 3-colored $K_{6}$, a contradiction. Thus $C\left(u_{7},\left\{u_{4}, u_{5}, u_{6}\right\}\right) \subseteq\{2,3\}$. Then $c\left(u_{1} u_{7}\right) \in\{1,2,3\}$, which implies that $\left\{u_{1}, u_{4}, u_{5}, u_{6}, u_{7}, u_{10}\right\}$ forms a 3 -colored $K_{6}$, a contradiction.

By Claim 5.16 and Theorem 5.15, we may assume that $S=\left\{u_{1}, u_{2}\right\}, T=$ $\left\{u_{3}, u_{4}, \ldots, u_{9}\right\}$ and $c\left(u_{1} u_{3}\right)=c\left(u_{2} u_{4}\right)=1$ without loss of generality. Since $1 \notin C(T)$, we may further assume $c\left(u_{3} u_{4}\right)=2$. Then $c\left(u_{1} u_{4}\right), c\left(u_{2} u_{3}\right) \in\{1,2\}$, so $c\left(u_{1} u_{2}\right) \notin\{1,2\}$; otherwise $\left\{u_{1}, u_{2}, u_{3}, u_{4}, u_{10}\right\}$ forms a 2-colored $K_{5}$ and thus $G$ contains a 3-colored $K_{6}$ by Lemma 5.14. Without loss of generality, let $c\left(u_{1} u_{2}\right)=3$. Then $c\left(u_{1} u_{4}\right)=c\left(u_{2} u_{3}\right)=1$ in order to avoid a rainbow triangle.

Claim 5.17. For any vertex $u \in T \backslash\left\{u_{3}, u_{4}\right\}$, we have $4 \in C\left(u,\left\{u_{3}, u_{4}\right\}\right) \subseteq$ $\{2,4\}$ or $5 \in C\left(u,\left\{u_{3}, u_{4}\right\}\right) \subseteq\{2,5\}$.

Proof. Firstly, we have $3 \notin C\left(u,\left\{u_{3}, u_{4}\right\}\right)$; otherwise it is easy to check that $C\left(\left\{u, u_{1}, u_{2}, u_{3}, u_{4}, u_{10}\right\}\right)=\{1,2,3\}$ in order to avoid a rainbow triangle, which is a contradiction. Secondly, we have $C\left(u,\left\{u_{3}, u_{4}\right\}\right) \cap\{4,5\} \neq \emptyset$; otherwise if $c\left(u u_{3}\right)=c\left(u u_{4}\right)=2$, then $C\left(\left\{u, u_{1}, u_{2}, u_{3}, u_{4}, u_{10}\right\}\right)=\{1,2,3\}$. This contradiction completes the proof of Claim 5.17.

By Claim 5.17 and the Pigeonhole Principle, we may assume that $4 \in$ $C\left(u_{i},\left\{u_{3}, u_{4}\right\}\right) \subseteq\{2,4\}$ for $i \in\{5,6,7\}$. 
Claim 5.18. For each $i \in\{5,6,7\}$, we have $c\left(u_{i},\left\{u_{3}, u_{4}\right\}\right)=4$.

Proof. For a contradiction, suppose that $C\left(u_{i},\left\{u_{3}, u_{4}\right\}\right)=\{2,4\}$ for some $i \in\{5,6,7\}$, say $c\left(u_{5} u_{3}\right)=2$ and $c\left(u_{5} u_{4}\right)=4$. Then $c\left(\left\{u_{1}, u_{2}\right\}, u_{5}\right)=$ 1. If $c\left(u_{6} u_{3}\right)=4$, then $C\left(u_{6},\left\{u_{1}, u_{4}, u_{5}\right\}\right) \subseteq\{1,2,4\}$, which implies that $G\left[\left\{u_{1}, u_{3}, u_{4}, u_{5}, u_{6}, u_{10}\right\}\right]$ is a 3-colored $K_{6}$, a contradiction. Thus $c\left(u_{6} u_{3}\right)=2$, so $c\left(u_{6} u_{4}\right)=4$ since $4 \in C\left(u_{6},\left\{u_{3}, u_{4}\right\}\right)$. By symmetry, we have $c\left(u_{7} u_{3}\right)=2$ and $c\left(u_{7} u_{4}\right)=4$. Then $c\left(\left\{u_{1}, u_{2}\right\},\left\{u_{6}, u_{7}\right\}\right)=1$. If $C\left(\left\{u_{5}, u_{6}, u_{7}\right\}\right) \cap\{2,4\} \neq \emptyset$, then $G\left[\left\{u_{10}, u_{1}, u_{3}, u_{4}, \ldots, u_{7}\right\}\right]$ contains a 3 -colored $K_{6}$, a contradiction. Thus $C\left(\left\{u_{5}, u_{6}, u_{7}\right\}\right) \subseteq\{3,5\}$. Then $G\left[\left\{u_{10}, u_{1}, u_{2}, u_{5}, u_{6}, u_{7}\right\}\right]$ is a 3-colored $K_{6}$, a contradiction.

By Claim 5.18, we have $c\left(\left\{u_{5}, u_{6}, u_{7}\right\},\left\{u_{3}, u_{4}\right\}\right)=4$. In order to avoid a rainbow triangle, we have $C\left(\left\{u_{1}, u_{2}\right\},\left\{u_{5}, u_{6}, u_{7}\right\}\right) \subseteq\{1,4\}$. If $3 \in C\left(\left\{u_{5}, u_{6}\right.\right.$, $\left.u_{7}\right\}$ ) (resp., $\left.C\left(\left\{u_{5}, u_{6}, u_{7}\right\}\right) \cap\{2,4\} \neq \emptyset\right)$, then $G\left[\left\{u_{10}, u_{1}, u_{2}, u_{4}, u_{5}, u_{6}, u_{7}\right\}\right]$ (resp., $G\left[\left\{u_{10}, u_{1}, u_{3}, u_{4}, \ldots, u_{7}\right\}\right]$ ) contains a 3-colored $K_{6}$, a contradiction. Thus $c\left(\left\{u_{5}, u_{6}, u_{7}\right\}\right)=5$. Then $G\left[\left\{u_{10}, u_{1}, u_{4}, u_{5}, u_{6}, u_{7}\right\}\right]$ is a 3 -colored $K_{6}$. This contradiction completes the proof.

\subsection{Concluding remarks}

In this chapter, we studied the behavior of $g(n, p, q)$, which is the minimum number of colors that are needed for $K_{n}$ to have a Gallai-coloring in which every $K_{p}$ receives at least $q$ distinct colors. We now give a comparison of our results on $g(n, p, q)$ with the classical Erdős-Gyárfás function $f(n, p, q)$.

Firstly, Corollary 5.2 implies that $g(n, p, q)$ makes sense only for $2 \leq q \leq$ $p-1$, while $f(n, p, q)$ makes sense for $2 \leq q \leq\left(\begin{array}{l}p \\ 2\end{array}\right)$. Secondly, since we have an additional constraint on rainbow triangles, the value of $g(n, p, q)$ is at least that of $f(n, p, q)$ for $2 \leq q \leq p-1$. In fact, we have $g(n, p, q) \gg f(n, p, q)$ for certain values of $n, p$ and $k$. For instance, for appropriate $p$ and $n$, Theorems 5.7 and 5.9 imply that $g(n, p, p-1)=n-1$ and $g(n, p, p-2)=n-2$, while Conlon et al. [48] proved that $f(n, p, p-1)$ is subpolynomial in $n$. Thirdly, for appropriate $p$ and $n$, we have $g\left(n, p,\left\lceil\log _{2} p\right\rceil\right) \leq\left\lceil\log _{2} n\right\rceil$ (implied by Theorem 5.11) and $f\left(n, p,\left\lceil\log _{2} p\right\rceil\right) \leq\left\lceil\log _{2} n\right\rceil$ (see [62]). Moreover, we 
have $g(n, p, 2)=\Omega\left(\log _{2} n\right)$ by Theorem 5.6. Thus $g(n, p, q)=\Theta\left(\log _{2} n\right)$ for $2 \leq q \leq\left\lceil\log _{2} p\right\rceil$. But the best known upper and lower bounds on $r_{k}(p)$ (see Section 1.2) imply that $\frac{\log _{2} n}{p \log _{2} \log _{2} n} \leq f(n, p, 2) \leq \frac{8}{3}\left(\frac{1}{4}+\frac{1}{p-o(p)} \log _{2} n\right)$. Hence, it is possible that $f(n, p, q)=o\left(\log _{2} n\right)$ for some $q$ with $2 \leq q \leq\left\lceil\log _{2} p\right\rceil$. If this is true, then $f(n, p, q)=o(g(n, p, q))$ for these values of $q$. There is not much known about the classical Erdős-Gyárfás function $f(n, p, q)$ when $2 \leq q \leq p-2$. Further exploration of the behavior of $f(n, p, q)$ for $2 \leq q \leq p-2$ will help us to understand how the additional constraint on rainbow triangles forces the total number of colors to increase. Moreover, investigating the bounds on the multicolor Ramsey number $r_{k}(p)$ will also help to study the range of the values of $f(n, p, q)$.

Next, we present several open problems. Theorems 5.8 and 5.11 imply that $g\left(n, p,\left\lfloor(p-1)^{1 / 2}\right\rfloor\right)$ and $g\left(n, p,\left\lceil\log _{2} p\right\rceil\right)$ are at most $O\left(n^{1 / 2}\right)$ and $O(\log n)$, respectively. We know that $g(n, p, p-1)$ is linear in $n, g(n, p, 2)$ is logarithmic in $n$, and $g(n, p, q) \geq g(n, p, q-1)$. Thus for any fixed $p$ ( $p$ is large enough), there exists a value $q$ such that $g(n, p, q)$ is polynomial in $n$ and $g(n, p, q-1)$ is subpolynomial in $n$. A natural problem is to find the smallest $q$ such that $g(n, p, q)$ is polynomial in $n$, and the largest $q$ such that $g(n, p, q)$ is logarithmic in $n$.

Another natural problem is to find the threshold for linear $g(n, p, q)$, i.e., the smallest $q$ such that $g(n, p, q)$ is linear in $n$. We were not able to solve this problem, but in light of Theorems 5.7, 5.9 and 5.12, we pose the following problem.

Problem 5.1. Given a constant $c \geq 2$, does there exist a $p_{0}$ such that for all integers $p \geq p_{0}$ and $k \geq p-c$, we have $g_{p-c}^{k}(p)=k+c$ ? (Equivalently, given a constant $c^{\prime} \geq 1$, does there exist a $p_{0}$ such that for all integers $p \geq p_{0}$ and $n \geq p$, we have $g\left(n, p, p-c^{\prime}\right)=n-c^{\prime}$ ?)

The following construction shows that $g_{p-c}^{k}(p) \geq k+c$. Let $G$ be a copy of $K_{k+c-1}$ with vertex set $\left\{v_{1}, v_{2}, \ldots, v_{k+c-1}\right\}$. For every $1 \leq i \leq k$ and $i<j \leq k+c-1$, we color the edge $v_{i} v_{j}$ using color $i$, and we color all the remaining edges with color $k$. In the case $c \in\{2,3\}$, Theorems 5.7 and 5.9 answer Problem 5.1 positively. For $c \geq 4$, Theorem 5.12 shows that $g_{p-c}^{k}(p)=k+c$ for $k=p-c+1$ and sufficiently large $p$. 



\section{Chapter 6}

\section{Forbidden rainbow subgraphs that force large \\ monochromatic or 2-colored $k$-connected subgraphs}

The results in this chapter are motivated by considering and generalizing the following implication of Gallai-Ramsey numbers. If the Gallai-Ramsey number $g r_{m}\left(K_{3}: H\right)=n$, then forbidding a rainbow $K_{3}$ in an $m$-colored $K_{n}$ implies the existence of a monochromatic copy of $H$ in this $K_{n}$. A natural question is which stronger forbidden rainbow subgraph conditions imply a large $k$-connected monochromatic subgraph in such a $K_{n}$.

Let $n, k, m$ be positive integers with $n \gg m \gg k$, and let $\mathscr{G}$ (resp., $\mathscr{H}$ ) be the set of connected (resp., disconnected) graphs $G$ forcing that there exists a $k$-connected monochromatic subgraph of order at least $n-f(G, k, m)$ in every rainbow $G$-free exact $m$-edge-coloring of $K_{n}$, where $f(G, k, m)$ does not depend on $n$. The set $\mathscr{G}$ was determined by Fujita and Magnant in [81]. In this chapter, we give a complete characterization of $\mathscr{H}$, and we show that $\mathscr{G} \cup \mathscr{H}$ consists of precisely $P_{6}, P_{3} \cup P_{4}, K_{2} \cup P_{5}, K_{2} \cup 2 P_{3}, 2 K_{2} \cup K_{3}, 2 K_{2} \cup P_{4}^{+}$, $3 K_{2} \cup K_{1,3}$ and their subgraphs. 
We also consider an analogous problem for complete bipartite graphs. Let $s, t, k, m$ be positive integers with $\min \{s, t\} \gg m \gg k$, and let $\mathscr{B}$ be the set of bipartite graphs $H$ forcing that there exists a $k$-connected monochromatic subgraph of order at least $s+t-f(H, k, m)$ in any rainbow $H$-free exact $m$-edge-coloring of $K_{s, t}$, where $f(H, k, m)$ does not depend on $s$ or $t$. We prove that the set $\mathscr{B}$ consists of precisely $2 P_{3}, 2 K_{2} \cup K_{1,3}$ and their subgraphs.

Finally, we study how to force large $k$-connected 2-colored subgraphs instead of monochromatic subgraphs. We show that for $1 \leq k \leq 3$ and sufficiently large $n$, every Gallai-3-coloring of $K_{n}$ contains a $k$-connected subgraph of order at least $n-\lfloor(k-1) / 2\rfloor$ using at most two colors. For any positive integer $t$, we also show that the above statement is false for $k=4 t$.

\subsection{Introduction}

In 1983, Matula [140] studied the following Ramsey-type problem involving graph connectivity. Let $R(k ; m)$ be the smallest integer $n$ such that every $m$-edge-coloring of $K_{n}$ contains a $k$-connected monochromatic subgraph. For $m, k \geq 2$, Matula [140] proved that $2 m(k-1)+1 \leq R(k ; m)<10 m(k-1) / 3+1$ and $4 k-3 \leq R(k ; 2)<\left(3+(11 / 3)^{1 / 2}\right)(k-1)+1$. However, this result has no restriction on the order of the $k$-connected monochromatic subgraph.

It is easy to check that any 2-edge-coloring of $K_{n}$ contains a monochromatic spanning tree, hence a (1-)connected monochromatic subgraph of order $n$. In general, it is impossible to force a spanning monochromatic subgraph with a higher connectivity in every 2-colored $K_{n}$. For instance, consider a 2-edgecoloring of $K_{n}$ using red and blue such that the monochromatic subgraph induced by the red edges is a spanning star (only having connectivity 1 ). Then the spanning monochromatic subgraph with all the blue edges is $K_{1} \cup K_{n-1}$ and hence disconnected. Thus in order to force a monochromatic subgraph with a higher connectivity, we have to restrict ourselves to subgraphs with order smaller than $n$. In 2008, Bollobás and Gyárfás [21] posed the following conjecture involving forcing a monochromatic subgraph of order at least $n-2(k-1)$. 
Conjecture 6.1 (Bollobás and Gyárfás [21]). If $n>4(k-1)$ ), then every 2-edge-coloring of $K_{n}$ contains a monochromatic $k$-connected subgraph of order at least $n-2(k-1)$.

In [79], Fujita and Magnant proved that Conjecture 6.1 holds in case $n>6.5(k-1)$.

Theorem 6.1 (Fujita and Magnant [79]). If $n>6.5(k-1)$, then every 2-edgecoloring of $K_{n}$ contains a monochromatic $k$-connected subgraph of order at least $n-2(k-1)$.

Recently, Lo, Wu and Xie [132] proved that Conjecture 6.1 is true for $n \geq 5 k-\min \{\sqrt{4 k-2}+3,0.5 k+4\}$, and there is a counterexample with $n=5 k-2\lceil\sqrt{2 k-1}\rceil-3$.

For at least three colors, we adopt the following definition of an almost spanning $k$-connected monochromatic subgraph.

Definition 6.1 (Fujita and Magnant [81]). Given positive integers $k$ and $m$, for sufficiently large $n$ compared to $k$ and $m$, an almost spanning $k$-connected monochromatic subgraph, denoted by ASMS $(k)$, is a $k$-connected monochromatic subgraph of order at least $n-f(k, m)$ in an edge-colored $K_{n}$ using exactly $m$ colors, where $f(k, m)$ does not depend on $n$.

In the context of studying edge-colorings of $K_{n}$ using $m$ colors, it was shown that the largest order of a highly-connected monochromatic subgraph would be $n /(m-1)$ (see $[124,125,127])$. Thus, in order to force an $\operatorname{ASMS}(k)$, we must consider additional restrictions on the edge-coloring of $K_{n}$, such as forbidden proper subgraphs [105] or forbidden rainbow subgraphs [81]. Since we are focussing on edge-colorings, throughout this chapter we will not consider forbidden rainbow subgraphs with isolated vertices. Moreover, we will not consider forbidden rainbow subgraphs of order less than 3 either, for the simple reason that every edge-coloring of $K_{n}(n \geq 2)$ contains a rainbow $K_{2}$.

Let $P_{4}^{+}$be the unique tree with degree sequence $1,1,1,2,3$; note that we used $F_{2}$ to denote this tree in Chapter 2 (see Figure 2.1). In [81], Fujita and Magnant proved the following result. 
Theorem 6.2 (Fujita and Magnant [81]). Let $n, k, m$ be positive integers with $n \gg m \gg k$ and let $\mathscr{G}$ be the set of connected graphs $G$ of order at least 3 without isolated vertices such that there is an ASMS $(k)$ in any rainbow $G$-free exact m-edge-coloring of $K_{n}$. Then $\mathscr{G}$ consists of precisely $K_{3}, P_{6}, P_{4}^{+}$and their connected subgraphs of order at least 3.

In this chapter, in order to establish the counterpart of the above result for disconnected forbidden subgraphs, we first consider the following question.

Question 6.1. For what disconnected graphs $H$ of order at least 3 without isolated vertices does the following statement hold? Let $n, k, m$ be positive integers with $n \gg m \gg k$ and $m \geq|E(H)|$. There is an ASMS(k) in every rainbow $H$-free exact $m$-edge-coloring of $K_{n}$.

Let $\mathscr{H}$ be the set of disconnected graphs $H$ satisfying the statement in Question 6.1. Given a graph $H$, the component number $c_{0}(H)$ is the number of components of $H$. For each integer $i \geq 2$, let $\mathscr{H}^{(i)}:=\left\{H \mid c_{0}(H)=i, H \in \mathscr{H}\right\}$ and define $\mathscr{H}^{(1)}:=\mathscr{G}$. Clearly, we have $\mathscr{H}=\bigcup_{i \geq 2} \mathscr{H}^{(i)}$. We shall prove the following result in Section 6.2.

Theorem 6.3. The set $\mathscr{H}$ consists of precisely $P_{3} \cup P_{4}, K_{2} \cup P_{5}, K_{2} \cup 2 P_{3}, 2 K_{2} \cup K_{3}$, $2 K_{2} \cup P_{4}^{+}, 3 K_{2} \cup K_{1,3}$ and their disconnected subgraphs of order at least 3 without isolated vertices.

By Theorems 6.2 and 6.3, we have that $\mathscr{G} \cup \mathscr{H}$ consists of precisely $P_{6}$, $P_{3} \cup P_{4}, K_{2} \cup P_{5}, K_{2} \cup 2 P_{3}, 2 K_{2} \cup K_{3}, 2 K_{2} \cup P_{4}^{+}, 3 K_{2} \cup K_{1,3}$ and their subgraphs of order at least 3 without isolated vertices.

For any graph $G \notin \mathscr{G} \cup \mathscr{H}$, the largest monochromatic $k$-connected subgraph in a rainbow $G$-free edge-coloring of $K_{n}$ will not be almost spanning, but how large should it be? We shall prove the following additional result for a class of graphs in Section 6.3.

Theorem 6.4. For any graph $H \in \mathscr{G} \cup \mathscr{H}$, if $n$ is sufficiently larger than $m$ and $k$, then any rainbow $\left(P_{3} \cup H\right)$-free exact $m$-edge-coloring of $K_{n}$ contains a $k$-connected monochromatic subgraph of order at least $\beta n$, where $\beta=\beta(H)$ is a constant, not depending on $n, m$ or $k$. 
Another natural question is to consider an analogous problem for complete bipartite graphs instead of complete graphs.

Question 6.2. For what bipartite graphs $H$ of order at least 3 without isolated vertices does the following statement hold? Let $s, t, k, m$ be positive integers with $\min \{s, t\} \gg m \gg k$ and $m \geq|E(H)|$. There is a $k$-connected monochromatic subgraph of order at least $s+t-f(H, k, m)$ in every rainbow $H$-free exact $m$-edge-coloring of $K_{s, t}$, where $f(H, k, m)$ does not depend on $s$ or $t$.

Let $\mathscr{B}$ be the set of bipartite graphs $H$ satisfying the statement in Question 6.2. We shall prove the following result in Section 6.4.

Theorem 6.5. The set $\mathscr{B}$ consists of precisely $2 P_{3}, 2 K_{2} \cup K_{1,3}$ and their subgraphs of order at least 3 without isolated vertices.

Finally, instead of looking for monochromatic subgraphs, we try to force large 2-colored subgraphs in Gallai-3-colorings. In [75], Fox, Grinshpun and Pach proved that every Gallai-3-coloring of $K_{n}$ contains a 2-colored complete graph of order $\Omega\left(n^{1 / 3} \log ^{2} n\right)$ (see Theorem 5.3). In [170], Wagner proved that every Gallai-3-coloring of $K_{n}$ contains a 2-colored subgraph with chromatic number at least $n^{2 / 3}$. In the final part of this chapter, we consider large $k$-connected 2-colored subgraphs in Gallai-3-colorings.

By the following example we know that such a largest $k$-connected 2colored subgraph has order at most $n-\lfloor(k-1) / 2\rfloor$. Let $G$ be a copy of $K_{n}$ with $V(G)=V_{1} \cup V_{2} \cup V_{3}$ such that $\left|V_{1}\right|=n-k+1,\left|V_{2}\right|=\lceil(k-1) / 2\rceil$ and $\left|V_{3}\right|=\lfloor(k-1) / 2\rfloor$. We color the edges of $G$ such that the edges between $V_{2}$ and $V_{1} \cup V_{3}$ are red, the edges between $V_{1}$ and $V_{3}$ are blue, and all the remaining edges are green. It is easy to see that $G$ is a Gallai-3-coloring of $K_{n}$, and the largest $k$-connected 2-colored subgraph has order $n-\lfloor(k-1) / 2\rfloor$. In light of this example, we may consider the following question.

Question 6.3. For $k \geq 1$ and sufficiently large $n$, does every Gallai-3-coloring of $K_{n}$ contain a $k$-connected 2-colored subgraph of order at least $n-L(k-$ $1) / 2\rfloor$ ?

In Section 6.5, we deal with this question. We prove that Question 6.3 has an affirmative answer for $1 \leq k \leq 3$, and give a counterexample to show 
that this question has a negative answer when $k=4 t$, where $t$ is any positive integer. We also provide a conjecture related to Question 6.3, and prove some special cases of this conjecture.

\subsection{A characterization of the set $\mathscr{H}$}

We begin with the following lemmas and corollary which will be used in our proofs.

Lemma 6.6 (Fujita and Magnant [81]). Let $m \geq 3$ and $k \geq 2$. If $n \geq$ $(m+11)(k-1)+7 k \ln k$, then every Gallai-m-coloring of $K_{n}$ contains a $k$ connected monochromatic subgraph of order at least $n-m(k-1)$.

Lemma 6.7 (Fujita and Magnant [81]). For $G \in\left\{K_{1,3}, P_{4}^{+}\right\}$, if $m \geq 4, k \geq 1$ and $n \geq 7.5(k-1)$, then every rainbow $G$-free exact m-edge-coloring of $K_{n}$ contains a $k$-connected monochromatic subgraph of order at least $n-3 k+2$.

Lemma 6.8 (Fujita and Magnant [81]). Let $k \geq 1$ and $m \geq \max \left\{\frac{k}{2}+8,15\right\}$. If $n \geq(m+11)(k-1)+7 k \ln k+2 m+3$, then every rainbow $P_{6}$-free exact m-edge-coloring of $K_{n}$ contains a $k$-connected monochromatic subgraph of order at least $n-7 k+2$.

Corollary 6.9. Let $k \geq 2$ and $m \geq \max \left\{\frac{k}{2}+8,44\right\}$. For every $H \in\left\{K_{2} \cup\right.$ $\left.K_{3}, K_{2} \cup P_{4}^{+}, K_{2} \cup P_{5}, P_{3} \cup P_{4}\right\}$, if $n \geq(m+11)(k-1)+7 k \ln k+2 m+3$, then every rainbow $H$-free exact $m$-edge-coloring of $K_{n}$ contains an ASMS $(k)$ (that is, $\left.H \in \mathscr{H}^{(2)}\right)$.

Proof. For any $H \in\left\{K_{2} \cup K_{3}, K_{2} \cup P_{4}^{+}, K_{2} \cup P_{5}, P_{3} \cup P_{4}\right\}$, let $G$ be an exact $m$-edge-coloring of $K_{n}$ without a rainbow $H$, and suppose that there exists no $\operatorname{ASMS}(k)$.

Firstly, we consider the case $H=K_{2} \cup K_{3}$. By Lemma 6.6, there is a rainbow $K_{3}$ in $G$, say with vertex set $V$. Then $C(V(G) \backslash V) \subseteq C(V)$ for avoiding a rainbow $K_{2} \cup K_{3}$. If $|C(V(G) \backslash V)| \leq 2$, then there is an $A S M S(k)$ by Theorem 6.1. Thus $|C(V(G) \backslash V)|=|C(V)|=3$. Since $|V(G) \backslash V| \geq$ $(m+11)(k-1)+7 k \ln k+2 m$, there is also a rainbow $K_{3}$ in $G[V(G) \backslash V]$ by Lemma 6.6, say with vertex set $V^{\prime}$. Since the number of colors used in $G$ is 
at least 44 , there is an edge $e$ with $c(e) \geq 4$ such that $e$ is incident with some vertex in $V(G) \backslash\left(V \cup V^{\prime}\right)$. Then there is a rainbow $K_{2} \cup K_{3}$, a contradiction.

Secondly, we consider the case $H=K_{2} \cup P_{4}^{+}$. By Lemma 6.7, there is a rainbow $P_{4}^{+}$in $G$, say with vertex set $U=\left\{u_{1}, u_{2}, \ldots, u_{5}\right\}$ such that $c\left(u_{1} u_{2}\right)=$ $1, c\left(u_{2} u_{3}\right)=2, c\left(u_{3} u_{4}\right)=3$ and $c\left(u_{3} u_{5}\right)=4$. Then $C(V(G) \backslash U) \subseteq\{1,2,3,4\}$ for avoiding a rainbow $K_{2} \cup P_{4}^{+}$. If $|C(V(G) \backslash U)| \leq 2$, then there is an $A S M S(k)$ by Theorem 6.1, a contradiction. If $|C(V(G) \backslash U)|=4$, then by Lemma 6.7, there is also a rainbow $P_{4}^{+}$in $G[V(G) \backslash U]$, say with vertex set $U^{\prime}$. Since the number of colors used in $G$ is at least 44 , there is an edge $e$ with $c(e) \geq 5$ such that $e$ is incident with some vertex in $V(G) \backslash\left(U \cup U^{\prime}\right)$. Then there is a rainbow $K_{2} \cup P_{4}^{+}$, a contradiction. Thus $|C(V(G) \backslash U)|=3$. So we may assume that $C(V(G) \backslash U)=\left\{c_{1}, c_{2}, c_{3}\right\} \subset\{1,2,3,4\}$ and $c\left(e_{i}\right)=c_{i}$ for $i \in[3]$, where $e_{1}, e_{2}$ and $e_{3}$ are three edges in $G[V(G) \backslash U]$. Let $U^{\prime \prime}$ be the set of vertices incident with at least one of $e_{1}, e_{2}$ and $e_{3}$, so $\left|U^{\prime \prime}\right| \leq 6$. Since $m-\left(|U|\left|U^{\prime \prime}\right|+\left(\begin{array}{c}|U| \\ 2\end{array}\right)\right) \geq 4$, we have $\left|C\left(U, V(G) \backslash\left(U \cup U^{\prime \prime}\right)\right) \cap\{5,6, \ldots, m\}\right| \geq 4$. It is easy to check that there is a rainbow $K_{2} \cup P_{4}^{+}$, a contradiction.

Finally, we consider the cases $H=K_{2} \cup P_{5}$ and $H=P_{3} \cup P_{4}$. By Lemma 6.8, there is a rainbow $P_{6}$ in $G$, say with vertex set $W=\left\{w_{1}, w_{2}, \ldots, w_{6}\right\}$ such that $c\left(w_{i} w_{i+1}\right)=i$ for each $i \in[5]$. When $H=K_{2} \cup P_{5}$, we have $C(V(G) \backslash W) \subseteq$ $\{2,3,4\}$ for avoiding a rainbow $K_{2} \cup P_{5}$, and moreover we have $C\left(\left\{w_{1}, w_{3}, w_{4}\right.\right.$, $\left.\left.w_{6}\right\}, V(G) \backslash W\right) \subseteq\{1,2, \ldots, 5\}$ for the same reason. Thus we may assume that $c\left(w_{2} w^{\prime}\right)=6$ for some $w^{\prime} \in V(G) \backslash W$. In order to avoid a rainbow $K_{2} \cup P_{5}$, we have $C\left(w^{\prime}, V(G) \backslash\left(W \cup\left\{w^{\prime}\right\}\right)\right) \subseteq\{2,3\}$, and we further have $C\left(V(G) \backslash\left(W \cup\left\{w^{\prime}\right\}\right)\right) \subseteq\{2,3\}$. By Theorem 6.1, there is an $\operatorname{ASMS}(k)$, a contradiction.

When $H=P_{3} \cup P_{4}$, we have $C(W, V(G) \backslash W) \subseteq\{1,2, \ldots, 5\}$ for avoiding a rainbow $P_{3} \cup P_{4}$. Thus we may assume that $c(x y)=6$, where $x$ and $y$ are two distinct vertices in $V(G) \backslash W$. If $c\left(w_{2} x\right)=1$, then $w_{2} x y$ and $w_{3} w_{4} w_{5} w_{6}$ form a rainbow $P_{3} \cup P_{4}$, a contradiction. If $c\left(w_{2} x\right)=5$ (resp., $c\left(w_{2} x\right) \in$ $\{2,3\}$ ), then $w_{3} w_{4} w_{5}$ (resp., $w_{4} w_{5} w_{6}$ ) and $w_{1} w_{2} x y$ form a rainbow $P_{3} \cup P_{4}$, a contradiction. Thus $c\left(w_{2} x\right)=4$, and by symmetry we have $c\left(w_{2} y\right)=4$, $c\left(w_{5} x\right)=c\left(w_{5} y\right)=2$. For any vertex $z \in V(G) \backslash(W \cup\{x, y\})$, if $c(x z) \notin\{3,6\}$, then it is easy to find a rainbow $P_{3} \cup P_{4}$. If $c(x z) \in\{3,6\}$, then $w_{1} w_{2} x z$ and 
$y w_{5} w_{6}$ form a rainbow $P_{3} \cup P_{4}$, a contradiction.

Note that if $G$ and $H$ are two graphs with $G \subseteq H$, then any rainbow $G$-free edge-coloring of $K_{n}$ certainly contains no rainbow $H$. Thus we have the following simple result.

Fact 6.1. Let $G$ and $H$ be two graphs of order at least 3 without isolated vertices. If $G \subseteq H$, then $G \notin \mathscr{G} \cup \mathscr{H}$ implies $H \notin \mathscr{H}$.

Now we give a complete characterization of $\mathscr{H}^{(2)}$.

Theorem 6.10. The set $\mathscr{H}^{(2)}$ consists of precisely $P_{3} \cup P_{4}, K_{2} \cup P_{5}, K_{2} \cup K_{3}$, $K_{2} \cup P_{4}^{+}$and their subgraphs of order at least 3 with component number 2.

Proof. In the following, we will prove that $\mathscr{H}^{(2)}$ is a subset of $P_{3} \cup P_{4}, K_{2} \cup P_{5}$, $K_{2} \cup K_{3}, K_{2} \cup P_{4}^{+}$and their subgraphs of order at least 3 with component number 2 . This together with Corollary 6.9 and Fact 6.1 completes the proof of Theorem 6.10.

For the proof, we will consider two edge-colorings $R_{1}$ and $R_{2}$ (see Figure 6.1) constructed as follows. Let $R$ be an m-edge-coloring of $K_{n}$ with $V(R)=V_{1} \cup V_{2} \cup V_{3}$, such that each of $V_{1}, V_{2}$ and $V_{3}$ contains about $n / 3$ vertices. We color the edges such that $C\left(V_{1}, V_{2}\right)=\{1\}, C\left(V_{2}\right)=C\left(V_{2}, V_{3}\right)=\{2\}$, $C\left(V_{3}\right)=C\left(V_{3}, V_{1}\right)=\{3\}$, and all the edges within $V_{1}$ are colored with color 1 except for a rainbow matching (resp., rainbow star) using all the remaining colors, and let $R_{1}$ (resp., $R_{2}$ ) be the resulting edge-coloring of $K_{n}$. Note that both the largest monochromatic $k$-connected subgraphs of $R_{1}$ and $R_{2}$ have order about $2 n / 3$, so neither $R_{1}$ nor $R_{2}$ contains an $\operatorname{ASMS}(k)$. Thus every graph in $\mathscr{H}^{(2)}$ should be a rainbow subgraph of both $R_{1}$ and $R_{2}$.

Recall that we have $\mathscr{G}=\left\{K_{3}, P_{3}, P_{4}, P_{5}, P_{6}, K_{1,3}, P_{4}^{+}\right\}$(see Theorem 6.2). By Fact 6.1, each graph in $\mathscr{H}$ is a union of graphs in $\mathscr{G} \cup\left\{K_{2}\right\}$. In the rest of the proof, we consider six types of graphs that are possible in $\mathscr{H}^{(2)}$ (note that some graphs might be contained in two types).

Type 1. $K_{2} \cup G$, where $G \in \mathscr{G} \cup\left\{K_{2}\right\}$;

Type 2. $P_{6} \cup G$, where $G \in \mathscr{G}$;

Type 3. $K_{3} \cup G$, where $G \in \mathscr{G}$;

Type 4. $P_{5} \cup G$, where $G \in \mathscr{G}$; 

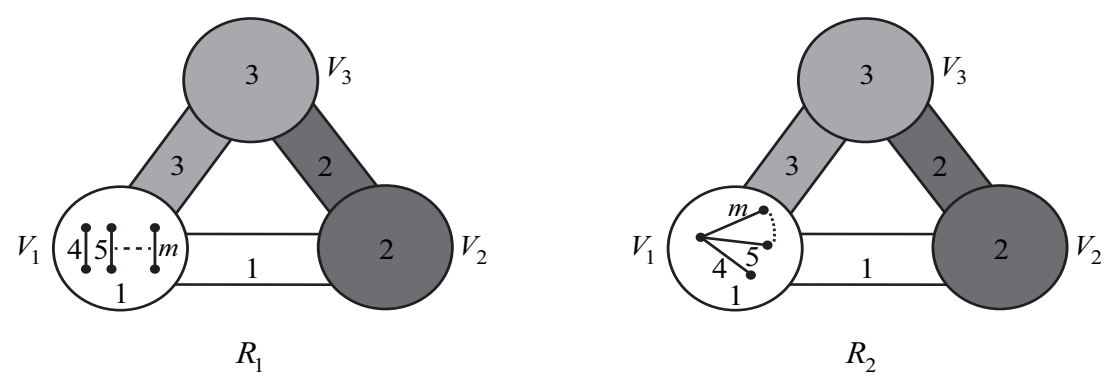

Figure 6.1: The edge-colorings $R_{1}$ and $R_{2}$.

Type 5. $K_{1,3} \cup G$ and $P_{4}^{+} \cup G$, where $G \in \mathscr{G}$;

Type 6. $P_{3} \cup G$ and $P_{4} \cup G$, where $G \in \mathscr{G}$.

Since every rainbow $K_{2} \cup P_{6}$ contains six distinct colors (at least three colors in $\{4,5, \ldots, m\}$ ), there is no rainbow $K_{2} \cup P_{6}$ in $R_{2}$. Thus $K_{2} \cup P_{6} \notin \mathscr{H}^{(2)}$ and there is no graph of Type 2 that is possible in $\mathscr{H}^{(2)}$. For Type $1, K_{2} \cup K_{3}$, $K_{2} \cup P_{5}, K_{2} \cup P_{4}^{+}$along with their subgraphs of order at least 3 and component number 2 are possible in $\mathscr{H}^{(2)}$.

Note that in $R_{1}$, every rainbow $K_{3}$ is colored by colors 1,2 and 3, every rainbow $K_{1,3}$ contains colors 1 and 3 , every rainbow $P_{5}$ contains colors 1 and 3, and every rainbow $P_{3}$ contains an edge with color 1 or color 3 . Thus $K_{3} \cup P_{3}, K_{1,3} \cup P_{3}, P_{4}^{+} \cup P_{3}, P_{5} \cup P_{3} \notin \mathscr{H}^{(2)}$, so there is no graph of Types 3,4 and 5 that is possible in $\mathscr{H}^{(2)}$. For the same reason with $K_{2} \cup P_{6}$, we have $2 P_{4} \notin \mathscr{H}^{(2)}$. Thus only $2 P_{3}$ and $P_{3} \cup P_{4}$ of Type 6 are possible in $\mathscr{H}^{(2)}$.

In the following, we consider the set $\mathscr{H}$.

Lemma 6.11. The set $\mathscr{H}$ is a subset of $P_{3} \cup P_{4}, K_{2} \cup P_{5}, K_{2} \cup 2 P_{3}, 2 K_{2} \cup K_{3}$, $2 K_{2} \cup P_{4}^{+}, 3 K_{2} \cup K_{1,3}$ and their disconnected subgraphs of order at least 3.

Proof. By Fact 6.1, each graph in $\mathscr{H}$ is a union of graphs in $\mathscr{G} \cup\left\{K_{2}\right\}$. Thus for every $H \in \mathscr{H}$, we have $\Delta(H) \leq 3$. Using the edge-coloring $R_{2}$ constructed in the proof of Theorem 6.10, we can deduce that

- $H$ has at most four components,

and further that 
- if $\Delta(H)=1$, then $e(H) \leq 4$; if $\Delta(H)=2$, then $e(H) \leq 5$; if $\Delta(H)=3$, then $e(H) \leq 6$.

Moreover, by Fact 6.1 we have that for every $H \in \mathscr{H}$,

- for any two components $C_{1}$ and $C_{2}$ of $H, C_{1} \cup C_{2} \in \mathscr{H}^{(2)}$.

Now for every graph $H^{\prime} \in \mathscr{H}^{(2)}=\left\{P_{3} \cup P_{4}, 2 P_{3}, K_{2} \cup P_{4}, K_{2} \cup P_{3}, 2 K_{2}, K_{2} \cup\right.$ $\left.P_{5}, K_{2} \cup K_{3}, K_{2} \cup P_{4}^{+}, K_{2} \cup K_{1,3}\right\}$, we can construct graphs that are possible in $\mathscr{H}$ by adding components to $H^{\prime}$ subject to the properties shown above. This completes the proof.

Lemma 6.12. Let $k \geq 2$ and $m \geq \max \left\{\frac{k}{2}+8,77\right\}$. For every $H \in\left\{K_{2} \cup 2 P_{3}\right.$, $\left.2 K_{2} \cup K_{3}, 2 K_{2} \cup P_{4}^{+}, 3 K_{2} \cup K_{1,3}\right\}$, if $n \geq(m+11)(k-1)+7 k \ln k+2 m+3$, then every rainbow $H$-free exact m-edge-coloring of $K_{n}$ contains an ASMS $(k)$ (that is, $H \in \mathscr{H}$ ).

Proof. For any $H \in\left\{K_{2} \cup 2 P_{3}, 2 K_{2} \cup K_{3}, 2 K_{2} \cup P_{4}^{+}, 3 K_{2} \cup K_{1,3}\right\}$, let $G$ be a rainbow $H$-free exact $m$-edge-coloring of $K_{n}$, and suppose that there exists no $\operatorname{ASMS}(k)$.

Firstly, we consider the case $H=K_{2} \cup 2 P_{3}$. By Lemma 6.8, there is a rainbow $P_{6}$ in $G$, say with vertex set $U=\left\{u_{1}, u_{2}, \ldots, u_{6}\right\}$ such that $c\left(u_{i} u_{i+1}\right)=$ $i$ for each $i \in[5]$. For avoiding a rainbow $K_{2} \cup 2 P_{3}$, we have $C(V(G) \backslash$ $U) \subseteq\{1,2,4,5\}$ and $\left|C\left(u_{i}, V(G) \backslash U\right) \cap\{6,7, \ldots, m\}\right| \leq 1$ for each $i \in\{3,4\}$. Moreover, if there exist two vertices $x, y \in V(G) \backslash U$ with $c\left(x u_{1}\right), c\left(y u_{1}\right) \in$ $\{6,7, \ldots, m\}$ and $c\left(x u_{1}\right) \neq c\left(y u_{1}\right)$, then $x u_{1} y, u_{2} u_{3} u_{4}$ and $u_{5} u_{6}$ form a rainbow $K_{2} \cup 2 P_{3}$, a contradiction. Thus $\left|C\left(u_{1}, V(G) \backslash U\right) \cap\{6,7, \ldots, m\}\right| \leq 1$, and by symmetry we have $\left|C\left(u_{6}, V(G) \backslash U\right) \cap\{6,7, \ldots, m\}\right| \leq 1$. Since there are at least 77 colors used in $G$, we have $C\left(\left\{u_{2}, u_{5}\right\}, V(G) \backslash U\right) \cap\{6,7, \ldots, m\} \neq \emptyset$, say $c\left(u_{2} u\right)=m$ for some $u \in V(G) \backslash U$. In order to avoid a rainbow $K_{2} \cup 2 P_{3}$, we have that $G[V(G) \backslash(U \cup\{u\})]$ is a monochromatic subgraph using color 4, contradicting the assumption that $G$ contains no ASMS $(k)$.

Secondly, we consider the case $H=2 K_{2} \cup K_{3}$. By Corollary 6.9, there is a rainbow $K_{2} \cup K_{3}$ in $G$, say with vertex set $V=\left\{v_{1}, v_{2}, v_{3}, v_{4}, v_{5}\right\}$ such that $c\left(v_{1} v_{2}\right)=1, c\left(v_{2} v_{3}\right)=2, c\left(v_{3} v_{1}\right)=3$ and $c\left(v_{4} v_{5}\right)=4$. Then $C(V(G) \backslash V) \subseteq$ $\{1,2,3,4\}$ to avoid a rainbow $2 K_{2} \cup K_{3}$. We first suppose that $C\left(\left\{v_{4}, v_{5}\right\}, V(G) \backslash\right.$ 
$V) \cap\{5,6, \ldots, m\} \neq \emptyset$, say $c\left(v_{4} v\right)=5$ for some $v \in V(G) \backslash V$. Then $C(V(G) \backslash$ $(V \cup\{v\})) \subseteq\{1,2,3\}$, and by Theorem 6.1 we have $C(V(G) \backslash(V \cup\{v\}))=$ $\{1,2,3\}$. By Lemma 6.6, $G[V(G) \backslash(V \cup\{v\})]$ contains a rainbow $K_{3}$, say with vertex set $V^{\prime}$. In order to avoid a rainbow $2 K_{2} \cup K_{3}$, we have $C\left(V^{\prime} \cup\right.$ $\left.\{v\} \cup\left(V \backslash\left\{v_{4}\right\}\right), V(G) \backslash\left(V \cup V^{\prime} \cup\{v\}\right)\right) \subseteq\{1,2,3,4,5\}$. Since the number of colors used in $G$ is at least 77, which is greater than $\left(\left|V \cup V^{\prime} \cup\{v\}\right|\right)$, we may further assume that $c\left(v_{4} v^{\prime}\right)=m$ for some $v^{\prime} \in V(G) \backslash\left(V \cup V^{\prime} \cup\{v\}\right)$. But then $c\left(v v^{\prime}\right) \notin[m]$, a contradiction. Therefore, $C\left(\left\{v_{4}, v_{5}\right\}, V(G) \backslash V\right) \cap$ $\{5,6, \ldots, m\}=\emptyset$. Since there are at least 77 colors used in $G$, we may assume that $\left|C\left(v_{1}, V(G) \backslash V\right) \cap\{5,6, \ldots, m\}\right| \geq 2$, say $c\left(v_{1} v^{\prime \prime}\right)=5$ and $c\left(v_{1} v^{\prime \prime \prime}\right)=6$ for some $v^{\prime \prime}, v^{\prime \prime \prime} \in V(G) \backslash V$. Then $c\left(v^{\prime \prime} v^{\prime \prime \prime}\right) \in\{2,4\}$, and we thus have $C\left(V(G) \backslash\left(V \cup\left\{v^{\prime \prime}, v^{\prime \prime \prime}\right\}\right)\right) \subseteq\{2,4\}$. By Theorem 6.1, there is an ASMS $(k)$, a contradiction.

Next, we consider the case $H=2 K_{2} \cup P_{4}^{+}$. By Corollary 6.9, there is a rainbow $K_{2} \cup P_{4}^{+}$in $G$, say with vertex set $W=\left\{w_{1}, w_{2}, \ldots, w_{7}\right\}$ such that $c\left(w_{1} w_{2}\right)=1, c\left(w_{2} w_{3}\right)=2, c\left(w_{3} w_{4}\right)=3, c\left(w_{3} w_{5}\right)=4$ and $c\left(w_{6} w_{7}\right)=5$. Then $C(V(G) \backslash W) \subseteq\{1,2, \ldots, 5\}$ for avoiding a rainbow $2 K_{2} \cup P_{4}^{+}$. We first suppose that $C\left(\left\{w_{6}, w_{7}\right\}, V(G) \backslash W\right) \cap\{6,7, \ldots, m\} \neq \emptyset$, say $c\left(w_{6} w\right)=6$ for some $w \in V(G) \backslash W$. Then $C(V(G) \backslash(W \cup\{w\})) \subseteq\{1,2,3,4\}$, and by Theorem 6.1 we have $|C(V(G) \backslash(W \cup\{w\}))| \geq 3$. Let $c_{1}, c_{2}, c_{3}$ be three distinct colors used in $G[V(G) \backslash(W \cup\{w\})]$ and let $c\left(e_{i}\right)=c_{i}$ for $1 \leq i \leq 3$, where $e_{1}, e_{2}$ and $e_{3}$ are three edges in $G[V(G) \backslash(W \cup\{w\})]$. Let $W^{\prime}$ be the set of vertices incident with at least one of $e_{1}, e_{2}$ and $e_{3}$, so $\left|W^{\prime}\right| \leq 6$. Let $A=V(G) \backslash\left(W \cup W^{\prime} \cup\{w\}\right)$. In order to avoid a rainbow $2 K_{2} \cup P_{4}^{+}$, we have $\left|C\left(w_{i}, A\right) \cap\{7,8, \ldots, m\}\right| \leq 1$ for each $w_{i} \in W \backslash\left\{w_{6}\right\}$. Since the number of colors used in $G$ is at least 77, which is greater than $\left(\begin{array}{c}|W \cup\{w\}| \\ 2\end{array}\right)+|W|\left|W^{\prime}\right|+$ $\left|W \backslash\left\{w_{6}\right\}\right|$, we may further assume that $c\left(w_{6} w^{\prime}\right)=m$ for some vertex $w^{\prime} \in A$. Let $w^{\prime \prime} \in A \backslash\left\{w^{\prime}\right\}$. Recall that $c\left(w^{\prime} w^{\prime \prime}\right) \in C(V(G) \backslash(W \cup\{w\})) \subseteq\{1,2,3,4\}$. Thus $\left\{w_{6}, w_{7}, w, w^{\prime}, w^{\prime \prime}\right\}$ forms a rainbow $P_{4}^{+}$, which together with some edge within $\left\{w_{1}, w_{2}, \ldots, w_{5}\right\}$ and some edge within $W^{\prime}$ forms a rainbow $2 K_{2} \cup P_{4}^{+}$, a contradiction.

Therefore, $C\left(\left\{w_{6}, w_{7}\right\}, V(G) \backslash W\right) \cap\{6,7, \ldots, m\}=\emptyset$. Thus every edge with color in $\{6,7, \ldots, m\}$ is incident with a vertex in $\left\{w_{1}, w_{2}, \ldots, w_{5}\right\}$. If $C(V(G) \backslash$ $W) \cap\{3,4\} \neq \emptyset$, say $c(a b)=3$ for some $a, b \in V(G) \backslash W$, then $\mid C\left(w_{i}, V(G) \backslash\right.$ 
$(W \cup\{a, b\})) \cap\{6,7, \ldots, m\} \mid \leq 1$ for $i \in[5]$. Thus $|C(G)| \leq 5+\left(\begin{array}{c}|W| \\ 2\end{array}\right)<m$, a contradiction. Thus $C(V(G) \backslash W)=\{1,2,5\}$. Let $c\left(f_{1}\right)=1, c\left(f_{2}\right)=2$ and $c\left(f_{3}\right)=5$, where $f_{1}, f_{2}$ and $f_{3}$ are three edges in $G[V(G) \backslash W]$. Let $W^{\prime \prime}$ be the set of vertices incident with at least one of $f_{1}, f_{2}$ and $f_{3}$. For avoiding a rainbow $2 K_{2} \cup P_{4}^{+}$, we have $\left|C\left(w_{i}, V(G) \backslash\left(W \cup W^{\prime \prime}\right)\right) \cap\{6,7, \ldots, m\}\right| \leq 1$ for $i \in\{2,4,5\}$. For $j \in\{1,3\}$, if $\left|C\left(w_{j}, V(G) \backslash\left(W \cup W^{\prime \prime}\right)\right) \cap\{6,7, \ldots, m\}\right| \geq 3$, say $c\left(w_{j} x_{1}\right)=6, c\left(w_{j} x_{2}\right)=7$ and $c\left(w_{j} x_{3}\right)=8$, then for any vertex $y \in$ $V(G) \backslash\left(W \cup W^{\prime \prime} \cup\left\{x_{1}, x_{2}, x_{3}\right\}\right)$, we have $c\left(x_{1} y\right) \notin[m]$, a contradiction. Thus $|C(G)| \leq 3+2 \cdot 2+\left(\begin{array}{c}|W| \\ 2\end{array}\right)<m$, a contradiction.

Finally, we consider the case $H=3 K_{2} \cup K_{1,3}$. From the above argument, there is a rainbow $2 K_{2} \cup P_{4}^{+}$in $G$, say with vertex set $B$ and color set $\{1,2, \ldots, 6\}$. In order to avoid a rainbow $3 K_{2} \cup K_{1,3}$, we have $C(V(G) \backslash B) \subseteq$ $\{1,2, \ldots, 6\}$ and $|C(b, V(G) \backslash B) \cap\{7,8, \ldots, m\}| \leq 1$ for any vertex $b \in B$. Thus $|C(G)| \leq|B|+\left(\begin{array}{c}|B| \\ 2\end{array}\right)<m$, a contradiction.

From Corollary 6.9, Fact 6.1, Lemmas 6.11 and 6.12, we conclude that the statement of Theorem 6.3 is valid.

\subsection{Proof of Theorem 6.4}

We first state some known results which will be used in our proofs.

Lemma 6.13 (Gyárfás [90]). Every m-edge-coloring of $K_{n}$ has a monochromatic connected subgraph with at least $\frac{n}{m-1}$ vertices.

Lemma 6.14 (Liu, Morris and Prince [125]). Every m-edge-coloring of $K_{s, t}$ has a monochromatic component of order at least $\frac{s+t}{m}$.

Lemma 6.15 (Mader [134]). Let $d$ be a real number, and let $G$ be a graph with average degree $d$. Then $G$ has a $\frac{d}{4}$-connected subgraph $H$, and therefore $|V(H)| \geq \frac{d}{4}$.

Lemma 6.16 (Liu, Morris and Prince [125]). Let $n, m, k$ be positive integers and $\epsilon>0$ satisfying $m \geq 3$ and $n \geq \frac{11(2+\epsilon)}{\epsilon} k^{2} m^{2}$. Then in any m-edgecoloring of $K_{n}$, there is a k-connected monochromatic subgraph of order at least $\frac{n}{m-1}-\left(1+\frac{1}{m(m-2)}+\epsilon\right) k^{2} m$. 
We have the following simple corollary that is obtained by setting $\epsilon=\frac{1}{4}$ in Lemma 6.16.

Corollary 6.17. If $m \geq 3, k \geq 2$ and $n \geq 99 k^{2} m^{2}$, then in any m-edgecoloring of $K_{n}$, there is a $k$-connected monochromatic subgraph of order at least $\frac{n}{m-1}-\frac{19}{12} k^{2} m$.

In the following, we first consider the case $k=1$ in Theorem 6.4.

Theorem 6.18. For any graph $H \in \mathscr{G} \cup \mathscr{H}$, if $m \geq 77$ and $n \geq 4 m$, then any rainbow $\left(P_{3} \cup H\right)$-free exact $m$-edge-coloring of $K_{n}$ contains a connected monochromatic subgraph of order at least $\frac{n-|V(H)|}{|E(H)|}$.

Proof. For any $H \in \mathscr{G} \cup \mathscr{H}$, let $G$ be a rainbow $\left(P_{3} \cup H\right)$-free exact $m$-edgecoloring of $K_{n}$ with $C(G)=[m]$. Suppose that $G$ contains no connected monochromatic subgraph of order at least $\frac{n-|V(H)|}{|E(H)|}$.

Since $H \subseteq \mathscr{G} \cup \mathscr{H}$, every rainbow $H$-free edge-coloring of $K_{n}$ contains an almost spanning 2-connected (and thus connected) monochromatic subgraph with order greater than $\frac{n-|V(H)|}{|E(H)|}$. Thus there is a rainbow $H$ in $G$, say with vertex set $V(H)$ and color set $C(H)=\{1,2, \ldots,|E(H)|\}$. Let $C^{\prime}=C(G) \backslash C(H)$. If $\left|C(G-V(H)) \cap C^{\prime}\right| \leq 1$ (that is, $\left.|C(G-V(H))| \leq|E(H)|+1\right)$, then there is a connected monochromatic subgraph of order at least $\frac{n-|V(H)|}{|E(H)|}$ by Lemma 6.13, a contradiction. Thus $\left|C(G-V(H)) \cap C^{\prime}\right| \geq 2$, say $m-1, m \in C(G-V(H))$. In order to avoid a rainbow $P_{3} \cup H$, for any two edges $e_{1}, e_{2} \in E(G-V(H))$ with $c\left(e_{1}\right) \neq c\left(e_{2}\right)$ and $c\left(e_{1}\right), c\left(e_{2}\right) \in C^{\prime}$, we have that $e_{1}$ and $e_{2}$ are nonadjacent. Let $U=\{v \in V(G-V(H)): v$ is incident with an edge of color $m$ in $G-V(H)\}$ and $V=V(G-V(H)) \backslash U$. Note that we have $U \neq \emptyset$ and $V \neq \emptyset$. Then $C(U, V) \subseteq C(H)$, and there is a connected monochromatic subgraph of order at least $\frac{|U|+|V|}{|E(H)|}=\frac{n-|V(H)|}{|E(H)|}$ by Lemma 6.14, a contradiction.

Next we consider the case $k \geq 2$ in Theorem 6.4.

Theorem 6.19. For any graph $H \in \mathscr{G} \cup \mathscr{H}$, if $k \geq 2, m \geq \max \left\{\frac{k}{2}+8,77\right\}$ and $n \geq 12 \cdot 99 \mathrm{k}^{2} \mathrm{~m}^{2}$, then any rainbow $\left(P_{3} \cup H\right)$-free exact m-edge-coloring of $K_{n}$ contains a k-connected monochromatic subgraph of order at least $\frac{n-|V(H)|}{13|E(H)|}$. 
Proof. For any $H \in \mathscr{G} \cup \mathscr{H}$, let $G$ be a rainbow $\left(P_{3} \cup H\right)$-free exact $m$-edgecoloring of $K_{n}$ with $C(G)=[m]$. Suppose that $G$ contains no $k$-connected monochromatic subgraph of order at least $\frac{n-|V(H)|}{13|E(H)|}$. Since $H \in \mathscr{G} \cup \mathscr{H}$, we may further assume that there is a rainbow $H$ in $G$; otherwise there would be an $A S M S(k)$ of order greater than $\frac{n-|V(H)|}{13|E(H)|}$. Consider a rainbow copy of $H$ in $G$ with vertex set $V(H)$ and color set $C(H)=\{1,2, \ldots,|E(H)|\}$. Let $C^{\prime}=C(G) \backslash C(H)=\{|E(H)|+1, \ldots, m\}$. Recall that for any $H \in \mathscr{G} \cup \mathscr{H}$, we have $|V(H)| \leq 10$ and $|E(H)| \leq 6$.

If $\left|C(G-V(H)) \cap C^{\prime}\right| \leq 1$ (that is, $|C(G-V(H))| \leq|E(H)|+1$ ), then by Corollary 6.17 there is a $k$-connected monochromatic subgraph of order at least $\frac{n-|V(H)|}{|E(H)|}-\frac{19(|E(H)|+1)}{12} \cdot k^{2}$, which is at least $\frac{n-|V(H)|}{13|E(H)|}$, a contradiction.

Thus $\left|C(G-V(H)) \cap C^{\prime}\right| \geq 2$. In order to avoid a rainbow $P_{3} \cup H$, for any two edges $e_{1}, e_{2} \in E(G-V(H))$ with $c\left(e_{1}\right) \neq c\left(e_{2}\right)$ and $c\left(e_{1}\right), c\left(e_{2}\right) \in C^{\prime}$, we have that $e_{1}$ and $e_{2}$ are non-adjacent. For every $|E(H)|+1 \leq i \leq m$, let $U_{i}=\{v \in V(G) \backslash V(H): v$ is incident with an edge of color $i$ in $G-V(H)\}$, and let $U_{m+1}=V(G-V(H)) \backslash\left(\bigcup_{i \in C^{\prime}} U_{i}\right)$. Since $\left|C(G-V(H)) \cap C^{\prime}\right| \geq 2$, at least two of the sets $U_{|E(H)|+1}, \ldots, U_{m}, U_{m+1}$ are non-empty. Let $U_{i_{1}}, \ldots, U_{i_{t}}$ be all the non-empty sets of $U_{|E(H)|+1}, \ldots, U_{m}, U_{m+1}$, where $2 \leq t \leq m-|E(H)|+1$. Note that all the edges between these $t$ sets are colored by colors in $C(H)$, and $C\left(U_{j}\right) \subseteq C(H) \cup\{j\}$ for all $j \in\left\{i_{1}, \ldots, i_{t}\right\}$. Then for each $j \in\left\{i_{1}, \ldots, i_{t}\right\}$, we have $\left|U_{j}\right| \leq\lfloor n / 12\rfloor-1$; otherwise there exists a $k$-connected monochromatic subgraph $F$ with $|V(F)| \geq \frac{\left|U_{j}\right|}{|E(H)|}-\frac{19(|E(H)|+1)}{12} \cdot k^{2} \geq \frac{\mid n / 12\rfloor}{|E(H)|}-\frac{19(|E(H)|+1)}{12} \cdot k^{2} \geq$ $\frac{n-|V(H)|}{13|E(H)|}$ by Corollary 6.17. If $t \leq 12$, then $|V(G-V(H))| \leq t \cdot(\lfloor n / 12\rfloor-1) \leq$ $12 \cdot(n / 12-1)=n-12<n-|V(H)|$, a contradiction. Thus $t \geq 13$.

We now choose a subset $L \subseteq\{1, \ldots, t\}$ such that $A=\bigcup_{\ell \in L} U_{i_{\ell}}$ and $B=$ $\bigcup_{h \in[t] \backslash L} U_{i_{h}}$ satisfy

(1) $|A| \geq|B|$;

(2) $|A|-|B|$ is minimum subject to (1).

Claim 6.1. $|A|-|B| \leq \frac{n-|V(H)|}{6}$.

Proof. If $|A|-|B| \geq\lfloor(n-|V(H)|) / 6\rfloor+1$, then for an arbitrary element $s \in L$, let $A^{\prime}=A \backslash U_{i_{s}}$ and $B^{\prime}=B \cup U_{i_{s}}$. Then $\left|A^{\prime}\right|-\left|B^{\prime}\right|=|A|-\left|U_{i_{s}}\right|-\left(|B|+\left|U_{i_{s}}\right|\right)=$ 
$|A|-|B|-2\left|U_{i_{s}}\right|$. Thus $\left|A^{\prime}\right|-\left|B^{\prime}\right| \geq|A|-|B|-2(n / 12-1)=|A|-|B|-n / 6+2 \geq$ $\lfloor(n-|V(H)|) / 6\rfloor+1-n / 6+2 \geq 0$ and $\left|A^{\prime}\right|-\left|B^{\prime}\right| \leq|A|-|B|-2<|A|-|B|$, contradicting the choice of $L$. Thus $|A|-|B| \leq\lfloor(n-|V(H)|) / 6\rfloor \leq(n-$ $|V(H)|) / 6$.

Since $0 \leq|A|-|B| \leq \frac{n-|V(H)|}{6}$ and $|A|+|B|=n-|V(H)|$, we have $|A||B| \geq$ $\frac{5(n-|V(H)|)}{12} \cdot \frac{7(n-|V(H)|)}{12}=\frac{35(n-|V(H)|)^{2}}{144}$. Thus there are at least $\frac{35(n-|V(H)|)^{2}}{144}$ edges between $A$ and $B$. Since all the edges between $A$ and $B$ are colored by colors in $C(H)$, there are at least $\frac{35(n-|V(H)|)^{2}}{144|E(H)|}$ edges using a single color, say color 1 . Consider the bipartite graph $H^{\prime}$ with bipartition $(A, B)$ and $E\left(H^{\prime}\right)=\{u v: u \in$ $A, v \in B, c(u v)=1\}$. The average degree $d$ of $H^{\prime}$ satisfies $d \geq \frac{2}{n-|V(H)|}$. $\frac{35(n-|V(H)|)^{2}}{144|E(H)|}=\frac{35(n-|V(H)|)}{72|E(H)|}$. By Lemma 6.15, there is a subgraph $H^{\prime \prime} \subseteq H^{\prime}$ such that $\kappa\left(H^{\prime \prime}\right) \geq \frac{1}{4} \cdot \frac{35(n-|V(H)|)}{72|E(H)|}>\frac{n-|V(H)|}{13|E(H)|}>k$. Then $H^{\prime \prime}$ is a $k$-connected monochromatic subgraph of order at least $\frac{n-|V(H)|}{13|E(H)|}$, a contradiction.

For every $H \in \mathscr{G} \cup \mathscr{H}$, set $\beta(H)=\frac{1}{14|E(H)|}$. Then Theorem 6.4 follows from Theorems 6.18 and 6.19 .

\subsection{A characterization of the set $\mathscr{B}$}

We begin with the following proposition.

Proposition 6.20. The set $\mathscr{B}$ is a subset of $2 P_{3}, 2 K_{2} \cup K_{1,3}$ and their subgraphs of order at least 3.

Proof. Let $K_{s, t}$ be a complete bipartite graph with $s$ vertices in one partite set $U$ and $t$ vertices in the other partite set $V$. We will consider three edge-colorings of $K_{s, t}$ (see Figure 6.2) constructed as follows.

The edge-coloring $A_{1}$ is an $m$-edge-coloring of $K_{s, t}$ with $U=U_{1} \cup U_{2} \cup \cdots \cup$ $U_{m}$ such that each of $U_{1}, U_{2}, \ldots, U_{m}$ contains about $s / m$ vertices. We color the edges such that $c\left(U_{i}, V\right)=i$ for $i \in[m]$. The edge-coloring $A_{2}$ is an m-edgecoloring of $K_{s, t}$ with $U=U_{1} \cup U_{2} \cup\{u\}$ such that each of $U_{1}, U_{2}$ contains about $(s-1) / 2$ vertices. We color the edges such that $c\left(U_{1}, V\right)=1, c\left(U_{2}, V\right)=2$ and $C(u, V)=\{1,2, \ldots, m\}$. The edge-coloring $A_{3}$ is an $m$-edge-coloring of 
$K_{s, t}$ with $U=U_{3} \cup U_{4} \cup \cdots \cup U_{m}$ and $V=V_{3} \cup V_{4} \cup \cdots \cup V_{m}$, such that each of $U_{3}, U_{4}, \ldots, U_{m}$ contains about $s /(m-2)$ vertices, and each of $V_{3}, V_{4}, \ldots, V_{m}$ contains about $t /(m-2)$ vertices. We color the edges such that $c\left(U_{i}, V_{i}\right)=i$ for every $3 \leq i \leq m, c\left(\bigcup_{j=3}^{r} U_{j}, \bigcup_{\ell=r+1}^{m} V_{\ell}\right)=c\left(\bigcup_{j=3}^{r} V_{j}, \bigcup_{\ell=r+1}^{m} U_{\ell}\right)=1$, and all the remaining edges are colored with color 2 , where $r=\lfloor(m+2) / 2\rfloor$.

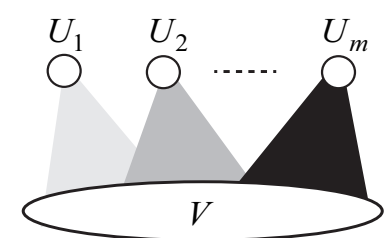

$A_{1}$

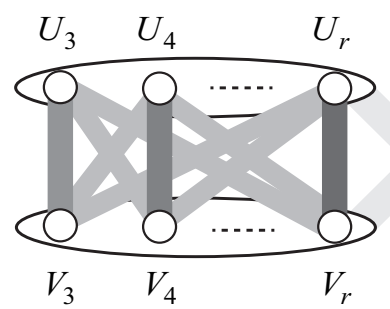

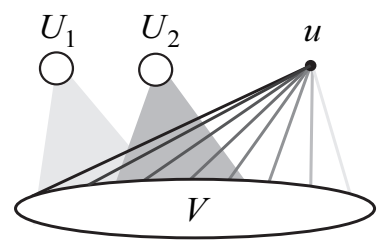

$A_{2}$

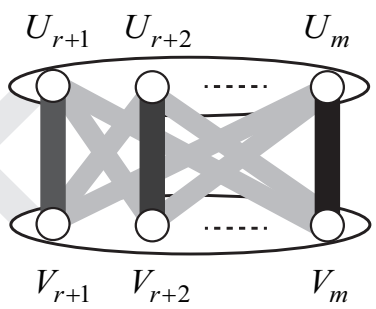

$A_{3}$

Figure 6.2: The edge-colorings $A_{1}, A_{2}$ and $A_{3}$.

Note that the largest monochromatic $k$-connected subgraphs of $A_{1}, A_{2}$ and $A_{3}$ have order about $s / m+t,(s-1) / 2+t$ and $(s+t) / 2$, respectively. Thus every graph in $\mathscr{B}$ must be a rainbow subgraph of $A_{1}, A_{2}$ and $A_{3}$.

For any graph $H \in \mathscr{B}$, since $A_{1}$ is rainbow $P_{4}$-free and $A_{3}$ is rainbow $K_{1,4}$ free, the longest path in $H$ has length at most 2 and $\Delta(H) \leq 3$. Thus each component of $H$ is a graph in $\left\{K_{2}, P_{3}, K_{1,3}\right\}$. Since $A_{2}$ is rainbow $4 K_{2}$-free, $H$ has at most 3 components. Moreover, every rainbow $K_{1,3}$ in $A_{3}$ uses both color 1 and color 2 , and every rainbow $P_{3}$ in $A_{3}$ uses at least one of color 1 and color 2. Thus $P_{3} \cup K_{1,3} \notin \mathscr{B}$. Furthermore, since $A_{2}$ is rainbow $\left(K_{2} \cup 2 P_{3}\right)$ free, we have $K_{2} \cup 2 P_{3} \notin \mathscr{B}$. From the above argument, we can deduce that $\mathscr{B} \subseteq\left\{P_{3}, K_{1,3}, 2 K_{2}, K_{2} \cup P_{3}, K_{2} \cup K_{1,3}, 2 P_{3}, 3 K_{2}, 2 K_{2} \cup P_{3}, 2 K_{2} \cup K_{1,3}\right\}$.

Next, we characterize the structure of edge-colored complete bipartite graphs without a rainbow $K_{1,3}$. 
Theorem 6.21. For $s \geq t \geq 3$, let $K_{s, t}$ be edge-colored such that it contains no rainbow $K_{1,3}$. Then, after renumbering the colors, one of the following holds:

(1) at most four colors are used;

(2) if $m$ is the number of colors used in the edge-coloring, then one partite set $U$ can be partitioned into $m-1$ non-empty parts $U_{2}, U_{3}, \ldots, U_{m}$, and the other partite set $V$ can be partitioned into $m-1$ non-empty parts $V_{2}, V_{3}, \ldots, V_{m}$, such that $C\left(U_{i}, V_{i}\right) \subseteq\{1, i\}$ for each $i \in\{2,3, \ldots, m\}$, and every other edge is colored by color 1 .

Proof. Let $G$ be a rainbow $K_{1,3}$-free edge-coloring of $K_{s, t}$ with partite sets $U=$ $\left\{u_{1}, u_{2}, \ldots, u_{s}\right\}$ and $V=\left\{v_{1}, v_{2}, \ldots, v_{t}\right\}$. Let $C(G)=[m]$. For a contradiction, suppose that there are at least five colors used in $G$ and (2) does not hold.

We call two adjacent edges of distinct colors a " $\wedge$ ". Note that every color appears on some $\wedge$. We claim that there are two $\wedge s$ with four distinct colors (possibly such two $\wedge s$ have common vertices, but no common edges). Indeed, since (2) does not hold, we may assume that colors 2 and 3 form a $\wedge$ without loss of generality. In order to avoid two $\wedge s$ with four distinct colors, each pair of colors in $C(G) \backslash\{2,3\}$ cannot form a $\wedge$. Let $c\left(u^{\prime} v^{\prime}\right)=c_{1}$, $c\left(u^{\prime \prime} v^{\prime \prime}\right)=c_{2}$, where $c_{1}, c_{2} \in C(G) \backslash\{2,3\}, c_{1} \neq c_{2}, u^{\prime}, u^{\prime \prime} \in U$ and $v^{\prime}, v^{\prime \prime} \in$ $V$. For avoiding two required $\wedge \mathrm{s}$, we have $c\left(u^{\prime} v^{\prime \prime}\right)=c\left(u^{\prime \prime} v^{\prime}\right)=2$ or 3 , say 2. So $C\left(\left\{u^{\prime}, v^{\prime}\right\}, V(G) \backslash\left\{u^{\prime}, v^{\prime}, u^{\prime \prime}, v^{\prime \prime}\right\}\right) \subseteq\left\{2, c_{1}\right\}$ and $C\left(\left\{u^{\prime \prime}, v^{\prime \prime}\right\}, V(G) \backslash\right.$ $\left.\left\{u^{\prime}, v^{\prime}, u^{\prime \prime}, v^{\prime \prime}\right\}\right) \subseteq\left\{2, c_{2}\right\}$. For any color $c \in C(G) \backslash\left\{2, c_{1}, c_{2}\right\}$ and an edge $u v$ with $c(u v)=c$, we have $u \in U \backslash\left\{u^{\prime}, u^{\prime \prime}\right\}$ and $v \in V \backslash\left\{v^{\prime}, v^{\prime \prime}\right\}$. Then $c\left(u,\left\{v^{\prime}, v^{\prime \prime}\right\}\right)=c\left(v,\left\{u^{\prime}, u^{\prime \prime}\right\}\right)=2$. Moreover, for any two distinct colors $c^{\prime}, c^{\prime \prime} \in$ $C(G) \backslash\{2\}$, the edge using color $c^{\prime}$ and the edge using color $c^{\prime \prime}$ are non-adjacent. Thus (2) holds if we exchange color 1 and color 2, a contradiction. Thus there are two $\wedge \mathrm{s}$ with four distinct colors. We may further consider four types of such two $\wedge$ s (see Figure 6.3).

Firstly, if there are two $\wedge$ s of type (A), then $c\left(u_{1} v_{3}\right) \notin[m]$ for avoiding a rainbow $K_{1,3}$, a contradiction. Thus there are no two $\wedge$ s of type (A). Secondly, we consider type (B). For avoiding two $\wedge$ s of type (A) and since $G$ is a rainbow $K_{1,3}$-free edge-coloring using at least five colors, we may assume that $c\left(u_{3} v_{5}\right)=5$. But then $c\left(u_{1} v_{5}\right) \notin[m]$, a contradiction. Thus there are no 


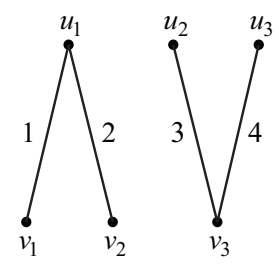

(A)

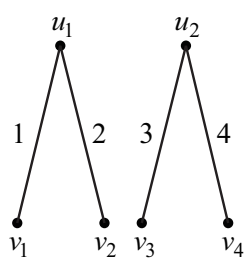

(B)

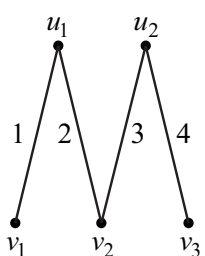

(C)

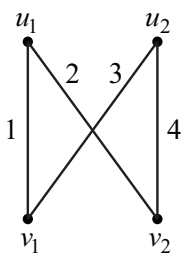

(D)

Figure 6.3: Four types of $\wedge$.

two $\wedge s$ of type (B), and there are no two $\wedge s$ of type (C) for the same reason. Finally, we consider type (D). For avoiding a rainbow $K_{1,3}$ and since there are at least five colors used in $G$, we may assume that $c\left(u_{3} v_{3}\right)=5$. But then $c\left(u_{1} v_{3}\right) \notin[m]$ for avoiding a rainbow $K_{1,3}$ and avoiding two $\wedge$ s of type (A), a contradiction. The result follows.

Corollary 6.22. Given positive integers $k, m, s, t$ with $m \geq k+4$ and $\min \{s, t\} \geq$ $m-1$, there is a spanning $k$-connected monochromatic subgraph in any rainbow $K_{1,3}$-free exact m-edge-coloring of $K_{s, t}$.

Proof. Let $G$ be a rainbow $K_{1,3}$-free exact $m$-edge-coloring of $K_{s, t}$ with partite sets $U$ and $V$. Since $m \geq k+4 \geq 5$ and by Theorem 6.21 , there is a partition $U_{2}, U_{3}, \ldots, U_{m}$ of $U$ and a partition $V_{2}, V_{3}, \ldots, V_{m}$ of $V$, such that $C\left(U_{i}, V_{i}\right) \subseteq$ $\{1, i\}$ for each $i \in\{2,3, \ldots, m\}$, and every other edge is colored by color 1 .

Let $H$ be the subgraph induced by color 1 . Then $|V(H)|=s+t$. For any vertex set $W$ of $k-1$ vertices $w_{1}, w_{2}, \ldots, w_{k-1} \in V(G)$, let $U_{i}^{\prime}=U_{i} \backslash W$ and $V_{i}^{\prime}=V_{i} \backslash W$ for every $i \in\{2,3, \ldots, m\}$. Since $m \geq k+4$, there are at least three distinct subindices $i_{1}, i_{2}, i_{3} \in\{2,3, \ldots, m\}$ and three distinct subindices $j_{1}, j_{2}, j_{3} \in\{2,3, \ldots, m\}$, such that $U_{\alpha}^{\prime}=U_{\alpha} \neq \emptyset$ and $V_{\beta}^{\prime}=V_{\beta} \neq \emptyset$ for each $\alpha \in\left\{i_{1}, i_{2}, i_{3}\right\}$ and each $\beta \in\left\{j_{1}, j_{2}, j_{3}\right\}$. Thus $H-W$ is connected, so $H$ is $k$-connected.

Lemma 6.23. Given positive integers $k, m, s, t$ with $m \geq \max \{k+4,7\}$ and $\min \{s, t\} \geq m$, there is a $k$-connected monochromatic subgraph of order at least $s+t-5$ in any rainbow $\left(2 P_{3}\right)$-free exact m-edge-coloring of $K_{s, t}$. 
Proof. Let $G$ be a rainbow $\left(2 P_{3}\right)$-free exact $m$-edge-coloring of $K_{s, t}$ with partite sets $U=\left\{u_{1}, u_{2}, \ldots, u_{s}\right\}$ and $V=\left\{v_{1}, v_{2}, \ldots, v_{t}\right\}$. For a contradiction, suppose that $G$ contains no $k$-connected monochromatic subgraph of order at least $s+t-5$. By Corollary 6.22, there is a rainbow $K_{1,3}$ in $G$, say $c\left(u_{1} v_{i}\right)=i$ for $1 \leq i \leq 3$.

For avoiding a rainbow $2 P_{3}$, we have $\left|C\left(v_{i}, U \backslash\left\{u_{1}\right\}\right) \cap\{4,5, \ldots, m\}\right| \leq 1$ for every $i \in[3]$. If $\left|C\left(u_{1}, V \backslash\left\{v_{1}, v_{2}, v_{3}\right\}\right) \cap\{4,5, \ldots, m\}\right| \geq 1$, say $c\left(u_{1} v_{4}\right)=4$, then $\left|C\left(V(G) \backslash\left\{u_{1}, v_{1}, v_{2}, v_{3}, v_{4}\right\}\right)\right|=1$ for avoiding a rainbow $2 P_{3}$, which implies that there is a $k$-connected monochromatic subgraph of order at least $s+t-5$, a contradiction. Thus $C\left(u_{1}, V \backslash\left\{v_{1}, v_{2}, v_{3}\right\}\right) \cap\{4,5, \ldots, m\}=\emptyset$. Since $|C(G)|=m \geq 7$, we may assume that $c\left(u_{2} v_{4}\right)=m$. Then $c\left(u_{2}, V \backslash\right.$ $\left.\left\{v_{1}, v_{2}, v_{3}\right\}\right)=c\left(v_{4}, U \backslash\left\{u_{1}\right\}\right)=m$ and thus $C\left(V(G) \backslash\left\{u_{1}, v_{1}, v_{2}, v_{3}\right\}\right)=\{m\}$, resulting in a $k$-connected monochromatic subgraph of order at least $s+t-4$, a contradiction.

Lemma 6.24. Given positive integers $k, m, s, t$ with $m \geq \max \{k+4,16\}$ and $\min \{s, t\} \geq m+1$, there is a $k$-connected monochromatic subgraph of order at least $s+t-6$ in any rainbow $\left(2 K_{2} \cup K_{1,3}\right)$-free exact m-edge-coloring of $K_{s, t}$.

Proof. Let $G$ be a rainbow $\left(2 K_{2} \cup K_{1,3}\right)$-free exact $m$-edge-coloring of $K_{s, t}$ with partite sets $U=\left\{u_{1}, u_{2}, \ldots, u_{s}\right\}$ and $V=\left\{v_{1}, v_{2}, \ldots, v_{t}\right\}$. For a contradiction, suppose that $G$ contains no $k$-connected monochromatic subgraph of order at least $s+t-6$. By Corollary 6.22, there is a rainbow $K_{1,3}$ in $G$, say $c\left(u_{1} v_{i}\right)=i$ for $1 \leq i \leq 3$.

If $\left|C\left(u_{1}, V \backslash\left\{v_{1}, v_{2}, v_{3}\right\}\right) \cap\{4,5, \ldots, m\}\right| \geq 2$, say $c\left(u_{1} v_{4}\right)=4$ and $c\left(u_{1} v_{5}\right)=$ 5, then $\left|C\left(V(G) \backslash\left\{u_{1}, v_{1}, v_{2}, \ldots, v_{5}\right\}\right)\right|=1$ for avoiding a rainbow $2 K_{2} \cup K_{1,3}$, which implies that there is a $k$-connected monochromatic subgraph of order at least $s+t-6$, a contradiction. So $\left|C\left(u_{1}, V \backslash\left\{v_{1}, v_{2}, v_{3}\right\}\right) \cap\{4,5, \ldots, m\}\right| \leq 1$. For any $i \in\{1,2,3\}$, if $\left|C\left(v_{i}, U \backslash\left\{u_{1}\right\}\right) \cap\{4,5, \ldots, m\}\right| \geq 4$, say $c\left(v_{i} u_{j}\right)=j+2$ for every $j \in\{2,3,4,5\}$, then $c\left(u_{6} v_{4}\right) \notin[m]$ for avoiding a rainbow $2 K_{2} \cup K_{1,3}$, a contradiction. Thus $\left|C\left(v_{i}, U \backslash\left\{u_{1}\right\}\right) \cap\{4,5, \ldots, m\}\right| \leq 3$ for each $i \in\{1,2,3\}$.

Since there are at least 16 colors used in $G$, we have $\mid C\left(V(G) \backslash\left\{u_{1}, v_{1}, v_{2}\right.\right.$, $\left.\left.v_{3}\right\}\right) \cap\{4,5, \ldots, m\} \mid \geq 3$. In order to avoid a rainbow $2 K_{2} \cup K_{1,3}$, we may assume that there is a rainbow $K_{1,3}$ using colors $m-2, m-1$ and $m$ in 
$G-\left\{u_{1}, v_{1}, v_{2}, v_{3}\right\}$, say with vertex set $W$. Then for any edge $e \in E(G-W \cup$ $\left.\left\{u_{1}, v_{1}, v_{2}, v_{3}\right\}\right)$, we have $c(e) \notin[m]$, a contradiction.

From Proposition 6.20, Lemmas 6.23 and 6.24, we conclude that the statement of Theorem 6.5 is valid.

\subsection{Large $k$-connected 2-colored subgraphs in every Gallai-3-coloring}

We first state and prove two lemmas that immediately imply Question 6.3 has an affirmative answer for $1 \leq k \leq 3$.

Lemma 6.25. For any integer $n \geq 7$, every Gallai-3-coloring of $K_{n}$ contains a 2-connected 2-colored subgraph of order $n$.

Proof. Let $G$ be a Gallai-3-coloring of $K_{n}$ and suppose for a contradiction that $G$ contains no 2-connected spanning subgraph using at most two colors. We first prove the following claim concerning every 3-edge-coloring of $K_{n}$.

Claim 6.2. For any integer $n \geq 7$, there is a 2-connected 2-colored subgraph of order $n-1$ in every 3-edge-coloring (not necessarily a Gallai-coloring) of $K_{n}$.

Proof. Let $F$ be a 3-edge-coloring of $K_{n}$ using red, blue and green. For a contradiction, suppose that $F$ contains no 2-connected 2-colored subgraph of order $n-1$. By Theorem 6.1, there exists a 2-connected subgraph $R$ of order at least $n-2$ using either \{green\} or \{red, blue\}. If $|V(R)| \geq n-1$, then $R$ is a desired subgraph, so we may assume that $|V(R)|=n-2$.

Let $V(F) \backslash V(R)=\{u, v\}$. First we consider the case that $R$ is a monochromatic subgraph colored by green. Since $|V(R)|=n-2 \geq 5$, there are at least two edges between $u$ and $V(R)$ using a single color. Then $R$ and $u$ form a 2-connected subgraph of order $n-1$ using at most two colors, a contradiction. Therefore, we may further assume that $R$ is colored by red and blue. Then there is at most one edge using red or blue between $u$ (resp., $v$ ) and $R$. Hence, there is a subset $R^{\prime} \subset V(R)$ with $\left|R^{\prime}\right|=n-4 \geq 3$ such that $c\left(\{u, v\}, R^{\prime}\right)$ is 
green. Then there is a monochromatic complete bipartite graph $K_{2, n-4}$ colored by green, which is 2 -connected. Let $V(R) \backslash R^{\prime}=\{x, y\}$. Then we can find a 2-connected 2-colored subgraph with vertex set $R^{\prime} \cup\{u, v, x\}$ by a similar argument to the first case, a contradiction.

By Claim 6.2, $G$ contains a 2-connected 2-colored (say red and blue) subgraph $H$ of order $n-1$. Let $V(G) \backslash V(H)=\{v\}$. Then there is at most one edge using red or blue between $v$ and $H$. First suppose that $c(v u)$ is red and $c(v, V(H) \backslash\{u\})$ is green for some $u \in V(H)$. In order to avoid a rainbow triangle, we have that $C(u, V(H) \backslash\{u\}) \subseteq$ red, green $\}$. Then we obtain a $K_{2} \vee \overline{K_{n-2}}$ using red and green, which is a 2-connected 2-colored subgraph of order $n$. Thus we may further assume that $c(v, V(H))$ is green. Since any 2-edge-coloring of a complete graph contains a monochromatic spanning tree, there is a spanning tree colored by either \{red\} or \{blue, green\} in $G[V(H)]$. In both cases, such a spanning tree together with vertex $v$ forms a 2-connected spanning subgraph of $G$ using at most two colors, a contradiction.

Lemma 6.26. For any integer $n \geq 7$, every Gallai-3-coloring of $K_{n}$ contains a 3-connected 2-colored subgraph of order at least $n-1$.

Proof. Let $G$ be a Gallai-3-coloring of $K_{n}$, say using red, blue and green. Suppose that $G$ contains no 3-connected 2-colored subgraph of order at least $n-1$. By Lemma 6.25, $G$ contains a 2-connected spanning subgraph $H$ using at most two colors, say red and blue. Note that $H$ is not 3-connected; otherwise $H$ would be the desired subgraph. So we may further assume that $X=\left\{x_{1}, x_{2}\right\}$ is a vertex cut of $H$. Note that $H-X$ has at least two components. Let $A$ be the union of vertices in some components of $H-X$ and let $B$ be the union of vertices in the remaining components of $H-X$ such that $|A| \geq|B|>0$. Then all the edges between $A$ and $B$ are colored by green, that is, $G[A \cup B]$ contains a monochromatic complete bipartite graph $H^{\prime}$ of order $n-2$ using green.

If $|B| \geq 3$, then $H^{\prime}$ is 3-connected. Furthermore, since $|A \cup B|=n-2 \geq 5$, there are at least three edges between $x_{1}$ and $A \cup B$ using either \{red\} or \{blue, green $\}$. If the former holds, then $G-\left\{x_{2}\right\}$ contains a 3-connected subgraph using red and green of order $n-1$. And if the latter holds, then $G-\left\{x_{2}\right\}$ 
contains a 3-connected subgraph using blue and green of order $n-1$. In both cases we can derive a contradiction. Thus $1 \leq|B| \leq 2$.

If $|B|=2$, then let $B=\left\{b_{1}, b_{2}\right\}$. There exists at least one edge using red or blue between $B$ and $X$, say $c\left(x_{1} b_{1}\right)$ being red. Then $C\left(x_{1}, A\right) \subseteq\{$ red, green $\}$ in order to avoid a rainbow triangle. Then $G-\left\{x_{2}\right\}$ contains a 3-connected subgraph $K_{3, n-4}$ using red and green, a contradiction.

If $|B|=1$, say $B=\{b\}$, then $|A|=n-3 \geq 4$. If $c\left(x_{1} b\right)$ (resp., $c\left(x_{2} b\right)$ ) is green, then $H-\left\{x_{2}\right\}$ (resp., $H-\left\{x_{1}\right\}$ ) is disconnected, contradicting the fact that $H$ is 2-connected. Thus $C(B, X) \subseteq\{$ red, blue $\}$. Without loss of generality, let $x_{1} b$ be colored by red. Then $C\left(x_{1}, A\right) \subseteq\{$ red, green $\}$ for avoiding a rainbow triangle. Likewise if $x_{2} b$ is colored by red, then $C\left(x_{2}, A\right) \subseteq$ \{red, green $\}$ holds once again. But then $G$ contains a 3-connected subgraph using red and green of order $n$, a contradiction. Thus $x_{2} b$ cannot be colored by red, so $c\left(x_{2} b\right)$ is blue. In order to avoid a rainbow triangle, we also have $C\left(x_{2}, A\right) \subseteq$ \{blue, green\}. Since $G[A]$ is a Gallai-3-coloring of $K_{|A|}$, there exists a color which spans a connected subgraph by Corollary 5.13. If such a color is green or red, then $G-\left\{x_{2}\right\}$ contains a 3-connected subgraph of order $n-1$ using green and red, a contradiction. Thus this color is blue, but then $G-\left\{x_{1}\right\}$ contains a 3-connected subgraph using blue and green of order $n-1$, a contradiction. This completes the proof of Lemma 6.26.

Next, we construct a counterexample to show that Question 6.3 has a negative answer when $k=4 t$.

Proposition 6.27. For any positive integer $t$ and sufficiently large integer $n$, let $k=4 t$. There exists a Gallai-3-coloring of $K_{n}$ which contains no $k$-connected 2-colored subgraph of order at least $n-\lfloor(k-1) / 2\rfloor$.

Proof. Since $k=4 t$, we have $\lfloor(k-1) / 2\rfloor=2 t-1$. Note that the edge set of $K_{4 t}$ can be partitioned into $4 t-1$ perfect matchings. Using $2 t-1$ of them completely and half of an additional one, we obtain a graph $F$ of order $4 t$ with degree sequence $\left(d_{1}, d_{2}, \ldots, d_{4 t}\right)$, where $d_{1}=d_{2}=\cdots=d_{2 t}=2 t$ and $d_{2 t+1}=$ $d_{2 t+2}=\cdots=d_{4 t}=2 t-1$. Let $\left\{v_{1}, v_{2}, \ldots, v_{2 t}\right\}$ and $\left\{v_{2 t+1}, v_{2 t+2}, \ldots, v_{4 t}\right\}$ be the sets of vertices with degree $2 t$ and $2 t-1$ in $F$ (i.e., degree $2 t-1$ and $2 t$ in $\bar{F}$ ), respectively. 
We color all the edges of $F$ using color 1, and add an edge with color 2 between each pair of vertices that are non-adjacent in $F$. Let $H$ denote the resulting edge-colored $K_{4 t}$. Let $G$ be a copy of $K_{n}$ with $V(G)=V_{1} \cup V_{2} \cup V_{3}$, where $\left|V_{1}\right|=n-6 t,\left|V_{2}\right|=4 t$ and $\left|V_{3}\right|=2 t$. We color the edges of $G$ such that $G\left[V_{2}\right]=H, c\left(\left\{v_{1}, v_{2}, \ldots, v_{2 t}\right\}, V_{1}\right)=1, c\left(\left\{v_{2 t+1}, v_{2 t+2}, \ldots, v_{4 t}\right\}, V_{1}\right)=2$, and all the remaining edges are colored by color 3 (see Figure 6.4 for an illustration of the case $t=1$ ).

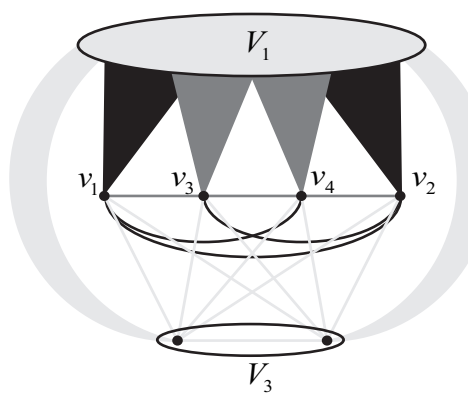

Figure 6.4: A Gallai-3-coloring of $K_{n}$ without 4-connected 2colored subgraphs of order $n-1$.

We first show that $G$ is a Gallai-coloring. Suppose that there is a rainbow triangle with vertex set $W$. Note that for any vertex $v \in V_{3}$, we have $c(v, V(G) \backslash$ $\{v\})=3$. Thus $W \cap V_{3}=\emptyset$. Since $\left|C\left(V_{1}\right)\right|=1$ and $\left|C\left(V_{2}\right)\right|=2$, we have $\left|W \cap V_{1}\right| \leq 2$ and $\left|W \cap V_{2}\right| \leq 2$. Moreover, since $\left|C\left(V_{2} \cup\{u\}\right)\right|=2$ for any vertex $u \in V_{1}$, we have $\left|W \cap V_{1}\right|=2$ and $\left|W \cap V_{2}\right|=1$. On the other hand, since for any $v \in V_{2},\left|C\left(v, V_{1}\right)\right|=1$, we also have $\left|W \cap V_{2}\right|=2$ and $\left|W \cap V_{1}\right|=1$, a contradiction. Thus $G$ is a Gallai-coloring.

Suppose that $G$ contains a $k$-connected 2-colored subgraph $S$ such that $|V(S)| \geq n-\lfloor(k-1) / 2\rfloor=n-2 t+1$. Since $c\left(V_{3}, V_{1} \cup V_{2}\right)=3$ and $\left|V_{3}\right|=2 t$, we have $3 \in C(S)$. If $C(S)=\{1,3\}$, then $v_{2 t+1}, v_{2 t+2}, \ldots, v_{4 t} \notin V(S)$ since there are exactly $4 t-1$ edges using color 1 or color 3 between $x$ and $V(G) \backslash\{x\}$ for every $x \in\left\{v_{2 t+1}, v_{2 t+2}, \ldots, v_{4 t}\right\}$. But then $|V(S)| \leq n-2 t<n-2 t+1$, a contradiction. If $C(S)=\{2,3\}$, then we can also derive a contradiction in a similar way. Thus $G$ contains no $k$-connected 2-colored subgraph of order at least $n-\lfloor(k-1) / 2\rfloor$. 
Although Question 6.3 has a negative answer when $k=4 t$, we still believe that the following conjecture is true.

Conjecture 6.2. For any integer $k \geq 1$ and sufficiently large integer $n$, if $k \neq 4 t$ for any positive integer $t$, then every Gallai-3-coloring of $K_{n}$ contains a $k$-connected 2-colored subgraph of order at least $n-\lfloor(k-1) / 2\rfloor$, and if $k=4 t$ for some positive integer $t$, then every Gallai-3-coloring of $K_{n}$ contains a $k$-connected 2-colored subgraph of order at least $n-k / 2$.

From the above argument, Conjecture 6.2 is true in the special case $1 \leq k \leq 3$. We can also verify this conjecture in the case $k=4$.

Proposition 6.28. For any integer $n \geq 11$, every Gallai-3-coloring of $K_{n}$ contains a 4-connected 2-colored subgraph of order at least $n-2$.

Proof. Let $G$ be a Gallai-3-coloring of $K_{n}$, say using red, blue and green. Suppose that $G$ contains no 4-connected 2-colored subgraph of order at least $n-2$. By Lemma 6.26, $G$ contains a 3-connected 2-colored (say red and blue) subgraph $H$ of order at least $n-1$. Note that $H$ is not 4-connected; otherwise $H$ would be the desired subgraph. So we may further assume that $X=\left\{x_{1}, x_{2}, x_{3}\right\}$ is a vertex cut of $H$. Note that $H-X$ has at least two components. Let $A$ be the union of vertices in some components of $H-X$ and let $B$ be the union of vertices in the remaining components of $H-X$ such that $|A| \geq|B|>0$. Then all the edges between $A$ and $B$ are colored by green.

For every $i \in[3]$, if $\{$ red $\} \subseteq C\left(x_{i}, B\right)$ (resp., \{blue $\subseteq C\left(x_{i}, B\right)$ ), then $C\left(x_{i}, A\right) \subseteq$ red, green $\}$ (resp., $C\left(x_{i}, A\right) \subseteq\{$ blue, green $\}$ ) for avoiding a rainbow triangle. Moreover, $C\left(x_{i}, A\right) \cap\left\{\right.$ red, blue $\neq \neq \emptyset$ and $C\left(x_{i}, B\right) \cap$ red, blue $\} \neq \emptyset$; otherwise $H$ is not 3-connected. By the Pigeonhole Principle, we may assume that $\{$ red $\} \subseteq C\left(x_{1}, B\right)$ and $\{$ red $\} \subseteq C\left(x_{2}, B\right)$ without loss of generality. Thus $C\left(x_{1}, A\right) \subseteq\{$ red, green $\}$ and $C\left(x_{2}, A\right) \subseteq\{$ red, green $\}$.

Note that $|A| \geq\lceil(n-4) / 2\rceil \geq 4$. If $|B| \geq 2$, then $\left|B \cup\left\{x_{1}, x_{2}\right\}\right| \geq 4$. Thus $G\left[A \cup B \cup\left\{x_{1}, x_{2}\right\}\right]$ contains a 4-connected 2-colored (using red and green) subgraph $K_{|A|,|B|+2}$, a contradiction. Hence, $|B|=1$. Let $B=\{b\}$. Now $c\left(\left\{x_{1}, x_{2}\right\}, b\right)$ is red and $C\left(\left\{x_{1}, x_{2}\right\}, A\right) \subseteq\{$ red, green $\}$. We claim that $c\left(x_{3} b\right)$ is blue. Indeed, if $c\left(x_{3} b\right)$ is red, then $C\left(x_{3}, A\right) \subseteq$ red, green $\}$. This implies 
that $G$ contains a 4-connected 2-colored (using red and green) subgraph $K_{4,|A|}$, a contradiction. Thus $c\left(x_{3} b\right)$ is blue, so $C\left(x_{3}, A\right) \subseteq\{$ blue, green $\}$.

By Corollary 5.13, $G[A]$ contains a connected monochromatic spanning subgraph $F$. If $C(F)$ is red or green, then $G\left[A \cup B \cup\left\{x_{1}, x_{2}\right\}\right]$ contains a 4-connected 2-colored (using red and green) subgraph of order at least $n-2$, a contradiction. Thus $C(F)$ is blue. Recall that $\{$ red $\} \subseteq C\left(\left\{x_{1}, x_{2}\right\}, A\right) \subseteq$ red, green $\}$. In order to avoid a rainbow triangle, we have that $c\left(\left\{x_{1}, x_{2}\right\}, A\right)$ is red.

We first suppose $|V(H)|=n-1$. Let $\{v\}=V(G) \backslash V(H)$. Then $\mid C(v, A) \cap$ \{red, green $\} \mid \leq 1$ for avoiding a rainbow triangle. Thus $C(v, A) \subseteq$ red, blue $\}$ or $C(v, A) \subseteq\{$ blue, green $\}$. In the former case, $G-\left\{x_{3}, b\right\}$ contains a 4-connected 2-colored (using red and blue) subgraph of order $n-2$, a contradiction. In the latter case, $G-\left\{x_{1}, x_{2}\right\}$ contains a 4-connected 2-colored (using blue and green) subgraph of order $n-2$, a contradiction.

Thus $|V(H)|=n$. Note that $|A|=n-4 \geq 7$. By Lemma 6.25, $G[A]$ contains a 2-connected 2-colored spanning subgraph $F^{\prime}$. If $F^{\prime}$ is colored by red and blue (resp., red and green), then $G\left[A \cup\left\{x_{1}, x_{2}\right\}\right]$ contains a 4-connected 2-colored subgraph of order $n-2$ using red and blue (resp., red and green), a contradiction. If $F^{\prime}$ is colored by blue and green, then $G\left[A \cup\left\{x_{3}, b\right\}\right]$ contains a 4-connected 2-colored subgraph of order $n-2$ using blue and green, a contradiction. This completes the proof of Proposition 6.28.

\subsection{Concluding remarks}

In this chapter, we mainly focussed on forcing large $k$-connected monochromatic subgraphs. Instead of forcing a $k$-connected monochromatic subgraph, it is interesting to consider conditions that guarantee a long monochromatic path or cycle in an edge-colored complete graph. Using a result of Erdős and Gallai [60] that every graph $G$ on $n$ vertices with average degree greater than $k-2$ contains a path $P_{k}$ in $G$, we can prove the following result.

Proposition 6.29. Let $G$ be an m-edge-coloring of $K_{n}$. For any non-negative integers $a_{1}, a_{2}, \ldots, a_{m}$ with $\sum_{i=1}^{m} a_{i} \leq n+2 m-2, G$ contains a monochromatic copy of $P_{a_{i}}$ in color $i$ for some $i \in[m]$. 
Proof. If $\min _{1 \leq i \leq m}\left\{a_{1}, a_{2}, \ldots, a_{m}\right\} \leq 2$, then $G$ contains a monochromatic $P_{2}$ clearly. Thus we may assume that $\min _{1 \leq i \leq m}\left\{a_{1}, a_{2}, \ldots, a_{m}\right\} \geq 3$. For $v \in V(G)$ and $i \in[m]$, let $d_{i}(v)$ denote the number of edges incident with $v$ using color $i$. Then for any $v \in V(G)$, we have $\sum_{i=1}^{m} d_{i}(v)=n-1 \geq \sum_{i=1}^{m} a_{i}-2 m+1$. Let $\bar{d}_{i}(G)=\frac{1}{n} \sum_{v \in V(G)} d_{i}(v)$ for $1 \leq i \leq m$. Then

$$
\begin{aligned}
\sum_{i=1}^{m} \bar{d}_{i}(G) & =\sum_{i=1}^{m}\left(\frac{1}{n} \sum_{v \in V(G)} d_{i}(v)\right)=\frac{1}{n} \sum_{v \in V(G)}\left(\sum_{i=1}^{m} d_{i}(v)\right) \\
& \geq \frac{1}{n} \sum_{v \in V(G)}\left(\sum_{i=1}^{m} a_{i}-2 m+1\right)=\sum_{i=1}^{m} a_{i}-2 m+1,
\end{aligned}
$$

and thus there exists an $i \in[m]$ such that $\bar{d}_{i}(G)>a_{i}-2$. By Erdős and Gallai's result mentioned above, $G$ contains a monochromatic copy of $P_{a_{i}}$ in color $i$.

By setting $a_{1}=a_{2}=\cdots=a_{m}=\left\lfloor\frac{n+2 m-2}{m}\right\rfloor$ in Proposition 6.29, there is a monochromatic copy of $P_{t}$ with $t \geq\left\lfloor\frac{n+2 m-2}{m}\right\rfloor=\left\lfloor\frac{n-2}{m}\right\rfloor+2$ in any $m$ edge-coloring of $K_{n}$. For monochromatic cycles, Kano and Li [104] showed that there is a monochromatic cycle of length at least $\left\lceil\frac{n}{m}\right\rceil$ in any $m$-edgecoloring of $K_{n}$. It is natural to consider the problem which forbidden rainbow subgraphs force long monochromatic paths or cycles in edge-colorings of $K_{n}$. 


\section{Chapter 7}

\section{Integer colorings with no rainbow 3-term arithmetic progression}

In this chapter, we study the rainbow Erdös-Rothschild problem with respect to 3-term arithmetic progressions. We obtain the asymptotic number of $r$ colorings of $[n]$ without rainbow 3-term arithmetic progressions, and we show that the typical colorings with this property are 2-colorings. We also prove that $[n]$ attains the maximum number of rainbow 3-term arithmetic progression-free $r$-colorings among all subsets of $[n]$. Moreover, the exact number of rainbow 3-term arithmetic progression-free $r$-colorings of $\mathbb{Z}_{p}$ is obtained, where $p$ is any prime and $\mathbb{Z}_{p}$ is the cyclic group of order $p$.

\subsection{Introduction}

Two fundamental topics in extremal combinatorics are counting the number of discrete structures that have certain properties and analyzing the typical properties of those structures. One of the major problems in this area, initiated by Erdős, Kleitman and Rothschild [65], is to determine the number of $\mathrm{H}$ free graphs on $n$ vertices and characterize their typical structure. We refer 
to $[11,16,115,143]$ and the references therein for related results.

In the setting of integers, analogous problems were raised by Cameron and Erdős [32], who studied the number of subsets of positive integers satisfying certain constraints. We refer to $[13,14,129]$ for results on the number of sum-free subsets, to [12] for results on the number of subsets with no $k$-term arithmetic progression, and to [126] for results on multiplicative Sidon sets.

In the context of colored discrete structures, Erdős and Rothschild [57] asked which graph on $n$ vertices admits the maximum number of $r$-edgecolorings without a monochromatic subgraph $H$. This Erdős-Rothschild problem was extended to edge-colorings of graphs with other forbidden coloring patterns. For example, the number of rainbow triangle-free $r$-edge-colorings of complete graphs and their typical structure were determined in $[10,17]$. This problem can also be generalized to other discrete structures. Hoppen, Kohayakawa and Lefmann [99] studied the Erdős-Rothschild extension of the celebrated Erdős-Ko-Rado Theorem on hypergraphs. Liu, Sharifzadeh and Staden [130] and Hàn and Jiménez [94] determined the maximum number of monochromatic sum-free colorings in integers and in finite abelian groups, respectively. Very recently, motivated by these results, Cheng et al. [35] studied the number of rainbow sum-free colorings of integers and their typical structure.

In this chapter, we focus on colorings of $[n]$ without rainbow $k$-term arithmetic progressions. Before we continue, we recall some of the crucial definitions.

Given a subset $A \subseteq[n]$, a $k$-term arithmetic progression ( $k$-AP) of $A$ is a sequence in $A$ of the form $a, a+d, a+2 d, \ldots, a+(k-1) d$, where $d \in[n]$. For an integer $r \geq 1$ and a subset $A \subseteq[n]$, let $c: A \rightarrow[r]$ be an $r$-coloring of $A$. Given an $r$-coloring $c$ of $A$, a $k$-AP of $A$ is called rainbow if $c(a+i d) \neq c(a+j d)$ for every $0 \leq i<j \leq k-1$. An $r$-coloring of $A$ is called rainbow $k$-AP-free if it does not contain a rainbow $k$-AP.

In 1927, Van der Waerden [169] proved that for any positive integers $k$ and $r$, if $n$ is sufficiently large, then every $r$-coloring of $[n]$ contains a monochromatic $k$-AP; see Theorem 1.2. A strengthening was conjectured by Erdős and Turán [69], and proved by Szemerédi [167] in 1975. 
Theorem 7.1 (Szemerédi's Theorem [167]). Let $k$ be a positive integer and let $\delta>0$. Then there exists a positive integer $s z(k, \delta)$ such that for all $n \geq$ $s z(k, \delta)$, every subset $C \subseteq[n]$ with $|C|>\delta$ contains a $k-A P$.

In [61], Erdős and Graham proved a canonical version of Van der Waerden's Theorem, that is, for any integer $k \geq 3$, if $n$ is sufficiently large, then every coloring (with any number of colors) of $[n]$ contains either a rainbow $k$-AP or a monochromatic $k$-AP. For more results related to rainbow arithmetic progressions, see $[4,19,29,103,131,150]$.

For any $A \subseteq[n]$ and $r \geq 3$, we use $g_{r}\left(A,[n]\right.$ ) (or simply $g_{r}(A)$ ) to denote the number of rainbow 3-AP-free $r$-colorings of $A$. By choosing two of the $r$ colors and coloring the elements of $A$ arbitrarily with these two colors, a lower bound on $g_{r}(A)$ is

$$
g_{r}(A) \geq\left(\begin{array}{l}
r \\
2
\end{array}\right)\left(2^{|A|}-2\right)+r=\left(\begin{array}{l}
r \\
2
\end{array}\right) 2^{|A|}-r^{2}+2 r .
$$

For the upper bound, we shall prove the following result in Section 7.3.

Theorem 7.2. For all integers $r \geq 3$ and any real number $\xi$ with $0<\xi \leq$ $\frac{3}{5+8 \log _{2} r}$, there exists $n_{0} \in \mathbb{N}$ such that for all $n \geq n_{0}$ the following holds. If $A \subseteq[n]$ and $|A| \geq(1-\xi) n$, then the number of rainbow 3-AP-free r-colorings of $A$ is at most

$$
\left(\begin{array}{l}
r \\
2
\end{array}\right) 2^{|A|}+2^{-\frac{n}{36 \log _{2} n}} 2^{n}
$$

In view of inequality (7.1), the upper bound given by Theorem 7.2 is asymptotically tight for $A=[n]$, and the description of the typical structure follows immediately.

Corollary 7.3. For every integer $r \geq 3$, there exists $n_{0} \in \mathbb{N}$ such that for all $n \geq n_{0}$ we have $g_{r}([n])=\left(\left(\begin{array}{l}r \\ 2\end{array}\right)+o(1)\right) 2^{n}$. Moreover, almost all rainbow 3-AP-free $r$-colorings of $[n]$ are 2-colorings.

Next, we answer the question which subset(s) of $[n]$ admit the maximum number of rainbow 3-AP-free $r$-colorings, among all subsets of $[n]$. Using Szemerédi's Theorem and Theorem 7.2, we shall prove the following result in Section 7.3. 
Theorem 7.4. For all integers $r \geq 3$, there exists $n_{1} \in \mathbb{N}$ such that for all $n \geq n_{1}$ and all subset $A \subset[n]$, we have $g_{r}(A)<g_{r}([n])$. Thus $[n]$ is the unique subset admitting the maximum number of rainbow 3-AP-free $r$-colorings among all subsets of $[n]$.

Let $\mathbb{Z}_{n}$ be the cyclic group of order $n$ formed by the set $\{0,1, \ldots, n-1\}$ with the operation of addition modulo $n$. A 3 -AP in $\mathbb{Z}_{n}$ is a sequence $a, b, c$ such that $a+c \equiv 2 b(\bmod n)$. For any $A \subseteq \mathbb{Z}_{n}$ and $r \geq 3$, we use $g_{r}\left(A, \mathbb{Z}_{n}\right)$ to denote the number of rainbow 3-AP-free $r$-colorings of $A$, and we use the shorthand $g_{r}\left(\mathbb{Z}_{n}\right)$ for $g_{r}\left(\mathbb{Z}_{n}, \mathbb{Z}_{n}\right)$. For any $A \subseteq[n]$, if we also view $A$ as a subset of $\mathbb{Z}_{n}$, then $g_{r}\left(A, \mathbb{Z}_{n}\right) \leq g_{r}(A,[n])$ clearly. Moreover, the lower bound $g_{r}\left(A, \mathbb{Z}_{n}\right) \geq\left(\begin{array}{c}r \\ 2\end{array}\right) 2^{|A|}-r^{2}+2 r$ also holds. Furthermore, it follows from the proof of Theorem 7.4 (see Section 7.3) that $g_{r}(A,[n])<\left(\begin{array}{l}r \\ 2\end{array}\right) 2^{n}-r^{2}+2 r$ for every $A \subset[n]$. Hence, we have the following corollary.

Corollary 7.5. For every integer $r \geq 3$, there exists $n_{0} \in \mathbb{N}$ such that for all $n \geq n_{0}$ we have

$$
\left(\begin{array}{l}
r \\
2
\end{array}\right) 2^{n}-r^{2}+2 r \leq g_{r}\left(\mathbb{Z}_{n}\right) \leq\left(\begin{array}{l}
r \\
2
\end{array}\right) 2^{n}+2^{-\frac{n}{36 \log _{2} n}} 2^{n},
$$

and thus almost all rainbow 3-AP-free r-colorings of $\mathbb{Z}_{n}$ are 2-colorings. Moreover, we have $g_{r}\left(A, \mathbb{Z}_{n}\right)<g_{r}\left(\mathbb{Z}_{n}\right)$ for any $A \subset \mathbb{Z}_{n}$.

Given two positive integers $n$ and $k$, the anti-van der Waerden number $a w([n], k)$ (resp., $a w\left(\mathbb{Z}_{n}, k\right)$ ) is the smallest $r$ such that every exact $r$-coloring of $[n]$ (resp., $\mathbb{Z}_{n}$ ) contains a rainbow $k$-AP, where an exact $r$-coloring is a coloring using all the $r$ colors. For an integer $n$, if $a w\left(\mathbb{Z}_{n}, 3\right)=3$, then all the rainbow 3-AP-free $r$-colorings of $\mathbb{Z}_{n}$ are 2-colorings, so $g_{r}\left(\mathbb{Z}_{n}\right)=\left(\begin{array}{l}r \\ 2\end{array}\right) 2^{n}-$ $r^{2}+2 r$. In [103], Jungić et al. gave a characterization of integers $n$ such that $a w\left(\mathbb{Z}_{n}, 3\right)=3$. Moreover, Butler et al. [29] proved that $3 \leq a w\left(\mathbb{Z}_{p}, 3\right) \leq 4$ for any prime $p$. Let $\mathbb{Z}_{n}^{\times}$be the multiplicative group of integers modulo $n$, and let $\operatorname{ord}_{n}(2)$ be the order of 2 in $\mathbb{Z}_{n}^{\times}$. In Section 7.4, we shall determine the exact value of $g_{r}\left(\mathbb{Z}_{p}\right)$ for any odd prime. 
Theorem 7.6. For any prime $p \geq 3$ and integer $r \geq 3$, we have

$$
g_{r}\left(\mathbb{Z}_{p}\right)=\left(\begin{array}{l}
r \\
2
\end{array}\right) 2^{p}-r^{2}+2 r+r\left(\begin{array}{c}
r-1 \\
2
\end{array}\right) p\left(2^{\frac{p-1}{c \cdot \text { ord } p(2)}}-2\right),
$$

where $c=1$ if $\operatorname{ord}_{p}(2)$ is even, and $c=2$ otherwise.

\subsection{Additional notation and preliminaries}

We will prove Theorem 7.2 using the Hypergraph Container Method developed by Balogh-Morris-Samotij [15] and Saxton-Thomason [160] independently. We first introduce some additional notation.

Let $\mathscr{H}$ be a $k$-uniform hypergraph. Let $d(\mathscr{H})$ be the average degree of $\mathscr{H}$. For a subset $U \subseteq V(\mathscr{H})$, let $\mathscr{H}[U]$ be the subhypergraph of $\mathscr{H}$ induced by $U$, and let $d(U):=|\{e \in E(\mathscr{H}): U \subseteq e\}|$ be the co-degree of $U$. For $2 \leq j \leq k$, the jth maximum co-degree of $\mathscr{H}$ is $\Delta_{j}(\mathscr{H}):=\max \{d(U): U \subseteq V(\mathscr{H}),|U|=j\}$. When the underlying hypergraph is clear, we simply write $d(\mathscr{H})$ and $\Delta_{j}(\mathscr{H})$ as $d$ and $\Delta_{j}$, respectively. For $0<\tau<1$, the co-degree function is defined to be

$$
\Delta(\mathscr{H}, \tau):=2^{\left(\begin{array}{c}
k \\
2
\end{array}\right)-1} \sum_{j=2}^{k} 2^{-\left(\begin{array}{c}
j-1 \\
2
\end{array}\right)} \frac{\Delta_{j}}{d \tau^{j-1}} .
$$

We will use the following form of hypergraph container theorem.

Theorem 7.7 (Hypergraph container theorem, see $[10,35,160]$ ). Let $\mathscr{H}$ be a $k$-uniform hypergraph on vertex set $[N]$. Let $0<\varepsilon, \tau<1 / 2$. Suppose that $\tau<1 /\left(200 k !^{2} k\right)$ and $\Delta(\mathscr{H}, \tau) \leq \varepsilon /(12 k !)$. Then there exists $c=c(k) \leq$ $1000 k !^{3} k$ and a collection $\mathscr{C}$ of vertex subsets such that

(1) every independent set in $\mathscr{H}$ is a subset of some $C \in \mathscr{C}$;

(2) for every $C \in \mathscr{C},|E(\mathscr{H}[C])| \leq \varepsilon \cdot|E(\mathscr{H})|$;

(3) $\log _{2}|\mathscr{C}| \leq c N \tau \log _{2}(1 / \varepsilon) \log _{2}(1 / \tau)$.

A key concept in applying the hypergraph container method to colored structures is the template, which was first introduced in [71]. 
Definition 7.1. (Template, palette, subtemplate, rainbow 3-AP)

(1) An $r$-template of order $n$ is a function $P:[n] \rightarrow 2^{[r]}$, associating with each integer $x$ in $[n]$ a list of colors $P(x) \subseteq[r]$. We refer to this set $P(x)$ as the palette available at $x$.

(2) Let $P_{1}, P_{2}$ be two $r$-templates of order $n$. We say that $P_{1}$ is a subtemplate of $P_{2}$ (written as $P_{1} \subseteq P_{2}$ ) if $P_{1}(x) \subseteq P_{2}(x)$ for every integer $x \in[n]$.

(3) For an $r$-template $P$ of order $n$, we say that $P$ is a rainbow 3-AP if there exist three integers $a, b, c$ in $[n]$ with $a+c=2 b$ such that $|P(a)|=$ $|P(b)|=|P(c)|=1,|P(x)|=0$ for each $x \in[n] \backslash\{a, b, c\}$, and $P(a)$, $P(b), P(c)$ are pairwise distinct.

(4) For an $r$-template $P$, we say that $P$ is rainbow 3 -AP-free if there is no subtemplate that is a rainbow 3-AP.

Note that for any $A \subseteq[n]$, an $r$-coloring of $A$ can be viewed as an $r$ template $P$ with $|P(x)|=1$ for each $x \in A$ and $|P(x)|=0$ for each $x \in[n] \backslash A$. For an $r$-template $P$, let $R(P)$ be the number of subtemplates of $P$ that are rainbow 3-APs. We should remark that we only consider nontrivial 3-APs, that is, we do not consider the 3-APs with common difference 0 . Let $f(n)$ be the number of 3-APs in $[n]$. Note that there is a bijection between all the 3-APs and ordered pairs $(a, b) \in[n]^{2}$ with $a<b$ and $a \equiv b(\bmod 2)$. Thus $f(n)=\left(\begin{array}{c}{[n / 2\rceil} \\ 2\end{array}\right)+\left(\begin{array}{c}\lfloor n / 2\rfloor \\ 2\end{array}\right)=\left\lfloor\frac{n}{2}\right\rfloor \cdot\left\lfloor\frac{n-1}{2}\right\rfloor$. Throughout this chapter, we will assume that $n$ is large enough. Using Theorem 7.7, we can prove the following container theorem for rainbow 3-AP-free colorings.

Theorem 7.8. For every integer $r \geq 3$, there exists $c=c(r)$ and a collection $\mathscr{C}$ of $r$-templates of order $n$ such that

(1) every rainbow 3-AP-free r-template of order $n$ is a subtemplate of some $P \in \mathscr{C}$;

(2) for every $P \in \mathscr{C}, R(P) \leq n^{-1 / 3} f(n)$;

(3) $\log _{2}|\mathscr{C}| \leq c n^{2 / 3} \log _{2}^{2} n$. 
Proof. Let $\mathscr{H}$ be a 3-uniform hypergraph with vertex set $[n] \times[r]$, whose edges are all triples $\{(a, i),(b, j),(c, \ell)\}$ such that $a, b, c$ form a 3 -AP in $[n]$ and $i, j, \ell$ are three pairwise distinct colors in $[r]$. In other words, every hyperedge in $\mathscr{H}$ corresponds to a rainbow 3 -AP in $[n]$. Note that every vertex subset of $\mathscr{H}$ corresponds to an $r$-template of order $n$, and every independent set in $\mathscr{H}$ corresponds to a rainbow 3-AP-free $r$-template of order $n$. Hence, it suffices to show that for appropriate $\varepsilon$ and $\tau$, there exists a collection $\mathscr{C}$ of vertex subsets satisfying Theorem 7.7 (1)-(3). To achieve this, we need to check that $\Delta(\mathscr{H}, \tau) \leq \varepsilon /(12 \cdot 3 !)$.

Since there are exactly $r(r-1)(r-2)$ ways to rainbow color a 3-AP with $r$ colors, the average degree $d$ of $\mathscr{H}$ satisfies

$$
d=\frac{3|E(\mathscr{H})|}{|V(\mathscr{H})|}=\frac{3 r(r-1)(r-2) f(n)}{n r} \geq \frac{3(r-1)(r-2) n}{5} .
$$

Note that we have $\Delta_{2}=3(r-2)$ and $\Delta_{3}=1$. Let $\varepsilon=n^{-1 / 3} /(r(r-1)(r-2))$ and $\tau=(48 \cdot 3 ! \cdot r)^{1 / 2} n^{-1 / 3}$. Then

$$
\begin{aligned}
\Delta(\mathscr{H}, \tau) & =\frac{4 \Delta_{2}}{d \tau}+\frac{2 \Delta_{3}}{d \tau^{2}} \leq \frac{20}{(r-1) n \tau}+\frac{10}{3(r-1)(r-2) n \tau^{2}} \\
& \leq \frac{11}{3(r-1)(r-2) n \tau^{2}} \leq \frac{\varepsilon}{12 \cdot 3 !} .
\end{aligned}
$$

The result follows.

Definition 7.2. (Good $r$-template) For any $A \subseteq[n]$, an $r$-template $P$ of order $n$ is a good $r$-template of $A$ if it satisfies the following properties:

(1) $|P(x)| \geq 1$ for every $x \in A$;

(2) $R(P) \leq n^{-1 / 3} f(n)$.

For a collection $\mathscr{P}$ of $r$-templates of order $n$ and $A \subseteq[n]$, we use $G(\mathscr{P}, A)$ to denote the set of rainbow 3-AP-free $r$-colorings of $A$ that is a subtemplate of some $P \in \mathscr{P}$. Let $g(\mathscr{P}, A)=|G(\mathscr{P}, A)|$. If $\mathscr{P}=\{P\}$, then we simply write $G(\mathscr{P}, A)$ and $g(\mathscr{P}, A)$ as $G(P, A)$ and $g(P, A)$, respectively. 


\subsection{Colorings of integer sets with no rainbow 3-AP}

We first state and prove some propositions and lemmas.

Proposition 7.9. Let $I \subseteq A \subseteq[n]$. There are at most $\frac{|I|^{2}}{4}+\frac{|I|(n-|A|)}{2}$ ordered pairs $(a, b) \in I^{2}$ with $a<b$ such that $\{a, b\}$ is not contained in any 3-AP of $A$.

Proof. For any ordered pair $(a, b) \in I^{2}$ with $(a+b) / 2 \in A$, we have that $\{a,(a+b) / 2, b\}$ forms a 3 -AP of $A$. Thus if $\{a, b\}$ is not contained in any 3-AP of $A$, then either $a$ and $b$ have different parities, or $(a+b) / 2 \in[n] \backslash A$. Let $\alpha$ be the number of odd integers in $I$. Then the number of ordered pairs $(a, b) \in I^{2}$ with $a<b$ such that $a$ and $b$ have different parities is at most $\alpha(|I|-\alpha) \leq|I|^{2} / 4$. For any $i \in[n] \backslash A$, the number of ordered pairs $(a, b) \in I^{2}$ with $a<b$ and $(a+b) / 2=i$ is at $\operatorname{most} \min \{|[i-1] \cap I|,|([n] \backslash[i]) \cap I|\}$. Thus the number of ordered pairs $(a, b) \in I^{2}$ with $a<b$ and $(a+b) / 2 \in[n] \backslash A$ is at most $|I|(n-|A|) / 2$. The result follows.

Proposition 7.10. Let $I \subseteq A \subseteq[n]$ with $|I| \geq \frac{73}{74} n$, and let $I_{1}, I_{2}$ be a bipartition of $I$ with $\left|I_{1}\right| \leq\left|I_{2}\right|$. There are at least $\frac{\left|I_{1}\right|\left|I_{2}\right|}{9}-3\left|I_{1}\right|(n-|A|)$ pairs $(a, b)$ with $a \in I_{1}$ and $b \in I_{2}$ such that $\{a, b\}$ is contained in some 3-AP of $A$.

Proof. Since $\left|I_{1}\right| \leq\left|I_{2}\right|$, we have $\left|I_{1}\right| \leq|I| / 2 \leq n / 2$ and $\left|I_{2}\right| \geq|I| / 2 \geq$ $73 n / 148$. We first consider the pairs $(a, b)$ with $a \in I_{1}$ and $b \in I_{2}$ such that $\{a, b\}$ is contained in some 3-AP of $[n]$. For any pair $(i, j)$ with $1 \leq i<j \leq n$, if $\{i, j\}$ is not contained in any 3-AP of $[n]$, then $2 i-j \leq 0,2 j-i \geq n+1$, and $i, j$ have different parities. So $i \leq j / 2 \leq n / 2, j \geq 2 i$ and $j \geq(n+1+i) / 2$.

For each $i \in[n]$, the number of integers $j \in[n]$ with $j>i$ such that $\{i, j\}$ is not contained in any 3-AP of $[n]$ is at most $\left\lceil\frac{1}{2}(n-\max \{\min \{2 i-\right.$ $\left.\left.\left.1, n\}, \frac{n+1+i}{2}-1\right\}\right)\right] \leq \alpha(i)$, where $\alpha(i):=\frac{1}{2}\left(n-\max \left\{2 i-1, \frac{n-1+i}{2}\right\}+1\right)$ if $1 \leq i \leq\lfloor n / 2\rfloor$, and $\alpha(i):=0$ if $\lfloor n / 2\rfloor+1 \leq i \leq n$. Note that $\alpha(1) \geq \alpha(2) \geq$ $\cdots \geq \alpha(\lfloor n / 2\rfloor) \geq \alpha(\lfloor n / 2\rfloor+1)=\cdots=\alpha(n)=0$. Similarly, for each $j \in[n]$, the number of integers $i \in[n]$ with $i<j$ such that $\{i, j\}$ is not contained in any 3-AP of $[n]$ is at most $\alpha(n+1-j)$. Let $\beta(j):=\alpha(n+1-j)$ for each $j \in[n]$. Note that $\beta(n) \geq \beta(n-1) \geq \cdots \geq \beta(\lceil n / 2\rceil+1) \geq \beta(\lceil n / 2\rceil)=\cdots=\beta(1)=0$. 
Thus the number pairs of $(a, b)$ with $a \in I_{1}$ and $b \in I_{2}$ such that $\{a, b\}$ is contained in some 3-AP of $[n]$ is at least

$$
\begin{aligned}
& \left|I_{1}\right|\left|I_{2}\right|-\sum_{a \in I_{1}}(\alpha(a)+\beta(a))=\left|I_{1}\right|\left|I_{2}\right|-\sum_{a \in I_{1}} \max \{\alpha(a), \beta(a)\} \\
\geq & \left|I_{1}\right|\left|I_{2}\right|-\left(\alpha(1)+\alpha(2)+\cdots+\alpha\left(\left\lceil\left|I_{1}\right| / 2\right\rceil\right)\right. \\
& \left.+\beta(n)+\beta(n-1)+\cdots+\beta\left(n+1-\left\lfloor\left|I_{1}\right| / 2\right\rfloor\right)\right) \\
\geq & \left|I_{1}\right|\left|I_{2}\right|-2 \sum_{i=1}^{\left\lceil\left|I_{1}\right| / 2\right\rceil} \alpha(i)=\left|I_{1}\right|\left|I_{2}\right|-2 \sum_{i=1}^{\left\lceil\left|I_{1}\right| / 2\right\rceil} \frac{1}{2}\left(n-\frac{n-1+i}{2}+1\right) \\
\geq & \left|I_{1}\right|\left|I_{2}\right|-\frac{\left|I_{1}\right|+1}{2} \cdot \frac{1}{2} \cdot\left(\frac{n+2}{2}+\frac{n-\left|I_{1}\right| / 2+3}{2}\right) \\
= & \left|I_{1}\right|\left|I_{2}\right|-\frac{\left(\left|I_{1}\right|+1\right)\left(2 n-\left|I_{1}\right| / 2+5\right)}{8} \\
\geq & \frac{\left|I_{1}\right|}{4}\left(4\left|I_{2}\right|-2 n+\frac{\left|I_{1}\right|}{2}-5\right) \\
= & \frac{\left|I_{1}\right|}{4}\left(\frac{4}{9}\left|I_{2}\right|+\frac{37}{18}\left|I_{2}\right|+\frac{3}{2}\left|I_{2}\right|+\frac{\left|I_{1}\right|}{2}-2 n-5\right) \\
\geq & \frac{\left|I_{1}\right|}{4}\left(\frac{4}{9}\left|I_{2}\right|+\frac{37}{36}|I|+|I|-2 n-5\right) \\
\geq & \frac{\left|I_{1}\right|}{4}\left(\frac{4}{9}\left|I_{2}\right|+\frac{73}{36} \cdot \frac{73}{74} n-2 n-5\right) \geq \frac{\left|I_{1}\right|\left|I_{2}\right|}{9} .
\end{aligned}
$$

Next, we consider the pairs $(a, b)$ with $a \in I_{1}$ and $b \in I_{2}$ such that $\{a, b\}$ is contained in some 3-AP of $[n]$ but $\{a, b\}$ is not contained in any 3-AP of $A$, and we use $\gamma$ to denote the number of such pairs. In this case, at least one of the following holds: (i) $2 a-b \in[n] \backslash A$, (ii) $(a+b) / 2 \in[n] \backslash A$, (iii) $2 b-a \in[n] \backslash A$. Thus $\gamma \leq 3\left|I_{1}\right|(n-|A|)$. The result follows.

Lemma 7.11. Let $r \geq 3, \delta=\frac{1}{34 \log _{2} n}, 0 \leq \xi \leq \frac{3}{5+8 \log _{2} r}$ and $A \subseteq[n]$ with $|A|=(1-\xi) n$. If there exists a good $r$-template $P$ of $A$ with $g(P, A)>2^{(1-\delta) n}$, then $\xi<\left(\log _{2} r-1\right) n^{-1 / 3} \log _{2} n+\delta$ and the number of integers $x \in A$ with $|P(x)| \geq 3$ is at most $n^{2 / 3} \log _{2} n$.

Proof. Let $X_{1}:=\{x \in A:|P(x)|=1\}, X_{2}:=\{x \in A:|P(x)|=2\}$ and $X_{3}:=$ $\{x \in A:|P(x)| \geq 3\}$. For $1 \leq i \leq 3$, let $x_{i}:=\left|X_{i}\right|$. Since $g(P, A)>2^{(1-\delta) n}$, we 
have $1^{x_{1}} \cdot 2^{x_{2}} \cdot r^{x_{3}}>2^{(1-\delta) n}$. Thus

$$
x_{3}>\frac{(\xi-\delta) n+x_{1}}{\log _{2} r-1} .
$$

We claim that $x_{2} \geq n / 4+\xi n+n / \log _{2} n+1$. Otherwise, we have $x_{1}+x_{3}>$ $3 n / 4-2 \xi n-n / \log _{2} n-1$, so $x_{3}>(9-13 \xi) n /\left(12 \log _{2} r\right)$ by inequality (7.2). Note that if $(a, b) \in X_{3}^{2}$ and $\{a, b, c\}$ forms a 3-AP for some $c \in A$, then $P$ contains a subtemplate $P^{\prime}$ such that $P^{\prime}$ is a rainbow 3-AP with $\left|P^{\prime}(a)\right|=$ $\left|P^{\prime}(b)\right|=\left|P^{\prime}(c)\right|=1$. By Proposition 7.9, we have

$$
\begin{aligned}
R(P) & \geq \frac{1}{3}\left(\left(\begin{array}{c}
x_{3} \\
2
\end{array}\right)-\frac{x_{3}^{2}}{4}-\frac{x_{3} \xi n}{2}\right)=\frac{1}{3}\left(\frac{x_{3}^{2}}{4}-\frac{x_{3} \xi n}{2}-\frac{x_{3}}{2}\right) \\
& >\frac{1}{3}\left(\frac{(9-13 \xi)^{2} n^{2}}{576 \log _{2}^{2} r}-\frac{(9-13 \xi) \xi n^{2}}{24 \log _{2} r}-\frac{(9-13 \xi) n}{24 \log _{2} r}\right) \\
& =\frac{1}{3}\left(\frac{(9-13 \xi) n^{2}}{576 \log _{2}^{2} r}\left(9-13 \xi-24 \xi \log _{2} r\right)-\frac{(9-13 \xi) n}{24 \log _{2} r}\right) \\
& \geq \frac{1}{3}\left(\frac{(9-13 \xi) n^{2}}{576 \log _{2}^{2} r}\left(9-\frac{3\left(13+24 \log _{2} r\right)}{5+8 \log _{2} r}\right)-\frac{(9-13 \xi) n}{24 \log _{2} r}\right) \\
& =\frac{1}{3}\left(\frac{6(9-13 \xi) n^{2}}{576\left(5+8 \log _{2} r\right) \log _{2}^{2} r}-\frac{(9-13 \xi) n}{24 \log _{2} r}\right)=\Theta\left(n^{2}\right),
\end{aligned}
$$

contradicting the assumption that $P$ is a good $r$-template.

For any $a \in X_{3}$, let $Y_{a}:=\left\{b \in X_{2}:\{a, b\}\right.$ is contained in some 3-AP of $\left.A\right\}$. For any $b \in X_{2} \backslash Y_{a}$, we have $2 a-b \notin A, 2 b-a \notin A$ and $(a+b) / 2 \notin A$. Then $\left|X_{2} \backslash Y_{a}\right| \leq\lceil n / 4\rceil+\xi n \leq n / 4+\xi n+1$. Thus $\left|Y_{a}\right| \geq x_{2}-n / 4-\xi n-1 \geq$ $n / \log _{2} n$. Note that if $a \in X_{3}, b \in X_{2}$ and $\{a, b, c\}$ forms a 3-AP for some $c \in A$, then $P$ contains a subtemplate $P^{\prime}$ such that $P^{\prime}$ is a rainbow 3-AP with $\left|P^{\prime}(a)\right|=\left|P^{\prime}(b)\right|=\left|P^{\prime}(c)\right|=1$. Thus $R(P) \geq x_{3} n /\left(2 \log _{2} n\right)$. Since $P$ is a good $r$-template, we have $n^{-1 / 3} f(n) \geq x_{3} n /\left(2 \log _{2} n\right)$. Thus $x_{3} \leq n^{2 / 3} \log _{2} n$. Moreover, by inequality (7.2), we have $\xi<\left(\log _{2} r-1\right) n^{-1 / 3} \log _{2} n+\delta$.

Lemma 7.12. Let $r \geq 3, \delta=\frac{1}{34 \log _{2} n}, 0 \leq \xi<\left(\log _{2} r-1\right) n^{-1 / 3} \log _{2} n+\delta$ and $A \subseteq[n]$ with $|A|=(1-\xi) n$. Suppose $P$ is a good $r$-template of $A$ with 
$g(P, A)>2^{(1-\delta) n}$. Then there exist two colors $i, j \in[r]$ such that the number of integers $x \in A$ with $P(x)=\{i, j\}$ is at least $(1-2 \delta) n$.

Proof. Let $X_{i}$ and $x_{i}(1 \leq i \leq 3)$ be the sets and values as given in the proof of Lemma 7.11. By Lemma 7.11 and inequality (7.2), we have $x_{3} \leq n^{2 / 3} \log _{2} n$ and $x_{1}+x_{3}<x_{3} \log _{2} r-(\xi-\delta) n$. Thus $x_{2}>(1-\delta) n-x_{3} \log _{2} r \geq(1-$ $\left.\delta-n^{-1 / 3} \log _{2} n \log _{2} r\right) n$. For $1 \leq i<j \leq r$, let $Y_{i, j}:=\left\{a \in X_{2}: P(a)=\{i, j\}\right\}$. Without loss of generality, let $\left|Y_{1,2}\right| \geq x_{2} /\left(\begin{array}{c}r \\ 2\end{array}\right)$. Let $Y^{\prime}=X_{2} \backslash Y_{1,2}$. Note that if $a \in Y_{1,2}, b \in Y^{\prime}$ and $\{a, b, c\}$ forms a 3-AP for some $c \in A$, then $P$ contains a subtemplate $P^{\prime}$ such that $P^{\prime}$ is a rainbow 3-AP with $\left|P^{\prime}(a)\right|=\left|P^{\prime}(b)\right|=$ $\left|P^{\prime}(c)\right|=1$. By Proposition 7.10 and since $P$ is a good $r$-template, we have $n^{-1 / 3} n^{2} / 4 \geq R(P) \geq\left(\left|Y_{1,2}\right|\left|Y^{\prime}\right| / 9-3 \xi n \cdot \min \left\{\left|Y_{1,2}\right|,\left|Y^{\prime}\right|\right\}\right) / 2$.

If $\left|Y_{1,2}\right| \leq\left|Y^{\prime}\right|$, then

$$
\begin{aligned}
\left|Y^{\prime}\right| & \leq \frac{9 n^{5 / 3}}{2\left|Y_{1,2}\right|}+27 \xi n \leq \frac{9 n^{5 / 3}\left(\begin{array}{l}
r \\
2
\end{array}\right)}{2 x_{2}}+27 \xi n \\
& <\frac{9 n^{2 / 3}\left(\begin{array}{l}
r \\
2
\end{array}\right)}{2\left(1-\delta-n^{-1 / 3} \log _{2} n \log _{2} r\right)}+27 \xi n=o(n) .
\end{aligned}
$$

On the other hand, $\left|Y^{\prime}\right| \geq x_{2} / 2>\left(1-\delta-n^{-1 / 3} \log _{2} n \log _{2} r\right) n / 2=\Theta(n)$, a contradiction. If $\left|Y_{1,2}\right| \geq\left|Y^{\prime}\right|$, then

$$
\begin{aligned}
\left|Y^{\prime}\right| & \leq \frac{n^{5 / 3}}{2\left(\left|Y_{1,2}\right| / 9-3 \xi n\right)} \leq \frac{n^{5 / 3}}{x_{2} / 9-6 \xi n} \\
& <\frac{n^{2 / 3}}{\left(1-\delta-n^{-1 / 3} \log _{2} n \log _{2} r\right) / 9-6 \xi} .
\end{aligned}
$$

Thus $\left|Y_{1,2}\right| \geq x_{2}-\left|Y^{\prime}\right| \geq(1-2 \delta) n$.

Lemma 7.13. Let $r \geq 3, \delta=\frac{1}{34 \log _{2} n}, 0 \leq \xi<\left(\log _{2} r-1\right) n^{-1 / 3} \log _{2} n+\delta$ and $A \subseteq[n]$ with $|A|=(1-\xi) n$. For any two colors $i, j \in[r]$, let $\mathscr{P}_{i, j}$ be the set of good $r$-templates of $A$, in which there are at least $(1-2 \delta) n$ integers with palette $\{i, j\}$. Then $g\left(\mathscr{P}_{i, j}, A\right) \leq 2^{|A|}\left(1+2^{-n / 240}\right)$. 
Proof. For any rainbow 3-AP-free $r$-coloring $\sigma \in G\left(\mathscr{P}_{i, j}, A\right)$, let $I_{\sigma}:=\{x \in$ $A: \sigma(x) \notin\{i, j\}\}$. Then $\left|I_{\sigma}\right| \leq 2 \delta n$. Let $\mathscr{G}_{0}:=\left\{\sigma \in G\left(\mathscr{P}_{i, j}, A\right):\left|I_{\sigma}\right|=0\right\}$ and $\mathscr{G}_{1}:=\left\{\sigma \in G\left(\mathscr{P}_{i, j}, A\right):\left|I_{\sigma}\right| \geq 1\right\}$. Then $g\left(\mathscr{P}_{i, j}, A\right)=\left|\mathscr{G}_{0}\right|+\left|\mathscr{G}_{1}\right| \leq 2^{|A|}+\left|\mathscr{G}_{1}\right|$.

Next, we consider the number of colorings in $\mathscr{G}_{1}$. We first choose a subset $I_{0} \subseteq A$ with $1 \leq\left|I_{0}\right| \leq 2 \delta n$. The number of options is at most $\sum_{1 \leq \ell \leq 2 \delta n}\left(\begin{array}{l}n \\ \ell\end{array}\right)$. We then use colors in $[r] \backslash\{i, j\}$ to color a fixed $I_{0}$. The number of colorings is at most $(r-2)^{2 \delta n}$. Now we consider the number of ways to color $A \backslash I_{0}$ using color $i$ or $j$. For any fixed $x \in I_{0}$, let $d(x)$ be the number of ordered pairs $(a, b) \in\left(A \backslash I_{0}\right)^{2}$ with $a<b$ such that $\{a, b, x\}$ forms a 3-AP. We claim that $d(x) \geq n / 5$. In fact, if we let $d_{1}:=\left|\left\{(a, b) \in A^{2}: x<a<b, x+b=2 a\right\}\right|$, $d_{2}:=\left|\left\{(a, b) \in A^{2}: a<x<b, a+b=2 x\right\}\right|$ and $d_{3}:=\mid\left\{(a, b) \in A^{2}: a<\right.$ $b<x, a+x=2 b\} \mid$, then $d(x) \geq d_{i}-2\left|I_{0}\right| \geq d_{i}-4 \delta$ n for $i \in\{1,3\}$, and $d(x) \geq d_{2}-\left|I_{0}\right| \geq d_{2}-2 \delta n$. Note that $d_{1} \geq\lfloor(n+x) / 2\rfloor-x-2 \xi n$, $d_{2} \geq \min \{x-1, n-x\}-\xi n$ and $d_{3} \geq x-\lceil(1+x) / 2\rceil-2 \xi n$. If $1 \leq x \leq 2 n / 5$, then $d(x) \geq d_{1}-4 \delta n \geq(n-x-1) / 2-2 \xi n-4 \delta n \geq n / 5$. If $2 n / 5<x \leq 3 n / 5$, then $d(x) \geq d_{2}-2 \delta n \geq\lfloor 2 n / 5\rfloor-\xi n-2 \delta n \geq n / 5$. If $3 n / 5<x \leq n$, then $d(x) \geq d_{3}-4 \delta n \geq(x-2) / 2-2 \xi n-4 \delta n \geq n / 5$. Hence, $d(x) \geq n / 5$. Note that if $(a, b) \in\left(A \backslash I_{0}\right)^{2}$ and $\{a, b, x\}$ forms a 3-AP, then $\sigma(a) \in\{i, j\}, \sigma(b) \in\{i, j\}$ and $\sigma(x) \in[r] \backslash\{i, j\}$, so $\sigma(a)=\sigma(b)$ in order to avoid a rainbow 3-AP. Thus the number of ways to color $A \backslash I_{0}$ is at most $2^{|A|-\left|I_{0}\right|-d(x) / 3} \leq 2^{|A|-n / 15}$.

Thus

$$
\begin{aligned}
g\left(\mathscr{P}_{i, j}, A\right) & \leq 2^{|A|}+\left|\mathscr{G}_{1}\right| \leq 2^{|A|}+\sum_{1 \leq \ell \leq 2 \delta n}\left(\begin{array}{l}
n \\
\ell
\end{array}\right)(r-2)^{2 \delta n} 2^{|A|-n / 15} \\
& \leq 2^{|A|}+2 \delta n \frac{n^{2 \delta n}}{(2 \delta n) !} r^{2 \delta n} 2^{|A|-n / 15} \\
& \leq 2^{|A|}+2^{2 \delta n \log _{2} n} 2^{2 \delta n \log _{2} r} 2^{|A|-n / 15} \\
& \leq 2^{|A|}+2^{n / 17+\left(n \log _{2} r\right) /\left(17 \log _{2} n\right)+|A|-n / 15} \leq 2^{|A|}+2^{|A|-n / 240} .
\end{aligned}
$$

The result follows.

Now we have all the ingredients to present our proof of Theorem 7.2.

Proof of Theorem 7.2. Let $\mathscr{C}$ be the collection of $r$-templates given by Theorem 7.8. Let $\delta=\left(34 \log _{2} n\right)^{-1}$, and let $\mathscr{C}_{1}:=\left\{P \in \mathscr{C}: g(P, A) \leq 2^{(1-\delta) n}\right\}$ and 
$\mathscr{C}_{2}:=\left\{P \in \mathscr{C}: g(P, A)>2^{(1-\delta) n}\right\}$. For each $P \in \mathscr{C}_{2}$, we have $|P(x)| \geq 1$ for every $x \in A$; otherwise $g(P, A)=0$. Moreover, we have $R(P) \leq n^{-1 / 3} f(n)$ by Theorem 7.8 (2). Thus every element $P \in \mathscr{C}_{2}$ is a good $r$-template of $A$. By Lemmas 7.11, 7.12 and 7.13, we have

$$
g\left(\mathscr{C}_{2}, A\right) \leq \sum_{1 \leq i<j \leq r} 2^{|A|}\left(1+2^{-n / 240}\right) \leq\left(\begin{array}{l}
r \\
2
\end{array}\right) 2^{|A|}\left(1+2^{-n / 240}\right) .
$$

By Theorem 7.8 (3), we have

$$
\begin{aligned}
g\left(\mathscr{C}_{1}, A\right) & \leq\left|\mathscr{C}_{1}\right| 2^{(1-\delta) n} \leq|\mathscr{C}| 2^{(1-\delta) n} \\
& \leq 2^{c n^{2 / 3} \log _{2}^{2} n+\left(1-1 /\left(34 \log _{2} n\right)\right) n}<2^{\left(1-1 /\left(35 \log _{2} n\right)\right) n} .
\end{aligned}
$$

Combining the above with Theorem 7.8 (1), the number of rainbow 3-AP-free $r$-colorings of $A$ is at most

$$
\begin{aligned}
g\left(\mathscr{C}_{1}, A\right)+g\left(\mathscr{C}_{2}, A\right) & \leq 2^{\left(1-1 /\left(35 \log _{2} n\right)\right) n}+\left(\begin{array}{l}
r \\
2
\end{array}\right) 2^{|A|}\left(1+2^{-n / 240}\right) \\
& \leq\left(\begin{array}{l}
r \\
2
\end{array}\right) 2^{|A|}+2^{-\frac{n}{36 \log _{2} n}} 2^{n} .
\end{aligned}
$$

This completes the proof of Theorem 7.2.

Next, we present our proof of Theorem 7.4.

Proof of Theorem 7.4. For any subset $A \subset[n]$ with $\left(2+8 \log _{2} r\right) n /\left(5+8 \log _{2} r\right)$ $\leq|A|<n$, by Theorem 7.2, we have $g_{r}(A) \leq\left(\begin{array}{l}r \\ 2\end{array}\right) 2^{n-1}+2^{-n /\left(36 \log _{2} n\right)} 2^{n} \leq$ $(r(r-1) / 4+o(1)) 2^{n}<\left(\begin{array}{c}r \\ 2\end{array}\right) 2^{n}-r^{2}+2 r \leq g_{r}([n])$. For any subset $A \subset[n]$ with $|A| \leq n / \log _{2} r$, we have $g_{r}(A) \leq r^{|A|} \leq 2^{|A| \log _{2} r} \leq 2^{n}<g_{r}([n])$. In the following, we may assume that $|A|=\alpha n$ with $1 / \log _{2} r<\alpha<\left(2+8 \log _{2} r\right) /(5+$ $\left.8 \log _{2} r\right)$.

Let $k:=n_{0}$, where $n_{0}$ is obtained by Theorem 7.2. Let $\delta=1 /\left(15 \log _{2}^{2} r\right)$ and $n \geq n_{1}=s z(k, \delta)$. By Szemerédi's Theorem, there are at least $(\alpha-\delta) n / k$ pairwise disjoint $k$-APs in $A$. For any $k$-AP $F=\{x, x+y, \ldots, x+(k-1) y\}$, let $\varphi$ be a mapping between $[k]$ and $F$ such that $\varphi(i)=x+(i-1) y$ for every $i \in[k]$. Then there is a bijection $\phi$ between all the 3-APs in $[k]$ and all 
the 3-APs in $F: \phi(\{a, b, c\})=\{\varphi(a), \varphi(b), \varphi(c)\}$ for any 3-AP $\{a, b, c\}$ in $[k]$. Therefore, there is a bijection between all the rainbow 3-AP-free $r$-colorings of $[k]$ and those of $F$, and thus $g_{r}(F)=g_{r}([k])$. By the choice of $k$, we have $g_{r}(F)=g_{r}([k]) \leq\left(\begin{array}{l}r \\ 2\end{array}\right) 2^{k}+2^{-k /\left(36 \log _{2} k\right)} 2^{k} \leq 2^{k+2 \log _{2} r}$. Then

$$
\begin{aligned}
g_{r}(A) & \leq r^{\delta n}\left(2^{k+2 \log _{2} r}\right)^{\frac{(\alpha-\delta) n}{k}}=2^{\delta n \log _{2} r} 2^{(\alpha-\delta) n+\frac{2(\alpha-\delta) n \log _{2} r}{k}} \\
& <2^{\alpha n+\delta n \log _{2} r+\frac{2 \alpha n \log _{2} r}{k}}<2^{\frac{\left(2+8 \log _{2} r\right) n}{5+8 \log _{2} r}+\frac{n}{15 \log _{2} r}+\frac{2 \alpha n \log _{2} r}{n_{0}}} \\
& <2^{\frac{\left(2+8 \log _{2} r\right) n}{5+8 \log _{2} r}+\frac{n}{14 \log _{2} r}}<2^{n}<g_{r}([n]) .
\end{aligned}
$$

This completes the proof of Theorem 7.4.

\subsection{Colorings of $\mathbb{Z}_{n}$ with no rainbow 3-AP}

We start by recalling the following known results.

Lemma 7.14 (Jungić et al. [103]). Let $n$ be a positive integer. Then aw $\left(\mathbb{Z}_{n}\right.$, $3)=3$ if and only if one of the following holds:

(1) $n$ is a power of 2 ;

(2) $n$ is prime and 2 is a generator of $\mathbb{Z}_{n}^{\times}$;

(3) $n$ is prime, $\operatorname{ord}_{n}(2)=\frac{n-1}{2}$, and $\frac{n-1}{2}$ is odd.

Lemma 7.15 (Butler et al. [29]). For any prime $p$, we have $3 \leq a w\left(\mathbb{Z}_{p}, 3\right) \leq 4$, and $a w\left(\mathbb{Z}_{p}, 3\right)=4$ implies that every rainbow 3-AP-free coloring of $\mathbb{Z}_{p}$ using exactly three colors contains a color which is used exactly once.

Now we have all the ingredients to present our proof of Theorem 7.6.

Proof of Theorem 7.6. If $a w\left(\mathbb{Z}_{p}, 3\right)=3$, then it suffices to show that $c \cdot \operatorname{ord}_{p}(2)$ $=p-1$. By Lemma 7.14, either $\operatorname{ord}_{p}(2)=p-1$, or $\operatorname{ord}_{p}(2)=(p-1) / 2$ and $(p-1) / 2$ is odd. In both cases, we have $c \cdot \operatorname{ord}_{p}(2)=p-1$.

If $a w\left(\mathbb{Z}_{p}, 3\right)=4$, then by Lemma 7.15, every rainbow 3-AP-free coloring of $\mathbb{Z}_{p}$ using exactly three colors contains a color which is used exactly once. Let $c$ 
be a rainbow 3-AP-free coloring of $\mathbb{Z}_{p}$ using exactly three colors. Without loss of generality, we may assume that $c(0)=1$ and $c(i) \in\{2,3\}$ for all $i \in \mathbb{Z}_{p} \backslash\{0\}$. In order to avoid a rainbow 3-AP, we have $c(i)=c(-i)$ and $c(i)=c(2 i)$ for each $i \in \mathbb{Z}_{p} \backslash\{0\}$.

For every integer $\ell$ with $1 \leq \ell \leq \frac{p-1}{2}$, let $Q_{\ell}:=\left\{\left(\ell \cdot 2^{i}\right) \bmod p: i \in\right.$ $\left.\mathbb{Z}^{+}\right\} \cup\left\{-\left(\ell \cdot 2^{i}\right) \bmod p: i \in \mathbb{Z}^{+}\right\}$. For every integer $j$ with $1 \leq j \leq \frac{p-1}{2}$, let $I_{1}:=\{1\}, I_{j}:=\{j\}$ if $j \geq 2$ and $j \notin \bigcup_{\ell \in \bigcup_{m=1}^{j-1} I_{m}} Q_{\ell}$, and $I_{j}:=\emptyset$ otherwise. Then for every $1 \leq \ell \leq \frac{p-1}{2}$, all the elements in $Q_{\ell}$ should be colored with the same color. For any $j$ with $2 \leq j \leq \frac{p-1}{2}$, if $I_{j}=\emptyset$, then $j \in Q_{\ell}$ for some $\ell$ with $\ell \leq j-1$ and $I_{\ell} \neq \emptyset$, which implies that $Q_{j} \subseteq Q_{\ell}$. Thus we only need to consider the sets $Q_{j}$ and $I_{j}$ with $I_{j} \neq \emptyset$. Moreover, for any $j_{1} \neq j_{2}$ with $I_{j_{1}} \neq \emptyset$ and $I_{j_{2}} \neq \emptyset$, we have $Q_{j_{1}} \cap Q_{j_{2}}=\emptyset$. Note that $\bigcup_{\ell \in \bigcup_{j=1}^{(p-1) / 2} I_{j}} Q_{\ell}=\mathbb{Z}_{p} \backslash\{0\}$. Furthermore, for any $\ell$ with $I_{\ell} \neq \emptyset$, we have $\left|Q_{\ell}\right|=\operatorname{ord}_{p}(2)$ if $\operatorname{ord}_{p}(2)$ is even, and $\left|Q_{\ell}\right|=2 \cdot \operatorname{ord}_{p}(2)$ if $\operatorname{ord}_{p}(2)$ is odd.

Next, we show that if $\{0, a, b\}$ forms a 3 -AP in $\mathbb{Z}_{p}$, then $a, b$ is contained in the same $Q_{j}$ for some $j$ with $1 \leq j \leq \frac{p-1}{2}$ and $I_{j} \neq \emptyset$. For a contradiction, suppose that $a \in Q_{j_{1}}$ and $b \in Q_{j_{2}}$ with $Q_{j_{1}} \cap Q_{j_{2}}=\emptyset$. Then $2 a(\bmod p) \in$ $Q_{j_{1}}, 2 b(\bmod p) \in Q_{j_{2}}$ and $-b(\bmod p) \in Q_{j_{2}}$. Since $\{0, a, b\}$ forms a 3-AP, we have $2 a \equiv b(\bmod p), a \equiv 2 b(\bmod p)$ or $a \equiv-b(\bmod p)$, which is impossible.

Therefore, we have

$$
\begin{aligned}
g_{r}\left(\mathbb{Z}_{p}\right) & =\left(\begin{array}{l}
r \\
2
\end{array}\right) 2^{p}-r^{2}+2 r+r\left(\begin{array}{c}
r-1 \\
2
\end{array}\right) p\left(2^{\sum_{j=1}^{(p-1) / 2}\left|I_{j}\right|}-2\right) \\
& =\left(\begin{array}{l}
r \\
2
\end{array}\right) 2^{p}-r^{2}+2 r+r\left(\begin{array}{c}
r-1 \\
2
\end{array}\right) p\left(2^{\frac{p-1}{c \cdot 0 d_{p}(2)}}-2\right) .
\end{aligned}
$$

This completes the proof of Theorem 7.6.

By Lemma $7.14(1)$, if $n$ is a power of 2 , then $g_{r}\left(\mathbb{Z}_{n}\right)=\left(\begin{array}{l}r \\ 2\end{array}\right) 2^{n}-r^{2}+2 r$. Note that every integer $n(\geq 3)$ can be decomposed into prime factors. Using Theorem 7.6, we can derive the following recurrence inequality. 
Corollary 7.16. For any positive integers $n \geq 1, r \geq 3$ and prime $p \geq 3$, we have

$$
\begin{aligned}
g_{r}\left(\mathbb{Z}_{n p}\right) \geq & \left(\begin{array}{l}
r \\
2
\end{array}\right) 2^{n p}-r^{2}+2 r+p r\left(g_{r}\left(\mathbb{Z}_{n}\right)-(r-1) 2^{n}+r-2\right) \\
& +\left(\begin{array}{l}
r \\
2
\end{array}\right) p\left(2^{\frac{p-1}{c \cdot 0 \text { ord } p(2)}}-2\right)\left(g_{r}\left(\mathbb{Z}_{n}\right)-2^{n}\right),
\end{aligned}
$$

where $c=1$ if $\operatorname{ord}_{p}(2)$ is even, and $c=2$ otherwise.

Proof. Note that there are exactly $\left(\begin{array}{l}r \\ 2\end{array}\right) 2^{n p}-r^{2}+2 r$ 2-colorings of $\mathbb{Z}_{n p}$. In the following, we will construct colorings using at least three colors. Let $R_{0}, R_{1}, \ldots, R_{p-1}$ be the residue classes modulo $p$ in $\mathbb{Z}_{n p}$.

We choose an arbitrary integer $q \in\{0,1, \ldots, p-1\}$ and an arbitrary color $\ell \in[r]$. Let $c_{n}$ be a rainbow 3-AP-free $r$-coloring of $\mathbb{Z}_{n}$ which contains at least two colors in $[r] \backslash\{\ell\}$. Let $c_{n p}$ be a coloring of $\mathbb{Z}_{n p}$ such that $c_{n p}(i p+q)=c_{n}(i)$ for $i \in\{0,1, \ldots, n-1\}$, and $c_{n p}(u)=\ell$ for any $u \in \mathbb{Z}_{n p} \backslash R_{q}$. Note that if $\{x, y, z\}$ forms a 3 -AP in $\mathbb{Z}_{n p}$ with $x, y \in R_{q}$, then $z \in R_{q}$ since $p$ is a prime. Thus $c_{n p}$ is a rainbow 3-AP-free $r$-coloring of $\mathbb{Z}_{n p}$. The number of such colorings $c_{n p}$ is $\operatorname{pr}\left(g_{r}\left(\mathbb{Z}_{n}\right)-r-(r-1)\left(2^{n}-2\right)\right)=\operatorname{pr}\left(g_{r}\left(\mathbb{Z}_{n}\right)-(r-1) 2^{n}+r-2\right)$.

If $a w\left(\mathbb{Z}_{p}, 3\right)=4$, then we can further construct another family of colorings. By Lemma 7.15, every rainbow 3-AP-free coloring of $\mathbb{Z}_{p}$ using exactly three colors contains a color which is used exactly once. We call the color used exactly once the special color, and the other two colors non-special. Let $c_{p}$ be a rainbow 3-AP-free coloring of $\mathbb{Z}_{p}$ using exactly three colors, and assume that the special color is used on $q$. Let $c_{n}$ be a rainbow 3-AP-free $r$-coloring of $\mathbb{Z}_{n}$ such that $c_{n}$ contains at least one color which is not one of the two non-special colors in $c_{p}$. Let $c_{n p}$ be a coloring of $\mathbb{Z}_{n p}$ such that $c_{n p}(i p+q)=c_{n}(i)$ for $i \in\{0,1, \ldots, n-1\}$, and $c_{n p}(u)=c_{p}(j)$ for $u \in R_{j}$ and $j \in\{0,1, \ldots, p-1\} \backslash\{q\}$. Note that if $\{x, y, z\}$ forms a 3 -AP in $\mathbb{Z}_{n p}$ with $x, y \in R_{i}$ for some $i \in\{0,1, \ldots, p-1\}$, then $z \in R_{i}$ since $p$ is a prime. Thus there is no 3 -AP in $\mathbb{Z}_{n p}$ such that the three elements are contained in exactly two different residue classes.

If $\{x, y, z\}$ forms a $3-\mathrm{AP}$ in $\mathbb{Z}_{n p}$ such that $x, y, z$ are contained in the same residue class, then this 3 -AP is not rainbow since $c_{n}$ is rainbow 3-AP-free. If 
$\{x, y, z\}$ forms a 3 -AP in $\mathbb{Z}_{n p}$ such that $x, y, z$ are contained in three different residue classes, then we may assume that $x=i_{1} p+j_{1}, y=i_{2} p+j_{2}, z=i_{3} p+j_{3}$ and $i_{1} p+j_{1}+i_{3} p+j_{3} \equiv 2\left(i_{2} p+j_{2}\right)(\bmod n p)$, where $0 \leq i_{1}, i_{2}, i_{3} \leq n-1$, $0 \leq j_{1}, j_{2}, j_{3} \leq p-1$, and $j_{1}, j_{2}, j_{3}$ are pairwise distinct. We may further assume that $i_{1} p+j_{1}+i_{3} p+j_{3}=2\left(i_{2} p+j_{2}\right)+k n p$ for some integer $k$. Thus $j_{1}+j_{3}-2 j_{2}=\left(2 i_{2}-i_{1}-i_{3}+k n\right) p$, which implies that $j_{1}+j_{3} \equiv 2 j_{2}(\bmod p)$ and thus $\left\{j_{1}, j_{2}, j_{3}\right\}$ forms 3 -AP in $\mathbb{Z}_{p}$. Then $\{x, y, z\}$ cannot form a rainbow 3-AP in $\mathbb{Z}_{n p}$ since $c_{p}$ is rainbow 3-AP-free with $c_{p}(q)$ being the special color. Thus $c_{n p}$ is a rainbow 3-AP-free $r$-coloring of $\mathbb{Z}_{n p}$. By Theorem 7.6, the number of such colorings $c_{n p}$ is $\frac{1}{r-2} r\left(\begin{array}{c}r-1 \\ 2\end{array}\right) p\left(2^{\frac{p-1}{c^{c \cdot o r d} p(2)}}-2\right)\left(g_{r}\left(\mathbb{Z}_{n}\right)-2^{n}\right)=$ $\left(\begin{array}{l}r \\ 2\end{array}\right) p\left(2^{\frac{p-1}{c \cdot \text { ord }_{p}(2)}}-2\right)\left(g_{r}\left(\mathbb{Z}_{n}\right)-2^{n}\right)$. The result follows.

Furthermore, we can prove the following result, using Corollary 7.16.

Corollary 7.17. For any integers $s \geq 0, m \geq 1, r \geq 3$ and primes $p_{1} \geq p_{2} \geq$ $\cdots \geq p_{m} \geq 3$, let $N=2^{s} \prod_{i=1}^{m} p_{i}$. Then $g_{r}\left(\mathbb{Z}_{N}\right)$ is at least

$$
\begin{aligned}
& \max _{\sigma}\left\{\left(\begin{array}{l}
r \\
2
\end{array}\right) 2^{2^{s} \prod_{i=1}^{m} q_{i}}-r^{2}+2 r\right. \\
& +\sum_{i=1}^{m}\left(\prod_{j=i}^{m}\left(q_{j} r+D_{j}\right)\right)\left(\left(\begin{array}{l}
r \\
2
\end{array}\right) 2^{\left.2^{s} \prod_{\ell=1}^{i-1} q_{\ell}-r^{2}+2 r\right)}\right. \\
& \left.+\sum_{i=1}^{m}\left(\prod_{j=i+1}^{m}\left(q_{j} r+D_{j}\right)\right)\left(-\left(q_{i} r(r-1)+D_{i}\right) 2^{2^{s}} \prod_{\ell=1}^{i-1} q_{\ell}+q_{i} r(r-2)\right)\right\},
\end{aligned}
$$

where the maximum is taken over all permutations $\sigma$ of $\left\{p_{1}, p_{2}, \ldots, p_{m}\right\}$, for every $j \in[m], q_{j}=\sigma\left(p_{j}\right), D_{j}=\left(\begin{array}{l}r \\ 2\end{array}\right) q_{j}\left(2^{\left(q_{j}-1\right) /\left(c_{j} \cdot \operatorname{ord}_{q_{j}}(2)\right)}-2\right), c_{j}=1$ if $\operatorname{ord}_{q_{j}}(2)$ is even, $c_{j}=2$ if $\operatorname{ord}_{q_{j}}(2)$ is odd, and define $\prod_{\ell=1}^{0} q_{\ell}=\prod_{j=m+1}^{m}\left(q_{j} r+D_{j}\right)=1$.

In particular, if aw $\left(\mathbb{Z}_{p_{i}}, 3\right)=3$ for all $i \in[m]$, then the maximum is attained when $\sigma$ is the identity permutation, and we have

$$
g_{r}\left(\mathbb{Z}_{N}\right) \geq\left(\begin{array}{l}
r \\
2
\end{array}\right) 2^{2^{s} \prod_{i=1}^{m} p_{i}}-r^{2}+2 r+\left(\begin{array}{c}
r-1 \\
2
\end{array}\right) \sum_{i=1}^{m}\left(\prod_{j=i}^{m} p_{j} r\right)\left(2^{2^{s} \prod_{\ell=1}^{i-1} p_{\ell}}-2\right) .
$$


Proof. The proof follows from Theorem 7.6, Lemma 7.14 (1), Corollary 7.16, and by induction on $m$. We omit the details. 


\section{Summary}

This thesis contains a number of new contributions to the area in discrete mathematics that is commonly known as Gallai-Ramsey theory, as well as some new results related to this research area, in particular dealing with the Erdős-Gyárfás function with respect to Gallai-colorings, and the rainbow Erdős-Rothschild problem with respect to 3-term arithmetic progressions.

One fundamental problem in the research of edge-colored graphs is to study the existence of nice substructures in an edge-colored host graph. In this thesis, the nice substructure we consider is either a rainbow subgraph or a monochromatic subgraph, and the host graph is a complete graph. The Gallai-Ramsey number $g r_{k}(G: H)$ is the minimum integer $n$ such that every $k$-edge-coloring of $K_{n}$ contains either a rainbow copy of $G$ or a monochromatic copy of $H$. This concept can be considered as a generalization of the classical Ramsey number.

In the study of Gallai-Ramsey numbers, most of the previous works focus on the case that $G$ is a triangle. An edge-coloring of a complete graph without rainbow triangles is called a Gallai-coloring, named after Tibor Gallai who proved his now well-known Gallai partition theorem for rainbow triangle-free colorings. In Chapters 2 and 3, we determine the exact values of the GallaiRamsey number $g r_{k}\left(K_{3}: H\right)$ for several choices of the graph $H$. These choices include the graph $K_{4}+e$ and all members of the class of connected graphs with five vertices and at most six edges. There are in total thirteen connected 5 -vertex graphs with at most six edges. The Gallai-Ramsey numbers for eight of these graphs have been determined in several papers by others, and our results cover all the remaining five graphs. We also obtain some exact values 
and bounds for the Ramsey numbers and Gallai-Ramsey numbers of a class of unicyclic graphs.

In Chapter 4, we consider two types of extremal problems related to Gallaicolorings. The first type of problem is to determine the maximum number of edges that are not contained in any rainbow triangle or monochromatic copy of $H$ in a $k$-edge-coloring of $K_{n}$. Using Szemerédi's Regularity Lemma and a variant of the Gallai-Ramsey number, we obtain upper and lower bounds for the maximum number of edges that are not contained in any rainbow triangle or monochromatic triangle in a $k$-edge-coloring of $K_{n}$. The second type of problem is the Gallai-Ramsey multiplicity problem. For $n \geq g r_{k}\left(K_{3}\right.$ : $K_{3}$ ), we determine upper and lower bounds for the minimum number of monochromatic triangles in a Gallai- $k$-coloring of $K_{n}$. We also obtain exact values of this number for some values of $k$ and $n$.

The Erdős-Gyárfás function $f(n, p, q)$ is defined as the minimum number of colors in an edge-coloring of $K_{n}$ such that every $K_{p}$ receives at least $q$ colors. This function was first introduced by Erdős and Shelah $[58,59]$ about 45 years ago, but Erdős and Gyárfás [62] were the first to study it in depth. In Chapter 5, combining the Erdős-Gyárfás function with Gallai-colorings, we consider the function $g(n, p, q)$, that is the minimum number of colors in a Gallai-coloring of $K_{n}$ such that every $K_{p}$ receives at least $q$ colors. Using the anti-Ramsey number for $K_{3}$, we show that $g(n, p, q)$ is nontrivial only for $2 \leq q \leq p-1$. We give a general lower bound for this function. We also study how this function falls off from being equal to $n-1$ when $q=p-1$ and $p \geq 4$ to being $\Theta(\log n)$ when $q=2$.

Let $n, k, m$ be positive integers with $n \gg m \gg k$, and let $\mathscr{G}$ be the set of connected graphs $G$ such that there is a $k$-connected monochromatic subgraph of order at least $n-f(G, k, m)$ in every rainbow $G$-free $m$-edge-coloring of $K_{n}$, where $f(G, k, m)$ does not depend on $n$. Fujita and Magnant [81] proved that $\mathscr{G}$ consists of precisely $K_{3}, P_{6}, P_{4}^{+}$and their connected subgraphs. Let $\mathscr{H}$ be the set of disconnected graphs satisfying the above property. In Chapter 6, we show that $\mathscr{G} \cup \mathscr{H}$ consists of precisely $P_{6}, P_{3} \cup P_{4}, K_{2} \cup P_{5}, K_{2} \cup 2 P_{3}, 2 K_{2} \cup K_{3}$, $2 K_{2} \cup P_{4}^{+}, 3 K_{2} \cup K_{1,3}$ and their subgraphs. We also present a forbidden rainbow subgraph condition for an edge-colored complete bipartite graph to have a highly-connected monochromatic subgraph. 
The rainbow Erdős-Rothschild problem asks which graph on $n$ vertices admits the maximum number of $r$-edge-colorings without rainbow copies of $H$. In Chapter 7, we deal with the rainbow Erdős-Rothschild problem with respect to 3-term arithmetic progressions. We prove that $[n]$ attains the maximum number of rainbow 3-term arithmetic progression-free $r$-colorings among all subsets of $[n]$. Moreover, the asymptotic number of $r$-colorings of $[n]$ without rainbow 3-term arithmetic progressions is obtained, and we show that the typical colorings with this property are 2-colorings. We also obtain the exact number of rainbow 3-term arithmetic progression-free $r$ colorings of $\mathbb{Z}_{p}$, where $p$ is any prime and $\mathbb{Z}_{p}$ is the cyclic group of order $p$. The proof technique we used in this final chapter is based on the Hypergraph Container Method which was developed by Balogh-Morris-Samotij [15] and Saxton-Thomason [160], independently.

Throughout this thesis, we present several open problems and conjectures that remain unsolved. In particular, a driving problem is to determine the Gallai-Ramsey numbers for complete graphs (see Conjecture 1.1). This problem is related to the classical 2-colored Ramsey numbers for complete graphs, and also has a close relationship with the multicolor Erdős-Hajnal conjecture. Another important problem is to study how does the additional constraint on rainbow triangles influence the classical extremal problems, such as the multicolor Ramsey number and the Erdős-Gyárfás function. Although we have provided some comparisons and analyses in Sections 3.4 and 5.9, further explorations in this direction could be fruitful. 



\section{Samenvatting}

Dit proefschrift bevat een aantal nieuwe bijdragen op het deelgebied van de discrete wiskunde dat bekend staat als Gallai-Ramsey theorie, alsmede een aantal nieuwe resultaten op gerelateerde deelgebieden, bijvoorbeeld met betrekking tot de Erdős-Gyárfás functie toegepast op Gallai-kleuringen, en het regenboog Erdős-Rothschild probleem toegepast op arithmetische progressies met drie termen.

Een van de fundamentele problemen in het onderzoek aan grafen met een lijnkleuring richt zich op de existentie van mooie deelstructuren in een lijngekleurde waardgraaf. De mooie deelstructuren waar we ons in dit proefschrift op richten zijn ofwel regenboog deelgrafen, ofwel monochromatische deelgrafen, terwijl de waardgraaf in dit geval een volledige graaf is. Het Gallai-Ramsey getal $g r_{k}(G: H)$ is het kleinste gehele getal $n$ waarvoor geldt dat elke $k$-lijnkleuring van $K_{n}$ ofwel een regenboog kopie van $G$ bevat, ofwel een monochromatische kopie van $H$. Dit concept kan opgevat worden als een generalisatie van het klassieke Ramsey getal.

Eerder onderzoek aan Gallai-Ramsey getallen heeft zich hoofdzakelijk gericht op het geval dat $G$ een driehoek is. Een lijnkleuring van een volledige graaf zonder regenboog driehoeken wordt wel een Gallai-kleuring genoemd, als hommage aan Tibor Gallai voor zijn bewijs van een nu welbekend resultaat over Gallai-partities van regenboog driehoekvrije kleuringen. In Hoofdstuk 2 en Hoofdstuk 3 bepalen we de exacte waarde van het Gallai-Ramsey getal $g r_{k}\left(K_{3}: H\right)$ voor verschillende keuzes van $H$, met name voor de graaf $K_{4}+e$ en alle samenhangende grafen op 5 punten en hooguit 6 lijnen. Er bestaan in totaal 13 van die grafen. Voor 8 van die grafen zijn de Gallai-Ramsey getallen 
in eerder werk van anderen bepaald. In dit proefschrift worden de resterende 5 gevallen behandeld. Tevens leiden we exacte waarden en grenzen af voor de Ramsey getallen en Gallai-Ramsey getallen voor een klasse van grafen met een unieke cykel.

In Hoofdstuk 4 richten we ons op twee typen van extremaalproblemen met betrekking tot Gallai-kleuringen. Het eerste type probleem draait om het bepalen van het maximale aantal lijnen dat niet in een regenboog driehoek of monochromatische kopie van $H$ bevat is in een $k$-lijnkleuring van $K_{n}$. We bepalen onder - en bovengrenzen voor dit aantal, met gebruikmaking van het Regulariteitslemma van Szemerédi en een variant van het Gallai-Ramsey getal. Het tweede type probleem staat bekend als het Gallai-Ramsey multipliciteitsprobleem. Voor $n \geq g r_{k}\left(K_{3}: K_{3}\right)$ bepalen we onder - en bovengrenzen voor het minimale aantal monochromatische driehoeken in een Gallai- $k$-kleuring van $K_{n}$. Tevens leiden we een aantal exacte waarden voor dit aantal af voor specifieke waarden van $k$ en $n$.

De Erdős-Gyárfás functie $f(n, p, q)$ is gedefinieerd als het minimale aantal kleuren in een lijnkleuring van $K_{n}$ waarin elke $K_{p}$ minstens $q$ kleuren heeft. Deze functie is ongeveer 45 jaar geleden ingevoerd door Erdős en Shelah $[58,59]$, maar pas veel later echt tot zekere diepte bestudeerd door Erdős and Gyárfás [62]. In Hoofdstuk 5 koppelen we de Erdős-Gyárfás functie aan Gallai-kleuringen, en richten we ons op de functie $g(n, p, q)$, te weten het minimale aantal kleuren in een Gallai-kleuring van $K_{n}$ waarin elke $K_{p}$ minstens $q$ kleuren heeft. Met gebruikmaking van het anti-Ramsey getal van $K_{3}$ laten we zien dat $g(n, p, q)$ alleen zinvol is voor $2 \leq q \leq p-1$. We leiden een ondergrens af voor deze functie, en we bestuderen het verloop van deze functie die gelijk is aan $n-1$ als $q=p-1$ en $p \geq 4$, maar slechts $\Theta(\log n)$ als $q=2$.

Laat $n, k, m$ positieve getallen zijn met $n \gg m \gg k$, en laat $\mathscr{G}$ bestaan uit de samenhangende grafen $G$ waarvoor geldt dat er een $k$-samenhangende monochromatische deelgraaf met tenminste $n-f(G, k, m)$ punten bestaat in elke regenboog $G$-vrije $m$-lijnkleuring van $K_{n}$, waarbij $f(G, k, m)$ niet afhangt van $n$. Fujita and Magnant [81] hebben bewezen dat $\mathscr{G}$ precies bestaat uit $K_{3}$, $P_{6}, P_{4}^{+}$en hun samenhangende deelgrafen. Laat $\mathscr{H}$ bestaan uit alle onsamenhangende grafen met de bovengenoemde eigenschap. In Hoofdstuk 6 laten 
we zien dat $\mathscr{G} \cup \mathscr{H}$ precies bestaat uit $P_{6}, P_{3} \cup P_{4}, K_{2} \cup P_{5}, K_{2} \cup 2 P_{3}, 2 K_{2} \cup K_{3}$, $2 K_{2} \cup P_{4}^{+}, 3 K_{2} \cup K_{1,3}$ en al hun deelgrafen. We presenteren ook een voorwaarde in termen van een verboden regenboog deelgraaf die impliceert dat een lijngekleurde volledige bipartiete graaf een monochromatische deelgraaf met een hoge samenhang heeft.

De regenboogvariant van het Erdős-Rothschild probleem houdt in welke graaf op $n$ punten het maximale aantal $r$-lijnkleuringen zonder een regenboog kopie van $H$ heeft. In Hoofdstuk 7 richten we ons op dit regenboog ErdősRothschild probleem met betrekking tot arithmetische progressies met 3 termen. We bewijzen dat van alle deelverzamelingen van $[n]$, $[n]$ zelf het maximale aantal $r$-kleuringen bevat die vrij zijn van regenboog arithmetische progressies met 3 termen. Tevens bepalen we het asymptotische aantal van dergelijke $r$-kleuringen, en we laten zien dat de typische kleuringen 2-kleuringen zijn. Tot slot bepalen we het exacte aantal van dergelijke $r$ kleuringen voor $\mathbb{Z}_{p}$, de cyclische groep van orde $p$ waarbij $p$ een priemgetal is. We gebruiken in dit laatste hoofdstuk een bewijstechniek die gebaseerd is op de Hypergraaf Containermethode die onafhankelijk door Balogh-MorrisSamotij [15] en Saxton-Thomason [160] ontwikkeld is.

Naast de bovengenoemde resultaten bevat dit proefschrift verscheidene problemen en vermoedens die tot op heden onopgelost zijn gebleven. Eén van de drijvende problemen in dit gebied is het bepalen van de Gallai-Ramsey getallen van volledige grafen (zie Conjecture 1.1). Dit probleem is verwant aan de klassieke Ramsey getallen voor 2-kleuringen van volledige grafen, en tevens aan het vermoeden van Erdős-Hajnal voor meerdere kleuren. Een ander belangrijk probleem betreft het bepalen in welke mate klassieke extremaalproblemen worden beïnvloed door de bijkomende voorwaarde op regenboog driehoeken, bijvoorbeeld in het geval van het veelkleurige Ramsey getal en de Erdős-Gyárfás functie. Hoewel we in Sectie 3.4 en Sectie 5.9 van het proefschrift enkele vergelijkingen en analyses op dit gebied verschaffen, zou een dieper onderzoek wellicht zijn vruchten kunnen afwerpen. 



\section{Bibliography}

[1] N. Alon, P. Erdős, D.S. Gunderson and M. Molloy, A Ramsey-type problem and the Turán numbers, J. Graph Theory 40 (2002), 120-129.

[2] N. Alon and W. Maass, Meanders, Ramsey theory and lower bounds for branching programs, Proc. 27th IEEE FOCS (1986), pp. 410-417.

[3] M. Axenovich, A generalized Ramsey problem, Discrete Math. 222 (2000), 247-249.

[4] M. Axenovich and D. Fon-Der-Flaass, On rainbow arithmetic progressions, Electron. J. Combin. 11 (2004), Research Paper 1, 7pp.

[5] M. Axenovich, Z. Füredi and D. Mubayi, On generalized Ramsey theory: the bipartite case, J. Combin. Theory Ser. B 79 (2000), 66-86.

[6] M. Axenovich and P. Iverson, Edge-colorings avoiding rainbow and monochromatic subgraphs, Discrete Math., 308 (2008), 4710-4723.

[7] M. Axenovich and A. Kündgen, On a generalized anti-Ramsey problem, Combinatorica 21 (2001), 335-349.

[8] M. Balko, V. Jelínek and P. Valtr, On ordered Ramsey numbers of boundeddegree graphs, J. Combin. Theory Ser. B 134 (2019), 179-202.

[9] J. Balogh, S. English, E. Heath and R.A. Krueger, Lower bounds on the Erdős-Gyárfás problem via color energy graphs, arXiv:2102.11466v1, (2021). 
[10] J. Balogh and L.N. Li, The typical structure of Gallai colorings and their extremal graphs, SIAM J. Discrete Math. 33 (2019), 2416-2443.

[11] J. Balogh, H. Liu, Š. Petříčková and M. Sharifzadeh, The typical structure of maximal triangle-free graphs, Forum Math. Sigma 3 (2015), Paper No. e20, 19 pp.

[12] J. Balogh, H. Liu and M. Sharifzadeh, The number of subsets of integers with no k-term arithmetic progression, Int. Math. Res. Not. 2017 (2017), 6168-6186.

[13] J. Balogh, H. Liu, M. Sharifzadeh and A. Treglown, The number of maximal sum-free subsets of integers, Proc. Amer. Math. Soc. 143 (2015), 4713-4721.

[14] J. Balogh, H. Liu, M. Sharifzadeh and A. Treglown, Sharp bound on the number of maximal sum-free subsets of integers, J. Eur. Math. Soc. 20 (2018), 1885-1911.

[15] J. Balogh, R. Morris and W. Samotij, Independent sets in hypergraphs, $J$. Amer. Math. Soc. 28 (2015), 669-709.

[16] J. Balogh, R. Morris, W. Samotij and L. Warnke, The typical structure of sparse $K_{r+1}$-free graphs, Trans. Amer. Math. Soc. 368 (2016), 64396485.

[17] J.O. Bastos, F.S. Benevides and J. Han, The number of Gallai $k$-colorings of complete graphs, J. Combin. Theory Ser. B 144 (2020), 1-13.

[18] G. Beam and M. Budden, Weakened Gallai-Ramsey numbers, Surv. Math. Appl. 13 (2018), 131-145.

[19] Z. Berikkyzy, A. Schulte and M. Young, Anti-van der Waerden numbers of 3-term arithmetic progressions, Electron. J. Combin. 24 (2017), Paper No. 2.39, 9pp.

[20] T. Bohman and P. Keevash, The early evolution of the $H$-free process, Invent. Math. 181 (2010), 291-336. 
[21] B. Bollobás and A. Gyárfás, Highly connected monochromatic subgraphs, Discrete Math. 308 (2008), 1722-1725.

[22] J.A. Bondy and U.S.R. Murty, Graph Theory, Graduate Texts in Mathematics, Volume 244, Springer, New York (2008).

[23] C. Bosse, Hadwiger numbers and Gallai-Ramsey numbers of special graphs, PhD thesis, University of Central Florida (2019).

[24] C.W. Bostwick, J.W. Andrushkiw, H. Goheen, F.E. Clark and P. Treuenfels, Problems for solution: E1321-E1325, Amer. Math. Monthly 65 (1958), 446-447.

[25] W.G. Brown, P. Erdős and V.T. Sós, Some extremal problems on $r$-graphs, in: New directions in the theory of graphs (Proc. Third Ann Arbor Conf., Univ. Michigan, Ann Arbor, Mich., 1971), Academic Press, New York, (1973), 53-63.

[26] D. Bruce and Z.-X. Song, Gallai-Ramsey numbers of $C_{7}$ with multiple colors, Discrete Math. 342 (2019), 1191-1194.

[27] M. Budden, J. Hiller and A. Penland, Constructive method in GallaiRamsey theory for hypergraphs, Integers 20A (2020), \#A4.

[28] S.A. Burr and J.A. Roberts, On Ramsey numbers for stars, Utilitas Math. 4 (1973), 217-220.

[29] S. Butler, C. Erickson, L. Hogben, K. Hogenson, L. Kramer, R. Kramer, J. Lin, R. Martin, D. Stolee, N. Warnberg and Michael Young, Rainbow arithmetic progressions, J. Comb. 7 (2016), 595-626.

[30] A. Cameron, An explicit edge-coloring of $K_{n}$ with six colors on every $K_{5}$, Electron. J. Combin. 26 (2019), Paper No. 4.13, 20 pp.

[31] K. Cameron, J. Edmonds and L. Lovász, A note on perfect graphs, Period. Math. Hungar. 17 (1986), 173-175.

[32] P. Cameron and P. Erdős, On the number of sets of integers with various properties, In Number Theory, edited by R.A. Mollin, 61-79. Walter de Gruyter, Berlin, 1990. 
[33] A. Cameron and E. Heath, A $(5,5)$-colouring of $K_{n}$ with few colours, Combin. Probab. Comput. 27 (2018), 892-912.

[34] M. Chen, Y.S. Li and C.P. Pei, Gallai-Ramsey numbers of odd cycles and complete bipartite graphs, Graphs Combin. 34 (2018) 1185-1196.

[35] Y.Y. Cheng, Y.F. Jing, L.N. Li, G.H. Wang and W.L Zhou, Integer colorings with forbidden rainbow sums, arXiv:2005.14384v1, (2020).

[36] F.R.K. Chung and R.L. Graham, Edge-colored complete graphs with precisely colored subgraphs, Combinatorica 3 (1983), 315-324.

[37] V. Chvátal and F. Harary, Generalized Ramsey theory for graphs, Bull. Amer. Math. Soc. 78 (1972), 423-426.

[38] V. Chvátal and F. Harary, Generalized Ramsey theory for graphs II, small diagonal numbers, Proc. Amer. Math. Soc. 32 (1972), 389-394.

[39] V. Chvátal and F. Harary, Generalized Ramsey theory for graphs III, small off-diagonal numbers, Pacific J. Math. 41 (1972), 335-345.

[40] V. Chvátal and F. Harary, Generalized Ramsey theory for graphs I, diagonal numbers, Period. Math. Hungar. 3 (1973), 115-124.

[41] M. Clancy, Some small Ramsey numbers, J. Graph Theory 1 (1977), 89-91.

[42] E.J. Cockayne and P.J. Lorimer, The Ramsey number for stripes, J. Austral. Math. Soc. 19 (1975), 252-256.

[43] M. Codish, M. Frank, A. Itzhakov and A. Miller, Computing the Ramsey number $R(4,3,3)$ using abstraction and symmetry breaking, Constraints 21 (2016), 375-393.

[44] D. Conlon, A new upper bound for diagonal Ramsey numbers, Ann. of Math. 170 (2009), 941-960.

[45] D. Conlon, On the Ramsey multiplicity of complete graphs, Combinatorica 32 (2012), 171-186. 
[46] D. Conlon and A. Ferber, Lower bounds for multicolor Ramsey numbers, Adv. Math. 378 (2021), 107528.

[47] D. Conlon, J. Fox, C. Lee and B. Sudakov, On the grid Ramsey problem and related questions, Int. Math. Res. Not. 2015 (2015), 8052-8084.

[48] D. Conlon, J. Fox, C. Lee and B. Sudakov, The Erdős-Gyárfás problem on generalized Ramsey numbers, Proc. London Math. Soc. 110 (2015), $1-18$.

[49] D. Conlon, J. Fox, C. Lee and B. Sudakov, Ordered Ramsey numbers, J. Combin. Theory Ser. B 122 (2017), 353-383.

[50] D. Conlon, J. Fox and B. Sudakov, Hypergraph Ramsey numbers, J. Amer. Math. Soc. 23 (2010), 247-266.

[51] D. Conlon, J. Fox and B. Sudakov, Recent developments in graph Ramsey theory, in: Surveys in Combinatorics 2015, Cambridge Univ. Press, (2015), 49-118.

[52] J. Cummings, D. Král', F. Pfender, K. Sperfeld, A. Treglown and M. Young, Monochromatic triangles in three-coloured graphs, J. Combin. Theory Ser. B 103 (2013), 489-503.

[53] E. Egerváry, On combinatorial properties of matrices, Mat. Lapok. 38 (1931), 16-28.

[54] D. Eichhorn and D. Mubayi, Edge-coloring cliques with many colors on subcliques, Combinatorica 20 (2000), 441-444.

[55] P. Erdős, On sets of distances of $n$ points, Amer. Math. Monthly 53 (1946), 248-250.

[56] P. Erdős, Some remarks on the theory of graphs, Bull. Amer. Math. Soc. 53 (1947), 292-294.

[57] P. Erdős, Some new applications of probability methods to combinatorial analysis and graph theory, In Proceedings of the 5th Southeastern Conference on Combinatorics, Graph Theory and Computing, Boca Raton, FL, (1974), 39-51. 
[58] P. Erdős, Problems and results on finite and infinite graphs, in: Recent advances in graph theory (Proc. Second Czechoslovak Sympos., Prague, 1974), Academia, Prague, (1975), 183-192.

[59] P. Erdős, Solved and unsolved problems in combinatorics and combinatorial number theory, Congr. Numer. 32 (1981), 49-62.

[60] P. Erdős and T. Gallai, On maximal paths and circuits of graphs, Acta Math. Acad. Sci. Hungar. 10 (1959), 337-356.

[61] P. Erdős and R.L. Graham, Old and new problems and results in combinatorial number theory, Monographies de L'Enseignement Mathématique, 28. Université de Genève, L’Enseignement Mathématique, Geneva, 1980, $128 \mathrm{pp}$.

[62] P. Erdős and A. Gyárfás, A variant of the classical Ramsey problem, Combinatorica 17 (1997), 459-467.

[63] P. Erdős and A. Hajnal, Ramsey-type theorems, Discrete Appl. Math. 25 (1989), 37-52.

[64] P. Erdős, A. Hajnal and R. Rado, Partition relations for cardinal numbers, Acta Math. Acad. Sci. Hungar. 16 (1965), 93-196.

[65] P. Erdős, D.J. Kleitman and B.L. Rothschild, Asymptotic enumeration of $K_{n}$-free graphs, Colloquio Internazionale sulle Teorie Combinatorie (Rome, 1973), Tomo II, pp. 19-27. Atti dei Convegni Lincei, No. 17, Accad. Naz. Lincei, Rome, 1976.

[66] P. Erdős and L. Lovász, Problems and results on 3-chromatic hypergraphs and some related questions, in: Infinite and Finite Sets (A. Hajnal, R. Rado, V.T. Sós eds.), Colloq. Math. Soc. J. Bolyai, North-Holland, (1975), 609-627.

[67] P. Erdős, A. Simonovits and V.T. Sós, Anti-Ramsey theorems, in: Infnite and Finite Sets (A. Hajnal, R. Rado, V.T. Sós eds.), Colloq. Math. Soc. J. Bolyai, North-Holland, (1975), 633-643. 
[68] P. Erdős and G. Szekeres, A combinatorial problem in geometry, Compositio Math. 2 (1935), 463-470.

[69] P. Erdős and P. Turán, On some sequences of integers, J. London Math. Soc. 11 (1936), 261-264.

[70] L. Eroh, Rainbow Ramsey numbers, PhD thesis, Western Michigan University (2000).

[71] V. Falgas-Ravry, K. O'Connell and A. Uzzell, Multicolour containers, extremal entropy, and counting, Random Struct. Alg. 54 (2019), 676720 .

[72] R.J. Faudree, R. Gould, M. Jacobson and C. Magnant, Ramsey numbers in rainbow triangle free colorings, Australas. J. Combin. 46 (2010), 269-284.

[73] S. Fish, C. Pohoata and A. Sheffer, Local properties via color energy graphs and forbidden configurations, SIAM J. Discrete Math. 34 (2020), 177-187.

[74] J. Fox, There exist graphs with super-exponential Ramsey multiplicity constant, J. Graph Theory 57 (2008), 89-98.

[75] J. Fox, A. Grinshpun and J. Pach, The Erdős-Hajnal conjecture for rainbow triangles, J. Combin. Theory Ser. B 111 (2015), 75-125.

[76] J. Fox, J. Pach and A. Suk, The Schur-Erdős problem for semi-algebraic colorings, Israel J. Math. 239 (2020), 39-57.

[77] J. Fox and B. Sudakov, Ramsey-type problem for an almost monochromatic $K_{4}$, SIAM J. Discrete Math. 23 (2009), 155-162.

[78] S. Fujita and C. Magnant, Gallai-Ramsey numbers for cycles, Discrete Math. 311 (2011), 1247-1254.

[79] S. Fujita and C. Magnant, Note on highly connected monochromatic subgraphs in 2-colored complete graphs, Electron. J. Combin. 18 (2011), Paper 15, 5 pp. 
[80] S. Fujita and C. Magnant, Extensions of Gallai-Ramsey results, J. Graph Theory 70 (2012), 404-426.

[81] S. Fujita and C. Magnant, Forbidden rainbow subgraphs that force large highly connected monochromatic subgraphs, SIAM J. Discrete Math. 27 (2013), 1625-1637.

[82] S. Fujita, C. Magnant and K. Ozeki, Rainbow generalizations of Ramsey theory: a survey, Graphs Combin. 26 (2010), 1-30.

[83] S. Fujita, C. Magnant and K. Ozeki, Rainbow generalizations of Ramsey theory: a dynamic survey, Theory Appl. Graphs 0 (2014), Article 1.

[84] H. Furstenberg, Recurrence in ergodic theory and combinatorial number theory, Princeton University Press, Princeton, N.J. (1981).

[85] T. Gallai, Transitiv orientierbare Graphen, Acta Math. Acad. Sci. Hungar. 18 (1967), 25-66.

[86] A.W. Goodman, On sets of acquaintances and strangers at any party, Amer. Math. Monthly 66 (1959), 778-783.

[87] R.L. Graham, B.L. Rothschild and J.H. Spencer, Ramsey Theory, 2nd Edition, John Wiley \& Sons, New York (1990).

[88] R.E. Greenwood and A.M. Gleason, Combinatorial relations and chromatic graphs, Canadian J. Math. 7 (1955), 1-7.

[89] L. Guth and N.H. Katz, On the Erdős distinct distances problem in the plane, Ann. of Math. 181 (2015), 155-190.

[90] A. Gyárfás, Partition coverings and blocking sets in hypergraphs (in Hungarian), Comm. Comp. Automat. Inst. Hung. Acad. Sci. 71 (1977), 62pp.

[91] A. Gyárfás, G.N. Sárközy, A. Sebő and S. Selkow, Ramsey-type results for Gallai colorings, J. Graph Theory 64 (2010), 233-243.

[92] A. Gyárfás and G. Simonyi, Edge colorings of complete graphs without tricolored triangles, J. Graph Theory 46 (2004), 211-216. 
[93] M. Hall, C. Magnant, K. Ozeki and M. Tsugaki, Improved upper bounds for Gallai-Ramsey numbers of paths and cycles, J. Graph Theory 75 (2014), 59-74.

[94] H. Hàn and A. Jiménez, Maximum number of sum-free colorings in finite abelian groups, Israel J. Math. 226 (2018), 505-534.

[95] H. Harborth and I. Mengersen, All Ramsey numbers for five vertices and seven or eight edges, Discrete Math. 73 (1988/89), 91-98.

[96] H. Hatami, J. Hladký, D. Král', S. Norine and A. Razborov, Non-threecolourable common graphs exist, Combin. Probab. Comput. 21 (2012), 734-742.

[97] G.R.T. Hendry, Ramsey numbers for graphs with five vertices, J. Graph Theory 13 (1989), 245-248.

[98] D. Hilbert, Ueber die Irreducibilität ganzer rationaler Functionen mit ganzzahligen Coefficienten, J. Reine Angew. Math. 110 (1892), 104-129.

[99] C. Hoppen, Y. Kohayakawa and H. Lefmann, Hypergraphs with many Kneser colorings, European J. Combin. 33 (2012), 816-843.

[100] C. Hoppen, H. Lefmann and K. Odermann, A rainbow Erdős-Rothschild problem, SIAM J. Discrete Math. 31 (2017), 2647-2674.

[101] C. Hoppen, H. Lefmann and K. Odermann, On graphs with a large number of edge-colorings avoiding a rainbow triangle, European $J$. Combin. 66 (2017), 168-190.

[102] M. Jenssen and J. Skokan, Exact Ramsey numbers of odd cycles via nonlinear optimisation, Adv. Math. 376 (2021), Paper No. 107444, 46 pp.

[103] V. Jungić, J. Licht, M. Mahdian, J. Nešetřil and R. Radoičić, Rainbow arithmetic progressions and anti-Ramsey results, Combin. Probab. Comput. 12 (2003), 599-620. 
[104] M. Kano and X.L. Li, Monochromatic and heterochromatic subgraphs in edge-colored graphs-a survey, Graphs Combin. 24 (2008), 237-263.

[105] R. Katić, C. Magnant and P.S. Nowbandegani, Forbidden properly edgecolored subgraphs that force large highly connected monochromatic subgraphs, Graphs Combin. 33 (2017), 969-979.

[106] G.O.H. Katona, C. Magnant, Y.P. Mao and Z. Wang, Gallai Ramsey number for double stars, arXiv:2001.02789v1, (2020).

[107] P. Keevash and B. Sudakov, On the number of edges not covered by monochromatic copies of a fixed graph, J. Combin. Theory Ser. B 90 (2004), 41-53.

[108] J. Komlós and M. Simonovits, Szemerédi’s Regularity Lemma and its applications in graph theory, in: Combinatorics, Paul Erdös is Eighty, Vol. 2, Keszthely, 1993, in: Bolyai Soc. Math. Stud., Vol. 2, János Bolyai Math. Soc, Budapest, 1996, pp. 295-352.

[109] D. König, Gráfok és Mátrixok, Matematikai és Fizikai Lapok 38 (1931), 116-119.

[110] D. König, Theorie der endlichen und unendlichen Graphen, Akademische Verlagsgesellschaft (1936).

[111] J. Körner and G. Simonyi, Graph pairs and their entropies: modularity problems, Combinatorica 20 (2000), 227-240.

[112] J. Körner, G. Simonyi and Z. Tuza, Perfect couples of graphs, Combinatorica 12 (1992), 179-192.

[113] A. Kostochka and D. Mubayi, When is an almost monochromatic $K_{4}$ guaranteed? Combin. Probab. Comput. 17 (2008), 823-830.

[114] R.A. Krueger, Generalized Ramsey numbers: forbidding paths with few colors, Electron. J. Combin. 27 (2020), Paper No. 1.44, 10 pp.

[115] D. Kühn, D. Osthus, T. Townsend and Y. Zhao, On the structure of oriented graphs and digraphs with forbidden tournaments or cycles, $J$. Combin. Theory Ser. B 124 (2017), 88-127. 
[116] H. Lei, Y.T. Shi, Z.-X. Song and J.M. Zhang, Gallai-Ramsey numbers of $C_{10}$ and $C_{12}$, Australas. J. Combin. 79 (2021), 380-400.

[117] X.H. Li, P. Besse, C. Magnant, L.G. Wang and N. Watts, Gallai-Ramsey numbers for rainbow paths, Graphs Combin. 36 (2020), 1163-1175.

[118] Y.S. Li, C.C. Rousseau and W.A. Zang, Asymptotic upper bounds for Ramsey functions, Graphs Combin. 17 (2001), 123-128.

[119] X.H. Li and L.G. Wang, Monochromatic stars in rainbow $K_{3}$-free and $S_{3}^{+}$-free colorings, Discrete Math. 343 (2020), 112131.

[120] X.H. Li and L.G. Wang, Gallai-Ramsey numbers for rainbow $S_{3}^{+}$and monochromatic paths, Discuss. Math. Graph. Theory (2019), in press.

[121] X.H. Li, L.G. Wang and X.X. Liu, Complete graphs and complete bipartite graphs without rainbow path, Discrete Math. 342 (2019), 21162126.

[122] Y.C. Liu and Y.J. Chen, Gallai and $\ell$-uniform Ramsey numbers of complete bipartite graphs, Discrete Appl. Math. 301 (2021), 131-139.

[123] H. Liu, C. Magnant, A. Saito, I. Schiermeyer and Y.T. Shi, Gallai-Ramsey number for $K_{4}$, J. Graph Theory 94 (2020), 192-205.

[124] H. Liu, R. Morris and N. Prince, Highly connected multicoloured subgraphs of multicoloured graphs, Discrete Math. 308 (2008), 5096-5121.

[125] H. Liu, R. Morris and N. Prince, Highly connected monochromatic subgraphs of multicolored graphs, J. Graph Theory 61 (2009), 22-44.

[126] H. Liu and P.P. Pach, The number of multiplicative Sidon sets of integers, J. Combin. Theory Ser. A 165 (2019), 152-175.

[127] H. Liu and Y. Person, Highly connected coloured subgraphs via the regularity lemma, Discrete Math. 309 (2008), 6277-6287.

[128] H. Liu, O. Pikhurko and M. Sharifzadeh, Edges not in any monochromatic copy of a fixed graph, J. Combin. Theory Ser. B 135 (2019), 16-43. 
[129] H. Liu and M. Sharifzadeh, Groups with few maximal sum-free sets, $J$. Combin. Theory Ser. A 177 (2021), 105333.

[130] H. Liu, M. Sharifzadeh and K. Staden, On the maximum number of integer colourings with forbidden monochromatic sums, Electron. J. Combin. 28 (2021), Paper No. 1.59, 35 pp.

[131] B. Llano and A. Montejano, Rainbow-free colorings for $x+y=c z$ in $\mathbb{Z}_{p}$, Discrete Math. 312 (2012), 2566-2573.

[132] C. Lo, H.H. Wu and Q.Q. Xie, Monochromatic $k$-connected subgraphs in 2-edge-colored complete graphs, arXiv:2008.09001v2, (2020).

[133] J. Ma, On edges not in monochromatic copies of a fixed bipartite graph, J. Combin. Theory Ser. B 123 (2017), 240-248.

[134] W. Mader, Existenz $n$-fach zusammenhängender Teilgraphen in Graphen genügend grosser Kantendichte, Abh. Math. Sem. Univ. Hamburg 37 (1972), 86-97.

[135] F. Maffray and M. Preissmann, A translation of Tibor Gallai's paper: transitiv orientierbare Graphen, In: J.L. Ramirez-Alfonsin, B.A. Reed (Eds.), Perfect Graphs, Wiley, New York (2001), pp. 25-66.

[136] C. Magnant, A general lower bound on Gallai-Ramsey numbers for non-bipartite graphs, Theory Appl. Graphs 5 (2018), Art. 4, 6 pp.

[137] C. Magnant and P.S. Nowbandegani, Topics in Gallai-Ramsey Theory, Springer Briefs in Mathematics. Springer, Cham, Switzerland (2020).

[138] Y.P. Mao, Z. Wang, C. Magnant and I. Schiermeyer, Gallai-Ramsey numbers for fans, arXiv:1902.10706v1, (2019).

[139] Y.P. Mao, Z. Wang, C. Magnant and I. Schiermeyer, Ramsey and GallaiRamsey numbers for stars with extra independent edges, Discrete Appl. Math. 285 (2020), 153-172.

[140] D.W. Matula, Ramsey theory for graph connectivity, J. Graph Theory 7 (1983), 95-103. 
[141] K. Menger, Zur allgemeinen Kurventheorie, Fund. Math. 10 (1927), 96-115.

[142] S. Moran, M. Snir and U. Manber, Applications of Ramsey's theorem to decision tree complexity, J. Assoc. Comput. Mach. 32 (1985), 938-949.

[143] R. Morris and D. Saxton, The number of $C_{2 \ell}$-free graphs, Adv. Math. 298 (2016), 534-580.

[144] T.S. Motzkin, Cooperative classes of finite sets in one and more dimensions, J. Combinatorial Theory 3 (1967), 244-251.

[145] D. Mubayi, Edge-coloring cliques with three colors on all 4-cliques, Combinatorica 18 (1998), 293-296.

[146] D. Mubayi, An explicit construction for a Ramsey problem, Combinatorica 24 (2004), 313-324.

[147] D. Mubayi, Coloring triple systems with local conditions, J. Graph Theory 81 (2016), 307-311.

[148] D. Mubayi and A. Suk, A survey of hypergraph Ramsey problems, in: Discrete Mathematics and Applications, Springer, Cham, (2020), 405428.

[149] J. Nešetřil, Some nonstandard Ramsey-like applications, Theoret. Comput. Sci. 34 (1984), 3-15.

[150] J. Pach and I. Tomon, Colorings with only rainbow arithmetic progressions, Acta Math. Hungar. 161 (2020), 507-515.

[151] C. Pohoata and A. Sheffer, Local properties in colored graphs, distinct distances, and difference sets, Combinatorica 39 (2019), 705-714.

[152] S.P. Radziszowski, Small Ramsey numbers, Electron J. Combin. 1 (2021), Dynamic Survey 1, 116 pp.

[153] F.P. Ramsey, On a problem of formal logic, Proc. Lond. Math. Soc. (2) 30 (1930), 264-286. 
[154] F.S. Roberts, Applications of Ramsey theory, Discrete Appl. Math. 9 (1984), 251-261.

[155] V. Rosta, Note on Gy. Elekes's conjectures concerning unavoidable patterns in proper colorings, Electron. J. Combin. 7 (2000), Note 3, 3pp.

[156] V. Rosta, Ramsey theory applications, Electron. J. Combin. 11 (2004), Research Paper 89, 48 pp.

[157] A. Sah, Diagonal Ramsey via effective quasirandomness, arXiv:2005.09251v1, (2020).

[158] G.N. Sárközy and S. Selkow, On edge colorings with at least $q$ colors in every subset of $p$ vertices, Electron. J. Combin. 8 (2001), Research Paper 9, 6 pp.

[159] G.N. Sárközy and S. Selkow, An application of the regularity lemma in generalized Ramsey theory, J. Graph Theory 44 (2003), 39-49.

[160] D. Saxton and A. Thomason, Hypergraph containers, Invent. Math. 201 (2015), 925-992.

[161] I. Schur, Über die kongruenz $x^{m}+y^{m} \equiv z^{m}(\bmod p)$, Jahresbericht der Deutschen Mathematiker-Vereinigung 25 (1916), 114-117.

[162] A. Soifer (Editor), Ramsey theory: yesterday, today and tomorrow, Progress in Mathematics. Birkhäuser, Springer, New York (2011).

[163] Z.-X. Song, B. Wei, F.F. Zhang and Q.H. Zhao, A note on Gallai-Ramsey number of even wheels, Discrete Math. 343 (2020), 111725.

[164] Z.-X. Song and J.M. Zhang, A conjecture on Gallai-Ramsey numbers of even cycles and paths, Australas. J. Combin. 75 (2019), 296-308.

[165] J.H. Spencer, Ramsey's theorem-a new lower bound, J. Combinatorial Theory Ser. A 18 (1975), 108-115.

[166] J.H. Spencer, Asymptotic lower bounds for Ramsey functions, Discrete Math. 20 (1977), 69-76. 
[167] E. Szemerédi, On sets of integers containing no $k$ elements in arithmetic progression, Acta Arith. 27 (1975), 199-245.

[168] E. Szemerédi, Regular partitions of graphs, in: Problèmes Combinatoires et Théorie des Graphes, Colloques Internationaux CNRS Vol. 260, Orsay, 1976, pp. 399-401.

[169] B.L. van der Waerden, Beweis einer Baudetschen Vermutung, Nieuw Arch. Wisk. 15 (1927), 212-216.

[170] A.Z. Wagner, Large subgraphs in rainbow-triangle free colorings, $J$. Graph Theory 86 (2017), 141-148.

[171] Z. Wang, Y.P. Mao, H.Z. Li and S.P. Cui, Asymptotic lower bounds for Gallai-Ramsey functions and numbers, arXiv:2007.04895v1, (2020).

[172] Z. Wang, Y.P. Mao, C. Magnant, I. Schiermeyer and J.Y. Zou, GallaiRamsey numbers of odd cycles, arXiv:1808.09245v2, (2021).

[173] Z. Wang, Y.P. Mao, C. Magnant and J.Y. Zou, Ramsey and Gallai-Ramsey numbers for two classes of unicyclic graphs, Graphs Combin. 37 (2021), 337-354.

[174] Y. Wigderson, An improved lower bound on multicolor Ramsey numbers, Proc. Amer. Math. Soc. 149 (2021), 2371-2374.

[175] H.B. Wu, C. Magnant, P.S. Nowbandegani and S.M. Xia, All partitions have small parts-Gallai-Ramsey numbers of bipartite graphs, Discrete Appl. Math. 254 (2019), 196-203.

[176] A.C.C. Yao, Should tables be sorted? J. Assoc. Comput. Mach. 28 (1981), 615-628.

[177] J.M. Zhang, Two Ramsey-related problems, $P h D$ thesis, University of Central Florida (2019).

[178] F.F. Zhang, Z.-X. Song and Y.J. Chen, Multicolor Ramsey numbers of cycles in Gallai colorings, arXiv:1906.05263v1, (2019). 
[179] F.F. Zhang, X.T. Zhu and Y.J. Chen, Gallai-Ramsey numbers for multiple triangles, Discrete Appl. Math. 298 (2021), 103-109.

[180] Q.H. Zhao and B. Wei, Gallai-Ramsey numbers for graphs with chromatic number three, Discrete Appl. Math. 304 (2021), 110-118.

[181] J.Y. Zou, Y.P. Mao, C. Magnant, Z. Wang and C.F. Ye, Gallai-Ramsey numbers for books, Discrete Appl. Math. 268 (2019), 164-177. 


\section{Acknowledgements}

When I was sitting at the window and watching the weather changing between sunny and rainy, many memories came to my mind. In the past four years, I received a lot of help and support from many people. I would like to take this opportunity to express my sincere gratitude to them.

First of all, I would like to thank Professor Ligong Wang, my supervisor at Northwestern Polytechnical University (NPU). I have been studying with him when I was an undergraduate. He introduced me to the filed of graph theory and combinatorics when I attended the National College Students Innovation and Entrepreneurship Training Program. In the past few years, he gave me a lot of guidance, encouragement and positive supervision. He patiently taught me how to perform scientific research and write academic papers. He also encouraged me to discuss with other scholars and supported me to participate in many conferences and to visit the University of Twente (UT). I hereby would like to express my heartfelt thanks to my supervisor Professor Ligong Wang.

I would like to express my sincere gratitude to Professor Hajo Broersma, my supervisor at UT. I am very grateful to him for giving me the precious opportunity to study and perform research under his supervision. Every time I discussed problems with him, he was always very patient and energetic, and made valuable comments and suggestions. I also learned a lot of writing skills from his revision of my manuscripts. In the special time when we worked from home, he offered me generous help, support and encouragement. He also helped me a lot in applying for the CSC scholarship and handling graduation issues in UT. His kindness, wisdom and integrity will continue to influence my 
life.

My deep thanks also go to all my colleagues in the team of Graph Theory and Combinatorics at NPU, in particular Professor Shenggui Zhang who organized many conferences and lectures which gave me opportunities to learn about academic frontier and know other scholars.

I would also like to thank all my colleagues in the group of Formal Methods and Tools at UT. They gave me a great help both in life and work. I really enjoyed my time in the group.

My sincere thanks also go to all my friends I met in Enschede who gave me a lot of support and help.

My deepest thanks go to my grandparents and parents. Without their selfless love, I wouldn't have got where I am today.

Xihe Li

June 2021, Enschede 


\section{About the Author}

Xihe Li was born on October 10, 1995 in Ling County, Shandong Province, P.R. China. From 2001 to 2003, Xihe Li completed the first two years of elementary education in his village Lijiahutong. From 2003 to 2007, he completed his last four years of elementary education in Mei Town. After completing his junior middle school and senior middle school educations in Ling County, he started to study at the Department of Applied Mathematics (now School of Mathematics and Statistics), Northwestern Polytechnical University in September 2013.

After obtaining his Bachelor degree in June 2017, he was selected to become a PhD candidate directly under the supervision of Professor Ligong Wang at Northwestern Polytechnical University. Starting from October 2019, he visited the group of Formal Methods and Tools, University of Twente as a joint $\mathrm{PhD}$ student to perform research on Gallai-Ramsey theory under the supervision of Professor Hajo Broersma. The research has been sponsored by the China Scholarship Council. The main results obtained from his research during his PhD work have been collected in the current thesis. 



\section{$\mathrm{T}$}

his thesis contains a number of new contributions to the area in discrete mathematics that is commonly known as Gallai-Ramsey theory. We also contribute some new results related to this research area. In particular, we deal with the Erdős-Gyárfás function with respect to Gallai-colorings, and the rainbow Erdös-Rothschild problem with respect to 3-term arithmetic progressions.

One fundamental problem in the research of edge-colored graphs is to study the existence of nice substructures in an edge-colored host graph. In this thesis, the nice substructure we consider is either a rainbow subgraph or a monochromatic subgraph. The host graph we consider is a complete graph. 